\title{
Optical probes of free charge generation in organic photovoltaics
}

Alexander J. Barker

\author{
A thesis \\ submitted to Victoria University of Wellington \\ in fulfilment of the \\ requirements for the degree of \\ Doctor of Philosophy \\ in Physics.
}

Victoria University of Wellington

2015 



\begin{abstract}
Organic photovoltaics (OPVs) show considerable promise as a source of low cost solar energy. Improving our understanding of the processes governing free charge photogeneration in OPVs may unlock the improvements in efficiency required for their widespread implementation. In particular, how do photogenerated charge pairs overcome their mutual columbic attraction, and what governs the branching between bound and free charge pairs that is observed to occur shortly after their creation?

Ultrafast laser techniques such as transient absorption (TA) spectroscopy are the only tools capable of probing the time scales associated with these processes (as short as $10^{-14}$ seconds). Challenges include achieving sufficient sensitivity to resolve the tiny signals generated in thin films under solar-equivalent excitation densities, and distinguishing and quantifying overlapping signals due to separate phenomena.
\end{abstract}

We present the development of a versatile and ultra-sensitive broadband TA spectrometer, along with a comprehensive analysis of the noise sources limiting sensitivity. Through the use of referenced shot-to-shot detection and a novel method exploiting highly chirped broadband probe pulses, we are capable of resolving changes in differential transmission $<3 \times 10^{-6}$ over pump-probe delays of $10^{-13}-10^{-4}$ seconds.

By comparing the absorption due to photogenerated charges to measurements of open-circuit voltage decay in devices under transient excitation, we show that TA is able to quantify the recombination of freely extractable charge pairs over many decades of pump-probe delay. The dependence of this recombination on excitation density can reveal the relative fraction of bound and free charge pairs. We apply this technique to blends of varying efficiency and find that the measured free charge fraction is correlated with published photocharge yields for these materials.

We access a regime at low temperature where thermalized charge pairs are frozen out following the primary charge separation step and recombine monomolecularly via tunneling. The dependence of tunneling rate on distance enabled us to 
fit recombination dynamics to distributions of recombination rates. We identified populations of charge-transfer states and well-separated charge pairs, the yield of which is strongly correlated with the yield of free charges measured via their intensity dependent recombination. We conclude that populations of free charges are established via long-range charge separation within the thermalization timescale, thus invoking early branching between free and bound charges across an energetic barrier. Subject to assumed values of the electron tunneling attenuation constant, we find critical charge separation distances of $\sim 3-4 \mathrm{~nm}$ in all materials.

TA spectroscopy probes the absorption of excited states, with the signal being proportional to the product of population density and absorption cross-section of the absorbing species. We show that the dependence of signal on probe pulse intensity can decouple these parameters, and apply a numerical model to determine the time-dependent absorption cross-section of photogenerated excitons in thin films of semiconducting polymers.

Collectively, this thesis presents spectroscopic tools and applications thereof that illuminate the process of free charge generation in organic photovoltaics. 


\section{Acknowledgments}

After working outside of academia for three years, I was a little daunted by the decision to return and pursue a PhD. The first problem was to find a thesis supervisor; I'm pleased to say that the choice to work with Justin Hodgkiss is one of the best decisions I have ever made.

Beginning in February 2011, Kai Chen and myself had the honour of being Justin's first two PhD students. Three and some years later, I can't believe how much I have grown as a scientist, and how much fun it has been. Writing this is difficult as I'll never do it justice: Justin's incredible scientific insight, guidance, patience and genuine care for the people in his group has set a very high bar for my opinion of how a research group should operate. Thank you Justin! It was a blast.

Being such a young group, my fellow students and I relied heavily on each other while we developed the new spectroscopy lab. First and foremost lies the indomitable and kind Kai Chen, a true laser wizard with an encyclopedic knowledge of optics and unmatched ability to align the un-alignable (even if it results in a few laser burns to the new white walls). Big thanks also to Joe Gallaher and Shyamal Prasad for being fantastic coworkers and friends.

Thank you also to the stellar group of people that enriched my life in Wellington. The parade of flatmates and friends that passed through our beautiful house on Garden Road, my bandmates in The Blue Onesies, Walter Sommerville and the Cake Makers, the Chris Munroe Fan Club, the MESA committee, and the MacDiarmid Institute in general.

Finally, I am immensely appreciative of my wonderful parents and family, who have always supported me in every way possible. Fresh in my mind right now are the frozen packages of whitebait and other homegrown food that showed up at my door during the most intense days of writing this thesis. Incredible. I am a very lucky man and I love you all! 


\section{Contents}

1 Introducing the photophysics of organic photovoltaics 1

1.1 Conjugated polymers . . . . . . . . . . . . . . 2

1.2 Organic optoelectronic devices ............... 8

1.3 Improving OPV efficiency . . . . . . . . . . . . . . . . . . . . . . . . . . .

1.3.1 Polymer design . . . . . . . . . . . . . . . 14

1.3.2 Morphology . . . . . . . . . . . . . . 16

1.3.3 The puzzle and emerging theory of free charge photogeneration ...................... 17

1.4 Spectroscopy as a probe of charge photogeneration $\ldots . . \ldots 23$

2 Construction of an ultra-sensitive transient absorption spectrometer 27

2.1 Introduction . . . . . . . . . . . . . . . . . . 28

2.2 Experimental details . . . . . . . . . . . . . . . . 28

2.2.1 Excitation light sources and pulse timing . . . . . . . . . 29

2.2 .2 Probe light sources . . . . . . . . . . . . . . 30

2.2 .3 Light detection . . . . . . . . . . . . . . . 31

2.2 .4 System automation . . . . . . . . . . . . . 32

2.3 Case study: Charge generation in PTBT:PC ${ }_{61} \mathrm{BM} \ldots \ldots . \ldots 35$

3 Ultrasensitive spectroscopy via highly-chirped broadband probes 43

3.1 Introduction . . . . . . . . . . . . . . . . . . 43

3.2 Noise sources in transient absorption spectroscopy . . . . . . . . . . 44

3.2.1 Analysis ... . . . . . . . . . . . . . 44

3.3 Data analysis \& highly chirped broadband pulses for noise reduction 46

3.3.1 Chirp and effective sample number . . . . . . . . . . . . 48

3.3.2 Quantification of sensitivity enhancement . . . . . . . . 50

3.4 A recipe for instant, easy improvement in sensitivity . . . . . . . . . 55 
3.4.1 Limits of detection; optimization . . . . . . . . . . . . . 55

3.4.2 Reduction of IRF due to chirp . . . . . . . . . . . . . . . 57

3.5 Experimental details . . . . . . . . . . . . . . . . . . 58

3.6 Conclusions . . . . . . . . . . . . . . . . . 58

4 Charge recombination in polymer:fullerene blends 59

4.1 Introduction . . . . . . . . . . . . . . . . . . 59

4.2 Experimental: Sample preparation . . . . . . . . . . . . 61

4.3 Broadband TA spectra .................... 62

4.3 .1 P3HT ....................... 63

4.3 .2 PCDTBT ....................... 68

4.3 .3 PCPDTBT .................... 71

4.3 .4 MEH-PPV . . . . . . . . . . . . . 73

4.4 Correlation of recombination rate with photocurrent efficiency . . . 75

4.5 Fitted intensity-dependent TA . . . . . . . . . . . . . 76

4.6 Reconciliation of optical and electronic probes of recombination . . 82

4.7 Conclusions . . . . . . . . . . . . . . . . . . . 84

5 Distance distributions of photogenerated charge pairs in organic photo$\begin{array}{lr}\text { voltaic cells } & 85\end{array}$

5.1 Introduction . . . . . . . . . . . . . . . . 86

5.2 Experimental details . . . . . . . . . . . . . . . . . . 88

5.3 Results and discussion . . . . . . . . . . . . . . . . 88

5.4 Conclusions . . . . . . . . . . . . . . . . . . . . . 103

5.5 Appendix I: Early MEH-PPV:PCBM recombination . . . . . . . . . 104

5.6 Appendix II: Complete rate distribution fits . . . . . . . . . . . . 106

5.7 Appendix III: Polarization anisotropy . . . . . . . . . . . . . . . . 111

6 Ultrafast time-resolved measurement of absorption cross-section and population density via probe-saturation effects in TA spectroscopy 113

6.1 Introduction . . . . . . . . . . . . . . . . . . . 114

6.2 Qualitative interpretation ................. 115

6.2.1 Standard TA spectroscopy of F8BT . . . . . . . . . . . . . 116

6.2.2 Probe intensity-dependence . . . . . . . . . . . . . 118

6.3 A numerical model of TA probe absorption . . . . . . . . . . . . 120

6.3.1 Relevant transitions and rates . . . . . . . . . . . 120 
6.3.2 Implementation of the numerical model . . . . . . . . . . . 122

6.4 Results . . . . . . . . . . . . . . . . . 126

6.4.1 Two-photon absorption by excited states . . . . . . . . 130

6.4.2 Placing boundaries on sequential absorption effects . . . . . 132

6.5 Single-shot probe intensity dependence . . . . . . . . . . . . . . 134

6.6 Conclusions . . . . . . . . . . . . . . . . . . 136

$\begin{array}{lll}7 & \text { Summary \& future work } & 139\end{array}$ 


\section{Abbreviations}

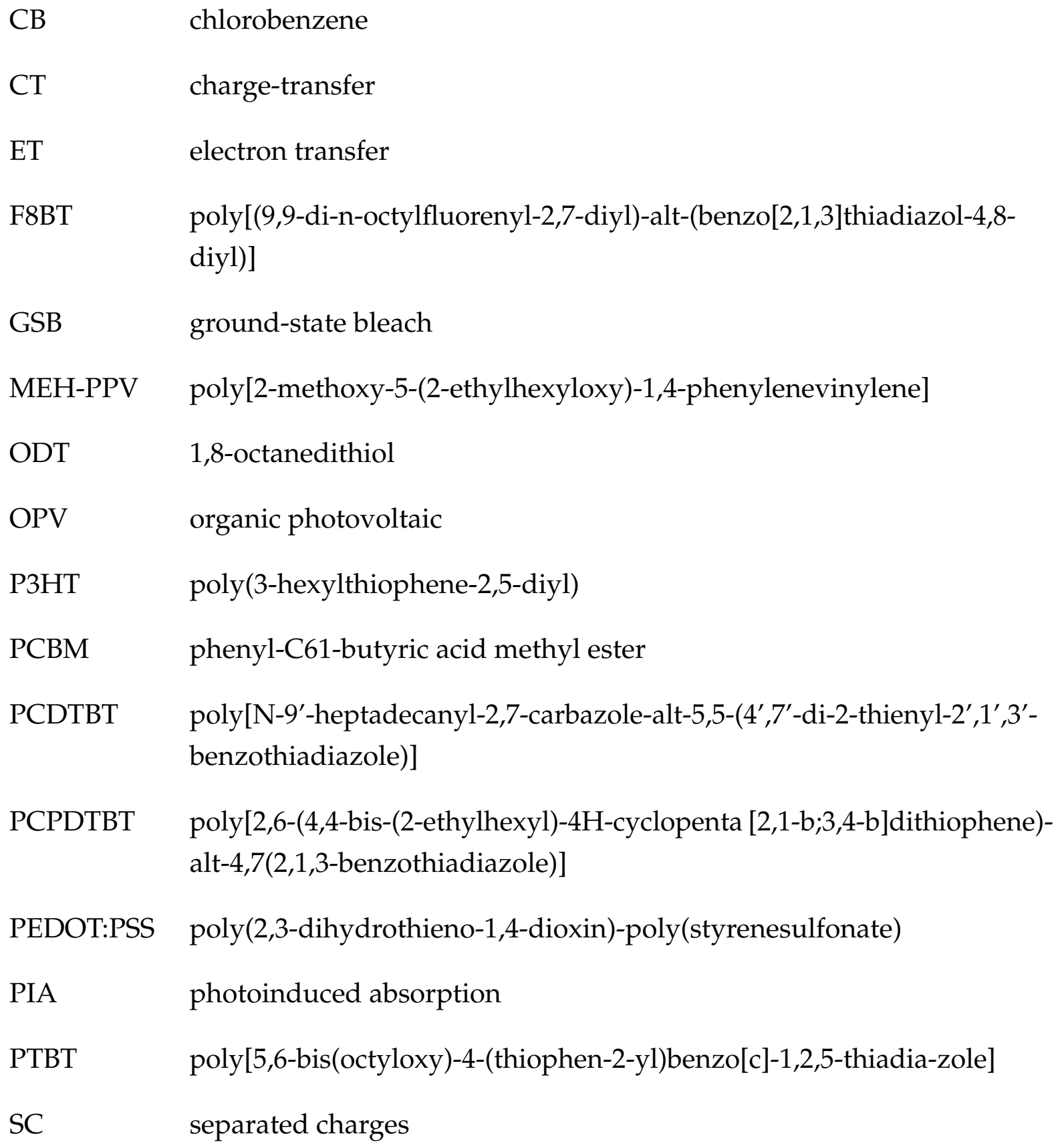


SE

stimulated emission

TA

transient absorption 


\section{Chapter 1}

\section{Introducing the photophysics of organic photovoltaics}

In a world where energy demand is growing at an unprecedented rate ${ }^{\mathrm{i}}$ and anthropogenic climate change is a present and scientifically proven threat, [2] few would deny that a global transition from reliance on fossil fuels (which account for around $70 \%$ of global greenhouse gas emissions [3]) is urgently required. The carbon-neutral energy solution that will enable such change is unlikely to come in the form of a single silver bullet; rather, many emerging technologies may develop to take advantage of energy generation niches, as determined by local environment, demand and economics. The available options for carbon-neutral energy fall in to three categories: sequestration of carbon from fossil fuel generation, nuclear power, and renewable (encompassing wind, solar, geothermal, hydroelectric and tidal power generation). That being said, when solely considering the absolute amount of available energy, solar energy dwarfs all other renewable and fossil-based resources combined. [4,5]

The photovoltaic (PV) industry has experienced enormous growth over the last five years, with total installed global PV capacity rising from 15.8 GW in 2008 to $139 \mathrm{GW}$ in 2013, [6] driven primarily by installations in Germany and (more recently) China. Of course, this still represents just a small fraction of the total global installed capacity from all energy sources of 5359 GW (2010 estimate, [7]). The vast majority of the currently installed PV capacity exhibits a solar power conversion efficiency of around 15\%, relying on an active layer of polycrystalline

\footnotetext{
${ }^{\text {i } T o t a l ~ g l o b a l ~ d e m a n d ~ w a s ~} 1.5 \times 10^{14} \mathrm{kWh}$ in 2010 , with a projected growth of $56 \%$ by 2040. [1]
} 
silicon, typically several hundred micrometres thick, manufactured at high tempurature in a clean room environment, and mounted in a heavy and rigid frame. While economies of scale and shared knowledge from the existing semiconductor industry has enabled significant reduction in the cost of polycrystalline silicon PVs, the required methods of manufacturing and installation present a limit to the affordability (and therefore the extent of implementation) of this technology.

Several emerging PV technologies exhibit promise for reducing the cost of solar energy by minmising the cost of manufacture and installation. [8] Examples include thin film chalcogenides such as cadmium telluride (CdTe) and copper indium gallium diseleneide (CIGS), quantum-dot and other nanostructured materials, dye-sensitised solar cells, $[9,10]$ the organo-metal halide perovskites, $[11,12]$ and small-molecule or polymer organic photovoltaics (OPVs).

Of these technologies, OPVs alone are able to be implemented as lightweight and flexible thin films, manufactured from Earth-abundant and non-toxic materials at room temperature, using scalable techniques such as solution-deposition and roll-to-roll processing. [13] The past decade has seen rapid advances in the performance of polymer OPVs, with reported power conversion efficiencies of $7.4 \%$ under solar illumination in peer-reviewed literature $[14,15]$, and up to $12 \%$ reported by industry for dual-layer OPVs. [16] Additionally, unlike silicon PVs, the performance of OPVs is not compromised with increasing operating temperature or at low light intensity. However, further improvements in power conversion efficiency and device longevity are required before widespread implementation can take place. The aim of this thesis is to develop new time-resolved spectroscopic tools and apply them to improve our understanding of the charge photogeneration process in OPVs, in order to guide the design of materials that meet these requirements.

\subsection{Conjugated polymers}

In $\pi$-conjugated polymers, overlapping

$\mathrm{p}_{z}$ orbitals form a chain of delocalized electron density along the backbone of the molecule. This conjugation is the main factor determining the electronic and optical properties of the molecule.

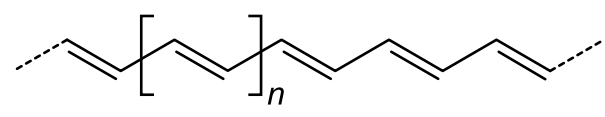

Figure 1.1: Polyacetylene, an archetypal conjugated polymer 
As more orbitals are added to the chain

(increasing the 'conjugation length'),

the spacing of energy levels decreases, resulting in band-like structure resembling that found in inorganic semiconductors. In this analogy, the highest occupied molecular orbital (HOMO) acts as the valence band, and the lowest unocupied molecular orbital (LUMO) as the conduction band. Unfortunately, the analogy does not hold up very far beyond this initial picture. In inorganic semiconductors, the presence of a free charge is readily screened by a readjustment of the surrounding charge density, on account of the high dielectric constant ( 12-13 [17]). Absorption of a photon therefore typically results in an electron-hole pair that are either completely dissociated, or weakly bound as a Wannier-Mott exciton with binding energy $\sim 10 \mathrm{meV}$ that readily dissociates at room temperature (where $\left.k_{\mathrm{B}} \mathrm{T} \sim 25 \mathrm{meV}\right)$ to form free carriers. [18, 19]

\section{Excitons}

Organic semiconductors typically exhibit a low dielectric constant ( 3-4 [17]), resulting in poor screening of charges. Along with strong electron-lattice coupling and weak intermolecular electronic coupling, this results in the primary excitations being tightly bound and comparatively localized Frenkel (or occasionally chargetransfer) excitons with typical binding energies ranging from $0.3-1.3 \mathrm{eV}$. [19-22] The energy of these excited states decreases with increasing conjugation length, in a manner that can be likened to the 'particle-in-a-box' model that will be (perhaps overly) familiar to undergraduate physics students and lecturers. This effect is clearly demonstrated in the shifting onset of optical absorption in a series of linear oligothiophenes in solution. [23] Indeed, the energy of the $S_{0} \rightarrow S_{1}$ transition was observed to decrease with increasing chain (and therefore conjugation) length (see Figure 1.2). The weaker dependence of the first excited triplet state $T_{1}$ energy on conjugation length is explained by the more localized nature of the triplet exciton with respect to the singlet.

\section{Molecular structure determines excited state behaviour}

It is clear that the extent of conjugation - and therefore the optical and electronic properties - of a molecule are intimately dependent on molecular structure. In a polymer, defects and structural deformations within the polymer chain can 


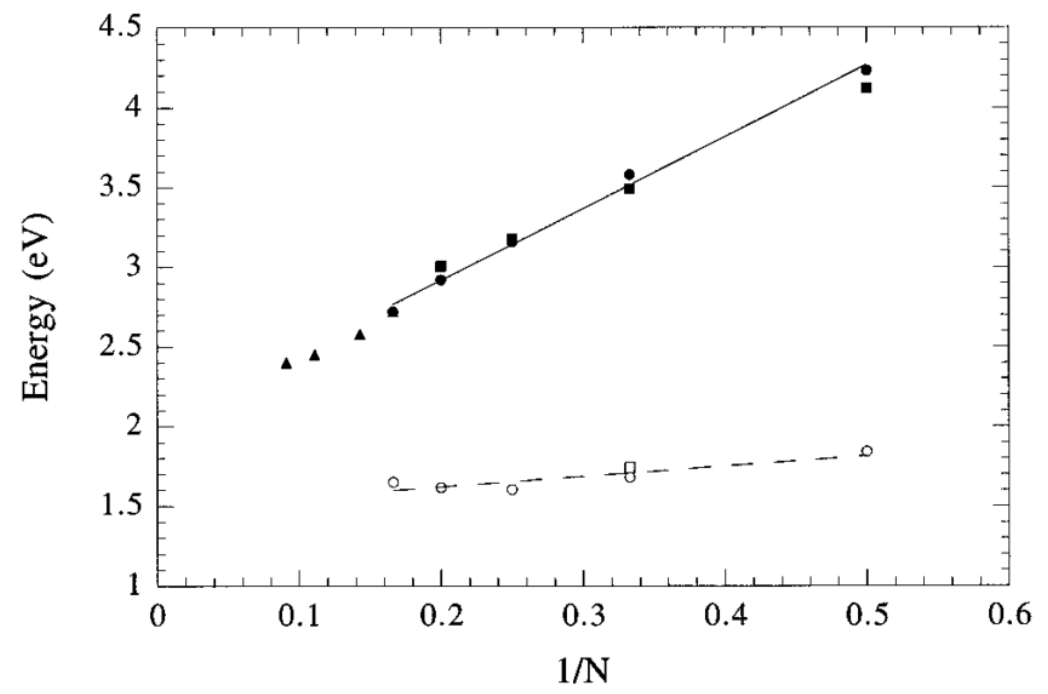

Figure 1.2: Measured dependence of $\mathrm{S}_{0} \rightarrow \mathrm{S}_{1}$ (closed symbols) and $\mathrm{S}_{0} \rightarrow \mathrm{T}_{1}$ (open symbols) energy on chain (and therefore conjugation) length for a series of linear oligothiophenes (figure reproduced from [23]).

misalign the $\mathrm{p}_{z}$ orbitals, breaking electron delocalization and forming isolated regions of conjugation that act as individual chromophores ( [24], see Figure 1.3). Variation in conjugation length between these conformational subunits gives rise to energetic dispersion of excited states throughout the material. Effects of this dispersion include inhomogeneous broadening of the fluorescence spectra, along with more salient repurcussions for OPVs that we will discuss shortly. It clearly follows that crucial electronic and optical properties including energy and delocalization of excited states, energetic dispersion, and electronic conductivity all depend on polymer chain conformation, and can be tuned as simply as through choice of solvent (in solution). These are truly molecular semiconductors. That being said, crystallization is important and (unsurprisingly) in the solid state additional effects come in to play. Chain organization and crystallization introduce long-range order and affect intermolecular electronic coupling, and can be altered as simply as heating a polymer film above its glass transition to allow the chains to reorganize. We will further discuss the role of morphology in the context of OPVs in Section 1.3.2.

Well ordered conjugated polymer films enable unbroken conjugation of orbitals over many repeat units. [25] However, while the singlet exciton exhibits delocalization by existing as a linear combination of several or many orbitals in an 


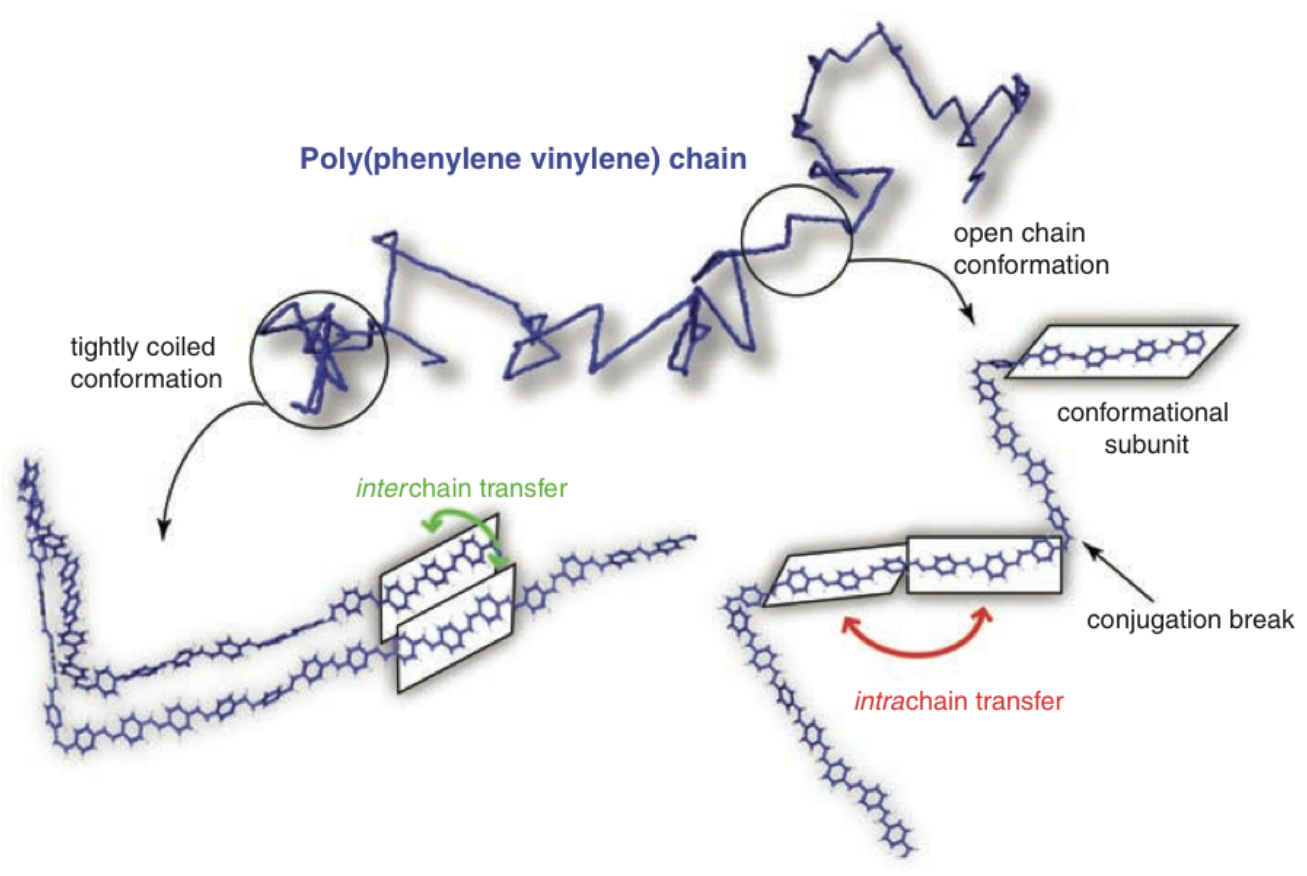

Figure 1.3: Conformational subunits illustrated for a polymer chain in solution (figure reproduced from [24]). Well ordered chains in polymer films can exhibit significantly longer conjugation lengths [25].

excited state, the extent of localization is not simply defined by the conjugation length of the polymer. The strong electron-vibrational coupling in conjugated polymers results in structural changes taking place in response to excitation. These deformations in combination with fluctuations from the environment can serve to dynamically localize the exciton, a process referred to as self-trapping. [26] The resulting changes in exciton size and excited state character take place over the tens of femtoseconds required for nuclear reorganization to occur, [22,27] and are reflected in the large Stokes shift characteristic of conjugated polymers. As we will see, this competition between delocalization due to overlapping orbitals and confinement due to electron-vibrational coupling is likely to play an important role in the charge generation process in OPVs.

\section{Charges/polarons}

Neutral excitons are not be the only excitation to be found in conjugated polymers. Indeed, if we are to generate electricity from sunlight we will need to consider free charges! Free charges can come to be in conjugated polymers either from 
exothermic dissociation of an exciton (resulting in charge transfer to an adjacent molecule), by chemical reduction/oxidation by a dopant molecule, or by injection/extraction of a charge from an outside source (such as at the interface of an electrode). [28] Similar to inorganic semiconductors, in conjugated polymers the presence of a free charge induces a geometric reorganization in the vicinity of the charge. The charge and accompanying lattice deformation are referred to as a polaron, a pseudo-particle with effective mass greater than that of the charge alone. As with excitons, the spatial extent of polarons in conjugated polymers is determined by competition between charge delocalization and structural-deformation induced confinement.

\section{Exciton and charge transport}

Transport of excitons and charges in conjugated polymers takes place via a number of incoherent mechanisms. [28] In general, the mechanism of transport may be short-range Dexter-type transfer relying on wavefunction overlap, or - in the case of excitons only - as long-range (Förster) resonant energy transfer, a nonradiative dipole-dipole interaction which depends on spectral overlap and dipole alignment of the energy donor and receiver. $[29,30]$ There have been indications that in crystalline polymer films intra- and inter-chain exciton transport are dominated by Dexter and Förster transfer respectively. [31] Both of these mechanisms can take place either by dispersive 'downhill' transport to lower energy sites within an inhomogeneously broadened density of states, or by slower thermally activated, diffuse transport (see Figure 1.4). [29,32,33] An interesting result of this is increasing charge mobility with increasing temperature, in constrast to inorganic semiconductors. Along with excited state lifetime, exciton mobility determines diffusion length, an important parameter in OPVs. Diffusion length can be measured by thickness-dependent quenching of photoluminescence in bilayer films, with reported values of $5-14 \mathrm{~nm}$ in conjugated polymers. [34]

While the above incoherent processes certainly describe the majority of transport mechanisms in conjugated polymers, recent work has suggested that coherent (wave-like) transport may be possible, and may play in important role in OPVs. [35] 


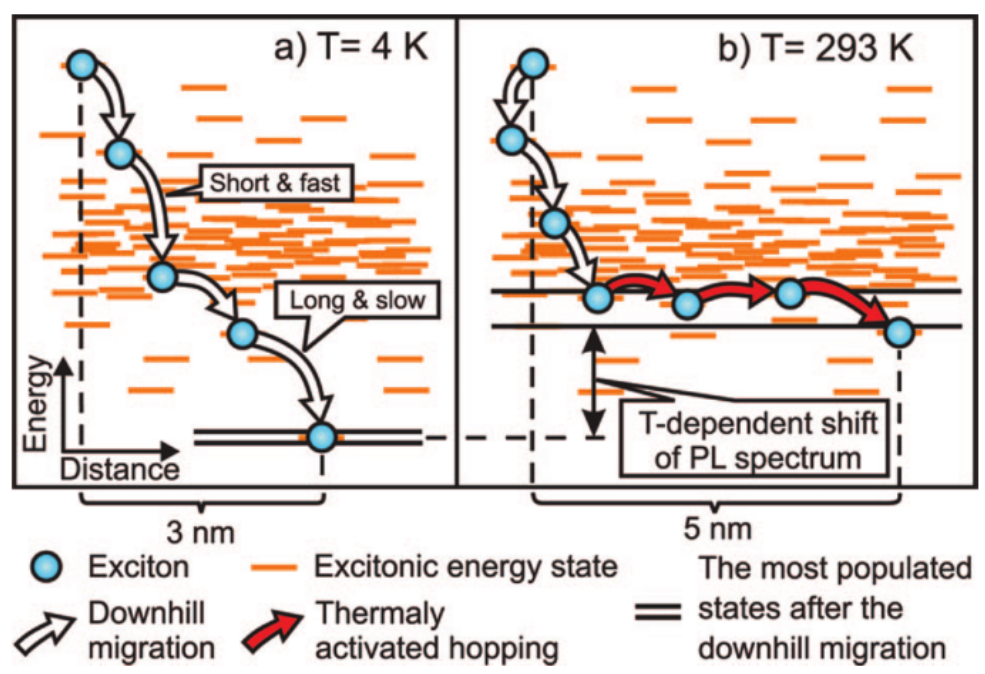

Figure 1.4: Exciton transport takes place by dispersive ('downhill' migration, fast and temperature independent), and by diffusive (slow, temperature dependent hopping) mechanisms (reproduced from [33]).

\section{Strong absorption and luminescence}

Absorption of photons to create a singlet exciton in the lowest excited state $S_{1}$ typically corresponds in conjugated polymers to a strongly dipole allowed $\pi-\pi^{*}$ transition. The materials therefore exhibit extremely high molar extinction coefficients (i.e. ground state absorption cross-section), high luminescence quantum yield (>60\% [36]), and stimulated emission, with $S_{1}$ excitons typically decaying within 0.1 to $1 \mathrm{~ns}$. [37] These characteristics, along with electronic conductivity and ease of processing, make conjugated polymers ideal as the active component in opto-electronic devices, as we will see in the following section.

\section{Triplet excitons}

In a singlet exciton, the excited electron (which can now be considered to occupy the lowest unoccupied molecular orbital (LUMO)) has spin antiparallel to that of the remaining electron in the highest occupied molecular orbital (HOMO). In configurations where the spin is not antiparallel, the state is referred to as a triplet exciton. In conjugated polymers, the direct creation of triplet excitons from the ground state by absorption of a photon is a dipole-forbidden transition, and therefore takes place at a negligible rate. [17] Additionally, the weak spinorbit couping in the light atomic consituents of conjugated polymers results in 
a very slow rate of intersystem crossing between singlet and triplet states. Accordingly, relaxation of triplets direcly to the ground state is a very slow process, and creation of triplets generally represents a loss mechanism in OPVs. Triplet excitons in conjugated polymers can, however be efficiently generated by fission of a singlet excitation, [38,39] recombination of uncorrelated charges (such as in a light-emitting diode), [40] [41] or by intersystem crossing facilitated by heavy metal dopants. [42]

\subsection{Organic optoelectronic devices}

The optical and electronic properties of conjugated polymers discussed thus far (strongly absorbing and luminescent in the visible spectrum, electronically conductive, with tunable energetics) along with the abundance of their raw materials and low cost and ease of fabrication makes them ideally suited for use in a range of optoelectronic devices. In this section we review the work to date towards the development of practical organic polymer optoelectronics.

\section{Organic transistors and light emitting diodes}

Small molecule organic light emitting diodes (OLEDs) were first demonstrated in 1987 [43] but faced issues with reliability. The first successes with conjugated polymer devices came with the invention by Burroughes and coworkers of the polymer OLED in 1990, [44,45] where charges injected into highly crystalline PPV recombine to form luminescent excitons. Advantages over small molecule OLEDs include ease of fabrication and improved long-term stability.

Field-effect transistors (FETs) and diodes have been constructed as early as 1988 using conjugated polymers as a p-type active layer. [46] Polymer FETs were used to observe strong dependence of charge mobility on polymer crystal orientation, indicating interchain delocalization of charges and accompanying two-dimensional charge transport due to the strong intermolecular $\pi-\pi$ interactions in P3HT. [47] Polymer FETs have even been made by inkjet printing. [48] More recently, n-type polymer FETs have been demonstrated, [49] which may enable the construction of organic complementary metal-oxide semiconductor (CMOS) circuits. 


\section{Conjugated polymer lasers}

The multilevel excited state nature of conjugated polymers in conjungtion with their ease of fabrication, flexibility, strong absorption, luminescence, and stimulated emission, makes them excellent candidates as the active material in diode lasers. In $1992 \mathrm{MEH}-\mathrm{PPV}$ was shown to lase when optically pumped in solution, with an efficiency comparible to the commonly used dye Rhodamine 6G. [50] Intermolecular effects were initially found to quench stimulated emission in neat MEH-PPV films, preventing application as a laser diode gain medium. Developments in understanding and controlling film morphology and purity proved to be essential, with Graupner and coworkers using ultrafast spectroscopy to detect stimulated emission in well ordered neat films of ladder-type polymers. [51] Further developments included the use of two-polymer blends, where energy transfer from the 'host' polymer to the 'guest' served to shift the emission wavelength to a region of lower absorption, reducing losses and lowering the threshold of amplified stimulated emission. [52] Since then, optically pumped polymer lasers have been constructed using a range of resonant structures. However, the goal of a polymer diode laser has not yet been achieved, with charge induced absorption generally cited as the main barrier to their operation. [36]

\section{Organic photovoltaics}

Early efforts to construct photovoltaic cells using pure conjugated polymers in a Schottky diode structure resulted in power conversion efficiencies on the order of $0.01 \%$, [53], where the difference in work function of the electrodes proved insufficient to overcome the tight exciton binding. A breakthrough came with the discovery of photoinduced charge transfer from the polymer MEH-PPV to an electron acceptor, buckminsterfullerene $\left(\mathrm{C}_{60}\right)$. [54] The highly effective quenching of polymer photoluminescence by $\mathrm{C}_{60}$ indicated that charge transfer was an extremely fast process, separating the bound excitons at a rate competing with that of exciton decay.

The strongly visible absorption of conjugated polymers enables thin PV active layers, on the order of $100 \mathrm{~nm}$ (some three orders of magnitude less than that for crystalline solar cells. [55]). However, the addition of a $\mathrm{C}_{60}$ electron accepting layer to form a planar heterojunction is not enough to create an efficient photovoltaic device, as the majority of absorbed photons create excitons in the polymer bulk 


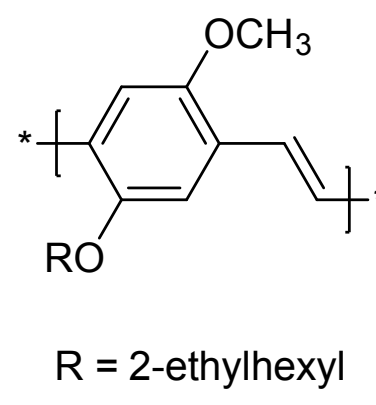

(a) MEH-PPV (donor)

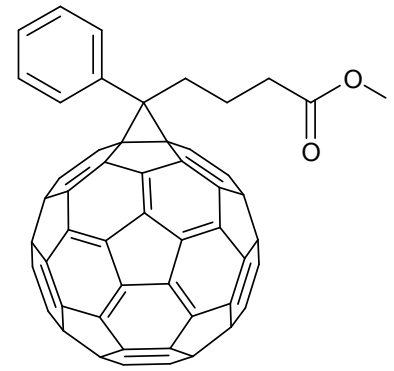

(b) PCBM (acceptor)

Figure 1.5: Components of the first bulk-heterojunction OPVs.

that decay before diffusing to the polymer/fullerene interface (see Figure 1.6a). [37]

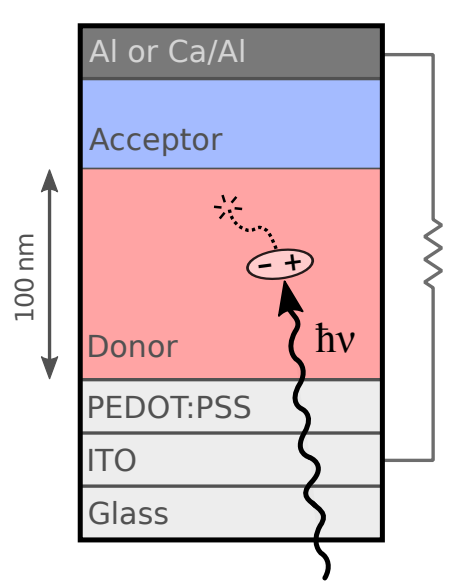

(a) Planar device structure

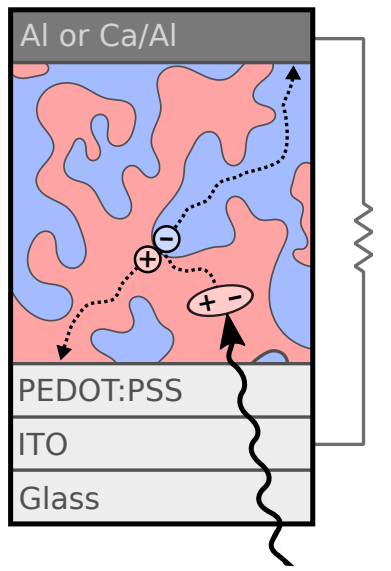

(b) Bulk heterojunction structure

Figure 1.6: The most commonly used OPV device structures employ a transparent indium tin oxide (ITO) anode, PEDOT:PSS hole transport layer, and aluminium or calcium/aluminium cathode. The use of a bulk heterojunction enables efficient collection of excitons, which would typically decay before reaching the interface in a planar device.

\section{Bulk heterojunction OPVs}

The emergence of the modern OPV structure came about with the introduction of the bulk heterojunction simultaneously introduced in an all polymer blend by Halls et al. [56] and in a polymer:fullerene blend by Yu et al. [57], where the soluble fullerene derivative PCBM was blended with the polymer before being spin-cast 
as a film. The electron acceptor (PCBM) and donor (the polymer MEH-PPV) were found to phase separate into an interpenetrating bicontinuous network, reducing the average distance of the donor-acceptor interface from any point in the bulk, while allowing optimized device thickness and maintaining a charge extraction pathway to the electrodes (Figure 1.6b). The resulting power conversion effiency of $2.9 \%$ was two orders of magnitude greater than that of the neat polymer devices, establishing the polymer:fullerene bulk heterojunction illustrated in Figure $1.6 \mathrm{~b}$ as the standard architecture on which the vast majority of subsequent OPV research has been based.

The basic picture of charge photogeneration in a donor-acceptor blend is outlined in Figure 1.7. In this model, $[37,58]$ absorption of a photon in the donor generates a bound electron-hole pair (1), which relaxes, undergoing energeticallydriven transport to lower energy sites in the the polymer (2). The exciton may diffuse to the interface between the donor and the acceptor (3) where the difference $\mathrm{E}_{\mathrm{D}, \mathrm{LUMO}}-\mathrm{E}_{\mathrm{A}, \mathrm{LUMO}}$ drives the transfer of an electron onto the acceptor. Following charge transfer (CT), the charge pair may be sufficiently separated to overcome their mutual Coulombic attraction and fully dissociate, enabling transport (5) to their respective electrodes, where they can be extracted as useful photocurrent.

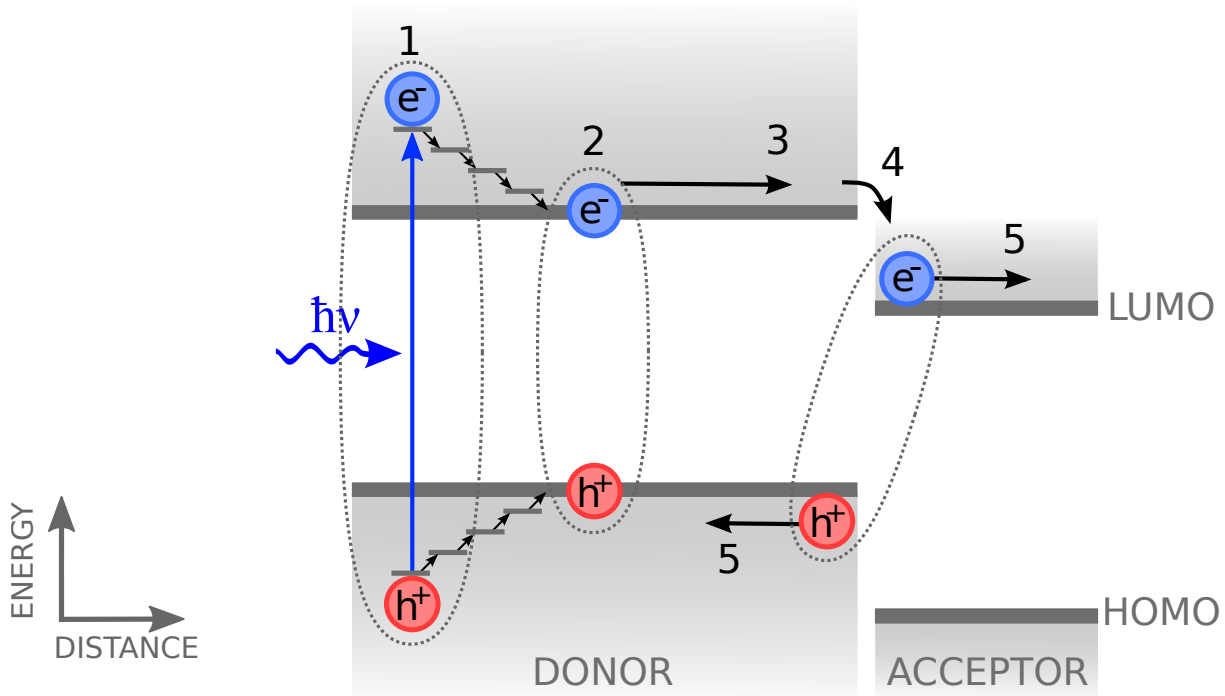

Figure 1.7: Excitation (1), relaxation (2), diffusion to the interface (3), charge transfer (4), separation of the electron and hole (5). 


\subsection{Improving OPV efficiency}

\section{What determines efficiency?}

The power conversion efficiency, $\eta$, of a solar cell is determined by its ability to collect photons across the solar spectrum, convert them into charges, and deliver those charges to the electrodes with a minimum loss of charge number (photocurrent) and potential energy per charge (photovoltage). $\eta$ is defined as the ratio of output power to incident power on the cell when operating at the maximum power point (i.e. with an optimized load). The output power at the maximum power point is given by $P_{m}=V_{\mathrm{OC}} \times J_{\mathrm{SC}} \times \mathrm{FF}$, where $V_{\mathrm{OC}}$ is open-circuit voltage, $J_{\mathrm{SC}}$ is short-circuit current density, and FF is fill-factor. [59]

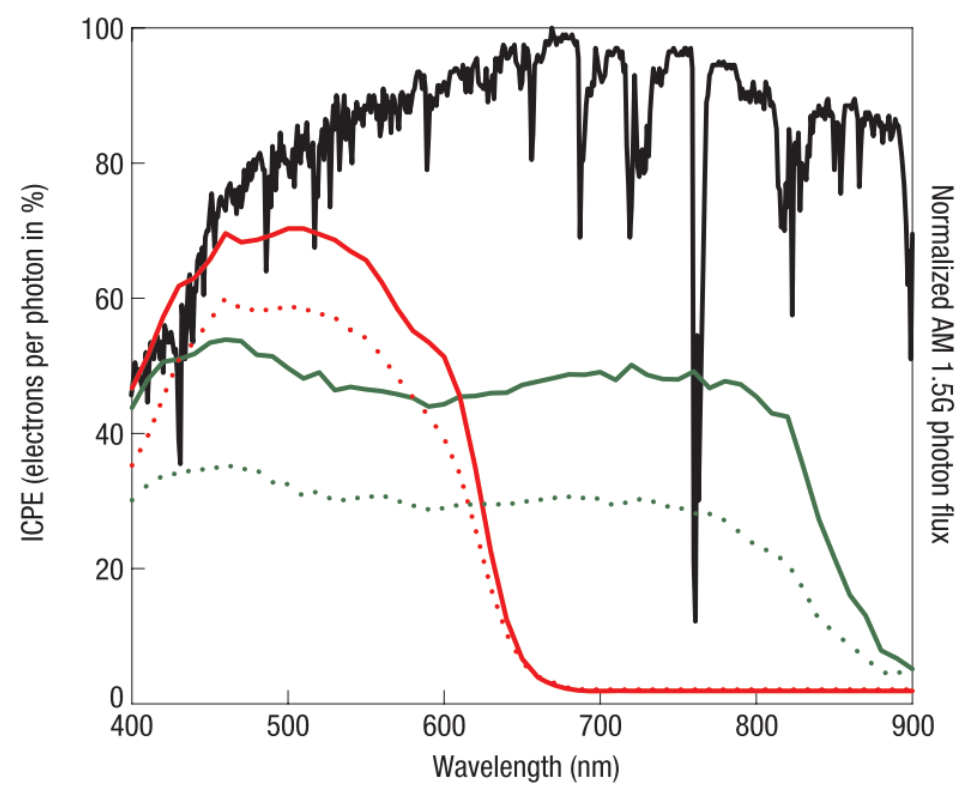

Figure 1.8: External quantum efficiency of P3HT:PC ${ }_{61} \mathrm{BM}$ (red) and PCPDTBT:PC ${ }_{71} B M$ (blue) devices under solar illumination. The improvement from dotted to solid line is due to thermal annealing in the case of P3HT:PC ${ }_{61} \mathrm{BM}$, and to the addition of the cosolvent ODT in preparation of the PCPDTBT:PC ${ }_{71} \mathrm{BM}$ film, highlighting the importance of morphology in device performance. The black line shows the standard AM 1.5G solar spectrum, with structure due to atmospheric absorption (Figure reproduced from [60]).

The external quantum efficiency (EQE) is defined as the wavelength dependent ratio of the number of photogenerated charges collected at the electrodes to the number of photons incident on the cell. Integrating the product of EQE and the 
incident solar irradiance with respect to wavelength gives us the short-circuit current density $J_{\mathrm{SC}}$. Figure 1.8 shows EQE (presented as a percentage rather than a fraction) for two polymer:fullerene blends, with poor and optimized morphologies (dotted and solid lines, respectively). It is clear that the devices with optimized morphologies are significantly better at turning incident photons into useful charges at the electrodes. What is not clear is whether this enhancement is due to improvements in photon absorption, dissociation of excitons, separation of charges or transport of charges to the electrodes. We will see in Chapter 4 how ultrafast spectroscopic techniques can be used to determine the origin of these improvements.

$V_{\mathrm{OC}}$ is primarily determined by the difference in energy between the HOMO level of the donor and the LUMO level of the acceptor. [61] Reducing the LUMOLUMO offset between donor and acceptor increases $V_{\mathrm{OC}}$, at the cost of reducing the driving energy for charge transfer and, by extension, the charge yield $J_{\mathrm{SC}}$. A regularly used empirical expression for $V_{\mathrm{OC}}$ in polymer:fullerene OPVs is given by $V_{\mathrm{OC}}=(1 / e)\left(E_{\mathrm{HOMO}}^{\mathrm{D}}-E_{\mathrm{LUMO}}^{\mathrm{A}}-0.3 \mathrm{eV}\right)$, where $e$ is the elementary charge and $0.3 \mathrm{eV}$ is an experimentally determined value representing typical losses in a device. [62]

The fill factor FF represents the reduction in voltage and current density from the open-circuit and short-circuit values when operating at the maximum power point. In OPVs, low FF is often (but not exclusively) attributed to inefficiencies in charge extraction due to recombination, poor charge mobility (especially of the minority carriers) and poor interfacial morphology between the active layer and electrodes. [63]

Excitations generated by photons with energy greater than the band gap relax prior to charge extraction, and the excess energy is lost as heat. The optimum band gap is therefore determined by competition between photon collection and internal efficiency. In a single junction solar cell under AM 1.5 illumination (see Figure 1.8) the theoretical limit of efficiency is $33.7 \%$ for a cell with the optimum band gap of $1.34 \mathrm{eV}$ (corresponding to a wavelength of $925 \mathrm{~nm}$ ), as described by Shockley and Queisser in 1962. [64] Several approaches to exceeding this limit have been proposed, including designs that employ exciton fission [65] or photon upconversion [66] to reclaim excess energy or harvest sub-bandgap photons, repspectively. 


\subsubsection{Polymer design}

The number of polymers available for OPVs has grown rapidly in recent years, with an increasing number of companies providing materials as demand from research groups grows and commerical manufacture of OPVs draws closer. A representative range of polymers of varying performance (which happen to be materials studied in this thesis) are shown in Figure 1.9.

As the electronic and optical properties of these materials are determined by the conjugated orbitals along the polymer backbone, chemists can modify sidechains to affect mechanical properties with minimal effect on parameters such as band gap or intrachain mobility. Much of polymer design has therefore been to optimise the solid state morphology and, in particular, their interaction with fullerenes.
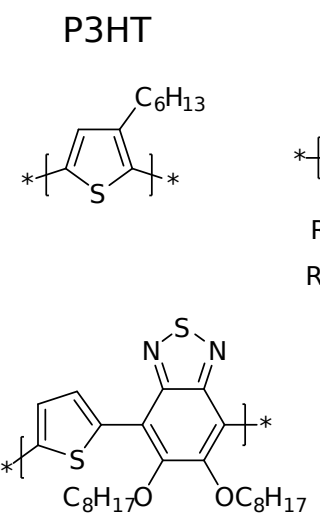

PTBT
MEH-PPV

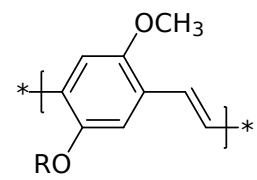

$\mathrm{R}=2$-ethylhexyl
PCPDTBT
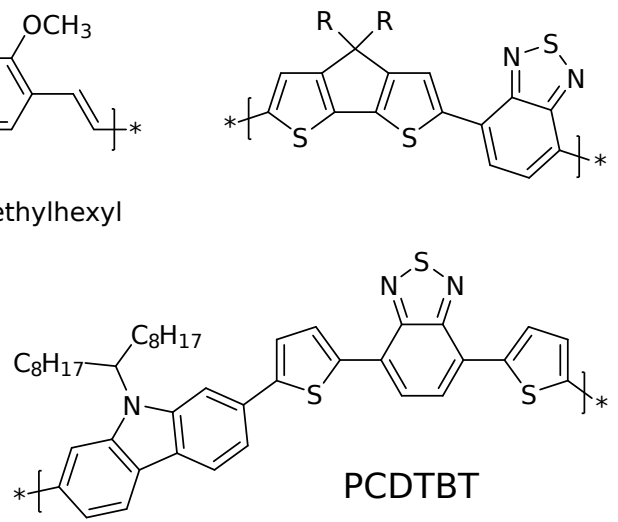

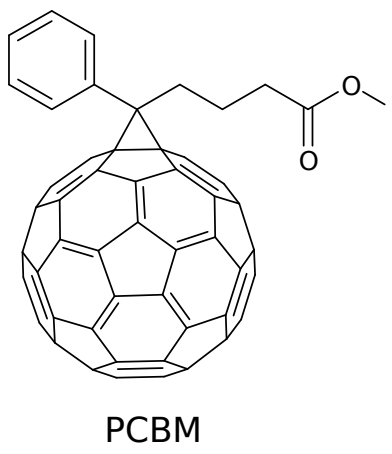

PCBM

Figure 1.9: Molecular structures of some of the $\pi$-conjugated polymers studied in this thesis, along with the electron acceptor PCBM.

The main progression in polymer design has been the push towards lower band gap polymers with broad and strong absorption further into the red of the solar spectrum. For instance, in order of development: the onset of absorption (in films) is around $560 \mathrm{~nm}$ for MEH-PPV, $630 \mathrm{~nm}$ for P3HT, $690 \mathrm{~nm}$ in PTBT and $830 \mathrm{~nm}$ in PCPDTBT, close to the optimum as determined by Shockley and Queisser. [64] State of the art polymers such as PCPDTBT, PCDTBT and PTB7 (not shown) tend to employ alternating copolymers with intrachain donor-acceptor character that serve to achieve a small band gap with low HOMO levels, thereby retaining sufficient LUMO-LUMO offset to drive efficient charge transfer. [67-69].

Advances in polymer design are continuing, but it is not clear what is required to unlock the full potential of these materials. Until the processes governing charge 
generation are well understood, the development of new materials will continue to rely on empirical rules of thumb and laborious trial and error. 


\subsubsection{Morphology}

Before returning to the charge photogeneration, we will briefly consider the role played by blend morphology in OPVs. The role of morphology broadly falls in to two categories: (1) ensuring well ordered crystalline structure that maximizes exciton and charge mobility and avoids defects that may act as recombination centers; and (2) engineering a large donor-acceptor interfacial area while ensuring bicontinuous structure to ensure pathways are available by which to transport free charges to the electrodes.

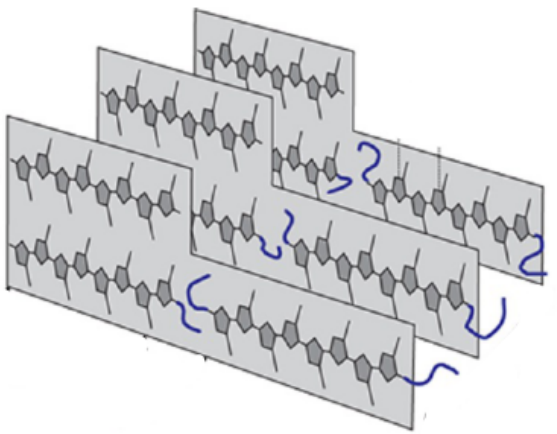

Figure 1.10: Semicrystalline structure of regio-regular P3HT (reproduced from [70]).

With exciton diffusion lengths of $5-14 \mathrm{~nm}$ in conjugated polymers, [34] it follows that polymer domains should be on the order of $10 \mathrm{~nm}$ to ensure excitons are likely to reach the interface. It turns out characterization of domain size is not a simple task: the amorphous nature of many OPVs hampers the use of X-ray diffraction, atomic-force microscopy is limited to probing the surface, and the low atomic numbers of the constituent elements does not allow for sufficient contrast between domains when using electron microscopy. [71] Optimization has therefore been largely a trial-and-error procedure.
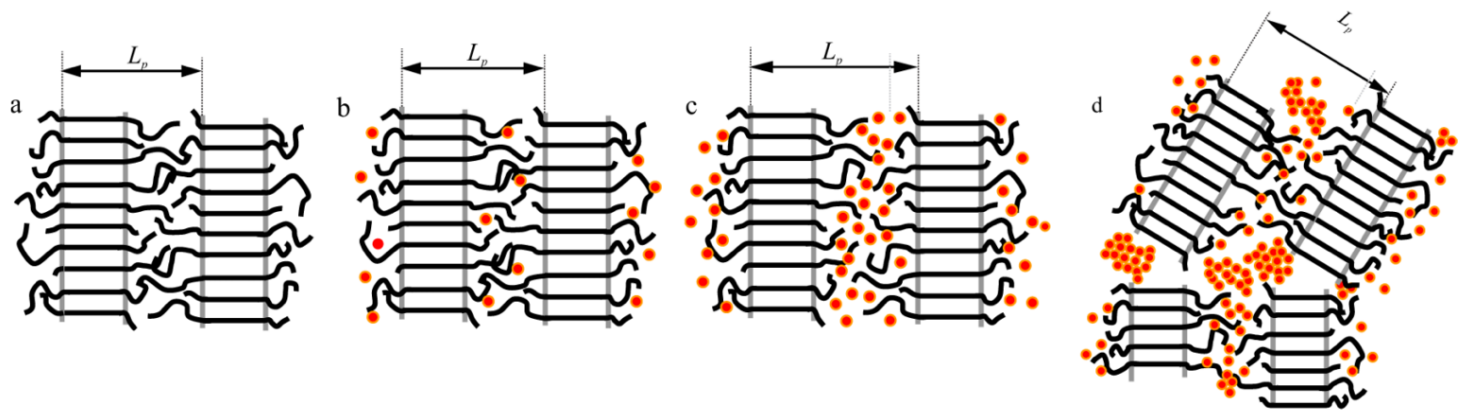

Figure 1.11: The evolution of domain formation in P3HT:PCBM with increasing PCBM fraction (reproduced from [70]).

Control of morphology for a given spin-coated donor/acceptor blend can be achieved though the choice of solvent(s), spin-coating rate, and by thermal or vapour annealing. Adjustment of the first two parameters primarily serves to vary 
the amount of time for the solvant to evaporate. Extremely rapid evaporation results in casting of an intimately mixed amorphous glass, while slowing the process allows the polymer to reorganize and crystallize, phase-separating from the PCBM, which is ejected from the polymer and forms domains (see Figure 1.11). [70-72] The same process is reinitiated after casting by thermal or vapour annealing, and in all cases, the growth of domains was accompanied by an improvement in device performance. [72] Indeed, this favourable energetic interplay between polymer and fullerene is likely to be a major factor in the longevity of PCBM as the acceptor of choice over nearly 20 years of OPV development.

The energetics of the interface itself are also affected by annealing, with electroluminescence spectroscopy and Fourier-transform photocurrent spectroscopy indicating a reduction of the energy of interfacial CT states upon annealing. [73,74]

In addition to controlling the donor-acceptor interface, slowing the drying process or annealing allows the polymer to reorganize into a more ordered, crystalline structure. The effects of regioregularity are also important, with highly regioregular samples ensuring extended and defect free crystal packing, and by extension exciton and charge mobility. [75] An additional effect of polymer regioregularity is to increase fullerene crystallization and domain formation, as the ordered polymer more effectively ejects fullerene molecules. [76] The resulting increase in mean conjugation length along the polymer backbone reduces the energy of the lowest excited state, red-shifting the absorption and emission spectra. Additionally, the reduction in energetic dispersion and increase in intermolecular electronic coupling affect charge transport properties and increase the vibronic structure apparent in the polymer absorption spectrum, $[77,78]$ suggesting the use of spectroscopy as a probe of morphology.

\subsubsection{The puzzle and emerging theory of free charge photogen- eration}

\section{Charge-transfer states}

The low dielectric constant and resulting poor electronic screening in conjugated polymers enables charges to Coloumbically interact over significant distances. As such, electron-hole pairs that have undergone charge transfer at the donor-acceptor interface (step (4) in Figure 1.7) may form bound interfacial states, referred to as charge-transfer (CT) states. The existence of CT states, the processes by which 
they dissociate and their role in the charge generation process are central to understanding charge photogeneration in OPVs and have accordingly been the subject of extensive research.

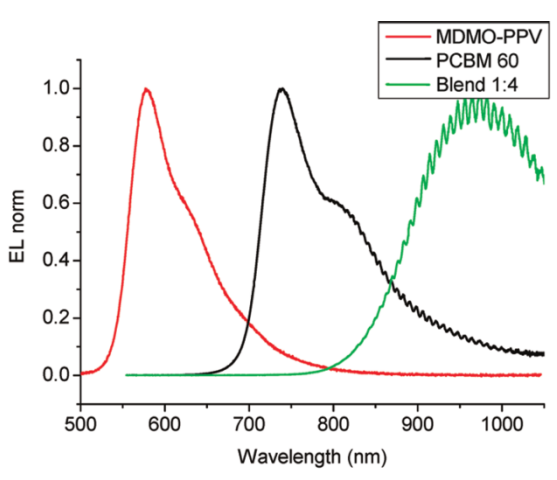

(a)
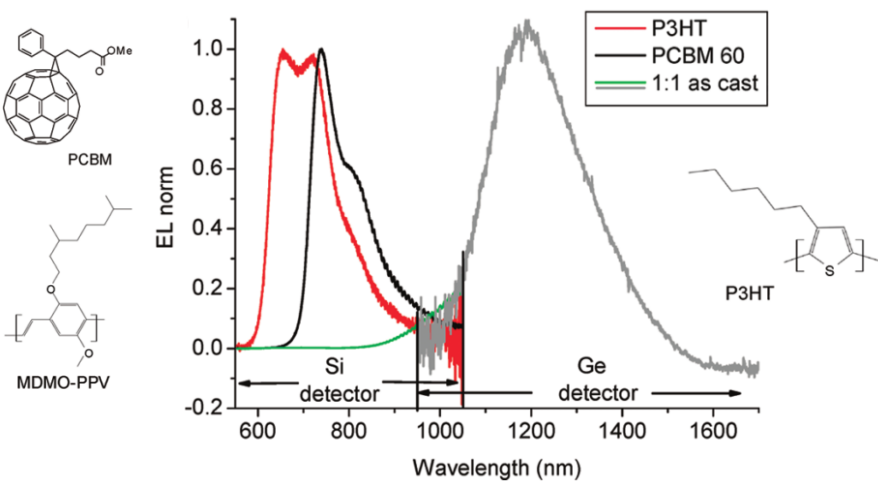

(b)

Figure 1.12: Electroluminescence in polymer:fullerene blends not present in either of the neat materials is associated to direct radiative recombination from a CT state (reproduced from [73]).

The weak electronic coupling of CT states with the ground state makes their detection and investigation particularly challenging. Groups have reported weak photoluminescence features in polymer:polymer OPV blends (such as F8BT:PFB) that were absent in either of the neat components. [79-81] The weak, redshifted, broad and long lived 'exciplex' emission provided good evidence for the existence of interfacial CT states. Similar luminescence was eventually detected in polymer:fullerene blends $[82,83]$. Electroluminescence spectroscopy revealed the existence of CT states in the well known blends MDMO-PPV:PCBM and P3HT:PCBM by monitoring luminescence due to the recombination of directly injected charges (see Figure 1.12). [73]

More recently, CT absorption transitions from the ground state have been identified [84]. While weak, these red-shifted absorptions provide an extremely useful tool for identifying the rates of processes occurring after electron transfer: since CT states are generated directly at the interface, any time-resolved behaviour is not blurred by the distribution of arrival times of excitons, as occurs when exciting above the band gap of materials throughout the bulk. 


\section{Initial models of charge photogeneration: The charge-transfer state as an inter- mediary}

The process by which photogenerated electron-hole pairs fail to escape their mutual attraction and ultimately recombine is referred to as geminate recombination, and was first formalized by Onsager in 1938. [85] In this model, photoexcited electrons initially undergo rapid thermal motion due to the excess energy of excitation, thermalizing at a distance from the localized hole. Onsager defines the Coulomb radius $r_{\mathrm{c}}$ as the intercharge distance at which Coulombic attraction equals the thermal energy, with $r_{\mathrm{c}}=e^{2} / 4 \pi \epsilon_{r} \epsilon_{0} k_{\mathrm{B}} T$. If the thermalized distance $a$ exceeds the Coulomb radius, the charges are dissociated. However if thermalization takes place within the Coulomb radius, the pair form a CT state with a probability of recombination that is dependent on applied electric field. The probability $P(E)$ at low field strengths is given by

$$
P(E)=\exp \left(\frac{-r_{\mathrm{c}}}{a}\right)\left(1+\frac{e r_{\mathrm{c}}}{2 k_{\mathrm{B}} T} E\right) .
$$

The Onsager model has formed the basis for the majority of models describing the dissociation of CT states in organic materials, with notable modifications to account for the finite $\mathrm{CT}$ state lifetime even at zero electron-hole separation [86] and to correct for the long mean free paths of charges in high mobility materials. [87] The model has been successfully applied to explain the electric field dependence of charge generation in neat polymers [88] and polymer:fullerene OPV devices. [89]

$\mathrm{CT}$ state binding energies of at least $250 \mathrm{meV}$ have been observed in polymer semiconductors, by comparing the emission energy of the CT state with that of the intrachain excitons [90]. It is this attraction that must be overcome in order for charge pairs to be fully separated. The extremely fast rate of exciton thermalization suggests that the amount of excess energy possessed by an electron after charge transfer should be defined by the LUMO-LUMO offset at the donor-acceptor interface. Measurements by Ohkita and coworkers on a series of polythiophenes with varying ionization potentials found a strong correlation between free energy difference for charge separation and charge polaron yield. [91]

Measurements in blends with low LUMO-LUMO offset found a dependence of charge photocurrent on exciton energy by varying excitation wavelength. [92] As this effect was not apparent in blends with significant LUMO-LUMO offset, it suggests that when sufficient driving energy is not built into the energetics of 
charge transfer at the interface, excess energy from hot excitons becomes important for charge separation.

Experiments looking to more direcly probe the role of CT states have been undertaken by measuring the $\mathrm{THz}$ polaron absorption [93] or charge photocurrent $[94,95]$ resulting from direct excitation of the weak sub-band gap CT absorption exhibited in some blends. By finding little loss in charge yield from these relaxed CT states when compared to those generated with excess energy from excitonic precursors, they question the role of excess energy in the dissociation of CT states. It is worth mentioning that in order to quantitatively compare charge yields from above- and below-band gap excitation requires renormalising data of significantly different magnitudes by measuring the sub-band gap absorption, which may be an appreciable source of error.

\section{Beyond Onsager: Delocalization and branching}

Limitations to the Onsager model become apparent with measurements failing to explain the extremely fast generation of charges following photoexcitation. Ultrafast spectroscopy has revealed that quenching of excitons into charges can take place in less than $100 \mathrm{fs}$, [96] more than an order of magnitude faster than the time required for transport of excitons over the length scales of polymer domains $(\sim 10 \mathrm{~nm})$ based on measured diffusion coefficients. [97]

The emerging model of charge generation invokes delocalization and/or enhanced mobility of excitations on ultrafast timescales that are able to couple to the manifold of higher lying charge transfer states, achieving the neccessary separation to overcome the Coulombic binding energy. $[35,67,96,98,99]$ We will now discuss some key results that have been instrumental in the devlopment of this model.

A seminal result was provided by Bakulin and coworkers [100], who used a sub-band gap push pulse to excite relaxed CT states shortly after photoexcitation (see Figure 1.13). The resulting increase in photocurrent gave solid evidence for relaxed CT states being bound at the interface, and for the higher excited CT states to provide the separation required for complete charge separation. Crucially, the magnitude of the signal was inversely proportional to the device quantum efficiency, highlighting the relaxed CT state as a crucial loss mechanism in OPVs that must be circumvented in order for charge dissociation to occur, rather than a precursor to separated charges. 

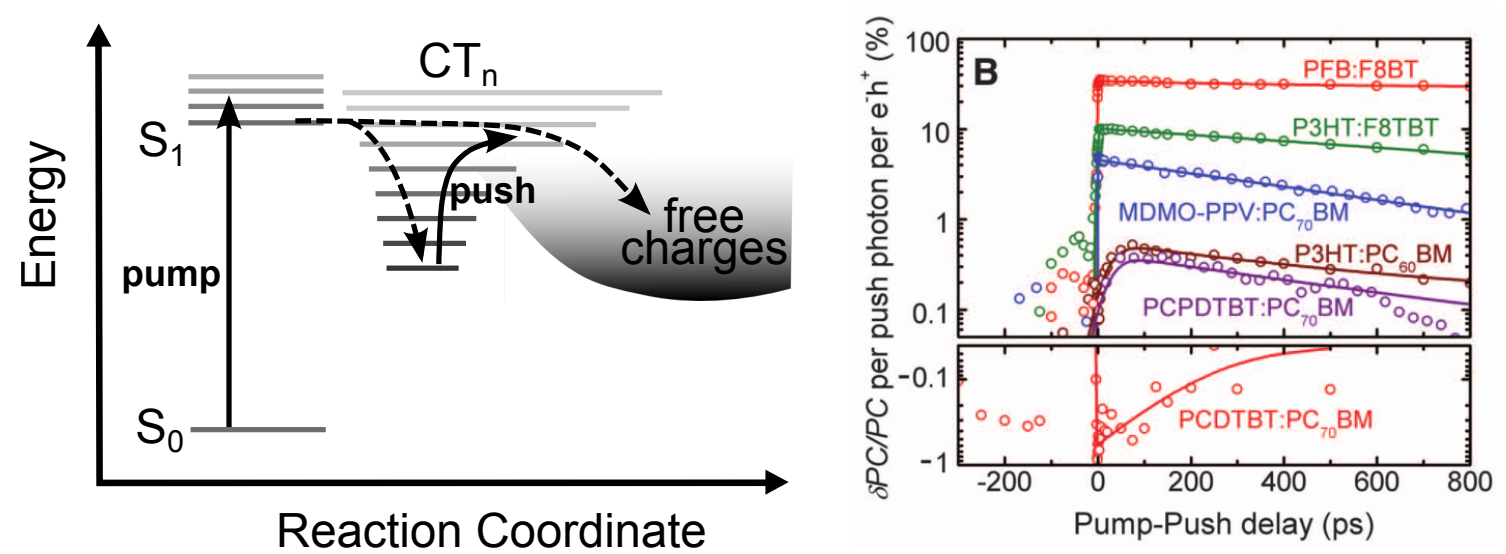

Figure 1.13: Reexcitation experiment, in which a sub-bandgap ('push') pulse arrives after the initial excitation, reexciting thermalized charge pairs and increasing the photocurrent received at the electrodes. It was found that inefficient blends produced a larger push photocurrent, implying that bound charge-transfer states represent a significant loss channel in these materials (reproduced from [100]).

The localization and trapping of $\mathrm{CT}$ states at the interface implies a branching of populations occuring on the thermalization timescale. As both populations of charges may exhibit very similar or identical spectroscopic signatures, crafty methods are required to quantify branching ratios and rates.

Since the bound charges are immobile at the interface, it is reasonable to assume that they recombine geminately, which is a monomolecular process as it involves species created from a single photon. Free charges however, are mobile and likely to recombine bimolecularly, at a rate that is strongly dependent on total charge density. Howard et al. have used this dependence to good effect by measuring recombination under various excitation densities. [101,102] A kinetic model that assumes initial branching of the populations provides a good fit, enabling measurement of the branching ratio which was revealed to be dependent on sample morphology. In P3HT:PCBM, the fraction of free charges was found to improve from 0.68 to 0.85 upon annealing. This method is used extensively in our work in Chapters 4 and 5, which further supports the branching model.

Evidence for the delocalization of excitons was provided recently by the very talented Chen and his handsome coworkers, [98] in an experiment which exploited the tendency of excitons to interact with each other and annihilate. The resulting quenching of photoluminescence and its dependence on exciton density was used to make measurements of the volume of these excitations, revealing initial exciton 
volumes approaching $20 \mathrm{~nm}^{3}$ and high mobility correlated with efficient charge generation on a time scale competitive with structural relaxation.

Recent studies of electro-absorption effects in the absorption spectra of excitons in the presence of separated charge pairs suggests that charges are separated by $4 \mathrm{~nm}$ within $40 \mathrm{fs}$ of excitation, which is attributed to a brief period of band-like charge transport accessible prior to relaxation of the CT state. [35] Additional evidence against the role of an intermediate CT state comes from time-resolved resonance-Raman spectroscopy of efficient OPV blends, in which charge generation takes place without the vibrational relaxation that would indicate structural changes associated with formation of an interfacial CT state. [103]
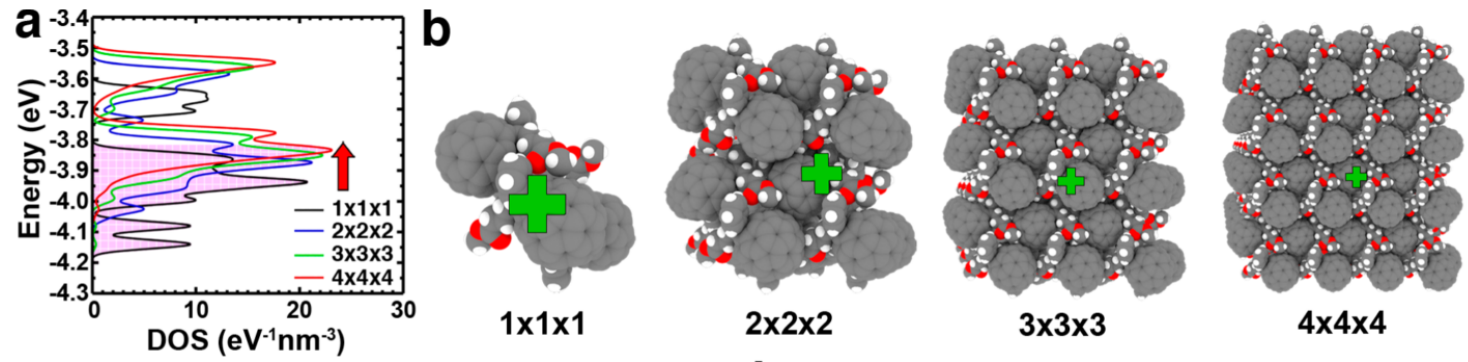

Figure 1.14: Computational models of the density of states in PCBM crystallites show reducing trap character with increasing cluster size (reproduced from [104])

Computational models of charge separation are evolving along with experiment, and in particular have shown that single dimer models of the donor acceptor interface are insufficient to replicate the rapid and efficient charge generation and separation. Rather, it is the extended donor and acceptor structure that forms the manifold of delocalized excited states that enable the surprisingly efficient charge separation in these otherwise tightly bound materials (see Figure 1.14). [104,105]

It has further been proposed that the broadened density of states leads to extremely high (non-equilibrium) initial charge carrier mobilities that are sufficient for charge pairs to rapidly diffuse apart on thermalization time scales, [106,107] with Monte Carlo models decribing this entropically driven diffusive transport able to explain the observed rates of charge carrier formation and recombination. Furthermore, work from Caruso and Troisi suggests that excitons can be dissociated at long-range (tens of angstroms) from interfaces, reducing the barrier for the electron to tunnel across the interface to the acceptor. [108]

However, in spite of the emergence of models describing different relationships between excitons, CT states and SCs on a potential energy surface spanning sev- 
eral nanometers from the donor/acceptor interface, critical parameters including distances have yet to be experimentally established.

\subsection{Spectroscopy as a probe of charge photogenera- tion}

The frontiers of our understanding as described in Section 1.3.3 have been notably driven by spectroscopic methods. Time-resolved spectroscopy can now be carried out with sufficient sensitivity and time resolution to comprehensively map out kinetic models connecting species on vastly disparate time scales, [102] and new types of multiple pulse measurements have successfully identified extremely short-lived states that play a key role in photocurrent generation. [100]

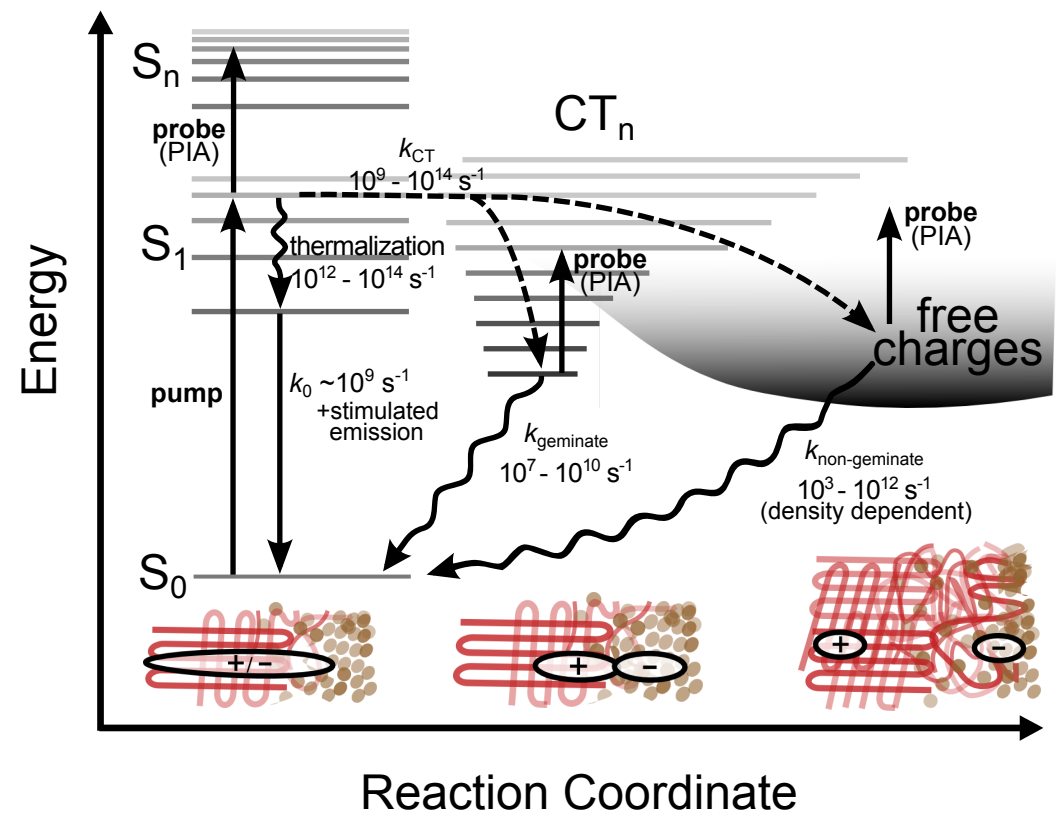

Scheme 1.1: Photophysical scheme showing the relevant processes and characteristic timescales for photocurrent generation in OPV films.

This thesis focuses primarily on the application of pump-probe transient absorption (TA) spectroscopy, a technique which probes changes in absorption due to the presence of excited states in a material (introduced in detail in Section 2). From our review of the state of the literature in Section 1.3.3, we can draw a picture of the range of interactions and associated rates that may be probed with TA to give insight into the photoseparation process (Scheme 1.1). 
Transient absorption (TA) spectroscopy is ideally suited to probing the physics of photocurrent generation in OPVs. The variety of different optical excitations involved (e.g., singlet and triplet excitons, charge-transfer states, and polarons) all present spectroscopic signatures throughout the visible-infrared regions as well as sensitivity to their environment (e.g., polymer morphology, temperature). From our review of the state of the literature in Section 1.3.3, we may draw a picture as shown in Scheme 1.1 showing the range of interactions and associated rates that may be probed with TA to give insight into the photoseparation process.

As depicted in Scheme 1.1, transitions between these states can occur on timescales ranging from tens of femtoseconds for charge photogeneration in polymerfullerene OPVs [96] to microseconds and beyond for charge recombination. [102] Moreover, since free charges can recombine bimolecularly, the recombination dynamics are dependent on excitation density. Likewise, excitations existing earlier in the photophysical cascade are also subject to bimolecular reactions at sufficiently high excitation densities, including exciton-exciton annihilation or exciton-charge annihilation. [109] The importance of bimolecular reactions motivates the need to employ excitation intensity dependence as a standard tool in OPV spectroscopy and also constrains the excitation intensity that should be used to resolve inherent monomolecular processes. For example, an excitation fluence on the order of only $1 \mu \mathrm{J} \mathrm{cm}^{-2}$ produces an initial excitation density of only $10^{17} \mathrm{~cm}^{-3}$ for a typical OPV film on the order of $100 \mathrm{~nm}$ thick and with an absorption coefficient of $2 \times 10^{4} \mathrm{~cm}^{-1}$. This excitation density is on the upper end of the steady-state excitation density expected for an OPV device operating under solar illumination, $[37,102]$ yet the TA signal is expected to be only $2 \times$ $10^{-4}$, assuming TA cross-sections on the order of $2 \times 10^{-16} \mathrm{~cm}^{2}$. This calculation, and similar consideration of faster bimolecular exciton annihilation reactions, immediately dictates the need for TA sensitivity better than $10^{-5}$ when studying photocurrent generation in OPV films.

\section{Challenges for OPV spectroscopy}

Many of the outstanding questions regarding the photogeneration process relate to distance scales. What is the spatial extent of the exciton, is it time dependent and does delocalization play a role in the extremely fast charge transfer that follows photoexcitation? And what is the spatial distribution of the charge pair following charge transfer? Is dissociation beyond the Coulomb radius achieved by the charge 
transfer process itself, or via a bound CT state? While the time scales of processes associated with charge generation are readily measured by ultrafast spectroscopy, distance scales of excited states are more difficult to determine.

This thesis describes the development of spectroscopic tools and techniques for quantifying fundamental parameters, such as length scales, associated with the charge generation process in OPVs.

Chapter 2 of this thesis describes the construction of an ultra-sensitive TA spectrometer designed specifically for investigating charge generation in OPVs, and introduces its application in the study of charge generation in a new lowbandgap polymer. This is followed in Chapter 3 where we present an investigation of noise sources in ultrafast spectroscopy, and introduce a novel application of highly-chirped probe pulses to improve the sensitivity of broadband ultrafast TA.

In Chapter 4 we introduce a range of OPV blends of varying efficiency that are used in several experiments. Density dependent measurements of charge recombination are used to determine the yields of free charge carriers in each blend, which is used to confirm the accuracy of a new technique introduced in the following chapter.

In Chapter 5 we investigate the distance distributions of photogenerated charge pairs immediately following the charge transfer process, revealing bimodal distributions of separated charge disances that imply a critical distance of $\sim 3-4 \mathrm{~nm}$ for free charge generation.

Finally, Chapter 6 describes the use of probe intensity measurements to make time-resolved measurements of absorption cross-section and excited state population in conjugated polymers. The ability to measure these parameters could prove extremely useful in situations such as quantifying fission processes or other branching yields, determining key parameters used in fitting photophyical models, or even in illuminating the role played by ultrafast delocalization in free charge photogeneration. 


\title{
Chapter 2
}

\section{Construction of an ultra-sensitive transient absorption spectrometer}

\begin{abstract}
iThe design of effective materials for efficient organic photovoltaic cells requires developing a detailed photophysical model of the processes that link photon absorption to photocurrent collection. Transient absorption spectroscopy offers the potential to do so, but its value depends on the ability to carry out measurements with sensitivity of better than $10^{-5}$ and from femtosecond to microsecond timescales (as discussed in Secion 1.4).

In this chapter, we describe the transient absorption spectroscopy tools that we have developed specifically for probing charge photogeneration and recombination in organic photovoltaic cells, and we illustrate their implementation by investigating the factors limiting charge photogeneration in a new low-bandgap polymer.
\end{abstract}

\section{An aside regarding attribution}

The beginning of this project coincided with the establishment of a new ultrafast spectroscopy lab at Victoria University of Wellington. It would be a wonderful world indeed if every budding laser physicist had the opportunity to walk into

${ }^{\mathrm{i}}$ This work has been published (with the exception of Section 2.3): Alex J. Barker, Kai Chen, Shyamal K.K. Prasad, And Justin M. Hodgkiss (2014) High-sensitivity Ultrafast Transient Absorption Spectroscopy Of Organic Photovoltaic Devices. Ultrafast Dynamics in Molecules, Nanostructures and Interfaces, pp. 36-52. 
a new lab with state of the art laser, sparsely populated optical table and carte blanche (in the figurative sense!). It is certainly an opportunity I am grateful for. However, it is worth noting that development of the spectrometer and associated tools (including optical layout \& related hardware, electronics, and control and analysis software) constitutes a significant fraction of the total effort towards the realization of this thesis, and some mention of the people involved is merited.

The TA spectrometer was built under the supervision of Justin Hodgkiss by myself with Kai Chen, who was a fellow PhD student working in parallel to develop our ultrafast photoluminescence spectrometer. $[98,110]$ The framework of the LabVIEW control software was developed by Thomas Haberzettl, which I implemented with significant modification. The data analysis software was all written by myself in MATLAB.

\subsection{Introduction}

In principal, sensitivity can easily be improved simply by extending data acquisition times. However, more elegant and efficient means of achieving high sensitivity become extremely valuable for high throughput measurements resolving the sensitivity of dynamics to a wide range of initial conditions like temperature and excitation intensity. As we will demonstrate, a further benefit of high sensitivity is the ability to resolve the TA spectra of secondary excitations generated by reexciting an already small population of transient states. An important application of these experiments is probing the short-lived non-thermalized charge-transfer states that are found to precede the formation of free charge carriers.

With those challenges in mind, the purpose of this chapter is to give an overview of the apparatus and methods that we employ to acquire extremely high sensitivity TA data capable of resolving processes over femtosecond to microsecond timescales, and to present several case studies from our investigations of photocurrent generation in OPVs in which the benefits of high sensitivity and broad time resolution are highlighted.

\subsection{Experimental details}

TA spectroscopy employs pairs of pump (excitation) and probe pulses that spatially overlap in the sample. The time- and wavelength-dependent changes in the 
transmission of the probe pulse induced by the excitation pulse are extracted from the ground state transmission spectrum by modulating the excitation (usually at $\omega / 2$, where $\omega$ is the probe pulse repetition rate) and calculating the differential transmission;

$$
\frac{\Delta T}{T}=\frac{T_{\text {pump on }}(\lambda, t)-T_{\text {pump off }}(\lambda)}{T_{\text {pump off }}(\lambda)},
$$

where $T$ is time- and wavelength-dependent probe pulse transmission. In the small signal limit $(\Delta T / T<<1), \Delta T / T$ is proportional to both excitation density and sample thickness. $[111,112]$ Given that the TA signal is derived from the intensity differences between pairs of sequential probe pulses, it is clear that the sensitivity towards real pump induced signals depends on the ability to minimize the effect of other fluctuations between sequential probe pulses. [113]

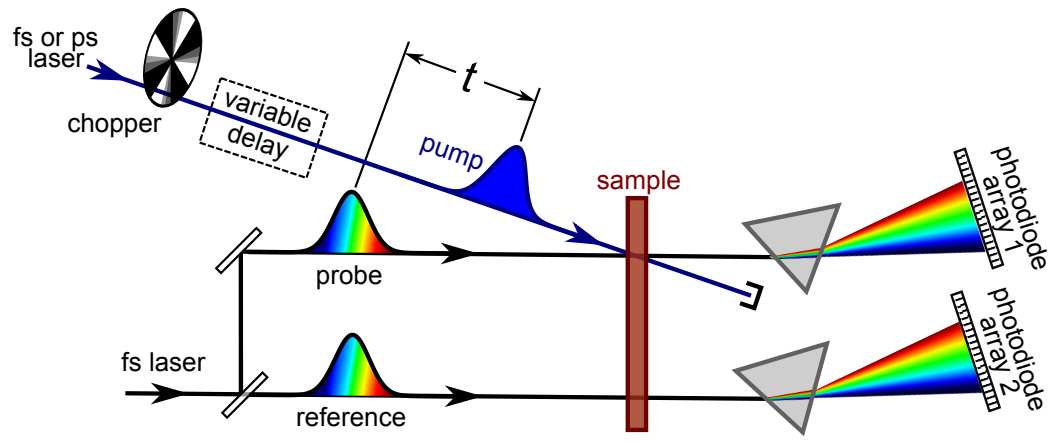

Figure 2.1: Schematic showing the main optical components of our transient absorption spectrometer. Important components not shown include wavelength conversion and broadband light generation for pump and probe pulses, and signal digitization and processing after collection on the photodiode arrays.

\subsubsection{Excitation light sources and pulse timing}

Our TA spectrometer is based on a commercial Ti:Sapphire amplifier ii that generates $100 \mathrm{fs}, 1.3 \mathrm{~mJ}$ pulses at a repetition rate of $3 \mathrm{kHz}$. This relatively high peak pulse energy allows us to drive several non-linear processes simultaneously, specifically two optical parametric amplifiers which function as tunable light sources for excitation and probing of the sample. The choice of a $3 \mathrm{kHz}$ repetition rate over the more common $1 \mathrm{kHz}$ systems allows us to collect a larger number of data

\footnotetext{
iiSpectra-Physics Spitfire Pro, with Mai Tai SP oscillator and Empower pump
} 
points in a given time, increasing sample throughput and/or effective sensitivity via averaging.

Our primary source of excitation light is a commercial travelling wave optical parametric amplifier ${ }^{\text {iii }}$ (TOPAS) capable of producing narrowband $(<1.3$ times transform-limit) $100 \mathrm{fs}$ pulses over a tunable range from $1150-2600 \mathrm{~nm}$. Frequency doubling and mixing stages at the TOPAS output extend this capability, allowing us to access a total range of excitation wavelengths spanning $240-2600 \mathrm{~nm}$.

The delay of the pump pulse relative to the probe pulse is varied using a broadband retroreflector mounted on an automated linear translation stage. As the positioning repeatability of the stage equates to an uncertainty in delay of less than $10 \mathrm{fs}$, the time resolution of our system is limited only by the pulse width of our pump and probe pulses.

As delays longer than $\sim 3.5 \mathrm{~ns}$ exceed the range of our mechanical delay stage, we excite using the second harmonic of an electronically triggered Q-switched laser $(0.7 \mathrm{~ns}$ pulse width). This laser is synchronized with the femtosecond laser via an electronic delay generator triggered from the previous femtosecond pulse to collect data from $1 \mathrm{~ns}$ to arbitrarily long pump-probe delays (although for delays longer than $300 \mu$ s this requires operating the amplifier at a reduced repetition rate). The combination of these two pump-probe delay configurations spans the femtosecond to millisecond time ranges of interest to OPVs with the same detection system and without any time gaps. [102,114,115]

The choice of pump-probe delays to be used for a given experiment is based on the need to capture all photophysical processes under investigation with sufficient time resolution, while minimizing the time required to run the experiment. Photophysical processes in OPVs are found to span a wide range of timescales, therefore we typically employ a logarithmic distribution of time points to generate data that can be fitted with an equal weighting on each time scale. The main exception to this is for regions where significant probe chirp exists, where linearly spaced time points aid in the implementation of a chirp-correction algorithm (see Section 3).

\subsubsection{Probe light sources}

To generate broadband visible probe pulses, we employ a homebuilt noncollinear optical parametric amplifier (NOPA). [116] The visible NOPA provides intense

\footnotetext{
iiiTOPAS-C, Light Conversion Ltd
} 
and stable light from $520-760 \mathrm{~nm}$. If the experiment requires broader and higher energy probe spectra, we generate unamplified white light supercontinuum as far as $300 \mathrm{~nm}$ in a thin $\mathrm{CaF}_{2}$ window. [113] The ability to probe at near-infrared (NIR) energies is especially important as low-bandgap polymers that absorb into the NIR emerge as promising materials for widespread OPV implementation. We currently generate NIR supercontinuum in a $3 \mathrm{~mm}$ sapphire window using an intense $1200 \mathrm{~nm}$ pulse from the TOPAS. However these unamplified light sources typically result in greater noise levels due to inherently lower shot-to-shot stability than the NOPA, and to an inability to fill the photodiode bins in our detector to an optimal level. We note that variations on our visible NOPA are able to generate more stable and intense light in the NIR region, [116] at the expense of bandwidth and tunability.

\subsubsection{Light detection}

The effect of probe pulse fluctuations can be minimized by employing a reference channel to monitor probe intensities on a shot-to-shot basis. Prior to interaction with the sample, the probe beam is split using a broadband beamsplitter. The reflected portion is sent through the sample, overlapping with the pump beam, while the transmitted portion is used as a reference beam. [112] After passing through the sample, the vertically displaced probe and reference beams are dispersed by a short focal length spectrometer that is selected to achieve high throughput while maintaining sufficient resolution for the broad electronic transitions observed in OPV materials. We use a fibre-coupled grating based spectrometer ${ }^{\text {iv }}$ when probing with the NOPA. A free-space coupled prism spectrometer is used when probing with unamplified light, as the higher throughput (especially at shorter wavelengths) and ability to adjust the dispersion to optimize light intensity per pixel minimizes digital readout noise as a result of low light intensity.

The probe and reference spectra are recorded using a pair of 256-pixel linear silicon photodiode arrays, ${ }^{\mathrm{v}}$ which are read out at the laser repetition rate of $3 \mathrm{kHz}$. We can optionally switch to a linear InGaAs array for probing NIR energies. Our analogue-to-digital converter has four additional analog inputs, which are used to monitor pulse intensities via additional single channel photodetectors. The

\footnotetext{
iv Princeton Instruments Acton SP150

${ }^{v}$ Entwicklungsbuero Stresing
} 
speed of our read out system enables shot-to-shot detection of complete probe and reference spectra a capability that exceeds most CCD cameras and opens the door to several noise reduction methods described below.

\subsubsection{System automation}

The brain of our transient absorption spectrometer is a modular system of LabVIEW virtual instruments that communicate with each other via National Instruments proprietary Datasocket Server protocol. The tasks performed by each module are depicted in Figure 2.2.

The goal of our data acquisition system is to provide shot-to-shot detection at $3 \mathrm{kHz}$ with as high a duty cycle as possible, i.e., minimum processing time between data collection cycles to capture that maximum amount of data in a given time. It is crucial that the software is able to keep up with this rapid rate of data collection, as we have found that digital noise can be introduced to the data as a result of timing and memory problems. To ensure optimum performance, the LabVIEW code uses standard best practice measures such as memory preallocation, an event-driven architecture, and appropriate prioritization of resources within the operating system. As the separate LabVIEW modules can communicate via Ethernet using the Datasocket Server, we dedicate one computer to the main data collection and processing role, and a second computer is used for all other roles such as user interface and data presentation.

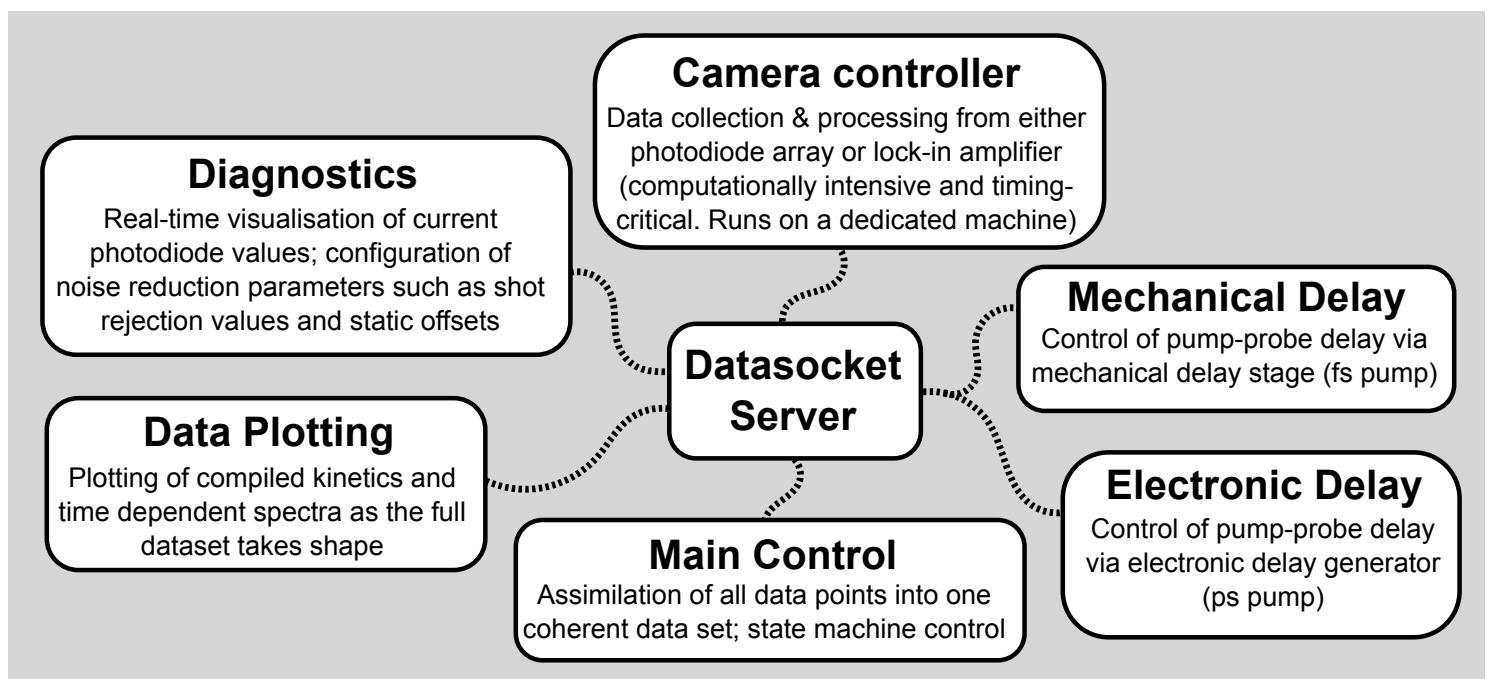

Figure 2.2: Schematic showing automation software connections. 


\section{Noise reduction during data collection}

The most significant source of noise in our system is due to wavelength-dependent fluctuations in the intensity of our pump and probe light. Non-linear processes such as those taking place in our NOPA and TOPAS are extremely sensitive to beam alignment and mode quality, as well as laboratory temperature and humidity. Therefore, in addition to implementing tight environmental stability controls, we enclose beam paths prior to non-linear processes to minimize unnecessary fluctuations due to air currents and particles passing through the beam.

The wavelength-dependent intensity fluctuations in our light sources are typically comprised of several components. Slower, breathing oscillations that take place on the order of seconds can be minimized through environmental control and corrected for using the methods described below. The remaining noise is fast shot noise, inherent to the laser itself and an unavoidable consequence of the quantized processes central to laser operation. This noise exhibits no correlation between sequential shots, and as such cannot be corrected for. Averaging is our best weapon against shot noise, with the signal-to-noise ratio scaling by $\sqrt{N}$ with our sample number $N$.

Several thousand probe shots are typically collected per pump-probe delay point. Each probe spectrum is divided by the reference spectrum in order to correct for shot-to-shot variations in probe shape. [112] An external photodiode collects scattered pump light, allowing us to correlate fluctuations in pump intensity with each collected spectrum. Additionally, the pump photodiode acts as a trigger for the $\Delta T / T$ calculation, labeling spectra pump on or pump off. Each batch of pump on and pump off spectra are separately averaged and then used to calculate $\Delta T / T$ as in Equation 2.1. This approach is less susceptible to noise from occasional large probe fluctuations than if $\Delta T / T$ values are calculated individually prior to averaging.

As the signal from the pump photodiode provides us with a measure of relative pump intensity for each collected spectrum, we are able to define a window of acceptable intensities for the experiment. Spectra taken when the pump intensity is outside the window are rejected and do not contribute to the averaged spectrum for each time point. This is particularly valuable when pumping with the TOPAS, which is generally stable (within specification) but exhibits occasional slow fluctuations that persist for longer than the several thousand shots comprising each time point and are therefore not effectively averaged out. 
For a typical high-sensitivity measurement, we conduct several runs through the entire time series before implementing the post-processing measures outlined in the next section. Finally, we have developed a new method that exploits chirped pulses in order to acquire very high sensitivity kinetics, which is described in Section 3.

\section{Noise reduction after measurement: Data processing}

Once the measurement is complete, the collected data is processed in MATLAB. The results of each pump-probe delay scan are compiled, and compared to check for effects from photodegradation and experimental drift, before being averaged into a single wavelength- and pump-probe delay-dependent array of $\Delta T / T$ values.

The next step is to apply a transformation to correct for the 'chirp' of our system - the wavelength-dependent value of pump-probe delay corresponding to temporal overlap of our pump and probe pulses in the sample, $t_{0}(\lambda)$, where the wavelength dependence is primarily due to the net group-velocity dispersion of the broadband probe pulse in our experiment. For each experimental configuration, a single run is taken at high pump intensity, such that a strong and fast (within instrument response) TA signal or alternatively, coherent artifact [117] is observed at $t_{0}$. Points are manually entered along the artifact using a graphical interface, allowing us to fit a 3rd degree polynomial corresponding to the net chirp of our system. The dataset is temporarily upsampled (linear 1-D interpolation of the data for each wavelength value), the appropriate temporal offset is applied for each wavelength, and the chirp-corrected dataset is downsampled back to a common set of pumpprobe delays.

Any static features (that may exist, for example, from scattered pump light incident on the photodiode array) are removed by subtracting from the entire dataset the average of the spectra collected at $t<t_{0}$. A smoothing filter is then typically applied to the individual spectra, as the effective wavelength spacing of our photodiode pixels exceeds the wavelength resolution of our spectrometer, which is optimized for light throughput.

Finally, other signal recovery or data fitting techniques may be applied as appropriate for the experiment in order to determine the ensemble of lifetimes and spectral contributions contributing to the observed signal, and to deconvolute these from the instrument-response function. Techniques often used include singular value decomposition, global fitting, genetic fitting algorithms, and 
maximum-entropy methods.

\title{
Measuring beam profiles
}

In order to determine the spatial distribution of pump and probe beam energy density, we place the exposed CMOS detector from a common webcam ${ }^{\text {vi }}$ in the sample plane of our experiment. Using the published pixel size and pitch of the CMOS elements, gaussian fits of the data give us the FWHM of each beam for the purposes of estimating excitation density within the sample, or for any data fitting that requires knowledge of incident distribution of excitation density.

\subsection{Case study: Charge generation in PTBT:PC ${ }_{61} \mathrm{BM}$}

To give an example of the results generated by our TA spectrometer, we present here an investigation of a recently developed low-bandgap polymer PTBT. ${ }^{\mathrm{vi}}$

\begin{abstract}
TA spectroscopy of PTBT:fullerene blends reveals high internal charge-transfer yield and highlights morphology-dependent charge recombination as the main factor limiting device photocurrent.
\end{abstract}

\section{Introduction}

It was previously reported that PV cells constructed using PTBT have a high open circuit voltage $\left(V_{\mathrm{OC}}\right)$ but suffer from only a moderate short circuit current density $\left(J_{\mathrm{SC}}\right)$ as a result of $50 \%$ external quantum efficiency throughout the visible spectrum. [118] TA allows optical probing of the density of photoexcitations such as excitons and charges over a large range of time scales (in this study $10^{-13}-10^{-4}$ seconds after excitation) without relying on the transport of charges to electrodes. It is therefore an ideal method by which to investigate the factors limiting $J_{\mathrm{SC}}$.

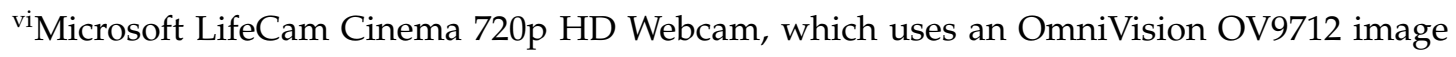
sensor with $3.04 \mu \mathrm{m}$ pixel pitch

vii This work was undertaken as part of a wider study of PTBT, in collaboration with the groups of Keith Gordon (University of Otago, New Zealand) and Han Young Woo (Busan National University, Miryang, Republic of Korea). Manuscript in preparation.
} 
Additionally, the spectral signatures of these excitations give us insight into their local environment - in this case, to the morphology of their host molecules.

\section{Experimental}

TA spectroscopy was performed on three thin film samples: ${ }^{\text {viii }}$ neat PTBT, PTBT:PC ${ }_{61}$ BM spin-cast from chlorobenzene (CB), and PTBT:PC ${ }_{61} B M$ spun from a mix of $\mathrm{CB}$ and 1, 8-octanedithiol (CB+ODT, 98:2 v/v).

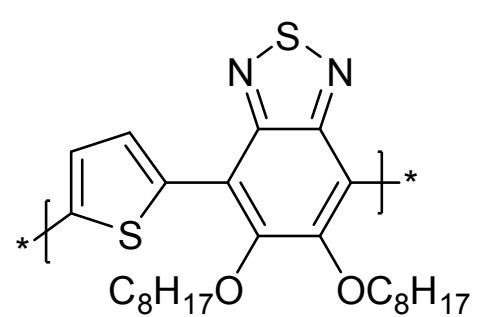

(a) PTBT structure

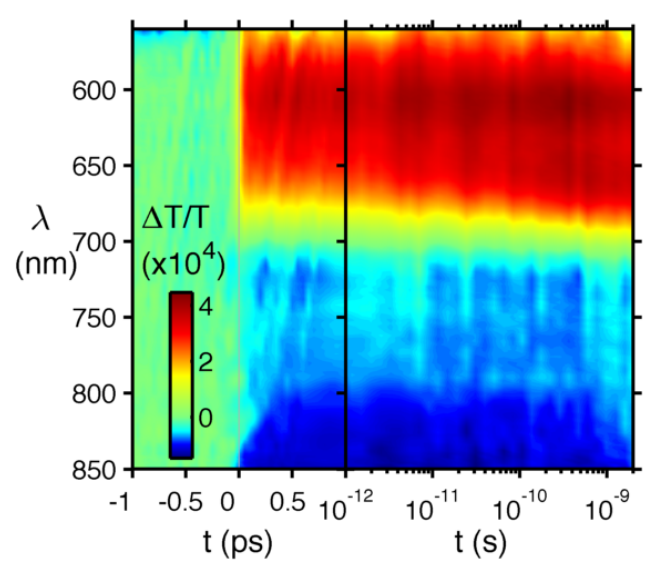

(c) PTBT:PC ${ }_{61}$ BM from $\mathrm{CB}$

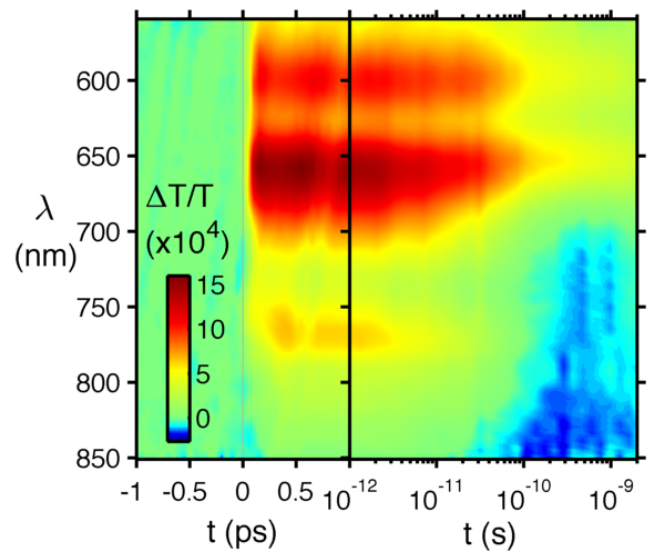

(b) neat PTBT film

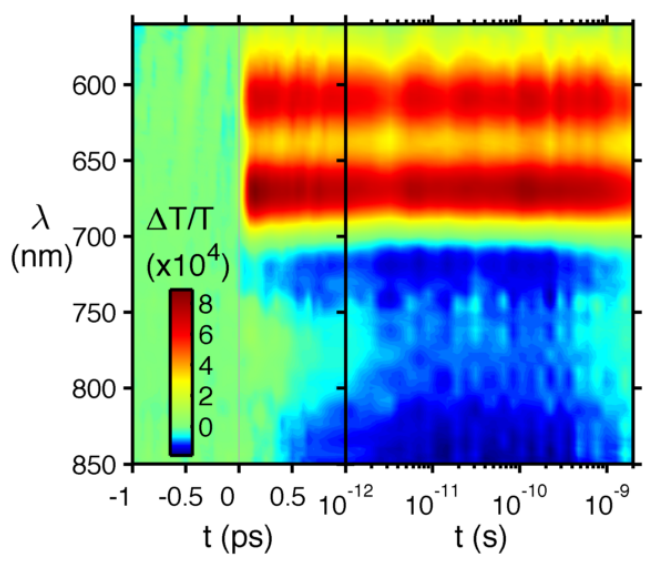

(d) PTBT:PC ${ }_{61} \mathrm{BM}_{\text {from } \mathrm{CB}+\mathrm{ODT}}$

Figure 2.3: (a) PTBT structure, (b)-(d) TA maps of PTBT and PTBT:PC ${ }_{61} B M$ films. Samples were excited with $100 \mathrm{fs}, 532 \mathrm{~nm}$ pulses at $5 \mu \mathrm{J} \mathrm{cm}{ }^{-2}$ pulse $^{-1}$.

\footnotetext{
viii PTBT was kindly provided by the Han Young Woo group at Busan National University. $\mathrm{PC}_{61} \mathrm{BM}$ was purchased from Sigma-Aldrich.
} 
Processing with a small amount of alkane dithiols has been shown to increase domain size and polymer self-organization (and therefore $J_{\mathrm{SC}}$ ) in PTBT:PCBM [118] and other polymer:fullerene blends. [60] This process is comparable to the well-known effects of thermal and solvent vapour annealing in commonly studied materials such as P3HT:PCBM. [72]

Measurements were performed using the experimental apparatus described earlier in this chapter, with broadband probe light from $550-850 \mathrm{~nm}$ generated in our NOPA (described in Section 2.2.2). For ultrafast measurements, an optical parametric amplifier (TOPAS) provided $100 \mathrm{fs}$ excitation pulses at $532 \mathrm{~nm}$, which were chopped at $1.5 \mathrm{kHz}$. Excitation energy density at the sample was kept at $5 \mu \mathrm{J} \mathrm{cm}^{-2}$ pulse $^{-1}$. Intensity-dependent measurements were performed and found no variability in the ultrafast spectra, confirming that our excitation density was sufficiently low that non-linear artifacts such as exciton-exciton or exciton-charge annihilation were negligible. $[78,109,119]$ The relative delay between pump and probe pulses (up to $3 \mathrm{~ns}$ ) was varied using the broadband retroreflector mounted on an automated delay stage.

\section{Assigning spectral features}

In order to analyze the TA maps shown in Figure 2.3, we first consider the spectral shape of the signals by taking slices ('spectra') at several time ranges. Figure 2.4 shows TA spectra of each film at selected times after photoexcitation. By comparison with the steady-state photoluminescence, [118] we can identify the positive differential transmission $(\Delta T / T)$ signal centered at $770 \mathrm{~nm}$ as stimulated emission (SE), allowing us to track the exciton population. Photoinduced polarons are non-emissive but do absorb light, corresponding to the photoinduced absorption (PIA, negative $\Delta T / T$ ) at wavelengths beyond $700 \mathrm{~nm}$.

The positive ground-state bleach (GSB) signal from $560-700 \mathrm{~nm}$ is due to both excitons and polarons, and reflects the UV-vis spectrum of the material that those species occupy.

Distinct vibronic structure is present in the TA spectrum of PTBT, indicating significant ordering and long conjugation length due to the interchain interactions present in well aggregated polymers. [120,121] Other main points to note in Figure 2.4 are strong quenching of the PTBT stimulated emission upon addition of PCBM, the appearance of long-lived excitations in the PCBM blends, and a loss of vibronic structure in the "CB only" blend (indicating disorder of the polymer chains) that 
is regained when casting films with a small amount of ODT.

Many of these spectral assignments are informed by drawing on the large body of work on well characterized materials such as P3HT and MEH-PPV, and their blends with PCBM. Many of the behaviours (such as the relationship between vibronic character and morphology, and the correspondence between steady-state photoluminescence and excitonic SE) are common to the entire family of materials, enabling TA to be used as a rapid and versatile characterization tool.

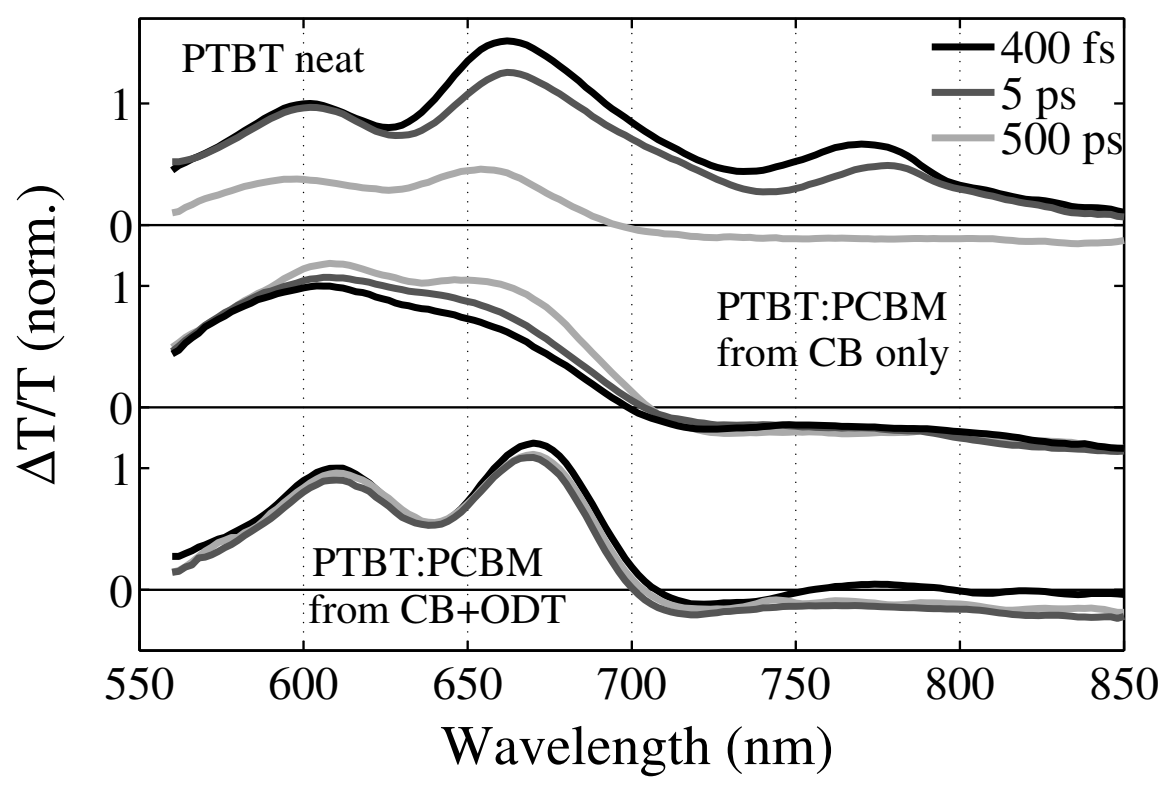

Figure 2.4: Transient absorption measurements of neat and blended PTBT thin films, excited at $532 \mathrm{~nm}$. Spectra at selected times, normalized to the peak near $600 \mathrm{~nm}$ at $400 \mathrm{fs}$.

\section{Interpreting kinetics}

The decay of the PIA signal in the PTBT film (Figure 2.5, black circles) reveals an exciton lifetime of approximately 40 ps in PTBT. Efficient charge separation requires that the rate of charge transfer be sufficient to compete with exciton decay. Indeed, the complete absence of SE in the blend from CB (Figure 2.5, blue circles) indicates that all excitons are promptly converted into charges within our instrument response ( $<200 \mathrm{fs}$ ). In the blend from $\mathrm{CB}+\mathrm{ODT}$ the majority of charges are generated promptly, however some fraction of excitons is quenched at a slower rate, over several ps (Figure 2.5, red circles). While the wavelengths used here 
give a good indication of the relative exciton and charge populations, the overlap of their respective signals in the visible region prevents us from unambiguously separating the two contributions. We have since extended our probing range into the infrared $(850-1700 \mathrm{~nm})$, where exciton and charge PIA can be clearly distinguished.

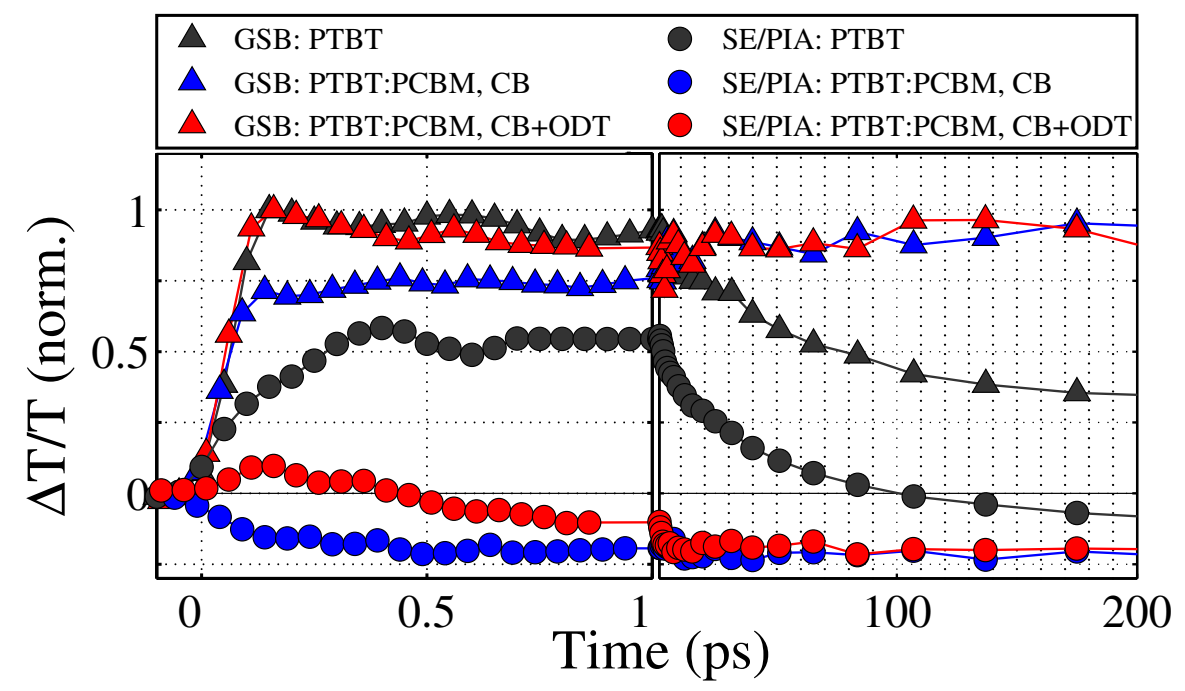

Figure 2.5: Kinetics of ground state bleach (GSB, 550-700 nm) and overlapping stimulated emission/photoinduced absorption (SE/PIA, 760-785 nm) signals.

We can estimate the fraction of prompt vs. delayed charge generation in the $\mathrm{CB}+\mathrm{ODT}$ blend by using the $\mathrm{SE}$ band at $760-785 \mathrm{~nm}$ to compare exciton population at early times ( $400 \mathrm{fs}$ ) with the neat PTBT sample (Figure 2.4, top and bottom panels). To account for the overlapping photoinduced polaron absorption at those wavelengths, we take as our baseline the $\mathrm{CB}+\mathrm{ODT}$ blend spectrum at $500 \mathrm{ps}$ (rescaled using the GSB peak at $610 \mathrm{~nm}$ as a reference), which we know contains no excitonic contribution. We accordingly find that delayed charge generation in the $\mathrm{CB}+\mathrm{ODT}$ blend results from $25 \%$ of the initial photoexcitations, which we assign to excitons generated within the larger polymer domains that must diffuse to the heterojunction interface before being quenched.

While there is a significant fraction of diffusion-limited charge generation in the $\mathrm{CB}+\mathrm{ODT}$ blend, it is important to note that there is negligible loss of GSB intensity in either of the blends during the charge-transfer process, and that the signal is maintained over a time scale much greater than the exciton lifetime. This indicates near-unit efficiency of charge transfer in both blends, revealing that this 
step is not the reason for the lower-than-expected $J_{\mathrm{SC}}$.

The ground-state bleach (GSB) from $550-700 \mathrm{~nm}$ is a result of excited chromophores not participating in ground state absorption. It therefore reflects the UV-vis absorption spectra of those excited molecules, giving us a probe of structure at the sites occupied by excitations within the material. Interestingly, we see an increase in vibronic structure in the CB blend after several hundred ps (Figure 2.4, middle panel), indicating that holes in the polymer undergo energetically driven charge transport to more ordered regions within the film. This energetic disorder is not present in the more crystalline $\mathrm{CB}+\mathrm{ODT}$ blend, consistent with both our interpretation of the kinetics and with the body of work describing the dependence of performance on morphology in materials such as P3HT:PCBM. [72,77, 102]

It is also interesting to note that in the neat PTBT film, approximately $35 \%$ of the GSB and PIA persists after several hundred ns (Figure 2.4, top panel), significantly longer than the exciton lifetime. This can be attributed to direct charge generation within the polymer with an efficiency of approximately $30 \%$, which is not without precedent. $[69,122-124]$ There is strong evidence that energetic disorder at boundaries between crystalline and amorphous regions can act as charge dissociation sites in neat polymer films. [125,126]

\section{Studying recombination with long time delay TA}

Given that our ultrafast measurements of charge generation do not account for the low $J_{\mathrm{SC}}$ in PTBT:fullerene blends, we turn to charge recombination. Recently published models of charge generation in OPVs feature an intrinsic branching at the charge-transfer event between bound- and free charge pairs, [100,102] where the former are Coulombically bound at the interface, resulting in monomolecular (geminate) recombination, and the latter are able to move throughout the film, resulting in a higher proportion of bimolecular recombination. As these two modes of recombination scale differently with charge density, intensity-dependent measurements of charge recombination provide an effective assay of the relative proportion of bound vs. free charges, [102] a technique that we will use extensively in Chapters 4 and 5.

As the time scale of interest for recombination requires pump-probe delays beyond the range of our mechanical delay stage, we use a second laser as an excitation source, as described in Section 2.2.1. Our Q-switched Nd:YVO4 laser generates 700 ps pulses at $532 \mathrm{~nm}$ (when frequency-doubling the fundamental 
output of $1064 \mathrm{~nm}$ ). The pump-probe delay was varied (ns - ms) using an electronic delay generator, and excitation density was varied as indicated in the figures.

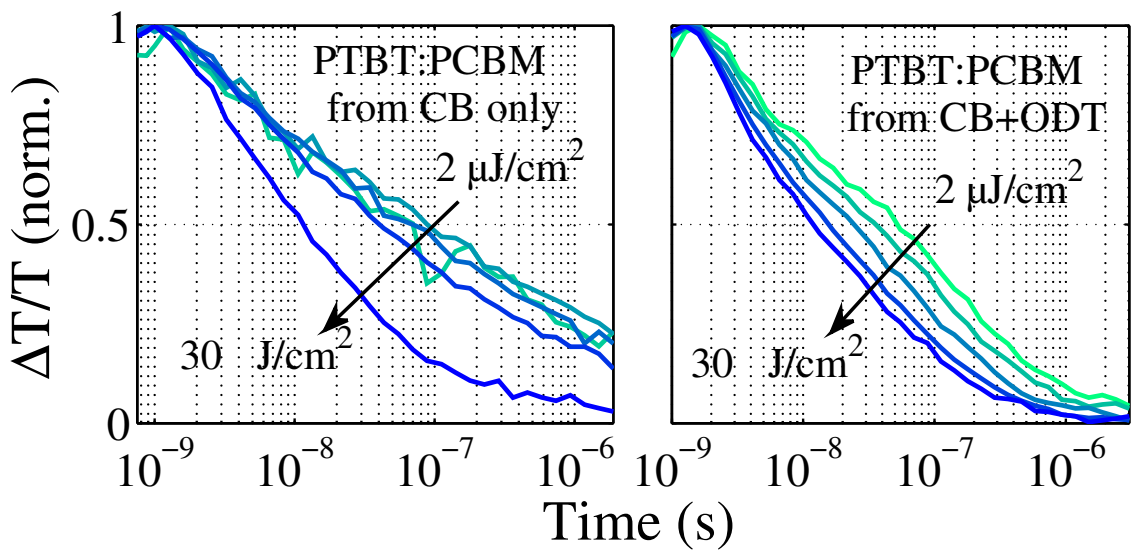

Figure 2.6: Intensity-dependent GSB decay showing charge recombination kinetics in the two blends (with excitation energy densities of $2,4,8,15$, and $30 \mu \mathrm{J} \mathrm{cm}^{-2}$ ).

Figure 2.6 shows decay of the GSB for the two blended films up to several microseconds after photoexcitation. Due to spectral shifts in the near-infrared signal (described in a publication currently in preparation) we could not apply the kinetic model from Howard and coworkers ( [102], also see Chapter 4). However we can qualitatively discuss the relative dependence of recombination on excitation density.

It is clear that recombination in the amorphous CB blend exhibits almost no dependence on excitation intensity (except for at the highest excitation value, where even bound charges can be forced to interact). This monomolecular recombination (with a lifetime of approximately $70 \mathrm{~nm}$ ) reflects high geminate recombination from bound charge pairs in regions where the blend is too finely dispersed to allow complete charge separation.

In stark comparison, the ordered $\mathrm{CB}+\mathrm{ODT}$ blend displays a clear dependence of recombination rate on charge density, indicating a greater yield of free charges. The free charge yield can be attributed to improved polymer organization, and along with a more beneficial structure for charge extraction, explains the improved $J_{\mathrm{SC}}$ in devices cast from CB+ODT. [118] At the lowest excitation densities, biphasic recombination is evident. The faster component ( $25 \%$ of the amplitude) exhibits weaker intensity dependence and is likely to reflect geminate recombination and thereby limit external quantum efficiencies and $J_{\mathrm{SC}}$ by around $25 \%$. The overall 
recombination lifetime of bound and free charges is still only $50 \mathrm{~ns}$. This lifetime is somewhat short in comparison to other materials (> $100 \mathrm{~nm}$ in P3HT:PCBM [102], $>300 \mathrm{~nm}$ in PCPDTBT:PCBM [101]). Since charge extraction must compete with recombination, this short timescale further reduces the achievable $J_{\mathrm{SC}}$ in the optimized blend. 


\title{
Chapter 3
}

\section{Ultrasensitive spectroscopy via highly-chirped broadband probes}

\begin{abstract}
Improvements in the signal sensitivity of time-resolved spectroscopy will enable key measurements relevant to open questions in many fields such as organic electronics. We show (contrary to conventional wisdom) that increasing the chirp of ultrafast broadband probe pulses can improve the sensitivity of transientabsorption spectroscopy by decorrelating noise from wavelength when measured on a multichannel detector. We show that a tenfold (or greater) improvement may be obtained in the sensitivity of kinetics obtained using highly chirped probe pulses, with no significant degradation of time resolution. The method is applied to study photoswitching in $[1,3]$ oxazine, and charge generation in organic photovoltaics with sensitivity in differential transmission on the order of $10^{-6}$.
\end{abstract}

\subsection{Introduction}

The key to unlocking many emerging TA applications is improving signal sensitivity (the ability to detect small signals) across a wide range of probe energies. Clear examples include gas-phase TA [127] where the sample is extremely dilute, and in TA microscopy of nanostructures $[128,129]$ where the sampled volume must be tiny, and many measurements need to be conducted in a reasonable timeframe in order to build a spatial map of excited-state dynamics. 
As signal amplitude is proportional to pump pulse intensity, greater sensitivity enables TA under weaker excitation. This is crucial for the investigation of photosensitive samples such as proteins [130] and historic artwork, [131] or of samples where nonlinear behaviour places constraints on excitation densities, such as the study of OPVs. In the latter case, bimolecular charge recombination, exciton-exciton and exciton-charge annihilation can result in recombination dynamics that are strongly dependent on excitation density. For a $100 \mathrm{~nm}$ thick OPV film with absorption coefficient of $2 \times 10^{4} \mathrm{~cm}^{-1}$ and excited state cross-section of $2 \times 10^{-16} \mathrm{~cm}^{2}$, the TA signal would be only $2 \times 10^{-4}$ at an excitation density of $10^{17} \mathrm{~cm}^{-3}$ - a very small signal, yet measured at the upper limit expected under solar illumination. [37] Polarization anisotropy [132] and higher-order techniques that re-excite fractions of an initial excited state population [100,133] prove even more challenging, as they rely on measuring the difference between two already small signals, but are subject to the same constraints on excitation density.

Modern TA experiments conducted using broadband probe sources do not in general experience the full benefit of multichannel detection, as noise from probe fluctuations is highly correlated with wavelength. Contrary to conventional wisdom, a highly chirped probe pulse can be beneficial to TA spectroscopy by decorrelating noise and wavelength. We demonstrate that a tenfold (or greater) improvement to the sensitivity of TA kinetics can be obtained through the exploitation of this effect in a simple and widely applicable implementation.

\subsection{Noise sources in transient absorption spectroscopy}

\subsubsection{Analysis}

Noise existing within a spectroscopic experiment can be broadly characterized as being either proportional to or independent of the signal intensity. In a transient absorption spectrometer, causes of the former include variations in pump intensity or pointing, and photodegradation of the sample. These variations reduce the signal-to-noise ratio (SNR) of the spectrometer.

Noise that is independent of signal intensity partially determines the SNR, but more directly represents a fundamental noise floor for a given experimental configuration. Fluctuations in the probe light, and processes taking place within the light detection and digitization pathway are the primary contributors to the 


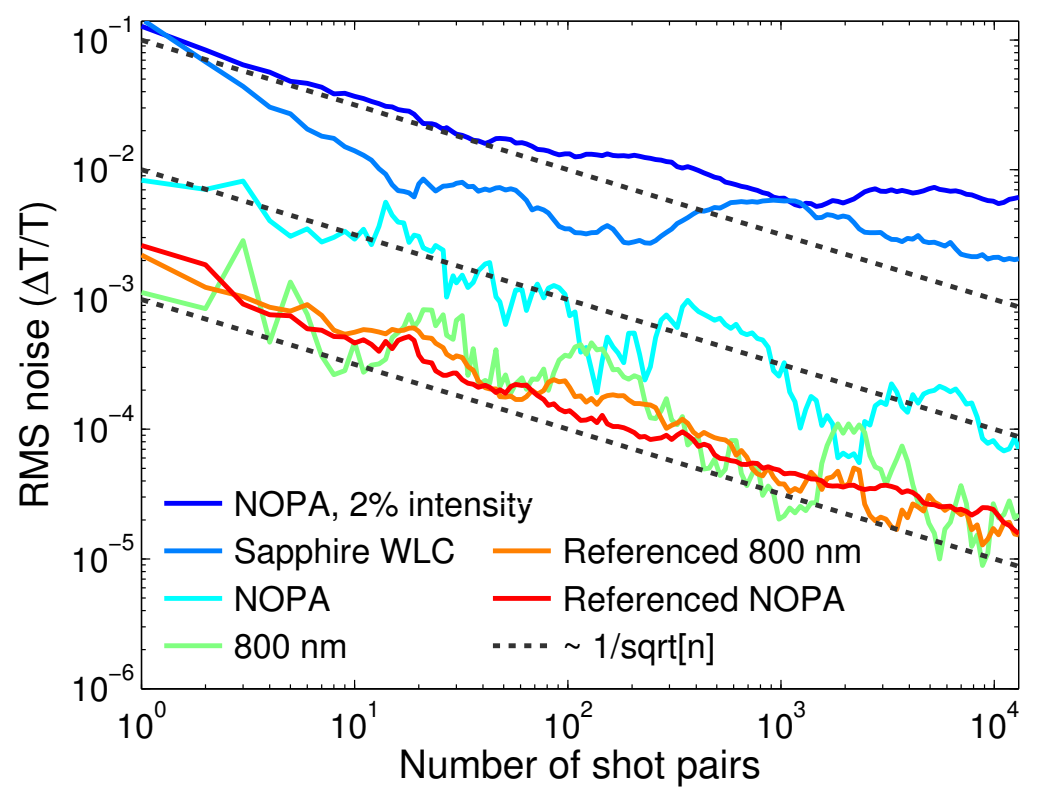

Figure 3.1: Dependence of noise floor on shot number for various narrow and broadband probes.

noise floor.

We investigated the nature of noise generated within our transient absorption spectrometer by collecting measurements in the absence of a sample or excitation light. In this situation the differential transmission $(\Delta T / T)$ should be equal to zero.

The measured deviation from zero for a given experimental configuration represents the noise floor of that experiment, and is not proportional to the signal intensity. We quantify the noise floor by taking the RMS deviation from zero ${ }^{\mathrm{i}}$ of $\Delta T / T$ for a given number of shot pairs (as two laser shots are required to calculate a single $\Delta T / T$ value).

Figure 3.1 shows the dependence of noise floor on the number of collected shot pairs $n$ for several probe light sources. These include the fundamental of our chirped-pulse amplifier (795-805 nm), sapphire white-light continuum (WLC) generated by focusing $800 \mathrm{~nm}$ pulses into a $3 \mathrm{~mm}$ sapphire window (680$790 \mathrm{~nm}$ ), and our homebuilt non-collinear optical parametric amplifier (NOPA, $580-760 \mathrm{~nm}$ ) operating as described in reference [116]. For each probe source, a total of $2 \times 10^{4}$ sequential shot pairs were individually recorded, after which the

${ }^{\mathrm{i}}$ For a zero-mean, independent Gaussian process, RMS = standard deviation. 
mean $\Delta T / T$ was calculated over progressively larger fractions of the dataset in order to build a picture of the dependence of noise on total shot number $n$. In general we see that $(\Delta T / T)_{\mathrm{RMS}}$ is proportional to $1 / \sqrt{n}$, as we would expect for a normally distributed noise source.

Our high rep-rate photodiode array (PDA) enables us to read out each shot individually, at the cost of lower photon conversion efficiency than slower systems. As a result, read-out and digitization noise are particularly detrimental when using a low intensity probe light source or optically dense sample, as the full dynamic range of the detection system cannot be exploited. Such situations are represented respectively in Figure 3.1 by the sapphire WLC, and NOPA attenuated to $2 \%$ of typical intensity. In these cases we see both generally high noise and a deviation from the $1 / \sqrt{n}$ trend showing diminishing improvement in sensitivity when increasing the sampling time (i.e. at high shot number $n$ ).

In contrast, the use of shot-to-shot referencing [112] with a sufficiently bright light source (such as the referenced NOPA) minimises noise from probe fluctuations and read-out respectively, resulting in exceptionally high-sensitivity and well-defined $1 / \sqrt{n}$ behaviour. It is clear that in this configuration we are able to further improve the sensitivity of our experiment by increasing the sampling time and/or rate.

\subsection{Data analysis \& highly chirped broadband pulses for noise reduction}

The output of a typical time-resolved spectroscopy experiment is shown in Fig. 2 (a). We chose to probe the photoinduced absorption between 660-760 $\mathrm{nm}$ in a thin film of the extensively studied organic photovoltaic blend P3HT:PCBM. The sample was excited at $480 \mathrm{~nm}$ with exceptionally low pump energy density $\left(1 \mu \mathrm{J} \mathrm{cm}^{-2}\right.$ per pulse) in order to obtain a very weak, noisy signal. The inset in Figure 3.2(a) shows the same experiment conducted at greater pump intensity. The absorption is fairly uniform from $650-750 \mathrm{~nm}$, and is due to a combination of photogenerated excitons and charge pairs. An additional faster component from 650-680 nm exists at very early times, due to electro-absorption near the onset of the ground-state absorption. [35, 123]

The kinetics of the photoinduced absorption are visualised by taking the mean 


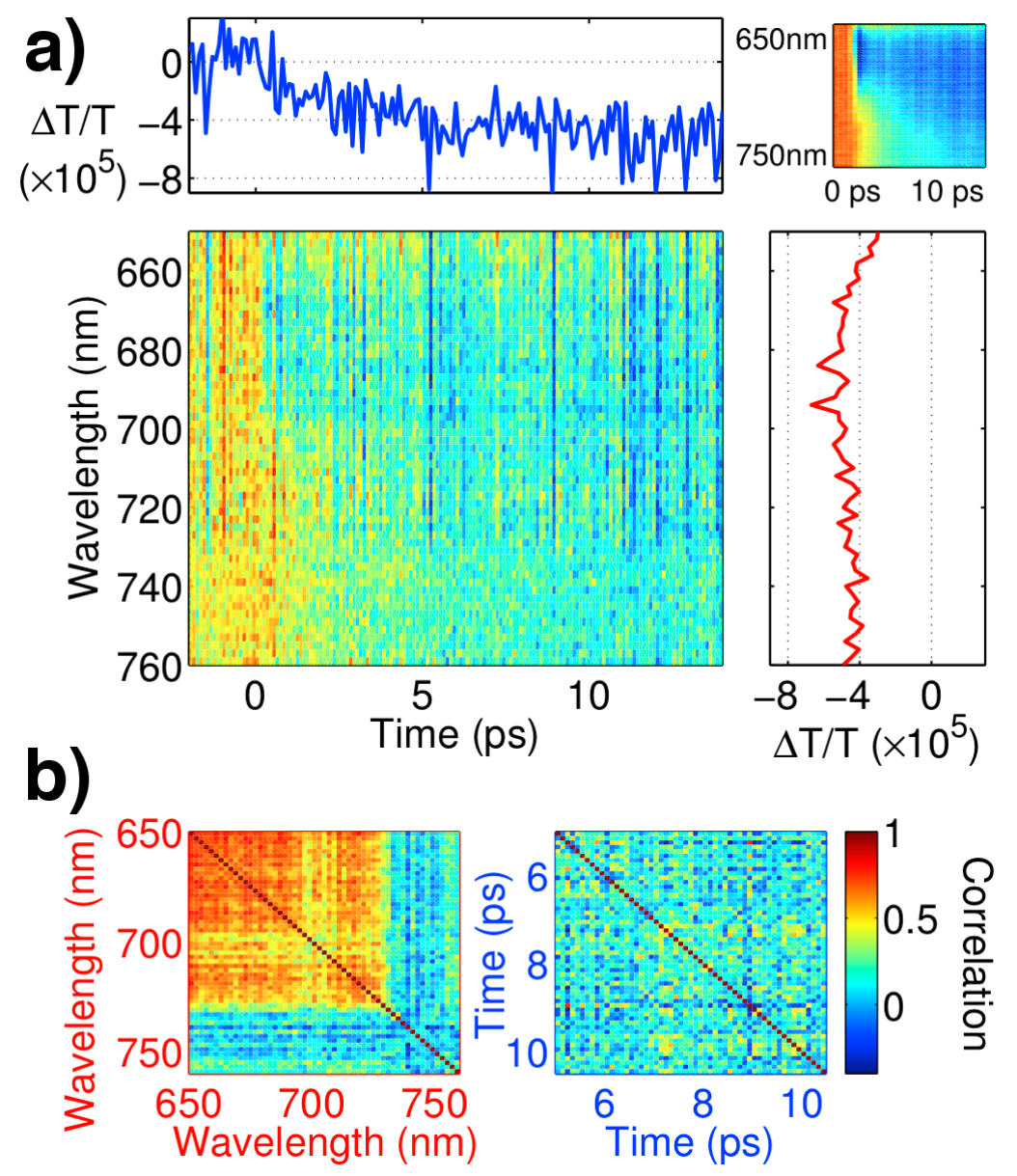

Figure 3.2: (a) TA surface for P3HT:PCBM under weak excitation $\left(1 \mu \mathrm{J} \mathrm{cm}{ }^{-2}\right)$ with kinetics (blue), spectrum (red), and (insert) TA surface collected under strong $\left(21 \mu \mathrm{J} \mathrm{cm}^{-2}\right)$ excitation. (b) Correlation between PDA pixels (left) and pump-probe delay times (right). 
signal from the detection elements within the relevant wavelength range (blue trace, the average of 56 pixels on our PDA). Similarly, we take the mean of sequential time points to obtain a representative spectrum for a given time range (red trace, the average from 56 spectra from 5.0-10.5 ps). Two phenomena are immediately apparent: The noise in Figure 3.2(a) is forming distinct vertical bands, and the blue kinetic trace suffers from considerably higher levels of noise than the red spectrum, even though they were generated from the same total number of samples.

Spectral and temporal correlations in $\Delta T / T$ noise can be quantified by taking the Pearson product-moment correlation coefficient, defining the detection elements as observations and time points as variables to measure spectral correlations (Figure 3.2(b), left) or vice versa to measure temporal correlations (Figure 3.2(b), right). We take our region of interest to be $650-760 \mathrm{~nm}$ (56 pixels) and $5.0-10.5 \mathrm{ps}$ (56 time points) as it contains a finite but spectrally and temporally flat signal, enabling us to assess noise from probe fluctuations, pump fluctuations, and shot readout, while avoiding correlations due to the dynamics of the absorption feature itself.

The correlation plots in Figure 3.2(b) show that there is a high degree of $\Delta T / T$ noise correlation between detection elements for data from a given set of time points, but almost no correlation between sequential time points for $\Delta T / T$ values from the same given set of detection elements. In other words, when averaging over 56 PDA pixels to generate the kinetic in Figure 3.2(a), our SNR does not improve as much as if those 56 pixels were independent measurements.

Incidentally, by conducting this analysis on data collected with unreferenced probe sources we are able to discern spectral regions of the probe light that are generated by different fundamental processes, due to discontinuities in their spectral correlation. For example, with the NOPA probe we see that light near $800 \mathrm{~nm}$ is anti-correlated with light from $570-790 \mathrm{~nm}$, as would be expected due to fluctuating conversion efficiency in the NOPA (see Figure 3.3).

\subsubsection{Chirp and effective sample number}

The maximum number of samples that we can collect within a given period of time $t$ is given by $N=\omega x t / 2$, where $\omega$ is the laser repetition rate, $x$ the number of detection elements (in our case, the 256 pixels on our PDA), and the factor of 


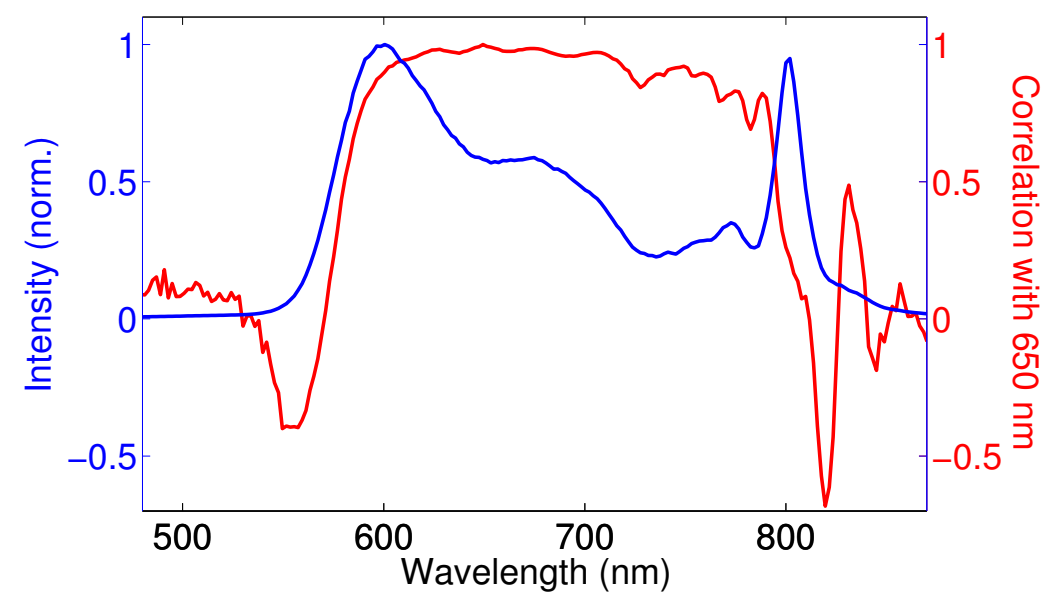

Figure 3.3: Unreferenced NOPA output intensity (blue) and correlation coefficient of $\Delta T / T$ at each wavelength with the value measured at $650 \mathrm{~nm}$. Correlations calculated from $\Delta T / T$ allow us to discriminate probe light originating from various fundamental processes. In this case, we can see that light at $800 \mathrm{~nm}$ (residual from the fundamental harmonic of our chirped-pulse amplifier) is anti-correlated with the amplified NOPA light, suggesting that a significant component of probe noise is due to fluctuations in the efficiency with which the NOPA is converting energy from $800 \mathrm{~nm}$ into the region from $580-790 \mathrm{~nm}$. 
$1 / 2$ since two laser shots are required to calculate a single $\Delta T / T$ value. However, as shown in Figure 3.2 our detection elements often do not make independent measurements, resulting in a reduced effective sample number.

The dispersion ('chirp') of ultrafast broadband laser pulses is typically considered problematic in ultrafast spectroscopy, with significant effort (such as the exclusive use of reflective optics) often taken to minimise this effect [113]. Nonetheless, some chirp (typically on the order of 1 ps over several hundred $\mathrm{nm}$ for a $100 \mathrm{fs}$ pulse, determined as shown below) is inevitably present in the probe and/or pump pulses at the sample, and a wavelength-dependent time offset is applied to correct the dataset. In some cases, this correction is sufficiently large that a single spectrum from the corrected dataset may be compiled from several shot pairs. One side effect of this correction is a reduction in noise correlation between detection elements for a single $\Delta T / T$ spectrum, and a corresponding increase in effective sample number given by $N=\omega x t / 2$. We sought to exploit this observation and increase our effective sample number by using highly chirped broadband probe pulses that span the signal of interest in both wavelength and time.

Figure 3.4(a) shows a dataset collected with the same sample and excitation conditions as in Figure 3.2 (small signal; low SNR), however the probe pulse has been strongly chirped by transmission through a $1 \mathrm{~cm}$ thick borosilicate window prior to interaction with the sample. The total chirp of the system is characterised by fitting a $3^{\text {rd }}$ degree polynomial to the coherent pump-probe artefact acquired during a high pump-power measurement. [113] The fitted polynomial (black dashed curve) determines the temporal offset applied to each wavelength to correct for the large amount of chirp, in this case totalling 12 ps from $660-760 \mathrm{~nm}$.

It is clear that the chirp correction transformation has 'rotated the bands of noise in Figure 3.4(b) away from the wavelength axis. The kinetic trace (blue) features significantly less noise than the equivalent trace in Figure 3.2, while there is some corresponding increase in noise in the spectrum (red). Figure 3.4(c) confirms that there is no longer any significant correlation between the pixels of our PDA, increasing the effective number of samples collected per laser shot pair.

\subsubsection{Quantification of sensitivity enhancement}

Each data point in the surface shown in Figure 3.4 is the average of 4,000 shot pairs, compared to 7,000 shot pairs per point in Figure 3.2. The reduced number of 

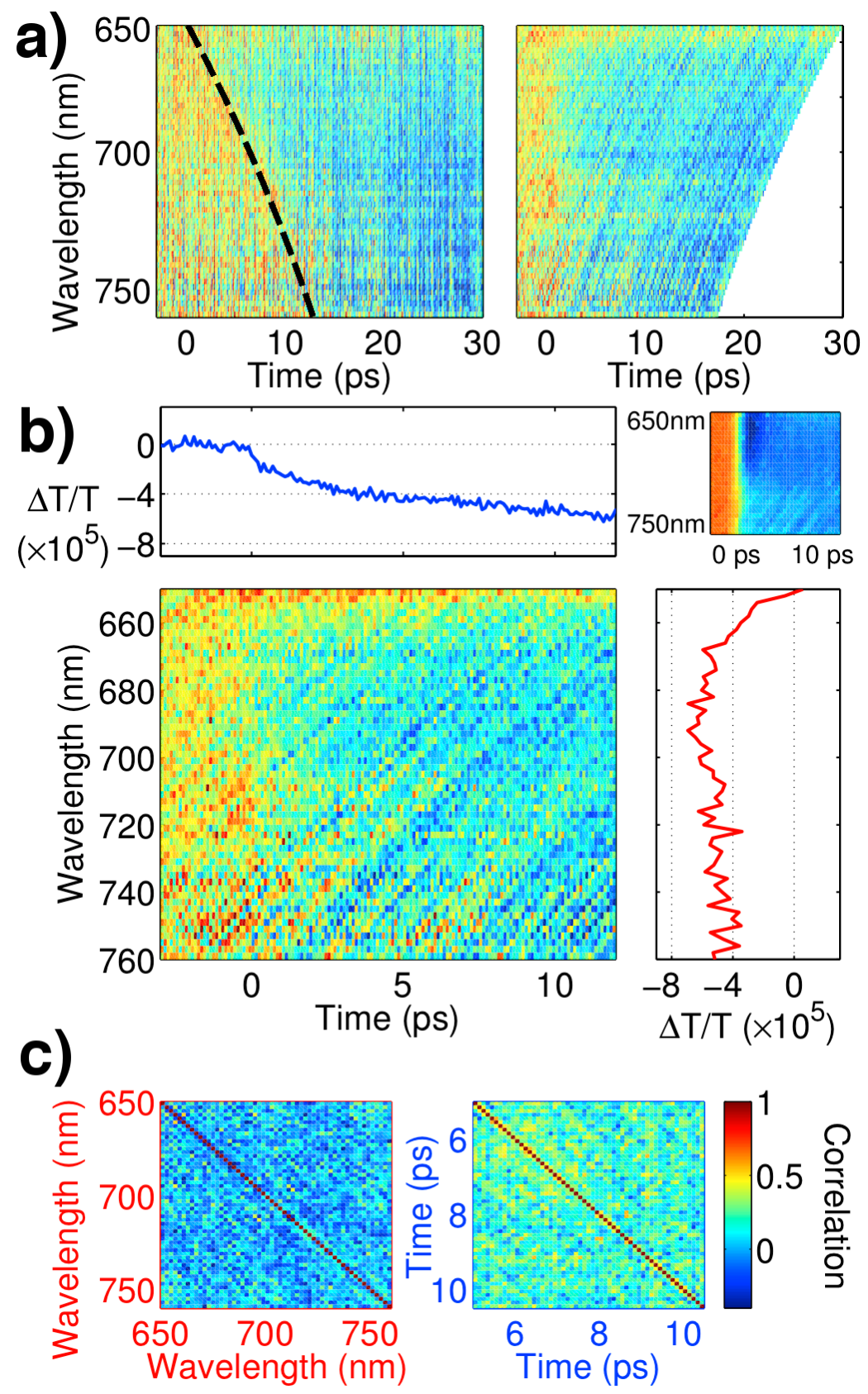

Figure 3.4: (a) P3HT:PCBM TA surface collected under weak excitation $\left(1 \mu \mathrm{J} \mathrm{cm}^{-2}\right)$ with a strongly chirped probe, raw data (left, with $3^{\text {rd }}$ order polynomial shown in black) and after chirp correction (right). (b) Corrected dataset with kinetics (blue), spectrum (red), and (insert) TA surface collected under strong $\left(71 \mu \mathrm{J} \mathrm{cm}{ }^{-2}\right)$ excitation. (c) Correlation between PDA pixels (left) and pump-probe delay times (right). 
shot pairs in the highly chirped measurement ensures that each dataset had exactly the same total acquisition time - 30 minutes to collect while operating our laser at a repetition rate of $3 \mathrm{kHz}$. This correction arises because using a highly chirped probe pulse results in the loss of measurements corresponding to pump-probe delay times that are moved out of the observation window by the chirp correction process (visible as data at times $>18 \mathrm{ps}$ in Figure 3.4(a)). The need to collect measurements at additional delay times reduces our effective data collection rate for highly chirped probes.

If the observed reduction of noise is fully explained by the effective sample number $N=\omega x t / 2$, we should see an improvement in sensitivity by a factor of $\sqrt{56 \times 4,000 / 7,000}=5.7$ when using 56 pixels on our PDA (assuming complete correlation between each pixel in the unchirped case). We assessed the noise by fitting a biexponential curve to the growth of the photoinduced absorption and taking the standard deviation of the residuals (Figure 3.5), finding that noise was in fact reduced by a factor of 5 , very close to the predicted value. To achieve this sensitivity with an unchirped pulse would have required data collection for 25 times longer, a collection time of over 12 hours. In most cases, this would be impractical to achieve due to slow drift (e.g., beam overlap, OPA output) and sample degradation. Here, we are able to cleanly resolve the exciton diffusion limited phase of charge photogeneration in a polymer:fullerene blend at an excitation density of only $1.3 \times 10^{17} \mathrm{~cm}^{-3}$, below the threshold for bimolecular exciton-exciton and exciton-charge annihilation reactions of approximately $10^{18} \mathrm{~cm}^{-3}$ [ref. [109]]. Alternatively, to achieve the same SNR with an unchirped probe by increasing the signal via excitation fluence would require a fivefold increase in pump power. This would risk operating in the non-linear regime, where the measured kinetic rates could be affected by bimolecular effects that are of no relevance to photovoltaic devices under solar illumination.

Fluctuations in pump pulse intensity contribute an additional noise component that is proportional to the signal amplitude. Unlike probe noise, pump fluctuations are not corrected by normalising between sequential shots, and therefore can introduce artifacts in the form of slow drift as well as fast fluctuation. Through the use of a highly chirped probe, measurements at many pump-probe delays are taken simultaneously rather than in sequential batches, and the spectrum for each time point comes from many pump shots. Slow pump noise (on the order of seconds-minutes) is therefore averaged out more effectively than by increasing 

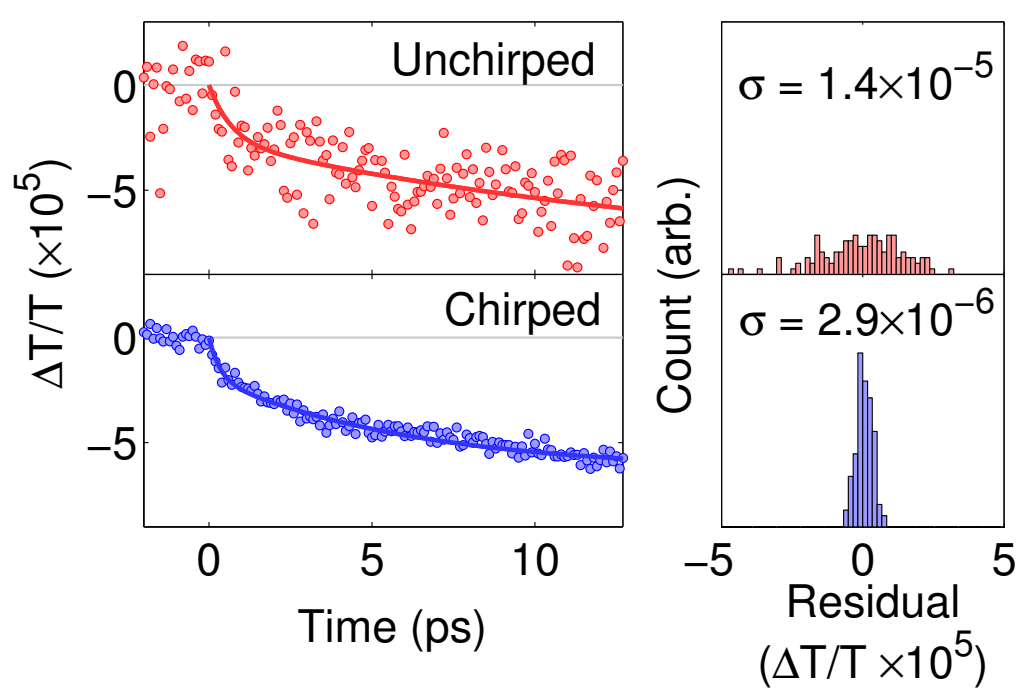

Figure 3.5: P3HT:PCBM photoinduced absorption with $100 \mathrm{fs}$ spaced time points. Kinetics were integrated from $660-760 \mathrm{~nm}$ with $<1$ ps probe chirp (red, 7, 000 shot pairs per point) and with $12 \mathrm{ps}$ probe chirp (blue, 4,000 shot pairs per point). Standard deviations represent deviation from biexponential fits. Each dataset was collected in approximately 30 minutes.

the sample number in the low-chirp case.

We also used the chirped probe method to investigate the photoswitching dynamics of photochromic [1,3] oxazine in acetonitrile solution ${ }^{\text {ii }}$. $[134,135]$ Figure 3.6(a) shows the TA surface collected with an unchirped probe pulse (unreferenced WLC generated in $\mathrm{CaF}_{2}$ window [113]) under strong pump excitation $\left(245 \mu \mathrm{J} \mathrm{cm}^{-2}\right.$ per pulse, $\left.300 \mathrm{~nm}\right)$. Two photoinduced absorption features are evident: a fast initial component centred near $500 \mathrm{~nm}$ that decays within $10 \mathrm{ps}$, and a higher energy absorption centred near $450 \mathrm{~nm}$ that grows over several tens of ps.

In order to assess the efficacy of our method under these conditions, TA data was collected under weak excitation $\left(14 \mu \mathrm{J} \mathrm{cm}^{-2}\right.$ per pulse), again with both unchirped and highly chirped probes. In the chirped case, probe light was transmitted through a $10 \mathrm{~cm}$ thick high-refractive-index glass block, dispersing the light by $67 \mathrm{ps}$ across the observation window of $400-530 \mathrm{~nm}$ before being sent through the sample and imaged onto 79 pixels of our PDA. The resulting traces in Figure 3.7 again show a significant improvement in SNR in the chirped case, to the extent 

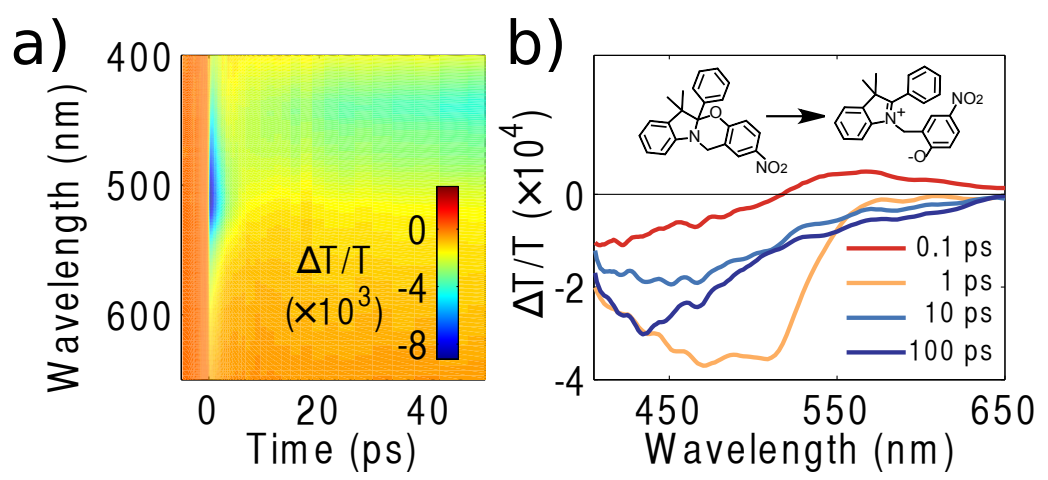

Figure 3.6: (a) TA surface of the [1,3] oxazine shown in (b) collected under strong excitation $\left(245 \mu \mathrm{J} \mathrm{cm}^{-2}, 300 \mathrm{~nm}\right)$ and minimal chirp (b) [1,3] Oxazine structure and TA spectra collected under weak excitation $\left(14 \mu \mathrm{J} \mathrm{cm}^{-2}, 300 \mathrm{~nm}\right)$ with an unchirped probe at 4 discrete time points. 25, 000 shot pairs per spectra, 6 minute total collection time.

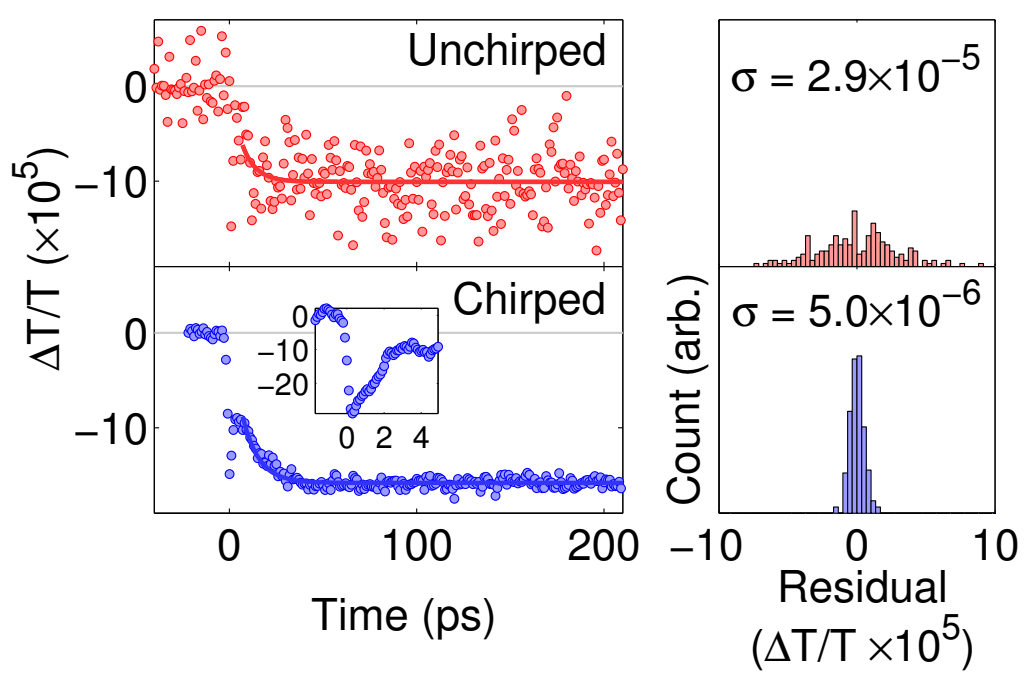

Figure 3.7: Kinetics of the photoinduced absorption in oxazine in acetonitrile solution under weak excitation $\left(14 \mu \mathrm{J} \mathrm{cm}^{-2}, 300 \mathrm{~nm}\right)$ from $400-530 \mathrm{~nm}$ with $<1$ ps probe chirp (red, 6, 000 shot pairs per point) and with $67 \mathrm{ps}$ probe chirp (blue, 3,000 probe pairs per point). Standard deviations represent deviation from monoexponential fits at pump-probe delay $>4$ ps. Each dataset was collected in approximately 60 minutes. 
where the chirped case reveals the fast initial absorption feature that would likely have been mistaken for noise in the unchirped case. Monoexponential fits at delay times beyond 4 ps (to avoid the faster absorption signal) reveal sensitivity was improved by a factor of 5.8, in excellent agreement with the expected value of 6.3. The confidence bounds of the fitted time constants emphasize the value of the improved sensitivity for acquiring small signals: $\tau=7.3$ ps $(4.9-14.1 \mathrm{~nm}, 95 \%$ confidence) for the unchirped data and $\tau=8.7 \mathrm{ps}(8.2-9.2 \mathrm{~nm}, 95 \%$ confidence) for the chirped data.

As seen in Figure 3.4, one side effect of highly chirped probe pulses is a loss of SNR in collected spectra because chirp effectively rotates spectra to cut across the bands of noise. Our solution is to collect data for spectra with an unchirped probe, separately from kinetics. In this way, a handful of spectra can be collected with extremely high sample number at selected values of pump-probe delay. When combined with the kinetics dataset, we obtain a complete picture of the photodynamics under weak excitation.

Each spectrum in Figure 3.6(b) is the average of 25, 000 individual $\Delta T / T$ spectra, with the dataset ( 5 time points, including one at negative delay time for background correction) taking 6 minutes in total to collect. We can see from the spectra the loss of the initial signal by $10 \mathrm{ps}$, and presence of the higher energy absorption at 100 ps. The spectra clearly capture the features seen in the surface in Figure 3.6(a), but with nearly $20 \times$ lower excitation fluence. Although irreversible photochemistry is not observed in this case, the ability to measure at substantially lower fluences is essential for samples that accumulate photoproduct proportionately to the number of absorbed photons in each shot.

\subsection{A recipe for instant, easy improvement in sensi- tivity}

\subsubsection{Limits of detection; optimization}

Assuming complete correlation between detection elements for an unchirped probe pulse, the sensitivity of our experiment with a chirped probe dispersed over 
$x$ detection elements (pixels) is given by

$$
\left(\frac{\Delta T}{T}\right)_{\mathrm{RMS}, \mathrm{n} \text { shot pairs }}=\frac{\left(\frac{\Delta T}{T}\right)_{\mathrm{RMS}, 1 \text { shot pair }}}{\sqrt{n \cdot E F}}
$$

where the enhancement factor

$$
\begin{gathered}
E F=\frac{\operatorname{chirp}(\mathrm{ps})}{t \text { spacing }(\mathrm{ps})} \\
1 \leq E F \leq x \& \text { number of } t \text { points. }
\end{gathered}
$$

Here, we approximate dispersion as being linear with wavelength, and refer to chirp as the total time spanned by the chirped broadband pulse. The enhancement to sensitivity achieved through the use of a chirped probe is limited by either the number of detection elements or the number of pump-probe delay values, whichever is smaller. In fact, the optimum amount of chirp for a given experiment is the minimum chirp that results in each pixel taking its measurement of $\Delta T / T$ at a given (corrected) delay time from a different shot pair. In other words,

$$
\text { optimum chirp }(\mathrm{ps})=x(t \text { spacing }(\mathrm{ps}))
$$

as the probe is chirped beyond the optimum point, sensitivity decreases again as measurements must be made at additional time points prior to application of the chirp correction algorithm, reducing the time available for data collection at relevant time points.

Figure 3.8 shows calculated sensitivity using a probe with the inherent noise equal to the referenced NOPA data given in Figure 3.1, taking into account loss of shots due to the chirp correction algorithm. Sensitivity at the optimum point approaches the limit determined by the detector, in this case $(\Delta T / T)_{\mathrm{RMS}}=1.8 \times 10^{-6}$ for 10, 000 shot pairs collected at 256 time points, which can be acquired in under 30 minutes at a repetition rate of $3 \mathrm{kHz}$. Without applying chirp, achieving this sensitivity would take an impractically long 5 days of continuous data collection. We note that this sensitivity does not represent an absolute limit, even for a 256 element detector array, because we took a $(\Delta T / T)_{\mathrm{RMS}}$ inherent to a non-optimized shot pair as the starting point. Any additional improvements to the inherent $\Delta T / T$ sensitivity, including improving the probe stability, implementing shot rejection, or operating at higher repetition rates, could be augmented with our highly chirped probe method in order to achieve exceptional sensitivity. 


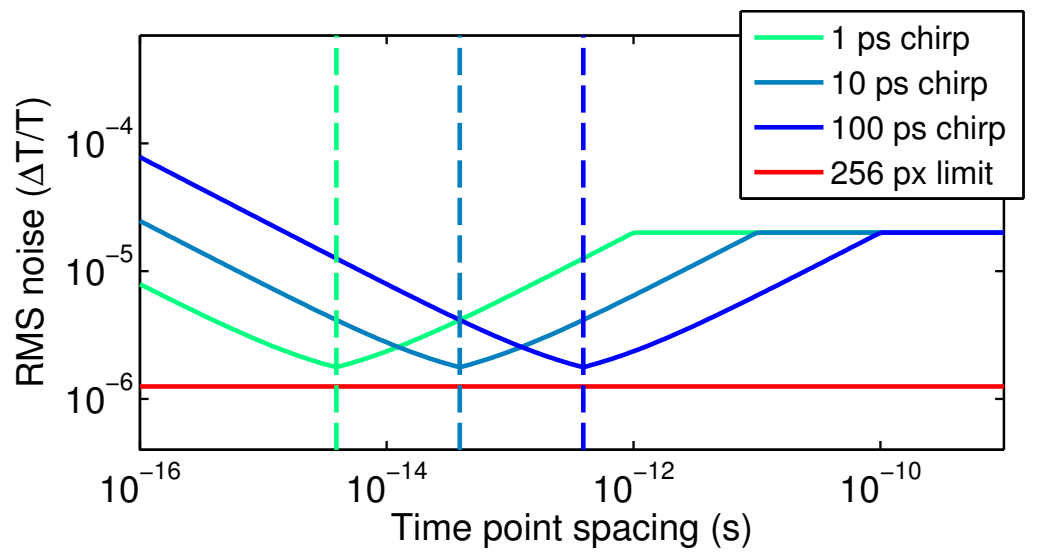

Figure 3.8: Calculated sensitivity assuming 210-3 sensitivity for a single shot pair (referenced NOPA) with 256 time points, linearly spaced. The simulation assumes 10,000 shot pairs for the unchirped case, with a reduction in shot number as chirp is increased (responsible for increasing noise at low ). Dispersion is approximated as being linear with wavelength. Dashed lines represent time resolution degradation under each value of probe chirp.

\subsubsection{Reduction of IRF due to chirp}

While it has been shown that the use of strongly chirped probe pulses does not necessarily degrade the temporal resolution of a transient absorption experiment, [136] in our highly chirped experimental configuration there is some degradation due to the probe chirp across the wavelength range subtended by each individual detector element. In other words,

$$
\operatorname{IRF} \text { degradation }(\mathrm{ps})=\frac{\operatorname{chirp}(\mathrm{ps})}{x},
$$

such that when the probe is optimally chirped for a given time point spacing, each pixel is monitoring a unique time point, and the spacing of those time points represents the limit to the temporal resolution of that experiment.

The set of pump-probe delays selected for a given experiment must have sufficiently small spacing so as to capture the fastest of the dynamics under investigation, ideally distributing the total number of data points (256 in the case of an optimally chirped probe with a 256 element detector) over $\sim 3$ to $5 \tau$, where $\tau$ is the lifetime of interest. The optimum amount of chirp for high sensitivity kinetics therefore also results in an amount of temporal degradation that is substantially $(>50 \times)$ less than the timescales under investigation. 


\subsection{Experimental details}

Regio-regular poly(3-hexyl-thiophene-2, 5-diyl) (P3HT) was purchased from American Dye Source, Inc (ADS306PT). [6,6]-Phenyl $\mathrm{C}_{61}$ butyric acid methyl ester (PCBM) was purchased from Sigma-Aldrich. A $1: 1$ blend of P3HT:PCBM was dissolved in chlorobenzene $\left(30 \mathrm{mg} \mathrm{ml}^{-1}\right)$ and spin-coated onto a quartz substrate to produce a $90 \mathrm{~nm}$ thick layer before being vapour annealed for 30 minutes in a jar containing 1,2-dichlorobenene. TA spectroscopy of P3HT:PCBM was conducted under dynamic vacuum at room temperature.

The $[1,3]$ oxazine was synthesized according to reference [135] and will be the subject of a separate publication. The oxazine was dissolved in acetonitrile solution at $0.5 \mathrm{moll}^{-1}$ in a $200 \mu \mathrm{m}$ path length quartz cuvette, spectroscopy conducted at room temperature and pressure. Broadband light was generated in $\mathrm{CaF}_{2}$ window that was slowly translated in order avoid photodamage. [113]

\subsection{Conclusions}

We assessed the noise characteristics of several commonly used ultrafast TA probes, showing that noise from probe fluctuations and shot readout is often strongly wavelength correlated. We introduced an effective, and widely applicable method to decorrelate this noise, capable of improving sensitivity tenfold simply through the insertion of a glass block in the beam path to strongly chirp the probe pulse. This method was demonstrated by resolving exciton diffusion limited charge generation in organic photovoltaic cells under solar equivalent excitation density, and by investigating molecular photoswitching under low excitation fluence. A scheme was presented to enable application and optimization of this technique in TA experiments, although it could equally be applied to broadband ultrafast photoluminescence spectroscopy. [98,137,138] 


\title{
Chapter 4
}

\section{Charge recombination in polymer:fullerene blends}

\begin{abstract}
Recent work indicates that the process of free charge generation in OPVs is mediated by processes on ultrafast timescales that imply a branching of photogenerated charges into bound and well separated populations. Density dependent measurements of recombination are able to distinguish these populations. We measure the free charge yield in a range of charge generating blends of various efficiency, assembling a library of samples that will be further exploited in the following chapter.

Additionally, we discuss the connection between electronic and optical measurements of charge density, and show how they can be reconciled to allow measurement of charge recombination over an exceptional range of timescales $\left(10^{-13}-10^{-2} \mathrm{~s}\right)$ and charge densities $\left(10^{13}-10^{19} \mathrm{~cm}^{-3}\right.$ and above).
\end{abstract}

\subsection{Introduction}

While the processes allowing physical separation of tightly-bound charge pairs in OPVs is still not fully understood, much progress has been made recently towards understanding the role of charge-transfer (CT) states. In particular, as discussed in Section 1.3.3, there is growing evidence that relaxed CT states represent a loss channel rather than an intermediate state in the process of free charge generation, with the charge pairs that dissociate completely doing so via the manifold of 
excited CT states. $[96,99,100]$ A prominent aspect of such a system would be, upon transient excitation, a branching of excited states into 'bound' and 'free' charge pairs on the timescale of CT state thermalization. OPV blends that do not exhibit a favourable branching ratio are therefore likely to exhibit low photocurrent yields, even if they are highly efficient at the initial exciton quenching/charge-transfer step.

Reliably measuring the yield $\Phi_{\text {free }}$ of free charges and its dependence on crucial device parameters such as polymer materials or morphology is an important step towards determining how to design materials that encourage highly efficient charge separation at a minimal energetic cost. However, reliably measuring $\Phi_{\text {free }}$ is not a trivial task.

To determine $\Phi_{\text {free }}$ electronically in functioning OPV devices, measurements of device photocurrent must take into account not only the fraction of free charges, but the competition of charge extraction with charge recombination, which itself (in the case of non-geminate/bimolecular recombination) exhibits a quadratic dependence on charge carrier density. Any losses due to poor extraction pathways or interfaces at the electrode are further convoluted with the initial branching step, obscuring the fundamental physics of charge separation.

A significant amount of work has been published investigating charge recombination by electronic methods such as time-of-flight [139] and photo induced charge extraction by linearly increasing voltage (photo-CELIV), [140]. Work by Shuttle et al. has sought to make the connection between macroscopic electronic measurements and the dependence on charge density of bimolecular recombination rate, [141-143] charge mobility, [144] and open-circuit voltage. [145]

Time-resolved electronic measurements are limited to time scales of nanoseconds or longer, several orders of magnitude slower than ultrafast optical measurements. However, as electronic measurements are able to resolve much smaller charge densities than optical measurements, combining the two techniques will enable measurements of the full charge photogeneration-recombination process, from femtosecond to millisecond timescales, over a huge range of charge densities. In Section 4.6 we address discrepancies that exist between spectroscopic and electronic measurements of recombination in OPVs, and show that they can be reconciled through appropriate handling of the data. The result is a picture of charge recombination spanning an unprecedented range of timescales and charge densities. 
Direct spectroscopic measurements of parameters such as free charge yield or absolute charge carrier density face their own set of challenges. The spectral signatures of polaron absorption from bound and free charge pairs is typically overlapping, if not completely indistinguishable, and charge pairs generally recombine non-radiatively (preventing the use of photoluminescence techniques).

One approach, demonstrated by Howard et al., [102] is to take advantage of the difference in mobility of the bound and free charge pairs. Charges in a bound interfacial state are immobile and recombine geminately, a process which is both rapid (typically within $\sim 2 \mathrm{~ns}$ ) and monomolecular. Contrastingly, free charges will (in a spectroscopic film, where extraction at the electrode is not possible) typically undergo non-geminate Langevin recombination with an opposite charge that originated from a separate absorption event. This process is bimolecular, occurring with a rate that is nonlinear with respect to charge density (and therefore initial excitation density). It is thus clear how measurements of the density dependence of recombination - even though they may be a relatively 'slow' measurement in terms of instrument response - can provide insight into the primary photogeneration process occuring within the first $100 \mathrm{fs}$ of the photogeneration process.

We discuss the use of the density-dependent measurements of recombination in more detail and implement it in Section 4.5 below. We investigate a range of blends of varying efficiency, and correlate their efficiency to measured recombination parameters. While this work is based on established techniques, it serves to build a library of well characterised materials that we will then take advantage of through the introduction of a new technique in Section 5.

\subsection{Experimental: Sample preparation}

All materials were commercially sourced, from the following suppliers:

- $\mathrm{PC}_{61} \mathrm{BM}$ Aldrich.

- P3HT Aldrich. Average MW 30-60 kDa

- PCDTBT Solaris Chem Inc. Typical MW 20-45 kDa

- PCPDTBT Solaris Chem Inc. Typical MW 15-25 kDa 
- MEH-PPV American Dye Source, Inc. MW > 100 kDa

Samples were spin-coated onto spectrosil fused silica substrates in air and at room temperature. The substrates were cleaned prior to coating by sonication in acetone and then in isopropanol. Each sample was prepared from solution as follows:

- P3HT:PC ${ }_{61} \mathrm{BM}$ (1:1, and 19:1), $30 \mathrm{mg} / \mathrm{mL}$ total concentration in 1,2-dichlorobenzene. Spin coated at 2000 RPM. Where indicated, the film was annealed by exposure to a solvent (1,2-dichlorobenzene) vapor saturated atmosphere at room temperature for 30 minutes. [146]

- PCPDTBT:PC ${ }_{61} \mathrm{BM}$ (1:2), $30 \mathrm{mg} / \mathrm{mL}$ total concentration in chlorobenzene, with 5\% 1,8-octanedithiol. [101] Spin coated at 1200 RPM.

- PCDTBT:PC ${ }_{61} \mathrm{BM}(1: 4), 30 \mathrm{mg} / \mathrm{mL}$ total concentration in 1,2-dichlorobenzene. Spin coated at 1500 RPM.

- PCDTBT:PC ${ }_{61} \mathrm{BM}$ (19:1), $7 \mathrm{mg} / \mathrm{mL}$ total concentration in 1,2-dichlorobenzene. Spin coated at 2000 RPM.

- MEH-PPV:PC ${ }_{61} \mathrm{BM}$ (1:1), $15 \mathrm{mg} / \mathrm{mL}$ total concentration in chlorobenzene. Spin coated at 1700 RPM.

- MEH-PPV:PC ${ }_{61}$ BM (1:4), $15 \mathrm{mg} / \mathrm{mL}$ total concentration in chlorobenzene. Spin coated at 1200 RPM.

\subsection{Broadband TA spectra}

The four polymers studied here (MEH-PPV, P3HT, PCDTBT and PCPDTBT) form charge generating bulk heterojunction blends with the electron acceptor PCBM, with reported power conversion efficiencies ranging from $\approx 0.5 \%$ for MEH-PPV:PCBM [147] to > 6\% for PCPDTBT:PCBM. [148] Furthermore, the photogenerated charge yield and fill factor of these blends can be controlled by adjusting the polymer:fullerene ratio, by thermal or vapour annealing, or by the addition of a cosolvent to the solution prior to spin coating. Finally, differences in performance may be observed by selectively exciting either polymer or fullerene domains through careful selection of excitation wavelength. This section serves to 
characterize the free charge yield in a selection of OPV blends that use the above parameters to exhibit a range of photocurrent efficiencies.

Before undertaking recombination measurements, we collected ultrafast broadband visible TA of the blends in order to determine the origin of their excited state signatures. Broadband TA spectra of the samples were collected using an amplified broadband probe source (NOPA, reference [116]) and $100 \mathrm{fs}$ excitation at $532 \mathrm{~nm}$, generated in a parametric amplifier (TOPAS). As with the anisotropy measurements, pump-probe delay was varied using a retroreflector mounted on a mechanically controlled delay stage. The probe light was dispersed, collected and read-out at $3 \mathrm{kHz}$ using the spectrometer and photodiode array described in the main text.

The spectra shown below were taken at room temperature, and (in the charge generating blends) low excitation density (at or below $10 \mu \mathrm{J} \mathrm{cm}^{-2}$ pulse $^{-1}$ ) to avoid artifacts from higher order processes such as exciton-exciton and exciton-charge annihilation. For the sake of faster measurements, the neat blends were collected at higher excitation density as the goal was only to identify spectral features (rather than measure any rates). Nearly all samples show a broad, long-lived photoinduced absorption at $800 \mathrm{~nm}$ (the probing wavelength for the intensity-dependent recombination measurements and also for the measurements introduced in Chapter 5). The only exception is PCPDTBT:PCBM, for which $800 \mathrm{~nm}$ is coincident with the GSB.

Details regarding sample fabrication are included at the end of this chapter in Section 4.2.

\subsubsection{P3HT}

Poly(3-hexylthiophene-2,5-diyl) (P3HT), particularly when paired with PCBM, is well known as the benchmark system for OPV research, with an enormous number of published articles over the last decade and reported power conversion efficiencies of up to $4.5 \%$. [68] At least part of this popularity is due to the favourable and easily controllable crystallization that takes place between the two materials.

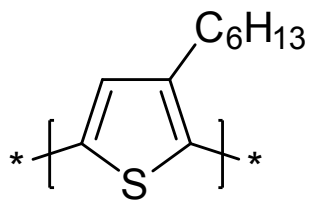

Figure 4.1: P3HT structure. 


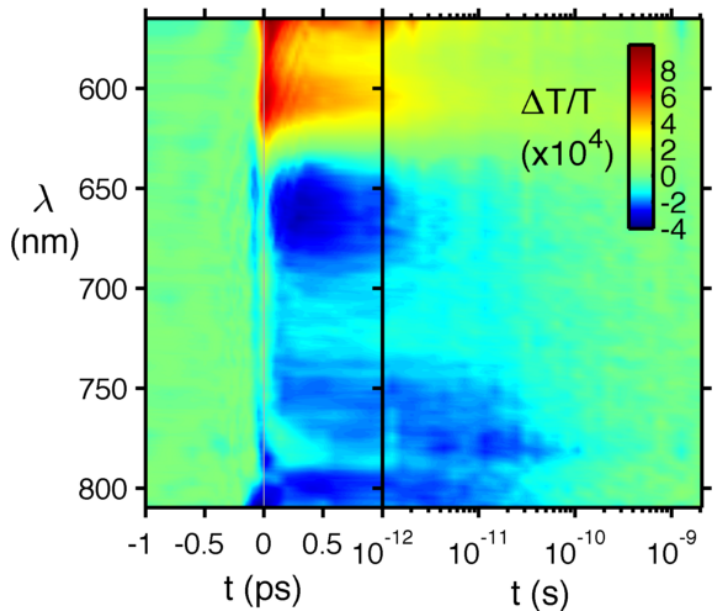

(a)

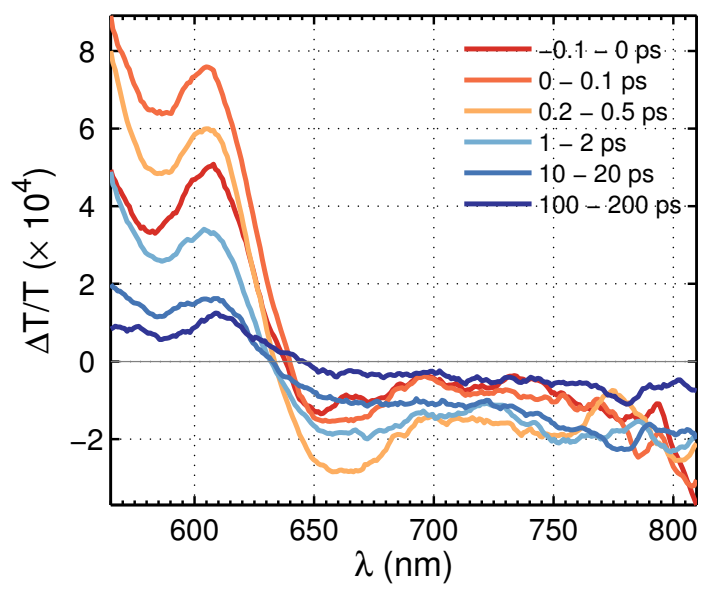

(b)

Figure 4.2: TA map (a) and spectra (b) of a neat P3HT film.

Figure 4.2 shows TA of a neat film of P3HT. The positive signal below $630 \mathrm{~nm}$ is due to ground-state bleach (GSB), where the excitation of chromophores from the ground state reduces total absorption at those wavelengths, resulting in an increase in differential transmission $(\Delta T / T)$. The vibronic structure in the GSB implies a significant degree of order in the film. The photoinduced absorption (PIA) visible near $650 \mathrm{~nm}$ and $800 \mathrm{~nm}$ suggests a broad PIA overlapping with stimulated emission (SE) in the region from $680-780 \mathrm{~nm}$. These signals will be primarily due to singlet excitons, with some contribution from bound interchain charges generated as described in references [125] and [126].

The addition of PCBM to P3HT results in a drastic change to the TA signal, with data from the as-cast (i.e. not annealed) film shown in Figure 4.3. The most obvious feature is the emergence of a large PIA that overlaps with the GSB, spanning from $\sim 620 \mathrm{~nm}$ to the NIR and persisting for much longer than the excitonic signal in the neat polymer film. The GSB shows reduced structure indicating significantly amorphous blend, and the SE appears to be entirely quenched.

The trace in Figure 4.4 shows the kinetics of the unannealed blend probed at $800 \mathrm{~nm}$, with combined datasets using a $100 \mathrm{fs}$ pump pulse and mechanical delay stage $\left(10^{-13}-10^{-9} \mathrm{~s}\right)$ and Q-switched, 700 ps pump and electronic delay stage $\left(10^{-9}\right.$ $10^{-4} \mathrm{~s}$ ). From the spectra we can attribute this signal entirely to polaron absorption, since the excitonic SE is completely absent in the blended film, indicating highly efficient charge transfer in to the PCBM domains. The TA amplitude can therefore 


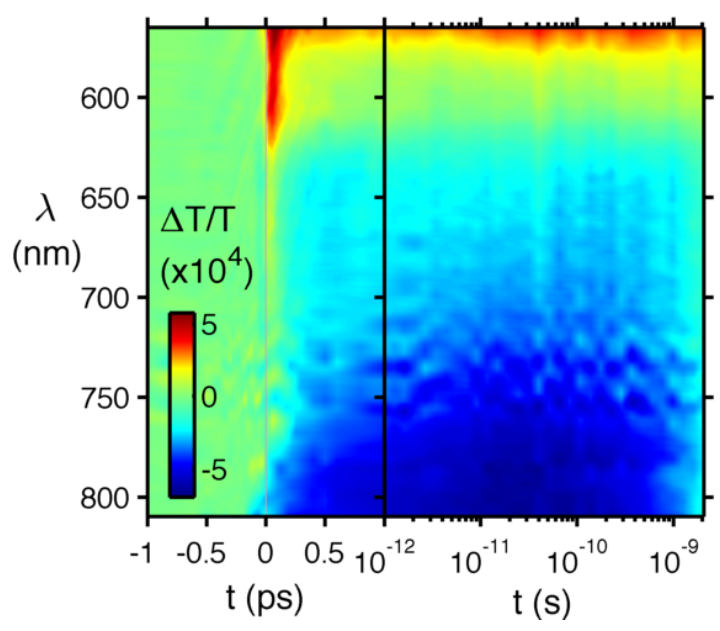

(a)

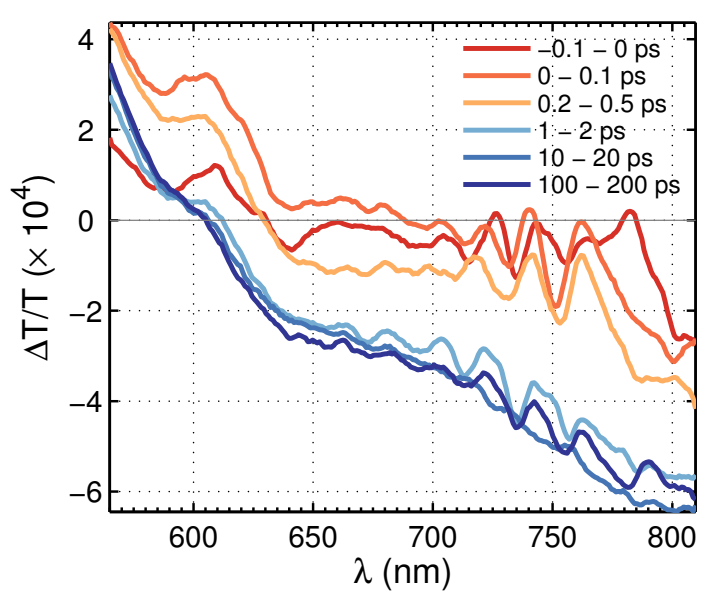

(b)

Figure 4.3: TA map (a) and spectra (b) of P3HT:PCBM (1:1) film, as-cast.

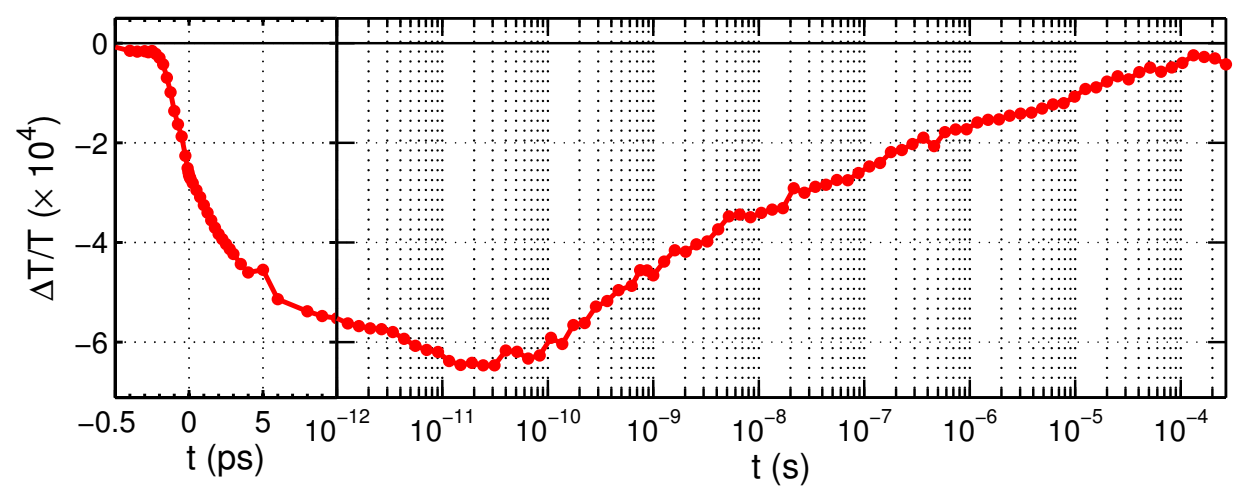

Figure 4.4: Combined fs and ps TA of P3HT:PCBM (1:1) film, as-cast, probed at $800 \mathrm{~nm}$. 
be assumed to be proportional to total photogenerated charge density. Most noticably we see that the majority of charges are formed within $\sim 300 \mathrm{fs}$, with some residual growth over the following $10 \mathrm{ps}$ due to diffusion of excitons from the small fraction of crystalline polymer domains.

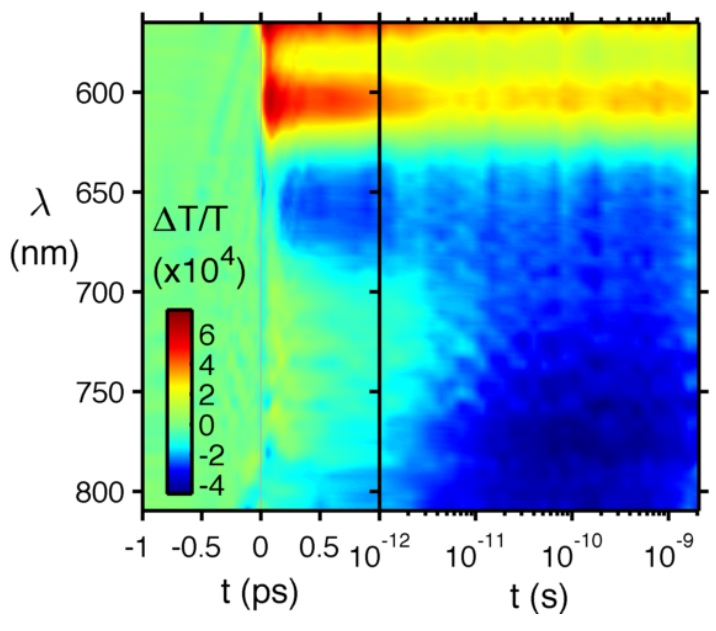

(a)

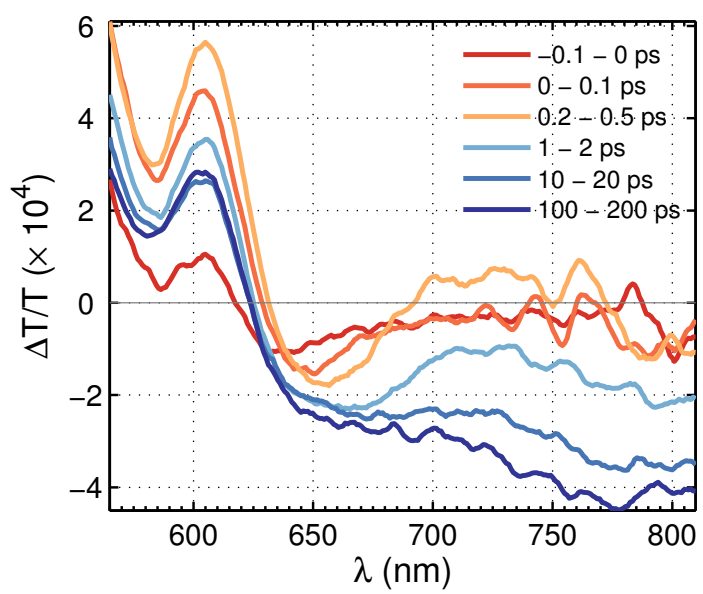

(b)

Figure 4.5: TA map (a) and spectra (b) of P3HT:PCBM (1:1) film, annealed.

Upon annealing (Figure 4.5) we see a reemergence of the vibronic GSB structure, indicating greater crystalline order within the polymer domains. The long-lived polaron PIA from the unannealed blend is still present, but overlapping with excitonic SE, indicating that not all excitons are initially quenched.

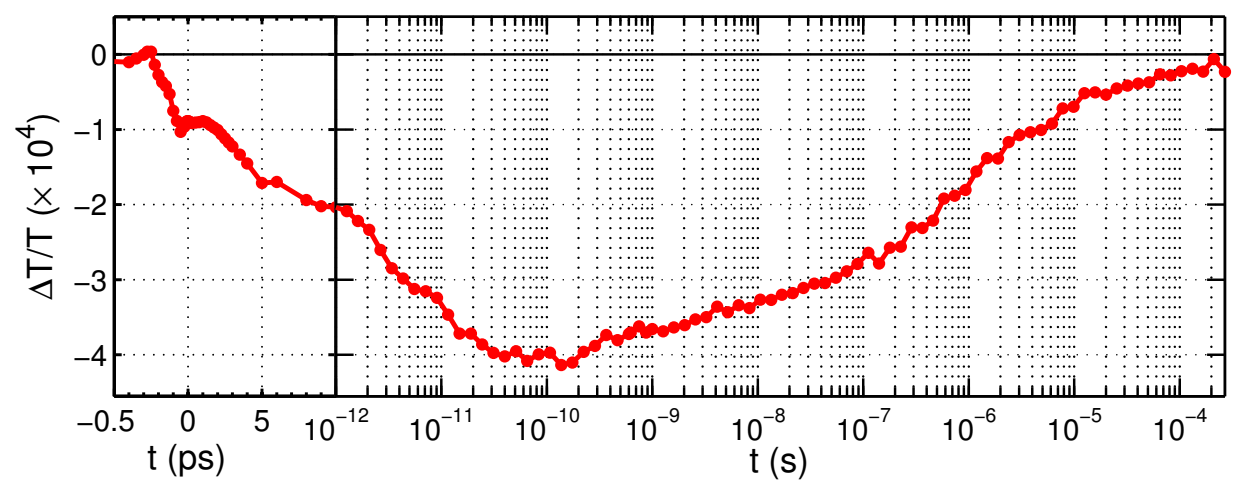

Figure 4.6: Combined fs and ps TA of P3HT:PCBM (1:1) film, annealed, probed at $800 \mathrm{~nm}$.

The kinetics at $800 \mathrm{~nm}$ for the annealed blend (Figure 4.6) are now affected 
by the overlapping exciton SE and polaron PIA, with a much slower rise in the signal due to the larger fraction of excitons that must diffuse from the crystalline polymer bulk to the P3HT:PCBM interface before being quenched. However, on the timescales measured with the Q-switched excitation source $\left(>10^{-9} \mathrm{~s}\right)$, the exciton SE is gone and the signal amplitude is again proportional to total photoinduced charge density.

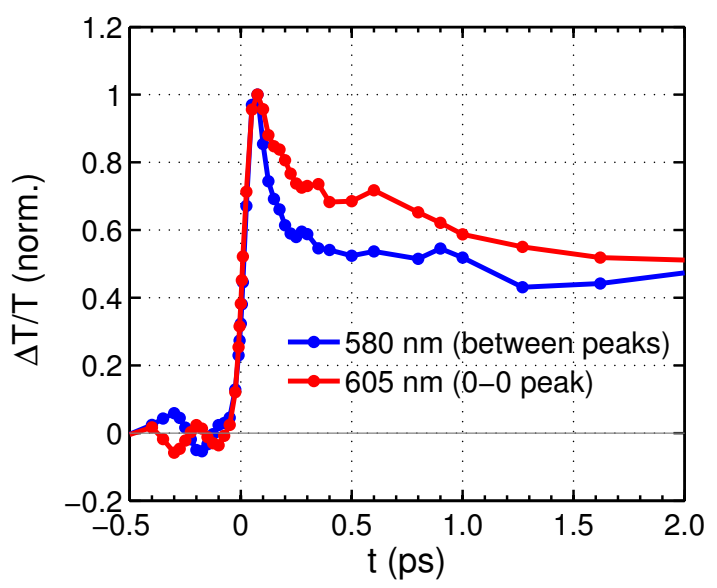

Figure 4.7: Kinetic traces from Figure 4.5a at two wavelengths. Early vibronic relaxation indicates that excitons in disordered polymer regions are rapidly either quenched by PCBM or energetically driven to more ordered regions.

It is interesting to note that, at very early times in the annealed blend, the spectra resemble the amorphous unannealed blend. As shown in Figure 4.7, the degree of vibronic structure shown in the GSB increases over $\sim 300 \mathrm{fs}$ following photoexcitation, indicating either ultrafast quenching of excitons in disordered regions of the polymer, or rapid downhill intrachain energy transfer to more ordered conformational subunits.

It is worth noting that the change in energetics induced by morphological changes such as annealing can result in a spectral shift in ground state absorption. As a result, the choice of excitation wavelength can influence the observed phenomena by selectively exciting morphological subpopulations of the sample. Figure 4.8 shows the TA spectra of both the annealed and as-cast P3HT:PCBM films, with excitation now at $570 \mathrm{~nm}$. In contrast to the data shown in Figures $4.3 \mathrm{~b}$ and $4.5 \mathrm{~b}$, these spectra seem to show no change in excited state behaviour upon annealing. It turns out that the low energy $570 \mathrm{~nm}$ pump is only significantly absorbed in the crystalline polymer domains, resulting in selective probing of 


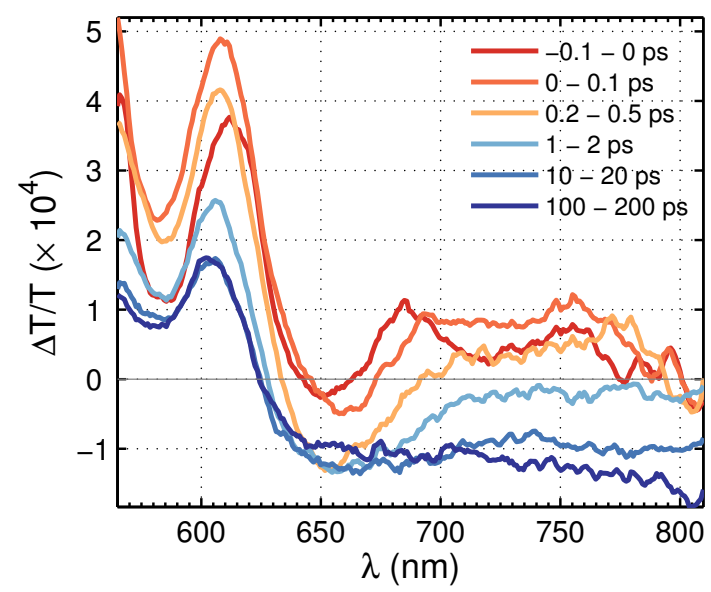

(a) P3HT:PCBM, annealed

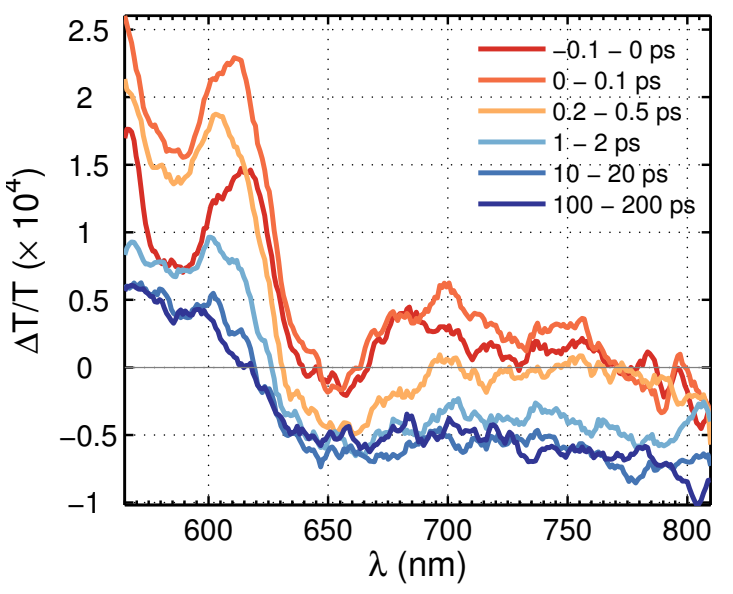

(b) P3HT:PCBM, as-cast

Figure 4.8: The choice of excitation wavelength can influence the observed phenomena by selectively exciting morphological subpopulations of the sample. In this case the samples are pumped at $570 \mathrm{~nm}$, selectively exciting the ordered regions of the polymer. In contrast to the previous data (pumped at $480 \mathrm{~nm}$ ), the samples now seem to exhibit idential excited state behaviour.

those regions of an otherwise amorphous film. This explains the lower signal amplitude in the unannealed spectra, and highlights the importance of consideration of excitation energies when comparing the photophysics of several samples.

\subsubsection{PCDTBT}

Poly[N-9-heptadecanyl-2,7-carbazole-alt5,5-(4,7-di-2-thienyl-2,1,3- benzothiadiazole)], otherwise known as PCDTBT, is a newer generation donor-acceptor copolymer. PCDTBT has been used (with $\mathrm{PC}_{71} \mathrm{BM}$ ) to make extremely efficient devices with over $6 \%$ power conversion efficiency, which it achieves despite the fact that it does not feature as

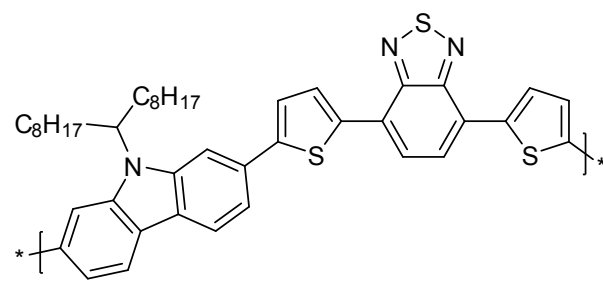

Figure 4.9: PCDTBT structure. low a bandgap as similar materials such as PCPDTBT. This impressive performance can be attributed to extremely efficient free charge photogeneration, with 
internal quantum yield (the ratio of extracted charges in a device to absorbed photons) shown to approach $100 \%$ [149].

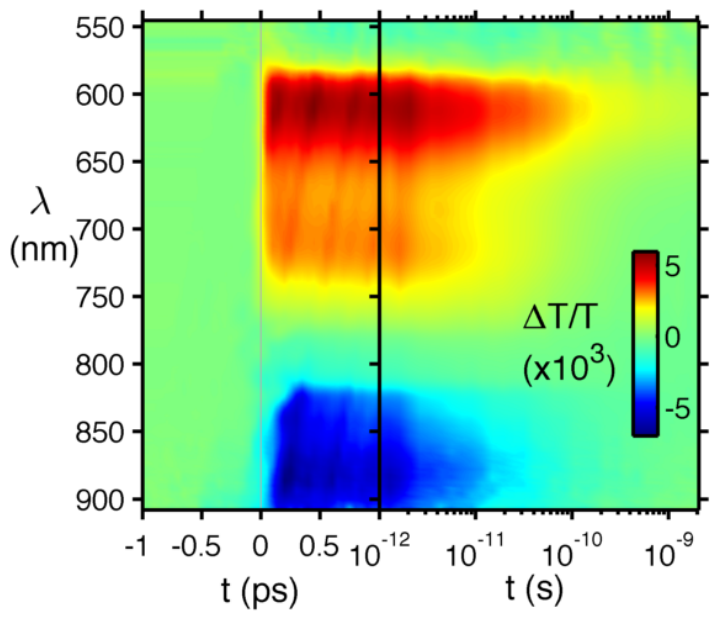

(a)

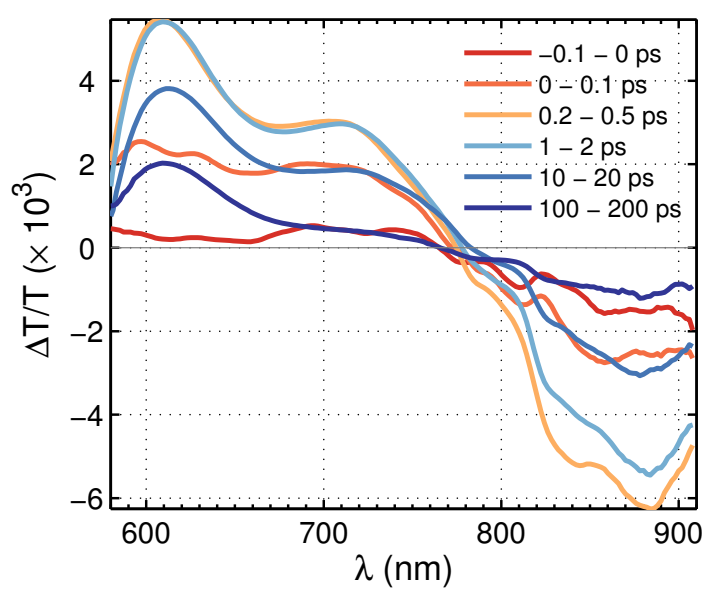

(b)

Figure 4.10: TA map (a) and spectra (b) of neat PCDTBT film.

TA of neat PCDTBT films, shown in Figure 4.10, show strong GSB centered at $610 \mathrm{~nm}$, overlapping with excitonic SE extending to $780 \mathrm{~nm}$ (which we assign based on its absence in the presence of PCBM and by comparison to steady state UV-vis and fluorescence spectroscopy [150]) and a PIA reaching from $810 \mathrm{~nm}$ into the NIR.

Upon blending with PCBM in an optimized 1:4 ratio, [100,149] we see similar effects reminiscent of P3HT:PCBM, with highly efficient quenching of SE, and a broad long lived PIA attributed to photogenerated polaron absorption. Some spectral shifting of the GSB can be attributed to charge migration to lower energy sites over $10-100$ ps timescales.

The kinetics of PCDTBT:PCBM at $800 \mathrm{~nm}$ show that nearly all charges are formed within several hundred femtoseconds, with very little residual growth consistent with reports of reduced crystallinity in PCDTBT compared to P3HT. [150] Again, our signal amplitude when probed at $800 \mathrm{~nm}$ is proportional to photoinduced charge density. 


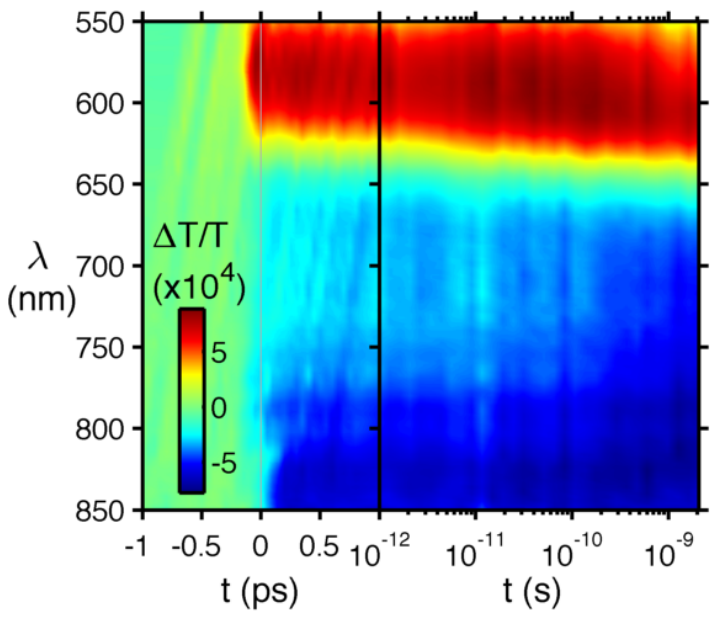

(a)

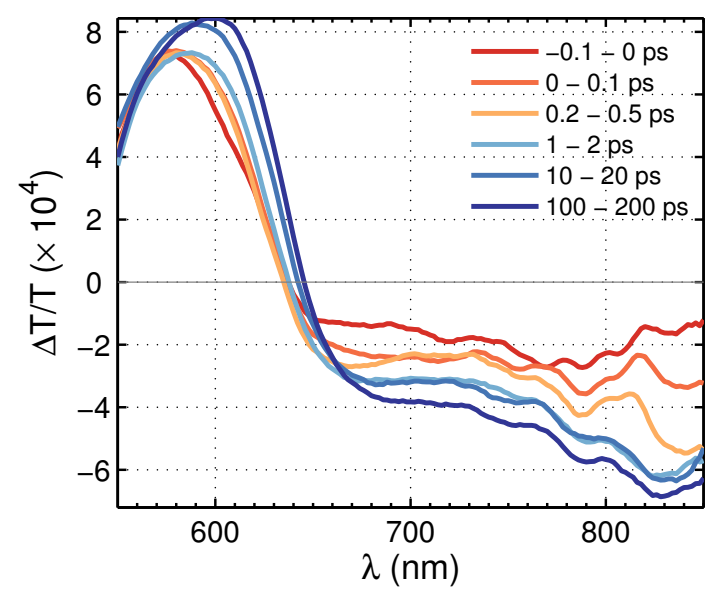

(b)

Figure 4.11: TA map (a) and spectra (b) of PCDTBT:PCBM (1:4) film.

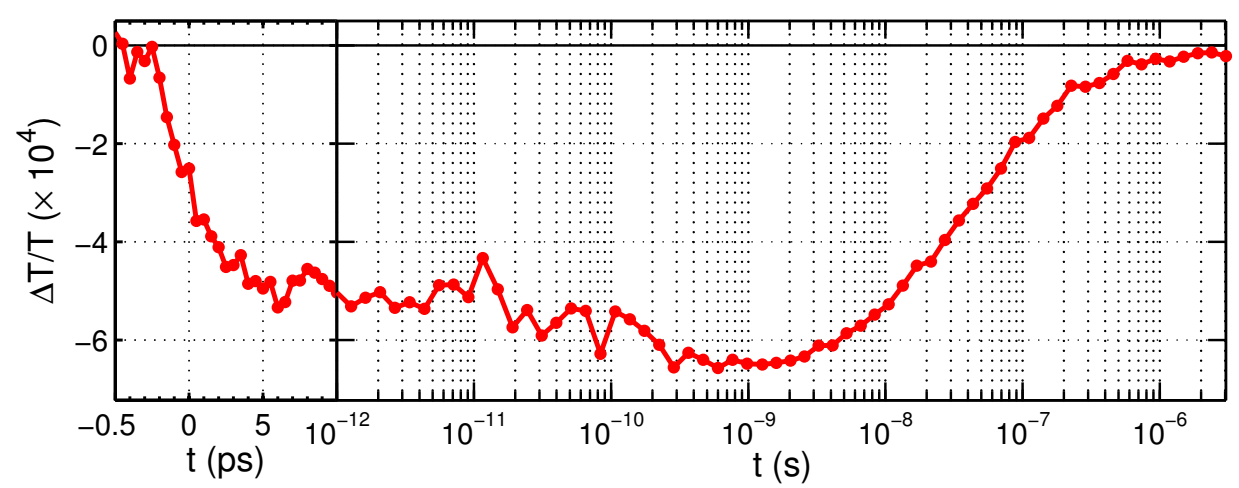

Figure 4.12: Combined fs and ps TA of PCDTBT:PCBM (1:4), probed at $800 \mathrm{~nm}$. 


\subsubsection{PCPDTBT}

Poly[2,6-(4,4-bis-(2-ethylhexyl)-4H-cyclopenta [2,1b;3,4-b]dithiophene)-alt-4,7(2,1,3-benzothiadiazole)] or more conveniently, PCPDTBT, is an extremely low-bandgap $\left(E_{g}=1.46 \mathrm{eV}\right.$ [60]) donoracceptor copolymer, with significant absorption to $830 \mathrm{~nm}$ - almost the optimum value for a traditional single junction solar cell. [64] The broad

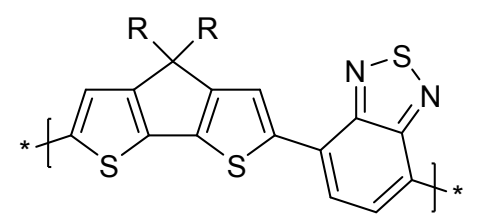

$R=$ 2-ethylhexyl absorption of PCPDTBT (especially when combined with the asymmetric fullerene $\mathrm{PC}_{71} \mathrm{BM}$,

Figure 4.13: PCPDTBT structure. where broken symmetry allows better visible absorption than $\mathrm{PC}_{61} \mathrm{BM}$ ) enables power conversion efficiencies of $5.5 \%$ when cast with a small fraction of alkanethiol to control solvent evaporation during spin-coating. [60] The blend used here uses the optimized 1:2 ratio and ODT cosolvent to maximize charge photoseparation.

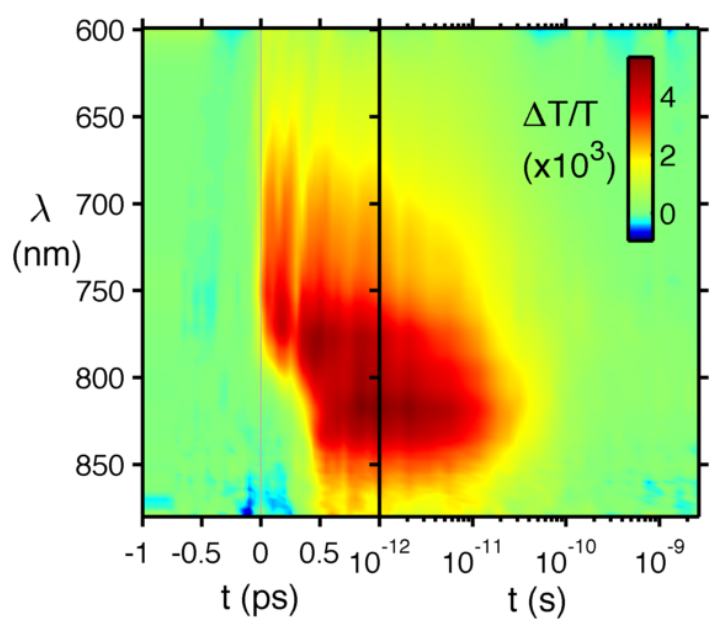

(a)

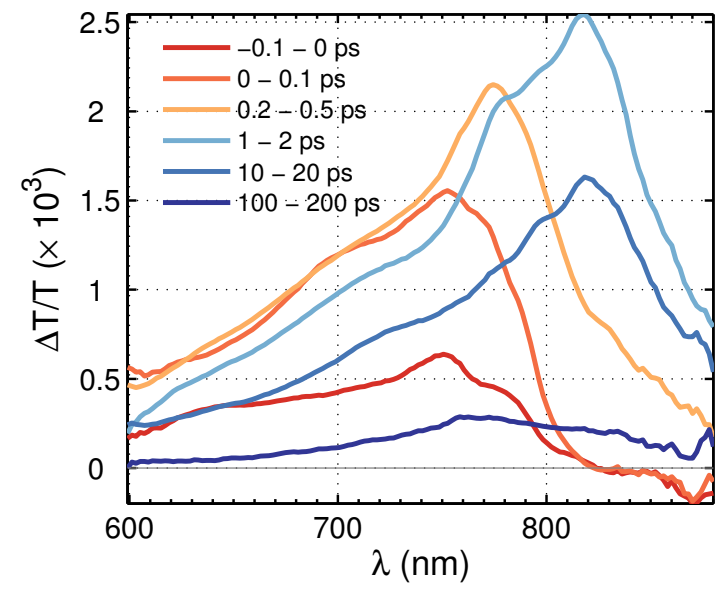

(b)

Figure 4.14: TA map (a) and spectra (b) of neat PCPDTBT film.

The TA spectrum of neat PCPDTBT is shown in Figure 4.14. The interesting looking structure below $800 \mathrm{~nm}$ at early times is in fact an artifact of our probe light source (NOPA), which was inefficiently tuned during the measurement, resulting in the presence of significant residual $800 \mathrm{~nm}$ light with different temporal characteristics to the amplified white light. The effect is particularly pronounced due to the absorption of $800 \mathrm{~nm}$ light from the ground state in PCPDTBT. However, 
we can still identify the excited state feature in our probed wavelength region as mostly GSB with some SE towards $850 \mathrm{~nm}$ (again based on quenching, solid state spectroscopy and by comparison with previously published work. [71,151])

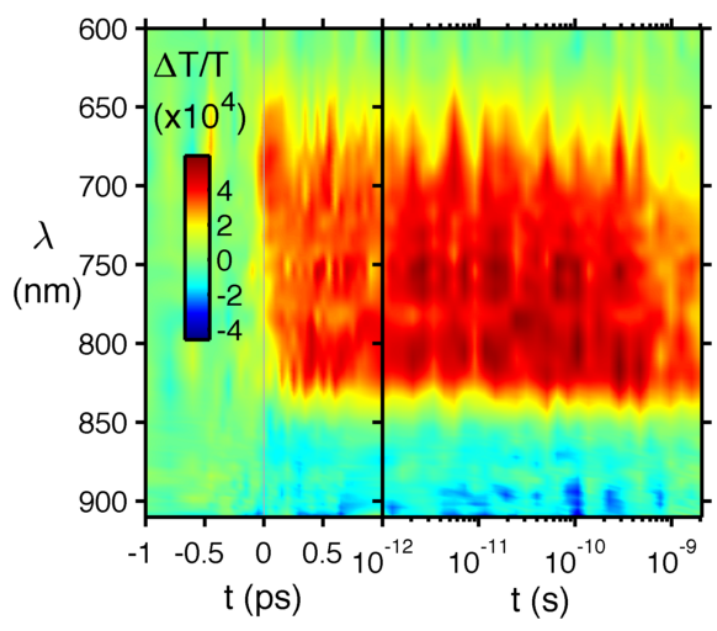

(a)

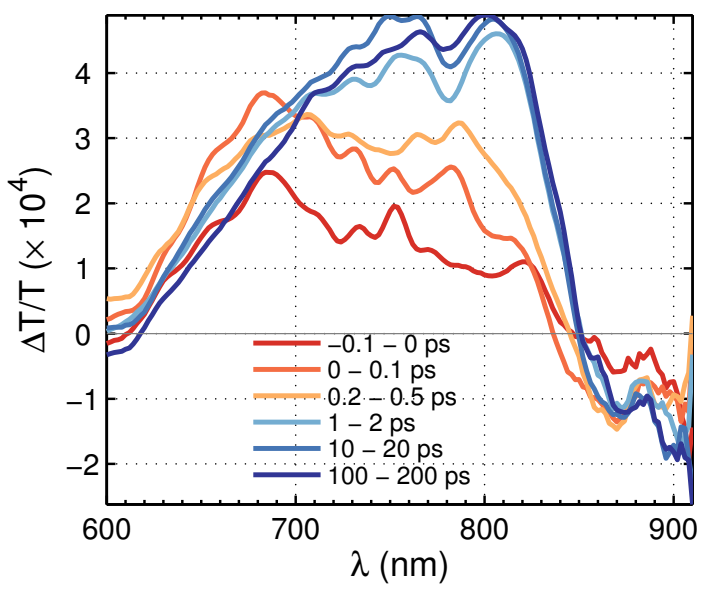

(b)

Figure 4.15: TA map (a) and spectra (b) of PCPDTBT:PCBM (1:2) film.

Upon blending with $\mathrm{PC}_{61} \mathrm{BM}$ (Figure 4.15) we again see rapid quenching of SE and the emergence of a long-lived PIA, in this case at the very edge of the range of our probe light source.

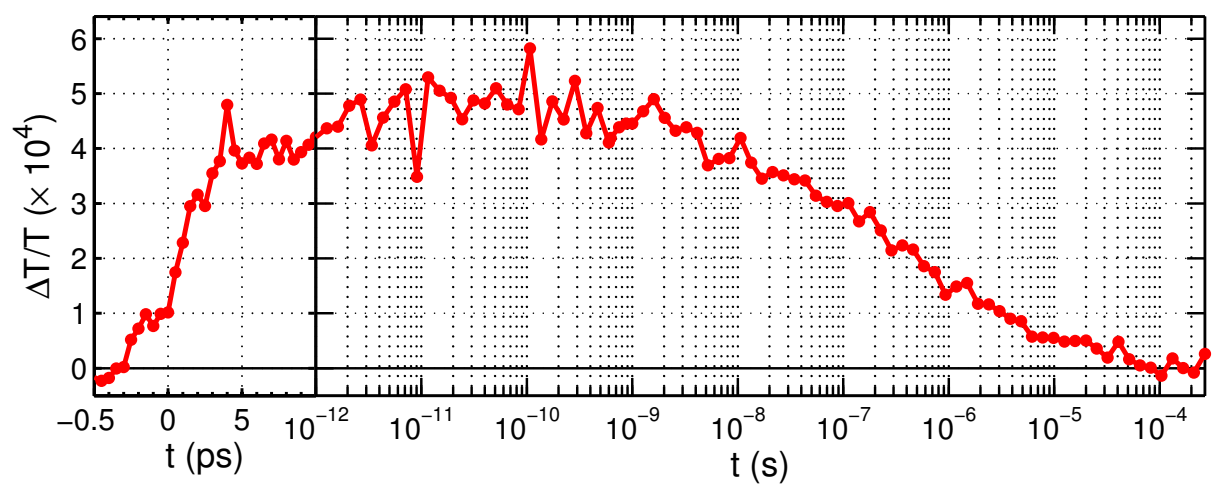

Figure 4.16: Combined fs and ps TA of PCPDTBT:PCBM (1:2), probed at $800 \mathrm{~nm}$.

PCPDTBT:PCBM is unique amongst our samples as the kinetics at $800 \mathrm{~nm}$ are probing the GSB, rather than a PIA (Figure 4.16). Nevertheless, while inverted, we see similar behaviour to the previous blends. The majority of the signal grows in several hundred femtoseconds, followed by some continued growth due to 
exction diffusion. On nanosecond timescales and beyond, all singlet excitons have decayed, and ideally the GSB amplitude is equal to photoinduced charge density. There has been some debate about the presence of long-lived triplet excitons in PCPDTBT:PCBM formed by spin mixing of interfacial CT states, that may contribute to the long lived GSB in our signal. When planning and conducting the measurement, the majority of published work indicated that triplet formation was reduced to negligible rates in optimized morphologies, such as obtained through the use of alkane dithiol cosolvents. [101,152] However, recent work [40] presents conflicting conclusions, which warrant caution when attributing signals to excited states in this system.

\subsubsection{MEH-PPV}

Poly[2-methoxy-5-(2-ethylhexyloxy)-1,4-phenylenevinylene], or MEH-PPV, is one of the earliest OPV polymers. As such, it has been (along with variants such as MDMO-PPV) the subject of extensive research, possibly second only to P3HT in terms of sheer number of OPV publications. With reported power conversion efficiencies on the

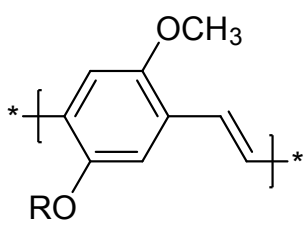

$R=$ 2-ethylhexyl order of $0.5 \%$, [147] MEH-PPV:PCBM is a relatively inefficient system, with a large bandgap Figure 4.17: MEH-PPV structure. (only absorbing above $\sim 560 \mathrm{~nm}[132]$ ) and low IQE of $40-50 \%$. [94,95]

Unfortunately, TA of the neat MEH-PPV film was omitted during data collection, though of course many published results are available. [126,153-155] In short, while MEH-PPV is fluorescent and exhibits SE in solution, almost none is seen in neat films due to interchain interactions. [153] The neat TA spectrum is therefore shortlived ( $<100 \mathrm{ps}$ and comprised of GSB below $\sim 550 \mathrm{~nm}$, and a broad PIA from $\sim 650-950 \mathrm{~nm}$.

MEH-PPV:PCBM was blended at a ratio of 1:4, with the resulting TA shown in Figure 4.18. In our wavelength range the signal is almost entirely PIA, with a peak at around $850 \mathrm{~nm}$.

The kinetics show $\approx 75 \%$ growth of the PIA within several hundred femtoseconds, followed by a gradual increase over the next $100 \mathrm{ps}$. On nanosecond scales 


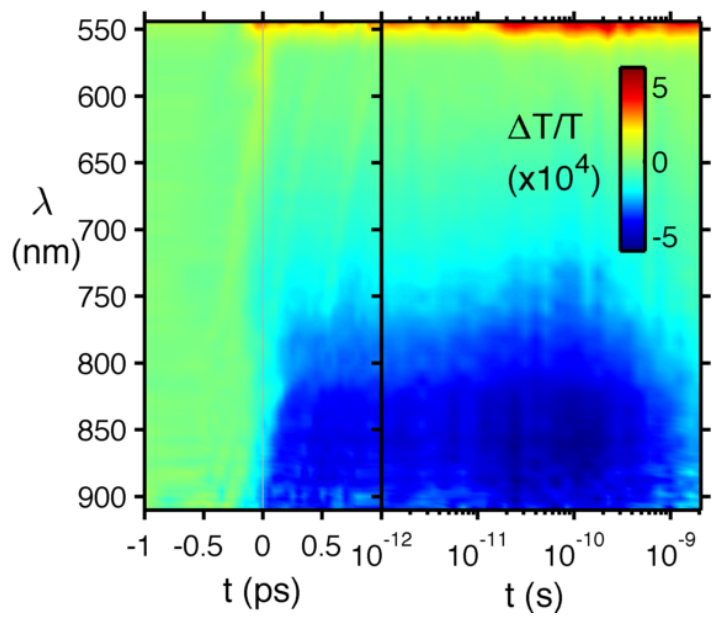

(a)

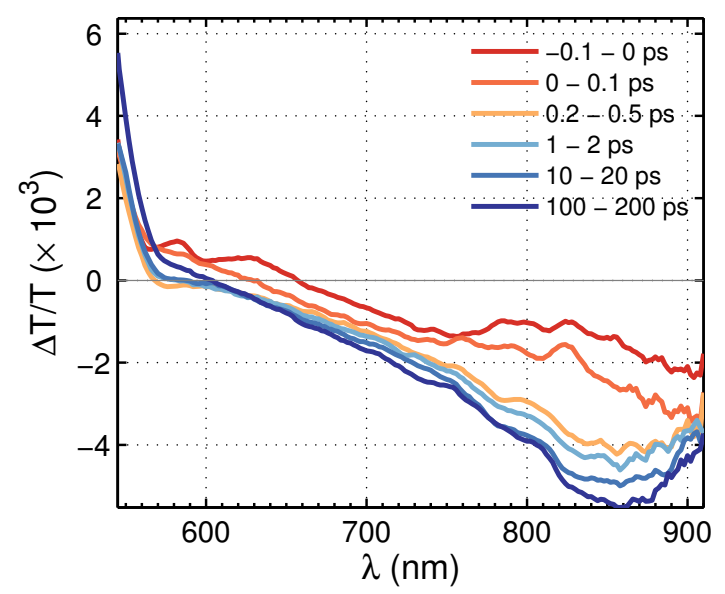

(b)

Figure 4.18: TA map (a) and spectra (b) of MEH-PPV:PCBM (1:4) film.

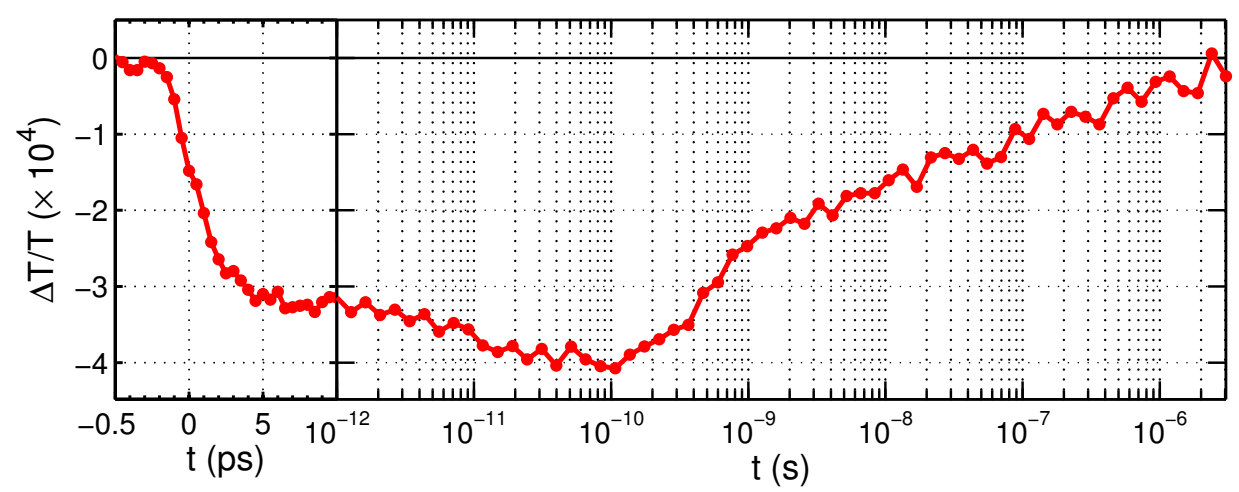

Figure 4.19: Combined fs and ps TA of MEH-PPV:PCBM (1:4), probed at $800 \mathrm{~nm}$. 
and beyond, the signal as probed at $800 \mathrm{~nm}$ is entirely due to photogenerated charges.

\subsection{Correlation of recombination rate with photocur- rent efficiency}

What can we directly determine about the efficiency of charge generation in our range of materials from the recombination kinetics presented in Section 4.3? A useful comparison may be with internal quantum efficiency (IQE). IQE is an excitation wavelength-dependent metric determined by normalizing the charge yield per incident photon by the absorption of incident photons in the active layer for each wavelength. Measurement of IQE is a subtle technique as allowances must be made for effects such as internal reflections and interference within the various device layers. [95]

\begin{tabular}{|l|c|c|}
\hline & $\begin{array}{c}\tau(800 \mathrm{~nm}) \\
/ 10^{-9} \mathrm{~s}\end{array}$ & $\begin{array}{c}\mathrm{IQE} \\
\text { (excited at 532 nm) }\end{array}$ \\
\hline P3HT:PCBM (as-cast) & 20 & $30 \%[156]$ \\
P3HT:PCBM (annealed) & 500 & $70 \%$ [156], 80\% [94] \\
PCDTBT:PCBM (1:4) & 45 & $90 \%$ [95], 90\% [148] \\
PCPDTBT:PCBM (1:2) & 200 & $65 \%$ [40] \\
MEH-PPV:PCBM (1:4) & 3 & $45 \%$ [94], 40-50\% [95] \\
\hline
\end{tabular}

Table 4.1: Comparing polaron lifetime with published IQE.

Table 4.1 shows the lifetime of photogenerated charges from our kinetics probed at $800 \mathrm{~nm}$, approximated by taking the time for the signal to decay to half of its maximum value. Also shown are representative values of IQE for those blends, taken from the literature. The two metrics are compared in Figure 4.20, which does not indicate strong correlation for our (admittedly small) set of materials.

Given the range of factors determining charge lifetime, particularly the dependence on charge density, it is not surprising that a single measurement of the recombination is unable to provide us with much information about the performance of a given material. We now move on to the density dependence of recombination, which is able to provide significantly greater insight. 


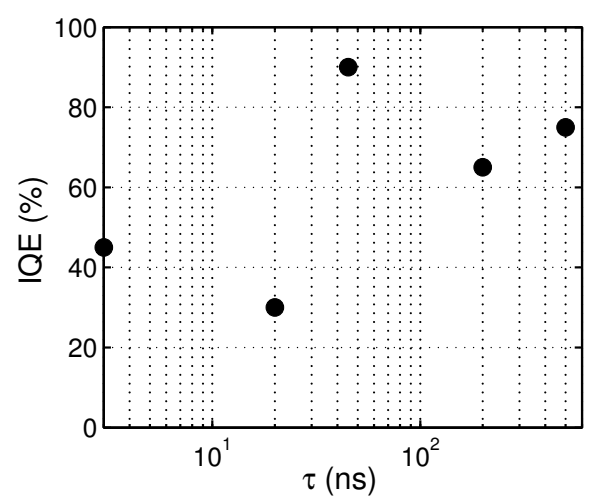

Figure 4.20: Comparison of reported IQE vs charge lifetime from TA kinetics for a range of polymer:PCBM blends.

\subsection{Fitted intensity-dependent TA}

As descibed in the introduction to this chapter, the different dependence of reombination of bound and free charge pairs on charge density has been used by Howard et al. to determine the efficiency of branching taking place shortly after photoexcitation. [101,102,157]

Since the CT thermalization time ( $\leq 500 \mathrm{fs}[96,158,159])$ is much faster than geminate recombination ( $>100$ ps, even at high intensities [115]) then any experiment measuring on timescales of $0.1 \mathrm{~ns}$ or greater can take the populations of bound and free charges as an intitial condition. It turns out the rate equations for a system where the bound and free charge pairs recombine monomolecularly and bimolecularly respectively can be solved, where the solutions for the time dependent populations in each state are: [102]

$$
\begin{aligned}
& \mathrm{CT}(t)=N_{0}\left(1-\Phi_{\text {free }}\right) \exp \left(-k_{\mathrm{CT} \rightarrow \mathrm{GS}} t\right) \\
& \operatorname{SSC}(t)=\left(\lambda \gamma t+\left(\Phi_{\text {free }} N_{0}\right)^{-\lambda}\right)^{-1 / \lambda} \\
& \mathrm{GS}(t)=N_{0}\left(1-\Phi_{\text {free }}\right)\left(1-\exp \left(-k_{\mathrm{CT} \rightarrow \mathrm{GS}} t\right)\right)+ \\
& N_{0} \Phi_{\text {free }}-\left(\left(\lambda \gamma t+\left(\Phi_{\text {free }} N_{0}\right)^{-\lambda}\right)^{-1 / \lambda},\right.
\end{aligned}
$$

where $\mathrm{CT}$ is the (bound) charge-transfer state population, $\mathrm{SSC}$ is the spatially separated ('free charge') population (counting electrons only), GS is the population that has relaxed back to the ground state, $\gamma$ the density-dependent recombination rate constant, $\lambda+1$ the order of the bimolecular recombination, $k_{\mathrm{CT} \rightarrow \mathrm{GS}}$ the rate of geminate recombination, $\Phi_{\text {free }}$ the fraction of free charges from the initial branching event, and finally, $N_{0}$ is the total initial charge population. 
Charge recombination kinetics were collected at room temperature using an $800 \mathrm{~nm}$ probe, as described Chapter 2 and in the previous section. Initial charge density was varied by adjusting the energy density of the pump pulse with a variable attenuator. Sample film thickness was measured using a Dektak profilometer and optical density determined by UV-vis spectroscopy. These values were used along with the fluence (photons per square centimetre per pulse) of the excitation pulse to calculate the initial density of photogenerated charges for each dataset (shown in figure insets).

The resulting intensity dependent kinetics along with fits using the model described above are shown in Figure 4.18. Fitted parameters are shown, where $f$ is the fraction of nongeminate recombination (presented as $\Phi_{\text {free }}$ in the main text).

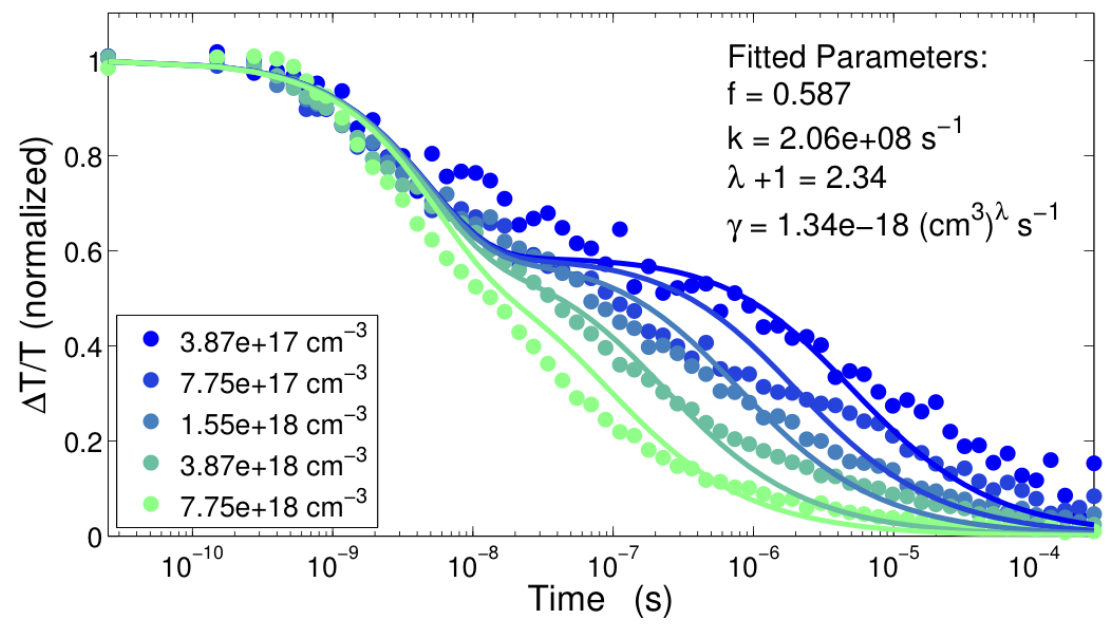

(a) P3HT:PCBM (1:1), as-cast

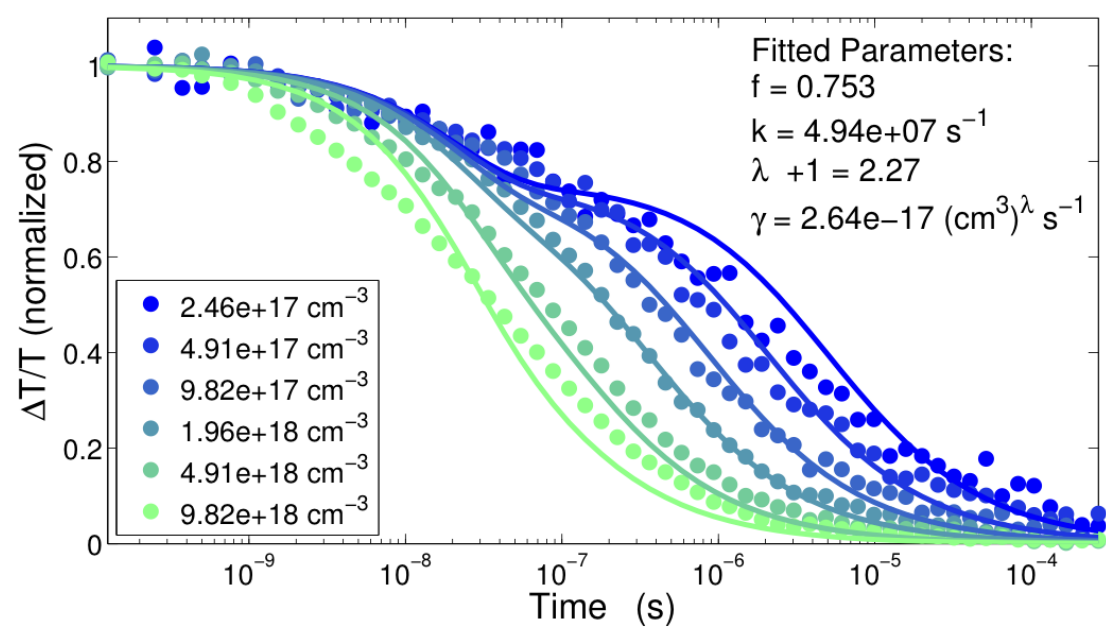

(b) P3HT:PCBM (1:1), annealed 


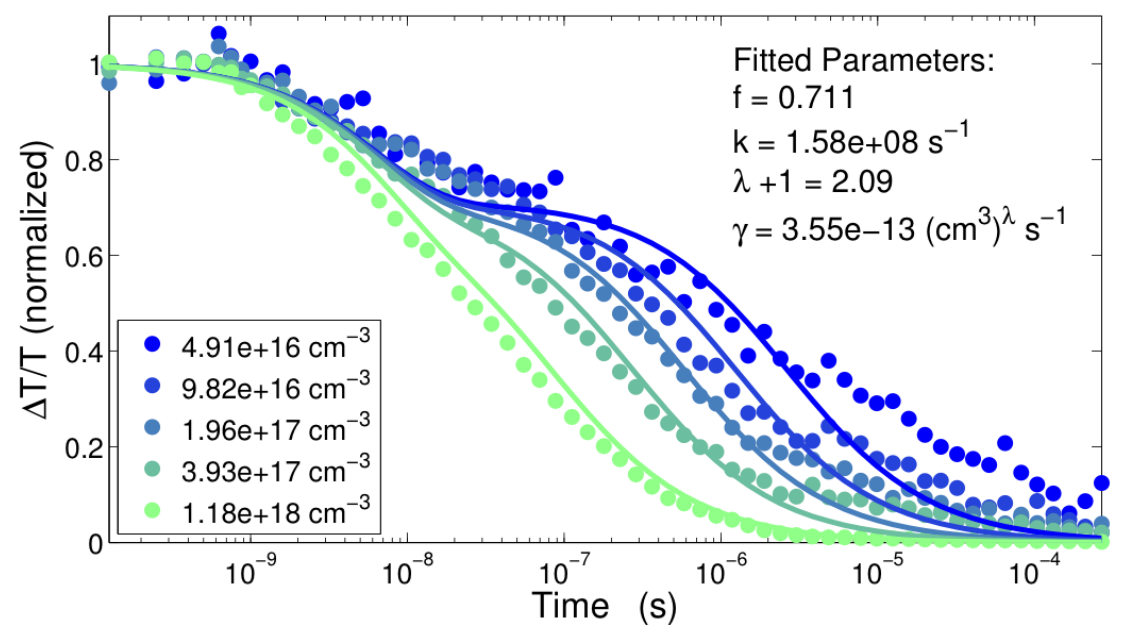

(c) P3HT:PCBM (1:1), $355 \mathrm{~nm}$ excitation

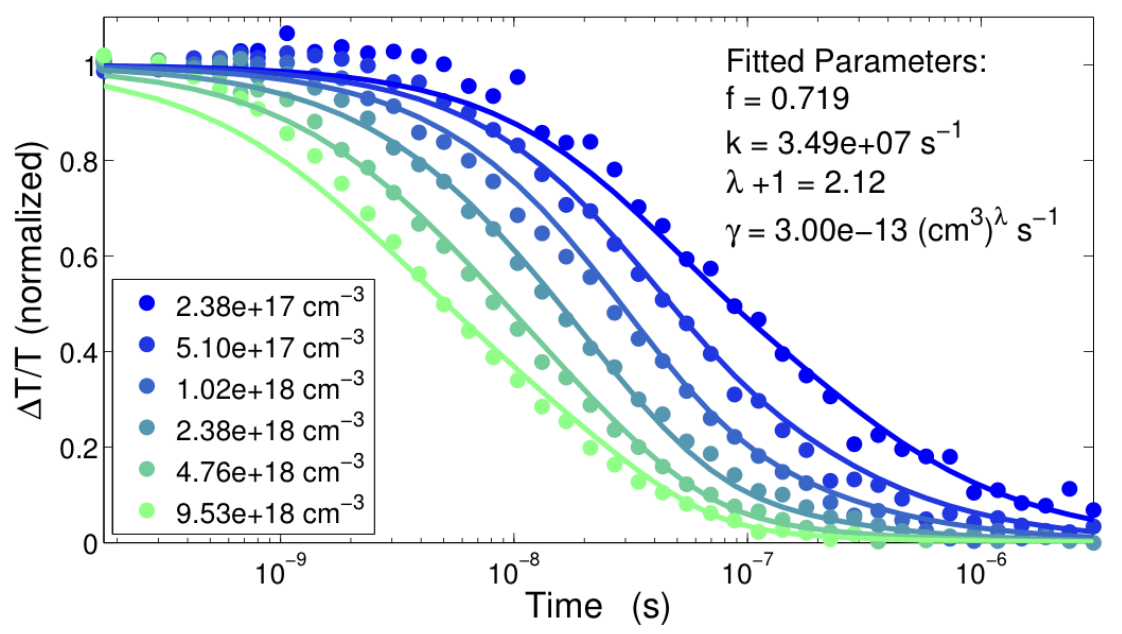

(d) PCDTBT:PCBM (1:4)

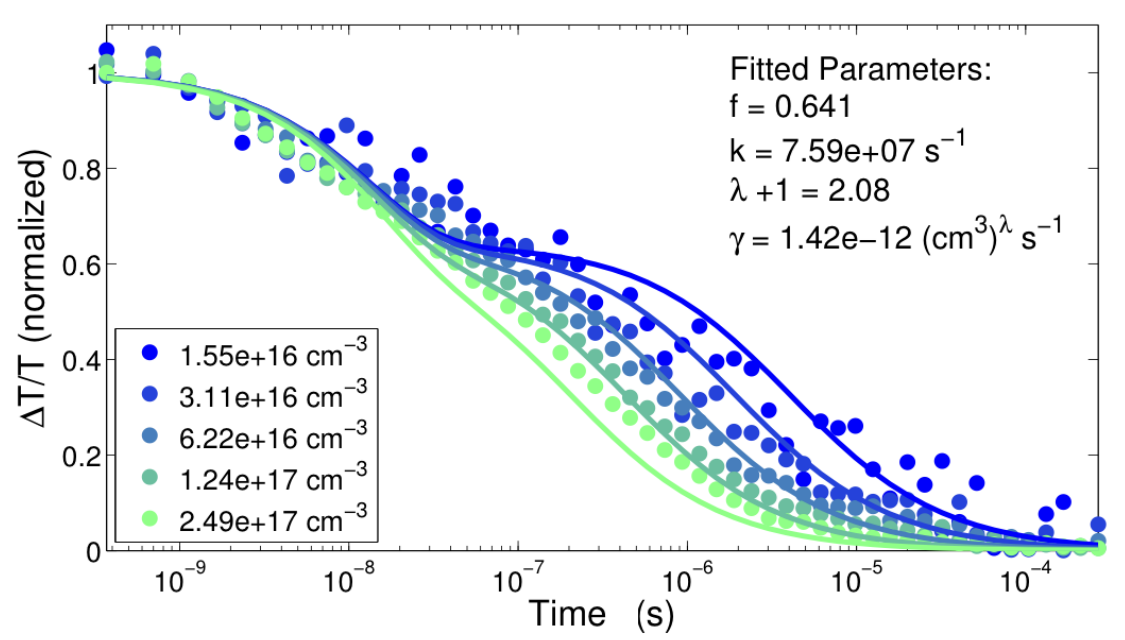

(e) PCPDTBT:PCBM (1:2) 


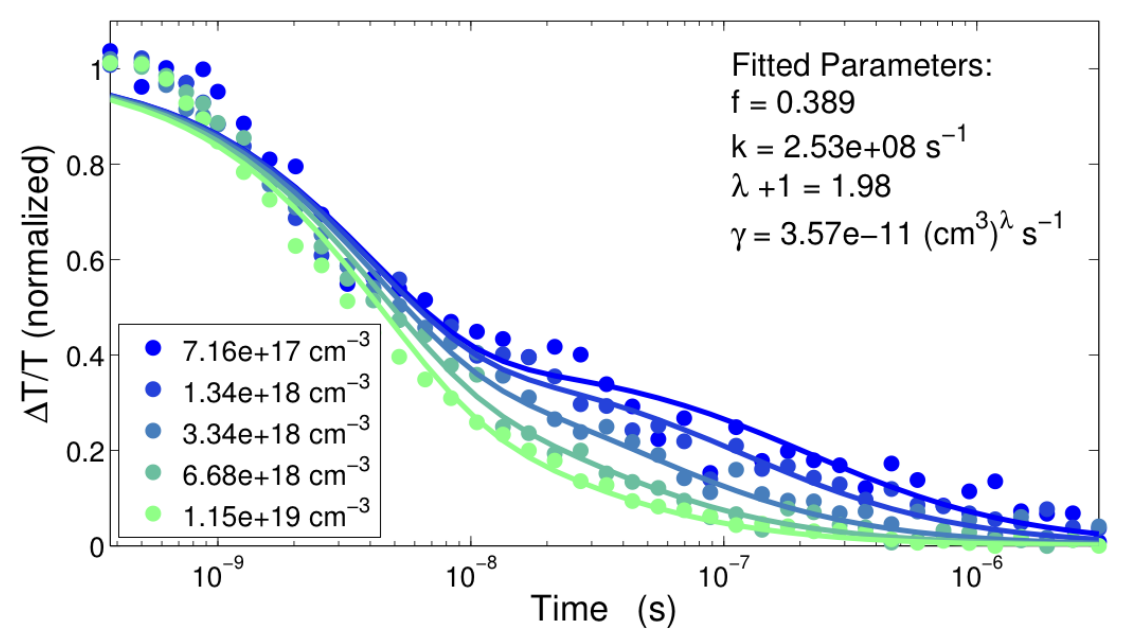

(f) MEH-PPV:PCBM (1:1)

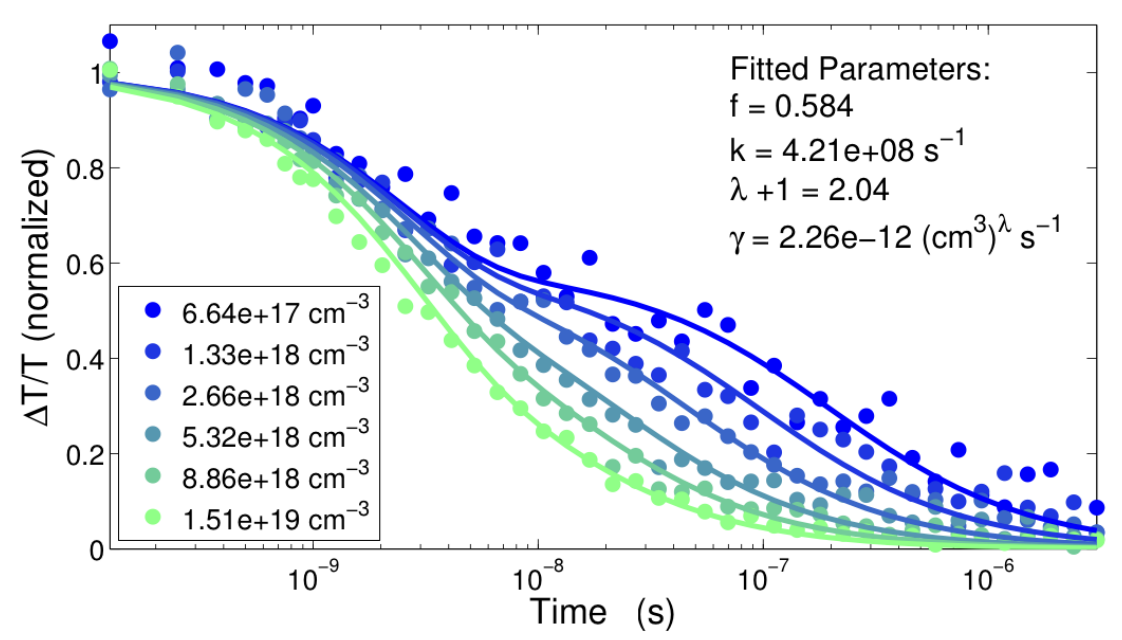

(g) MEH-PPV:PCBM (1:4)

Figure 4.18: Intensity dependent TA kinetics (circles) and fits (solid lines) for various materials, excited at $532 \mathrm{~nm}$ and probed at $800 \mathrm{~nm}$. Values shown in solid boxes indicate initial charge densities, calculated from pump energy density, film thickness, and optical density. 
As can be seen in Figure 4.19, MEH-PPV:PCBM blends exhibit appreciable recombination within the time resolution of this experiment. The fraction of subnanosecond recombination was quantified as $(1-0.504)$ and $(1-0.572)$ for the 1:1 and 1:4 blends respectively, as discussed further in Section 5.5 of the next chapter.

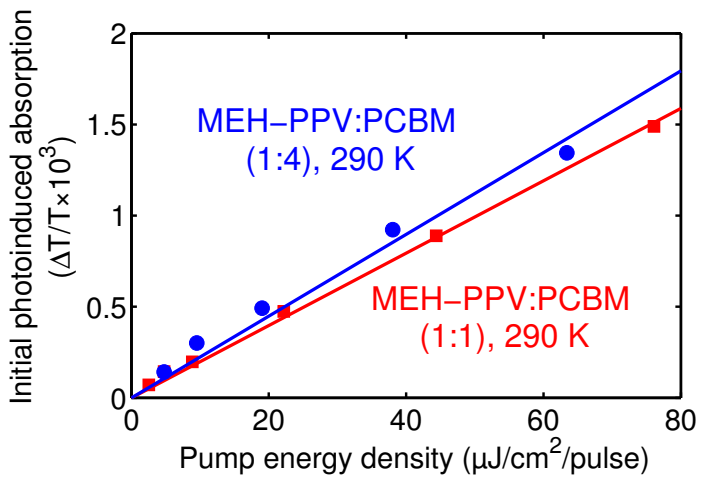

Figure 4.19: Initial PIA in MEH-PPV:PCBM is close to linear with pump energy density

For both 1:1 and 1:4 blends, the magnitude of the initial photoinduced absorption signal is close to linear with respect to the initial pump intensity (see Figure 4.19). This indicates that negligible bimolecular recombination takes place within the convolution period, allowing us to attribute the missing signal entirely to bound charge pairs, and apply a correction to $\Phi_{\text {free, }}$ the yield of free charge pairs. These corrected values are shown with the rest of the fitted parameters in Table 4.2 .

If we now take our newly determined values of free charge yield $\Phi_{\text {free }}$ and revisit the comparison with IQE, we see an improved correlation with overall device performance. However it is still a somewhat tenuous connection, as IQE does not uniquely probe the initial charge generation process. In Chapter 5 , we describe the development of a new spectroscopic method that is able to probe the photogenerated charge pair immediately following the charge-transfer step.

Adjusted to account for sub-picosecond recombination 


\begin{tabular}{|l|c|c|c|c|c|}
\hline & $\Phi_{\text {free }}$ & $\begin{array}{c}k_{\mathrm{CT} \rightarrow \mathrm{GS}} \\
/ 10^{7} \mathrm{~s}^{-1}\end{array}$ & $\lambda+1$ & $\begin{array}{c}\gamma \\
/\left(\mathrm{cm}^{3}\right)^{\lambda} \mathrm{s}^{-1}\end{array}$ & $\begin{array}{c}\text { IQE } \\
(\text { excited at 532 nm) }\end{array}$ \\
\hline P3HT:PCBM (as-cast) & 0.587 & 20.6 & 2.34 & $1.34 \times 10^{-18}$ & $30 \%$ [156] \\
P3HT:PCBM (annealed) & 0.753 & 4.94 & 2.27 & $2.64 \times 10^{-17}$ & $70 \%$ [156], 80\% [94] \\
P3HT:PCBM (355 nm ex.) & 0.711 & 15.8 & 2.09 & $3.55 \times 10^{-13}$ & \\
PCDTBT:PCBM (1:4) & 0.719 & 3.49 & 2.12 & $3.00 \times 10^{-13}$ & $90 \%$ [95], 90\% [148] \\
PCPDTBT:PCBM (1:2) & 0.641 & 7.59 & 2.08 & $1.42 \times 10^{-12}$ & $65 \%$ [40] \\
MEH-PPV:PCBM (1:1) & $0.260^{\mathrm{i}}$ & 25.3 & 1.98 & $3.57 \times 10^{-11}$ & \\
MEH-PPV:PCBM (1:4) & $0.408^{\mathrm{i}}$ & 42.1 & 2.04 & $2.26 \times 10^{-12}$ & $45 \%$ [94], 40-50\% [95] \\
\hline
\end{tabular}

Table 4.2: Fitted parameters from kinetic model

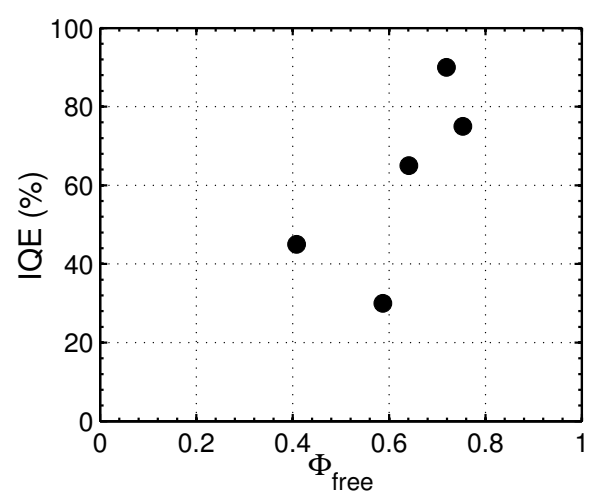

Figure 4.20: Comparison of reported IQE vs free charge yield from excitationdensity dependent recombination fits 


\subsection{Reconciliation of optical and electronic probes of recombination}

Various measurements of charge density and recombination often seem to report conflicting rates of charge lifetime in OPVs, with discrepancy often between the 'fast' measurements (such as femtosecond spectroscopy) and slower measurements, such as transient photovoltage or time of flight techniques.

$\mathrm{We}^{\mathrm{ii}}$ decided to investigate whether there was an effect due to charge polarization or other phenomena that may only be taking place under extended illumination or in operating devices. We therefore undertook TA on a functioning OPV device (P3HT:PCBM) using a $20 \mu$ s long pulse from a $480 \mathrm{~nm}$ LED as an excitation source, enabling simultaneous measurement of $V_{\mathrm{OC}}$ and $\Delta T / T$.

The delay between the LED switching off and the probe arrival was controlled by an electronic delay generator. The transient absorption probe was an $800 \mathrm{~nm}$ pulse in reflection mode. In order to allow for charges to sufficiently recombine between pulses, we operated our laser at a reduced repetition rate of $187.5 \mathrm{~Hz}$, which was the time required for $V_{\mathrm{OC}}$ to decay to less than half of its initial value.

The results are shown in Figure 4.21, where TA using the LED excitation is shown with the device both in open-circuit and short-circuit configuration. Also shown are the $V_{\mathrm{OC}}$ decay that was collected simultaneously with TA, and traditional laser-excitation TA using our picosecond laser at $532 \mathrm{~nm}$ as a pump.

We see that the TA kinetics measured under LED excitation are similar to those collected with picosecond excitation, but seem to be significantly faster than decay of the open-circuit voltage. No change is observed upon changing the device from open-circuit to short-circuit configuration.

The situation becomes clearer however, when we rescale our TA data to charge density, using either the fluence of incident pump photons and measured film thickness or reported figures of polaron absorption cross section ${ }^{\text {iii. [ [102] }}$

We must also convert $V_{\mathrm{OC}}$ to charge density, which is expected to behave as $n=n_{\mathrm{i}} \exp \left(\frac{\mathrm{eV}_{\text {mathrmOC }}}{2 \mathrm{kT}}\right)$. [160] Upon combining the rescaled datasets, we see that

\footnotetext{
${ }^{i i}$ This work was conducted in collaboration with Lionel Hirsch and Mamatimin Abbas at the Laboratoire de l'Integration du Materiau au Systeme in Bordeaux, France.

iii Alternately we could even measure polaron absorption cross section ourself using the new method introduced in Chapter 6!
} 


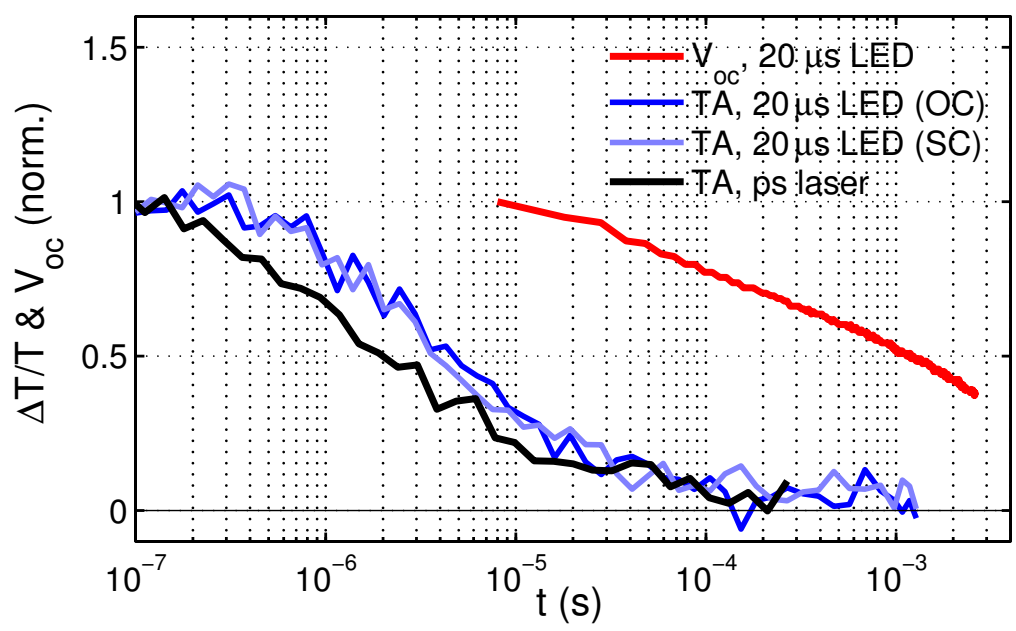

Figure 4.21: $V_{\mathrm{OC}}$ decay and transient absorption collected simultaneously under pulsed LED excitation of a functioning P3HT:PCBM device. Also shown is TA of a P3HT:PCBM film collected with picosecond laser excitation.

the discrepancy in recombination timescales is effectively resolved, as shown in Figure 4.22

By combining the two measurements, we are able to fully monitor the progession of excitations in OPVs from creation to recombination, over an unprecedented range of resolved time $\left(10^{-13}-10^{-2} \mathrm{~s}\right)$ and charge density $\left(10^{13}-10^{19} \mathrm{~cm}^{-3}\right.$ and above). 


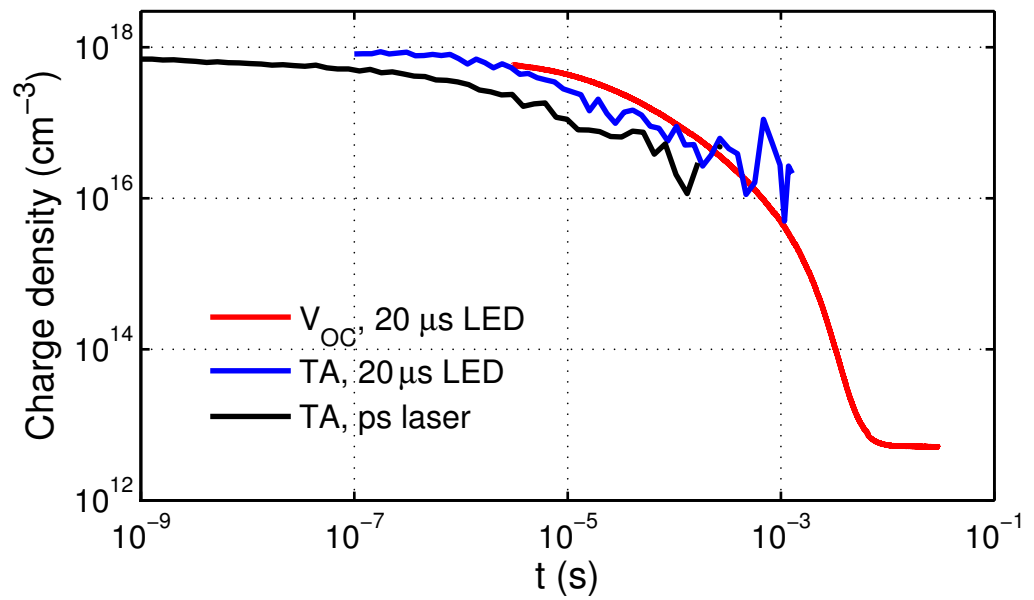

Figure 4.22: Upon converting TA and $V_{\mathrm{OC}}$ decay to population density, we see much better agreement in charge decay.

\subsection{Conclusions}

We have presented broadband TA of a range of OPV blends of varying performance, generating a library of samples amongst which to compare results from several spectroscopic techniques. The dependence of recombination rate on intensity was used to measure the yield of well separated charges, which provides a better indication of device efficiency than simple reocombination measurements such as charge lifetime.

Finally, we briefly discussed the connection between electronic and optical measurements of charge density, and show how they can be reconciled to allow measurement of charge recombination over an exceptional range of timescales $\left(10^{-13}-10^{-2} \mathrm{~s}\right)$ and charge densities $\left(10^{13}-10^{19} \mathrm{~cm}^{-3}\right.$ and above). 


\title{
Chapter 5
}

\section{Distance distributions of photogenerated charge pairs in organic photovoltaic cells}

\begin{abstract}
iStrong Coulomb interactions in organic photovoltaic (OPV) cells dictate that charges must separate over relatively long distances in order to circumvent geminate recombination and produce photocurrent. In this chapter, we measure the distance distributions of thermalized charge pairs by accessing a regime at low temperature where charge pairs are frozen out following the primary charge separation step and recombine monomolecularly via tunneling. The exponential attenuation of tunneling rate with distance provides a sensitive probe of the distance distribution of primary charge pairs, reminiscent of electron transfer studies in proteins. By fitting recombination dynamics to distributions of recombination rates, we identified populations of charge-transfer states and well-separated charge pairs. For the wide range of materials we studied, the yield of separated charges in the tunneling regime is strongly correlated with the yield of free charges measured via their intensity dependent bimolecular recombination dynamics at room temperature. We therefore conclude that populations of free charges are established via long-range charge separation within the thermalization timescale,
\end{abstract}

${ }^{\mathrm{i}}$ This work has been accepted for publication in the Journal of the American Chemical Society (pending minor revisions) as Distance distributions of photogenerated charge pairs in organic photovoltaic cells - Alex J. Barker, Kai Chen and Justin M. Hodgkiss. 
thus invoking early branching between free and bound charges across an energetic barrier. Subject to assumed values of the electron tunneling attenuation constant, we find critical charge separation distances of $\sim 3-4 \mathrm{~nm}$ in all materials. In some blends, large fullerene crystals can enhance charge separation yields, however, the important role of the polymers is also highlighted in blends that achieved significant charge separation with minimal fullerene concentration. We expect that our approach of isolating the intrinsic properties of primary charge pairs will be of considerable value in guiding new material development and testing the validity of proposed mechanisms for long-range charge separation.

\subsection{Introduction}

Rational design of effective OPV materials requires understanding how interfacial charge pairs achieve sufficient separation to overcome their mutual Coulombic attraction. Simple application of Coulomb's law suggests that in a medium with a dielectric constant of 3.5 , point charges must achieve a separation of $\sim 4 \mathrm{~nm}$ before their interaction energy is less than the intrinsic energetic disorder of typical organic materials $(0.1 \mathrm{eV})$. [37,67,161] Entropic considerations and charge delocalization will favor charge separation, nevertheless, charge pairs that fail to separate by more than a few nanometers are likely to collapse into tightly bound charge transfer (CT) states and recombine monomolecularly, often radiatively. $[80,102,114,115,155,162-164]$ The observed sensitivity of OPV device efficiency to blend morphology $[60,165]$ can be attributed to the need for donor and acceptor phases to be large enough to support formation of separated charges (SCs); blends that are too finely intermixed result in terminal CT states forming at the expense of extractable free charges. [77,102,162,166]

In spite of the emergence of models describing different relationships between excitons, CT states and SCs on a potential energy surface spanning several nanometers from the donor/acceptor interface (see Section 1.3.3), critical parameters including distances have yet to be experimentally established.

Measured phase sizes from optimized blends provide some guidance on critical length scales for interfacial charge separation, however phase optimization is also coupled to exciton transport and charge extraction processes. Direct spectroscopic probes of distances are challenging because only tightly bound radiatively coupled CT states provide optically distinct signatures that identify their distance. More 
widely observed polaron absorption signatures are generally insensitive to the proximity of other charges.

Several recent attempts to resolve dynamics of charge pair distances have targeted phenomena that depend on the electric field created by charge separation. Time-resolved second harmonic generation spectroscopy has been used to resolve photovoltage generation on various timescales, however symmetry requirements restrict the technique to planar bilayer devices. $[99,167,168]$ Electroabsorption (Stark shift) effects are observed in dye-sensitized [169] and organic bulk heterojunction photovoltaic devices. [35] Their magnitude can be related to charge pair distances, leading to critical distances on the order of $4 \mathrm{~nm}$ for efficient OPVs, [35] however, this analysis requires that optically active components have a very sharp absorption edge so that the derivative-like electroabsorption features can be clearly identified and extracted from overlapping polaron, bleaching, and stimulated emission features. Finally, simultaneous measurements of dielectric and electron spin-resonance susceptibilities for OPVs revealed $\sim 4 \mathrm{~nm}$ charge localization lengths at cryogenic temperatures. [170]

Here, we present a new approach for determining the distance distribution of initially thermalized charge pairs. At low temperature, we are able to access a regime, previously identified in neat polymer films, [125] where all charge pairs are effectively frozen following the primary charge transfer step and recombine slowly via monomolecular tunneling. The exponential distance dependence of tunneling provides a sensitive probe of the distance distribution of initial charge pairs. For the wide range of OPV blends we probed, we find that dynamics are well fit to a bimodal distribution of tunneling recombination rates, consistent with branching between populations of CT states and SCs within the thermalization timescale. We observe a universal link between the free charge yield measured at room temperature and the yield of SCs frozen at low temperature. Our measurements confirm that free charge yields are established via the charge pairs that achieve an estimated $\sim 3-4 \mathrm{~nm}$ separation within the thermalization timescale. We also resolve the effect of polymer and fullerene components on the primary charge separation step. 


\subsection{Experimental details}

All materials were commercially sourced and spin-coated onto spectrosil fused silica substrates as described in Section 4.2. All samples were measured under dynamic vacuum and, where indicated, cooled to $10 \mathrm{~K}$ by a closed-cycle helium cryostat (CTI-Cryogenics).

Charge recombination was measured via transient absorption (TA) spectroscopy. 532 (or 355) nm excitation pulses were generated from the $2^{\text {nd }}\left(3^{\text {rd }}\right)$ harmonic of a Q-switched Nd:YVO 4 laser (AOT-YVO-25QSP, $700 \mathrm{ps)} \mathrm{and} \mathrm{filtered} \mathrm{to} \mathrm{the} \mathrm{fluences}$ indicated. A portion of the $800 \mathrm{~nm}$ output of an amplified Ti-sapphire (SpectraPhysics Spitfire, $100 \mathrm{fs}, 3 \mathrm{kHz}$ ) was used as a probe after splitting to introduce a reference channel for improved signal sensitivity. The transmitted probe light was spectrally dispersed using a fibre-coupled spectrograph and read out at $3 \mathrm{kHz}$ using a dual channel linear photodiode array. The pump-probe delay was electronically varied by a delay generator. Additional measurements of fast recombination in MEH-PPV:PCBM blends used the $100 \mathrm{fs}, 532 \mathrm{~nm}$ output of a parametric amplifier (TOPAS) as an excitation source, with the pump-probe delay varied by use of a retroreflector mounted on a motorized delay stage. Approximately 10,000 shot pairs were averaged for each time point and repeated over $\sim 20$ scans. Some variation was needed to account for signal strength at different intensities.

Data fitting was carried out in MATLAB (MathWorks), using the Nelder-Mead algorithm to minimize the sum of squares of the deviation between the measured data and the tunneling and intensity dependent decay models described. For both models, four parameters were fitted, and equal weighting was given to each order of magnitude of pump-probe delay.

\subsection{Results and discussion}

Our approach to measuring photogenerated charge pair distances requires accessing a regime where even SCs are frozen and slowly recombine monomolecularly via tunneling. It is well known that the preceding charge photogeneration step is not impeded at low temperature because excitons have sufficient excess thermal energy to reach interfaces before they relax. $[33,98,171]$ Figure 5.1 presents several independent verifications that we can access the monomolecular tunneling recombination regime. 


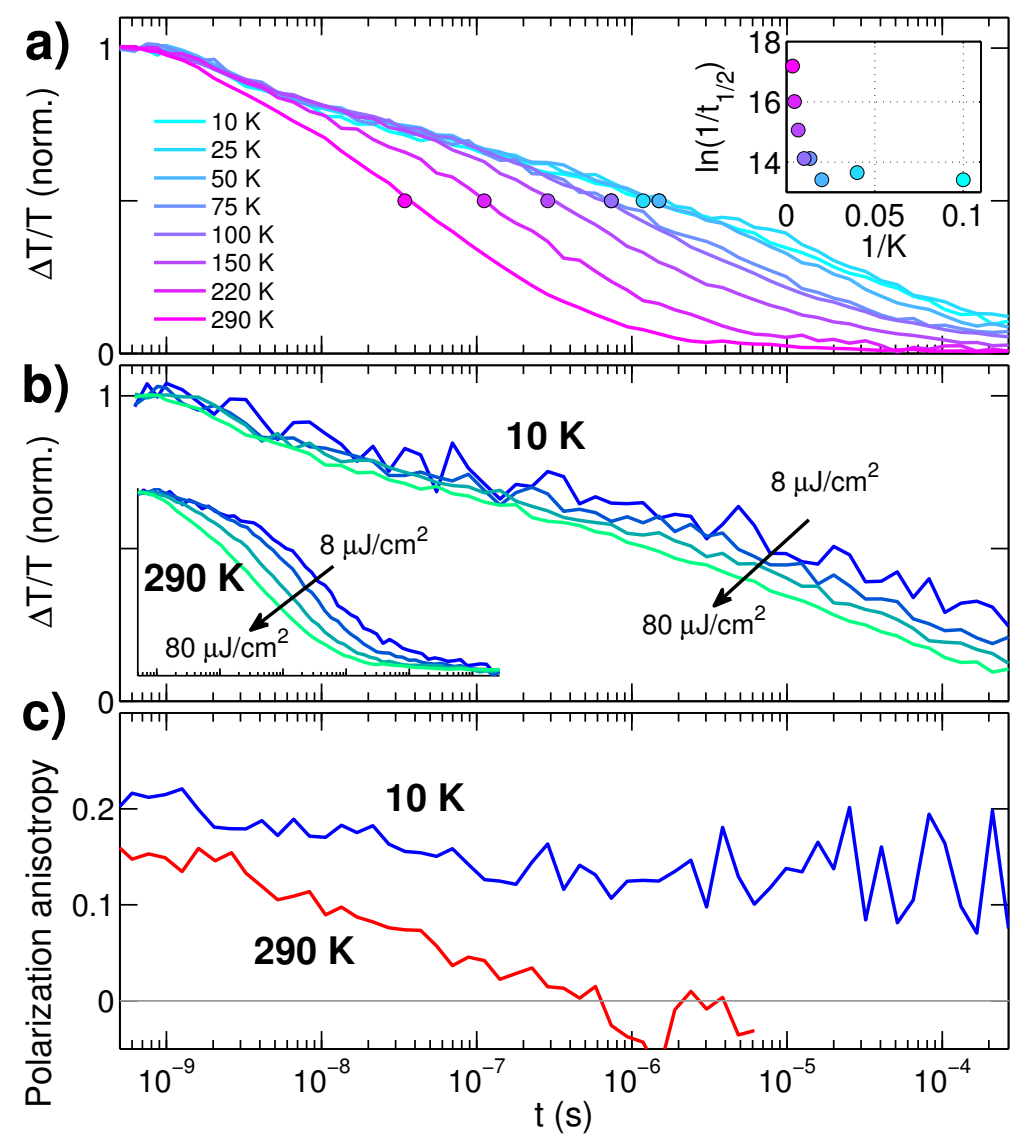

Figure 5.1: Recombination dynamics for a vapor annealed P3HT:PC ${ }_{61} \mathrm{BM}_{\text {blend }}$ film excited at $532 \mathrm{~nm}$ and probed at $800 \mathrm{~nm}$. a) Temperature dependent recombination ( $80 \mu \mathrm{J} \mathrm{cm}^{-2}$ excitation), with corresponding $t_{1 / 2}$ values represented in an Arrhenius type plot in the inset. b) Intensity dependent recombination for the same film at $10 \mathrm{~K}$ and $290 \mathrm{~K}$. c) Polarization anisotropy decay for the same film measured at $10 \mathrm{~K}$ and $290 \mathrm{~K}, 16 \mu \mathrm{J} \mathrm{cm}^{-2}$ excitation. 
Figure 5.1a shows the temperature dependent charge recombination dynamics for a vapor annealed P3HT:PCBM blend film, probed via the polymer hole polaron absorption signature in the near IR. $[77,102,162,172]$ The probed time range commences beyond that of exciton dynamics (see Section 4.3 for broadband TA measurements on faster timescales) and captures recombination of virtually the entire charge population. At $290 \mathrm{~K}$, bimolecular recombination dominates due to the high yield of free (mobile) charges photogenerated, as evidenced by the strongly intensity dependent $290 \mathrm{~K}$ recombination dynamics in Figure $5.1 \mathrm{~b}$ (inset). [102] This behavior typifies the active layer of an efficient OPV device except that in devices, free charges are extracted by the electrodes in competition with bimolecular charge recombination. [145] In the bimolecular recombination regime, recombination dynamics are non-geminate and do not directly reflect the properties of initially photogenerated charge pairs. As the temperature decreases, recombination slows and below $\sim 50 \mathrm{~K}$, we observe a temperature-independent regime of activationless charge recombination. The observed transition temperature is similar to previous observations of recombination in neat P3HT films owing to the intrinsic polymer-based activation barrier for polaron hopping in semiconducting polymers. [125] Figure 5.1b shows that the distinctive intensity dependence observed at $290 \mathrm{~K}$ (inset) is largely lost at $10 \mathrm{~K}$ since bimolecular charge recombination is thermally shut off. Instead, the predominantly monomolecular decay is attributed to charges being thermally trapped as geminate charge pairs near the site of their initial generation. Thus, charge pairs that would otherwise be free at room temperature remain frozen as SCs at low temperature. The residual weak intensity dependence at high intensity may result from charge delocalization at low temperature. [170] Finally, the weak polarization anisotropy decay shown in Figure 5.1c confirms that charges are effectively immobile at $10 \mathrm{~K}$, in contrast to the complete loss of polarization anisotropy associated with mobile charges at $290 \mathrm{~K}$.

To summarize this section; the temperature-, intensity-, and polarizationresolved TA measurements in Figure 5.1 verify that below $50 \mathrm{~K}$, charge pairs are effectively immobile beyond the ps thermalization timescale, leading to eventual monomolecular recombination, even for SCs.

The Arrhenius type plot in Figure 5.1a (inset) is reminiscent of DeVault and Chances renowned observation of temperature independent electron transfer (ET) in photosynthetic proteins at low temperature. [173,174] Their experiment 
stimulated theoretical efforts to put Marcus theory into a quantum mechanical framework to describe long-range electron tunneling mediated by quantum modes. $[175,176]$ Building on prior theories of polaron transfer in solids, [177] a semiclassical model emerged that expresses the rate of electron transfer between a weakly coupled donor and acceptor as; [175] [178]

$$
k_{E T}=\frac{2 \pi}{\hbar} \frac{\left|V_{D A}\right|^{2}}{\sqrt{4 \pi \lambda_{0} k_{B} T}} \sum_{\nu^{\prime}} e^{-S} \frac{S^{\nu^{\prime}}}{\nu^{\prime} !} \exp \left(\frac{-\left(\lambda_{0}+\nu^{\prime} \hbar \omega+\Delta G^{0}\right)^{2}}{4 \lambda_{0} k_{B} T}\right),
$$

where $V_{D A}$ is the electronic coupling between donor and acceptor states, $\lambda_{0}$ is the external reorganization energy, $\omega$ is the effective frequency of the coupled quantum mode, $S$ is the effective Huang-Rhys factor, $\nu^{\prime}$ is the vibrational quantum number on the product surface, $\Delta G^{0}$ is the driving force, and $k_{B} T$ is the thermal energy. Equation 1 predicts quantitatively correct electron transfer rates, including the virtual elimination of temperature dependence at thermal energies substantially below the energy of the effective quantum mode that promotes electron transfer. In this regime, the rate of electron transfer is most sensitive to the electronic coupling, which is exponentially dependent on distance owing to the exponential character of wavefunctions in the tunneling region;

$$
V_{D A}=V_{0} \exp \left(\frac{-\beta\left(R_{D A}-R_{0}\right)}{2}\right) .
$$

Here, the electronic coupling at a given donor-acceptor distance, $R$, is referenced to the coupling $\left(V_{0}\right)$ at a distance of $R_{0}$. The scaling factor, $\beta$, describes the rate of attenuation with distance. Assuming negligible distance dependence in other terms of equation 5.1, the distance dependence of $k_{E T}$ is;

$$
k_{D A}=k_{0} \exp \left(-\beta\left(R_{D A}-R_{0}\right)\right) .
$$

Extensive measurements in proteins featuring redox labels at well defined distances have revealed $\beta$ values on the order of $1 \AA^{-1}$ for ET rates spanning many orders of magnitude. [179] The observed scatter around a fixed $\beta$ value is an expected consequence of $\beta$ depending on the properties of the intervening tunneling medium. Well-defined molecular donor-bridge-acceptor complexes have also firmly established the sensitivity of ET rates to distance according to equation 5.3. [180]

With this is mind, we set about using measured charge recombination rates in the tunneling regime as a probe of charge pair separations. By rearranging 
equation 5.3, we see that it is not necessary to fully account for absolute ET rates (in this case charge recombination) because the distance distribution is obtained from relative recombination rates for charge pairs in the same material blend;

$$
-\ln \left(\frac{k}{k_{C T}}\right)=\beta\left(R-R_{C T}\right) .
$$

Provided that we resolve a fast recombination component that can be attributed to bound CT states (presumably with an electron-hole separation, $R_{C T} \sim 0.5-1 \mathrm{~nm}$ ), all slower recombination rate constants $(k)$ can be referenced to $k_{C T}$. According to equation 5.4, we can use the observed rates to extract charge pair separation, $R$, relative to the reference distance $R_{C T}$, as depicted in Scheme 5.1. The absolute distance scale is set by the value of $\beta$, which we will return to later.

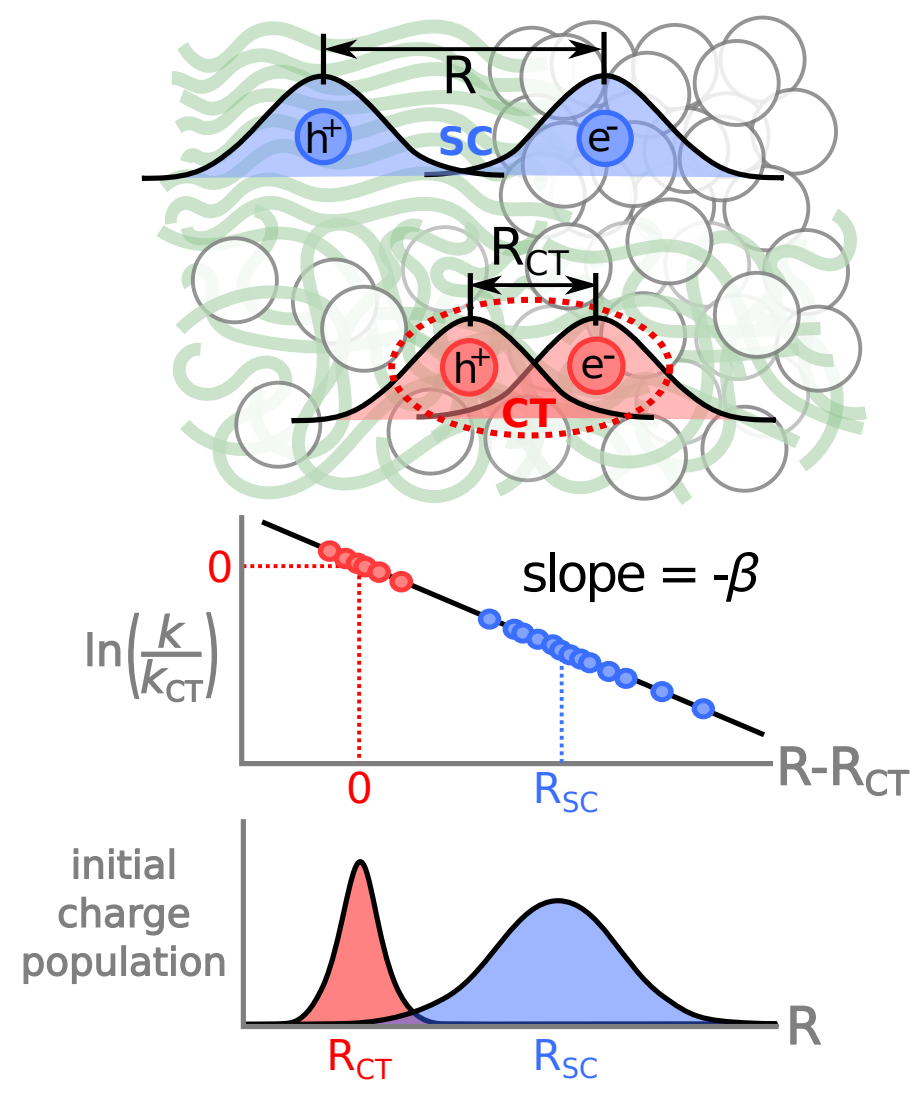

Scheme 5.1: Obtaining charge pair distance from tunneling recombination dynamics.

Figure 5.2 shows the tunneling recombination dynamics for a P3HT:PCBM blend film at $10 \mathrm{~K}$. Although we have accessed a sufficiently low temperature for charge recombination to be predominantly monomolecular, the decay in Figure 
5.2 clearly cannot be fit with a monoexponential or even biexponential decay. Most of the observed recombination is highly dispersive, which is not surprising for a disordered bulk heterojunction blend and reflects a distribution of charge pair separations. We are therefore forced to fit to a distribution of rate constants spanning the wide range of timescales observed.
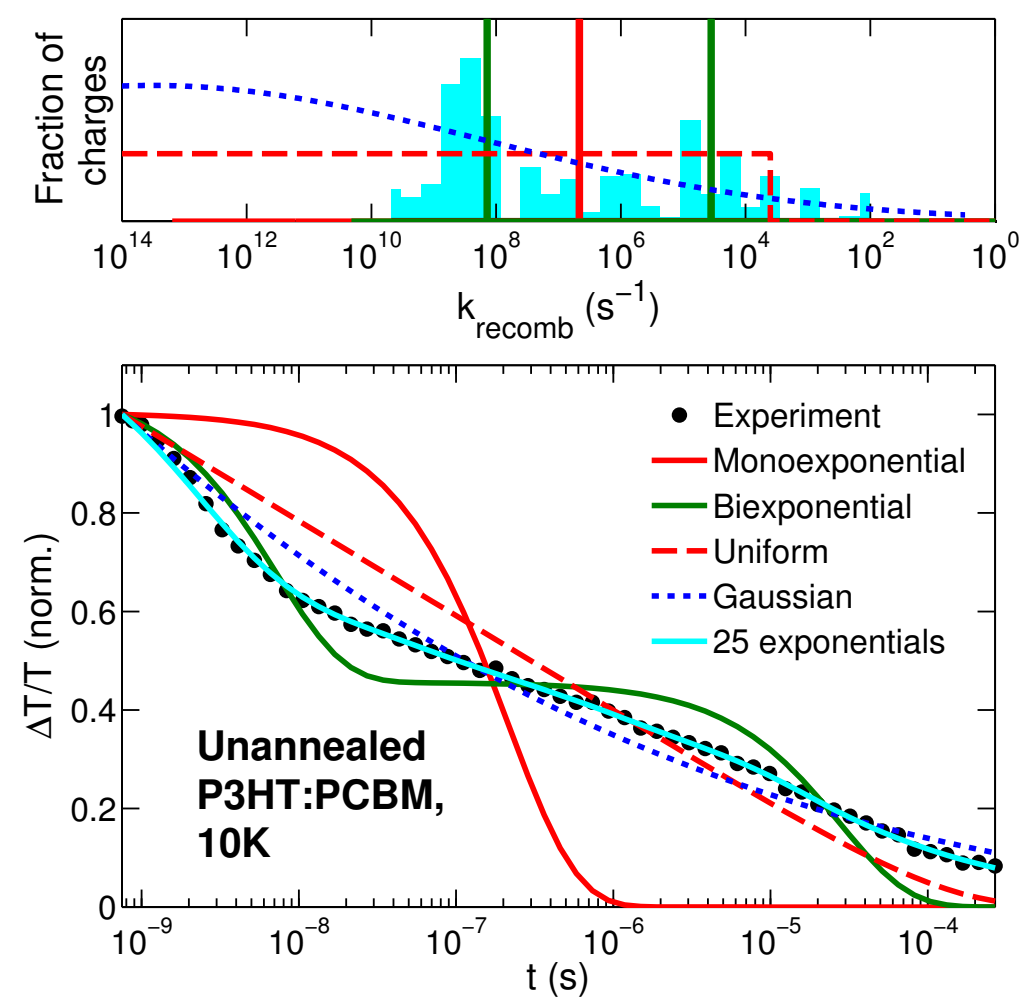

Figure 5.2: $10 \mathrm{~K}$ recombination dynamics for an unannealed P3HT:PC ${ }_{61} \mathrm{BM}$ film fit to various possible distributions of exponential decay functions. The corresponding rate constant distributions are shown in the top panel.

By observing how closely simple distributions of rate constants fit the experimental decay in Figure 5.2, we are guided towards a model that captures the data with minimal free parameters. The charge recombination dynamics appear to be described by a rapid early $(<10 \mathrm{~ns})$ phase that may be fit by a narrow distribution of rate constants, followed by slower dynamics that clearly demand a much broader distribution of rate constants. A uniform series of logarithmically distributed rate constants with fixed equal amplitude predicts a decay that captures the overall dispersion, but fails to capture the apparent faster (CT) decay phase. A broad Gaussian distribution of rate constants leads to a slight improvement, how- 
ever, failure to account for the transition between a fast and slower recombination phase suggests that a bimodal distribution of rate constants is needed. A series of logarithmically distributed rate constants with independent amplitudes fits the data well, but the excess parameters impair quantitative comparison between samples. It can be seen that a bimodal rate distribution emerges after initialization with a flat distribution. Balancing the requirements to fit a bimodal rate distribution with minimal free parameters led us to fit to a rate distribution reflecting a double Gaussian charge pair distance distribution (i.e., double Gaussian on a logarithmic rate scale).

Figure 5.3 confirms that with two Gaussians, we achieve an excellent fit to the experimental data. The fit is not unique; many other bimodal functions could fit the highly dispersive decays equally well, as the unconstrained distribution in Figure 5.2 illustrates. However, the Gaussian model does so with minimal free parameters, from which physically meaningful insights can be derived. The attempted biexponential fit and the 25 independent exponentials fit in Figure 5.2 suggests that the faster phase should fit to a relatively narrow distribution of rate constants, therefore we fixed its width to leave only 4 free parameters (the first four columns of Table 5.1) and ensure a well-anchored component representing the fastest rates that can be defined as the reference $k_{C T}$. The two Gaussian rate constant distributions that best fit the data are peaked at around $3 \times 10^{8} \mathrm{~s}^{-1}$ and $1 \times 10^{5} \mathrm{~s}^{-1}$, integrating to account for $48 \%$ and $52 \%$ of the total charge population, respectively. Our simple model therefore provides a simple measure of the yields of CT states and SCs.

Although the observed bimodal recombination rate distributions may be affected by differences in geometric factors, we consider distance to be of primary importance. In this interpretation, the bimodal rate distribution implies separate populations of closely bound CT states and SCs that would otherwise be free at room temperature. The $\sim 3$ ns lifetime associated with the peak of the fast Gaussian component (defined as $k_{C T}$ ) is in line with expectations for bound CT states in P3HT:PCBM blends, where $2-4$ ns lifetimes are found at room temperature. [102] Secondly, the relative yields of the two populations closely matches the relative yields of bound versus free charges measured independently for the same samples via intensity dependent recombination kinetics at room temperature. Here, we apply the model introduced by Howard et al., [102] whereby the total decay is described by two populations formed via an ultrafast branching step; 

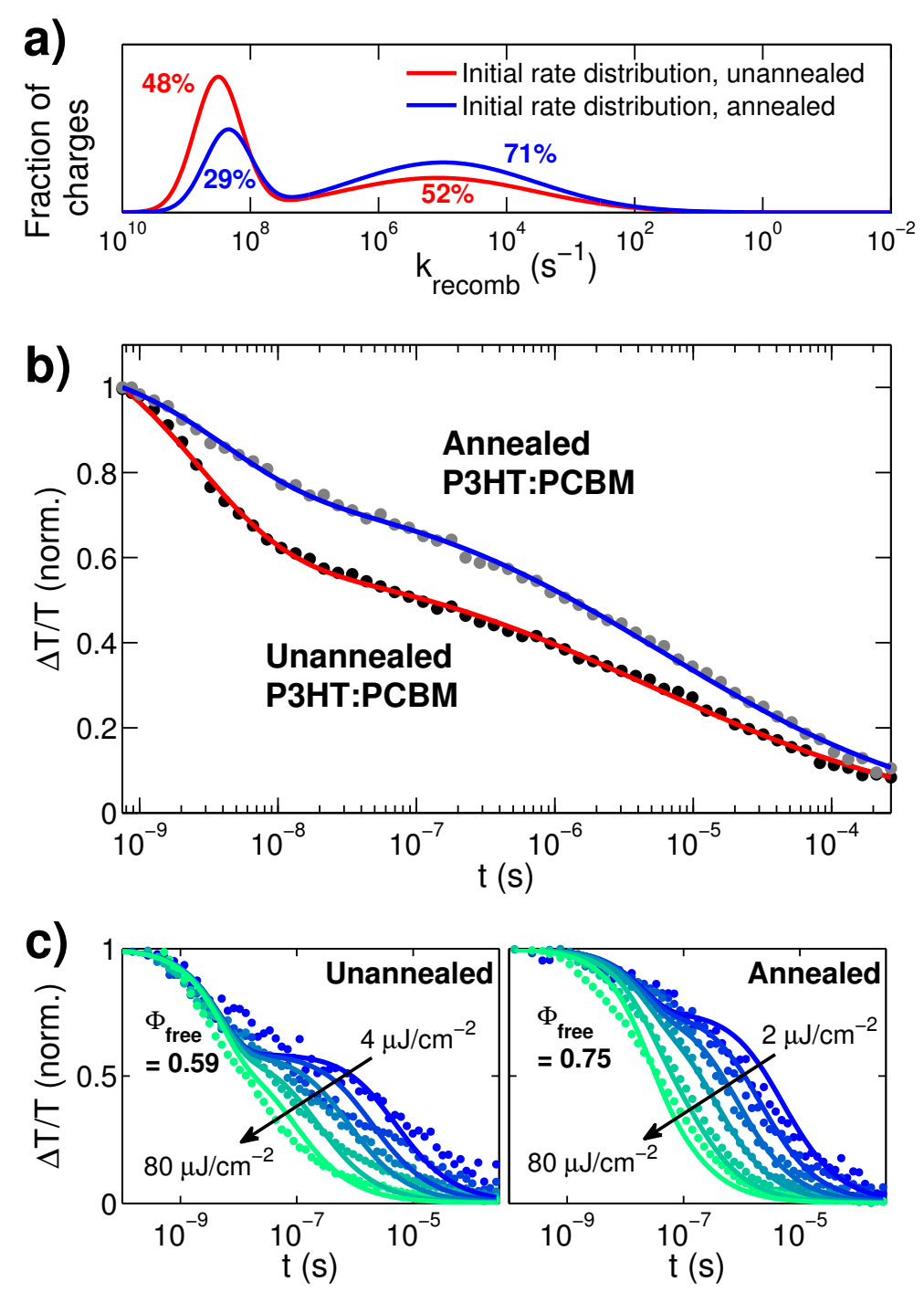

Figure 5.3: a) Distribution of rate constants used to fit recombination decay in b) for annealed and vapor unannealed P3HT:PCBM blend films at $10 \mathrm{~K}$. c) Room temperature intensity dependent recombination for the same films fitted to the kinetic model presented in reference [102]. 
bound CT states recombine monomolecularly, and free charges undergo intensity dependent bimolecular recombination. A common set of four parameters are globally fit across a wide range of intensities, leading to a measure of free charge photogeneration yield that correlates well with device quantum efficiency at room temperature. Figure 5.3c shows the room temperature intensity dependent recombination dynamics for the unannealed P3HT:PCBM film. The fitted $59 \%$ free charge photogeneration efficiency closely matches the $52 \%$ SC yield associated with slower Gaussian component.

In order to test our interpretation that the slow Gaussian component reflects frozen SCs that would otherwise be free at room temperature, we investigated the effect of solvent vapor annealing. Annealing is known to produce blend morphologies that lead to higher free charge yields. [146] Figure 5.3b shows that solvent vapor annealing leads to slower $10 \mathrm{~K}$ charge recombination dynamics. When applying the fit, both Gaussian components undergo negligible shifts in peak rate constants, rather a higher weight (71\%), in the slow Gaussian component accounts for the slower dynamics. The result of the fit is reasonable since the main effect of annealing on the raw recombination data is clearly in the relative weightings of fast $(<10 \mathrm{~ns})$ and slower recombination phases. Again, the relative yield of SCs follows the increased yield of free charges measured via intensity dependent recombination kinetics at room temperature (75\%, Figure 5.3c).

Having established that we are able to identify SCs via their recombination dynamics at $10 \mathrm{~K}$, we applied the procedure to a range of other polymer:fullerene blends of varying efficiency in order to interrogate whether a universal correlation exists. Our survey included low bandgap polymers PCDTBT and PCPDTBT, along with MEH-PPV. We also investigated the effect of blend ratio and excitation wavelength in order to distinguish the role of polymer and fullerene phases in free charge photogeneration. Unless otherwise stated, for each film we measured both recombination dynamics at $10 \mathrm{~K}$, as well as intensity dependent recombination dynamics at $290 \mathrm{~K}$. Each sample was probed at $800 \mathrm{~nm}$, which in most cases corresponds to photoinduced absorption due to the hole polaron population. The exception is the PCPDTBT blend, for which the signal at $800 \mathrm{~nm}$ tracks the groundstate bleach recovery (which is proportional to charge density on the time scales studied here). Broadband TA spectra for each blend can be found in Section 4.3. Particularly fast recombination in MEH-PPV:PCBM blends required the use of a femtosecond excitation source to resolve the signal from $10^{11}-10^{-9} \mathrm{~s}$. Appendix I: 
Early MEH-PPV:PCBM recombination describes how this data was scaled to overlap with the long-delay dataset.

Figure 5.4 shows the set of recombination dynamics at $10 \mathrm{~K}$ along with the fitted distributions of recombination rate constants. The P3HT:PCBM data from Figure 5.3 is repeated here for ease of comparison. Key fitting parameters, along with the yield of free charges independently fitted via intensity dependent recombination at $290 \mathrm{~K}$ (see Section 4.5) are summarized in Table 5.1. Figure 5.4 demonstrates that the double Gaussian model produces excellent fits of the observed dynamics. Alternative unconstrained rate distributions for each sample with 25 independent exponentials (see Appendix II: Complete rate distribution fits) show the emergence of bimodal character, confirming that fitting to a well-parameterized bimodal rate distribution is appropriate. In all cases, the fast phase of recombination equates to bound CT lifetimes peaked at $\sim 2-20 \mathrm{~ns}$ and the slow phases reflect lifetimes peaked at $\sim 1-10 \mu \mathrm{s}$, with substantial width in the slower recombination phase.

\begin{tabular}{|c|c|c|c|c|c|}
\hline & $\begin{array}{c}k_{C T}{ }^{\mathrm{ii}} \\
/ 10^{8} \mathrm{~s}^{-1}\end{array}$ & $\begin{array}{c}k_{S C}{ }^{\mathrm{iii}} \\
/ 10^{4} \mathrm{~s}^{-1}\end{array}$ & $-\ln \left(\frac{k_{S C}}{k_{C T}}\right)$ & $\Phi_{S C}{ }^{\text {iv }}$ & $\Phi_{\text {free }}^{\mathrm{v}}$ \\
\hline Unannealed P3HT:PC ${ }_{61} \mathrm{BM}$ & 3.2 & 12 & 7.9 & 0.52 & 0.59 \\
\hline Annealed P3HT:PC ${ }_{61} \mathrm{BM}$ & 2.2 & 9.8 & 7.7 & 0.71 & 0.75 \\
\hline P3HT:PC ${ }_{61}$ BM (19:1) & 1.5 & 3.6 & 8.4 & 0.8 & - \\
\hline P3HT:PC ${ }_{61} \mathrm{BM}(355 \mathrm{~nm})$ & 2.3 & 3.7 & 8.7 & 0.58 & 0.71 \\
\hline PCDTBT:PC ${ }_{61} \mathrm{BM}$ & 0.5 & 7.4 & 6.5 & 0.73 & 0.72 \\
\hline PCDTBT:PC ${ }_{61}$ BM (19:1) & 0.8 & 5.8 & 7.2 & 0.87 & - \\
\hline PCPDTBT: PC $_{61} \mathrm{BM}$ & 3.5 & 86 & 6 & 0.54 & 0.64 \\
\hline MEH-PPV: PC $_{61}$ BM $(1: 1)$ & 7.5 & 14 & 8.6 & 0.2 & 0.26 \\
\hline MEH-PPV: PC $_{61}$ BM (1:4) & 7.3 & 14 & 8.6 & 0.28 & 0.41 \\
\hline
\end{tabular}

Table 5.1: Fitting parameters for charge recombination measurements in different blend films.

Figure 5.5a shows that the yield of SCs (from the slow tunneling phase) is very strongly correlated with the yield of free charges extracted from intensity dependent measurements across the series of samples with widely varying efficiencies. The least squares regression line has a slope close to 1 , and an intercept close to 0 , which is strong evidence that our analysis of the two independent measurements 

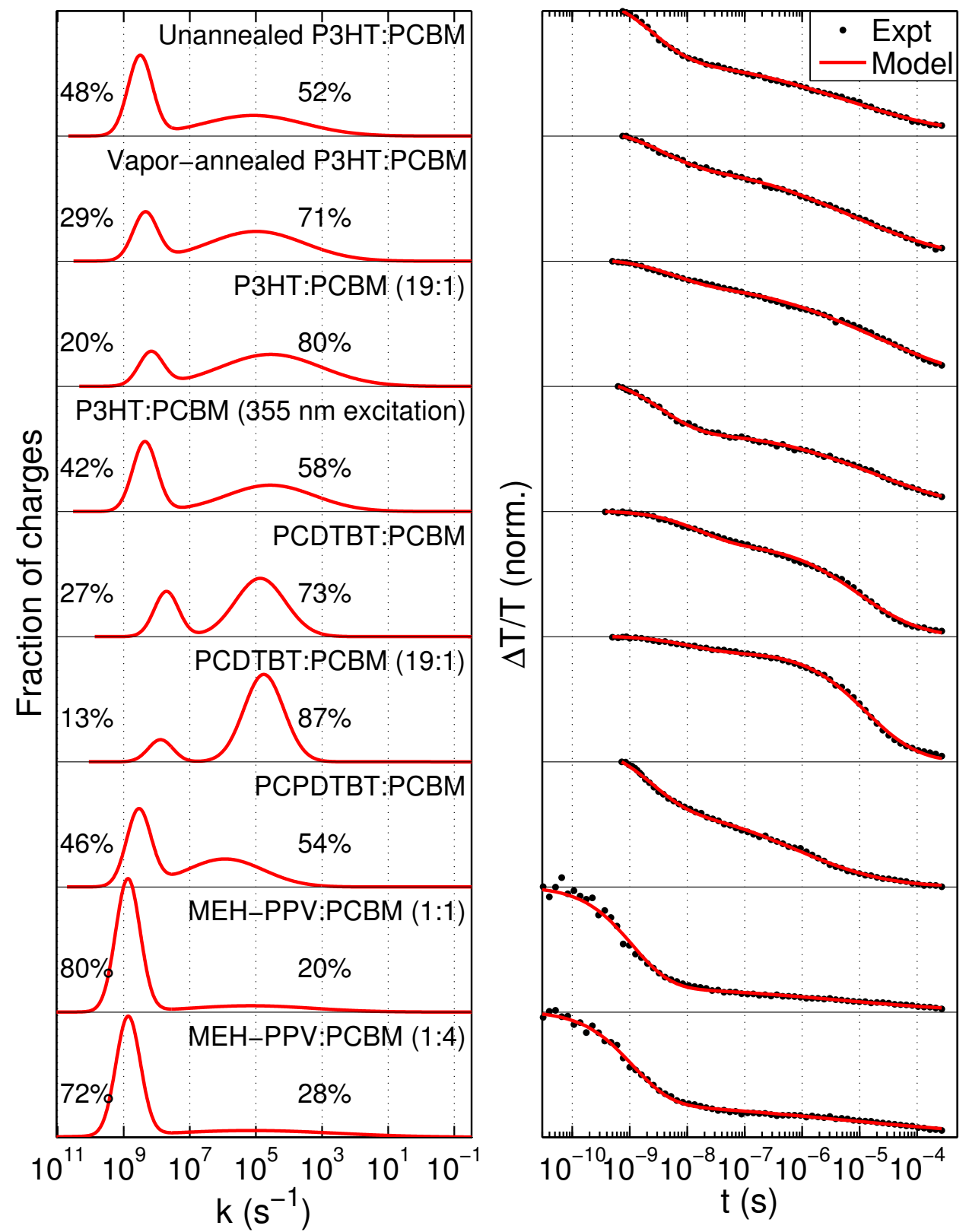

Figure 5.4: $10 \mathrm{~K}$ charge recombination dynamics (right) for various blend films and the corresponding distributions of rate constants (left) used to fit the decays. The relative yields contained in each Gaussian component are indicated on the plots. Alternative unconstrained rate distributions for each sample are provided in Appendix II: Complete rate distribution fits. Unless otherwise stated, samples were photoexcited at $532 \mathrm{~nm}\left(\sim 40 \mu \mathrm{J} \mathrm{cm}^{-2}\right)$ and probed at $800 \mathrm{~nm}$. For data at short time delay $\left(10^{11}-10^{-9} \mathrm{~s}\right)$, MEH-PPV:PCBM was photoexcited at $532 \mathrm{~nm}\left(12 \mu \mathrm{J} \mathrm{cm}{ }^{-2}\right.$, $100 \mathrm{fs})$. 
is sufficiently well parameterized to derive insight into the properties of SCs. Yields of SCs / free charges (measured via both methods) are highest for blends of PC61BM with either P3HT, PCPDTBT, or PCDTBT, followed by the unannealed P3HT blend, while MEH-PPV blends have the lowest free charge yields. This trend broadly correlates with photon-to-current quantum efficiencies previously measured for devices made from the same materials. [57,60,146, 149, 165]

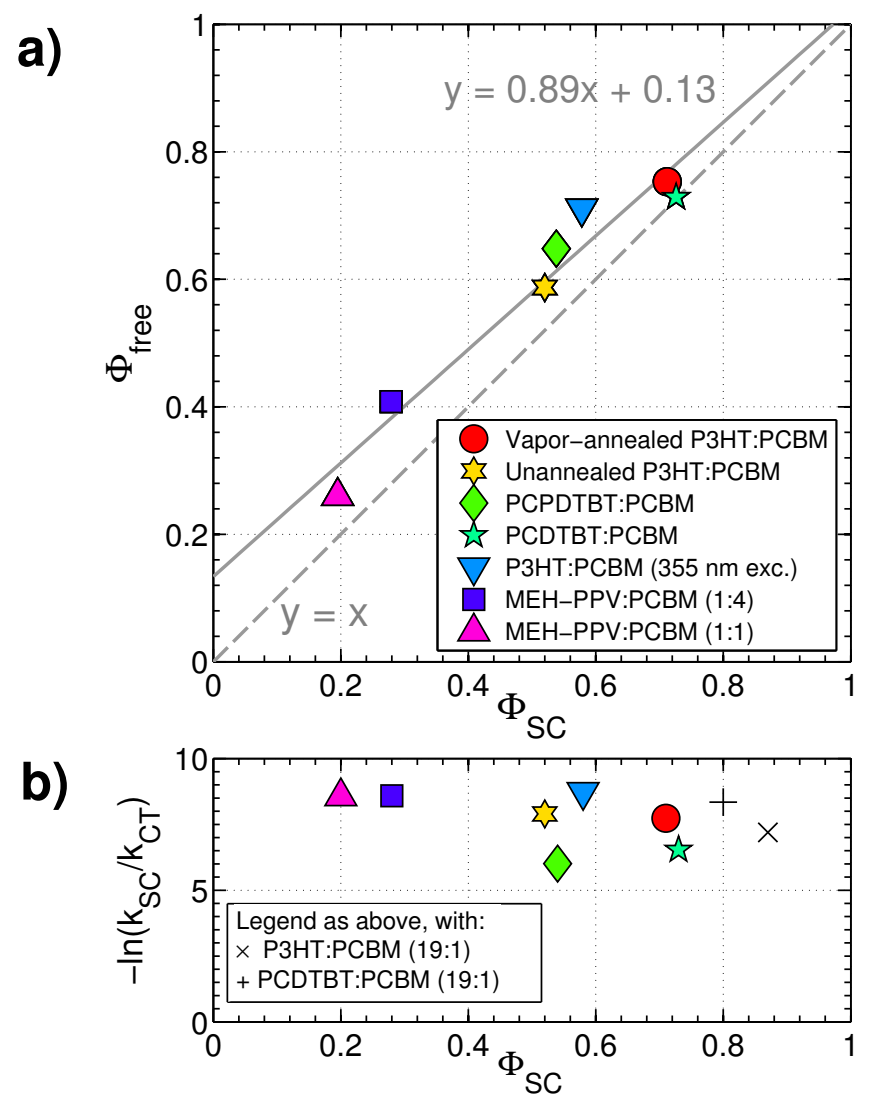

Figure 5.5: Yield of SCs at $10 \mathrm{~K}$ (from the slower fitted Gaussian component) versus the yield of free charges measured via fitting the intensity dependent recombination at room temperature to the kinetic model in reference [102]. The linear regression fit is also shown, along with the $y=x$ line as a guide. The full set of intensity dependent recombination fits can be found in Section 4.5.

Before we consider the question of absolute distances, we can compare the relative charge separation distributions across the series of materials on the basis that $-\ln \left(k_{S C} / k_{C T}\right)$ is proportional to charge pair separation (Eq 5.4), where $k_{S C}$ is the peak rate for SCs. Since each value of $k_{S C}$ is referenced to the $k_{C T}$ value measured for the same sample, the minor intrinsic rate variations between different 
materials is already accounted for (e.g., non-distance contributions to electronic coupling). These values are plotted in Figure 5.5b. Considering the wide range of charge separation efficiencies probed, overall we observe very little variation in $-\ln \left(k_{S C} / k_{C T}\right)$; the total range is only $35 \%$ of the mean value. This shows that SCs for different materials are all described by similar critical separations (assuming similar $\beta$ values); variation in recombination dynamics reflects the relative probability that charge pairs achieved the critical separation for different materials.

By comparing selected pairs of samples, we can also look deeper within the universal correlation to identify the underlying reasons for variation. While comparing the efficiency of different blends is not a new strategy, we emphasize that here we are isolating the properties of the frozen primary charge pairs. In the case of the 1:1 P3HT:PCBM blends, we see that solvent vapor annealing translates to higher yields of SCs (vide supra) as a result of forming more crystalline phases of an optimum size.

In the case of MEH-PPV:PCBM blends, molecular PCBM is more readily dissolved throughout MEH-PPV since MEH-PPV is less crystalline than P3HT. Accordingly, optimized MEH-PPV:PCBM devices feature excess PCBM in order to create sufficiently large and interconnected PCBM phases. Although it is reasonable to assume that the need for excess PCBM relates to electron extraction, our comparison of 1:1 and 1:4 blends shows that excess PCBM results in higher yields of primary SCs. Consistent with a recent combined theoretical and experimental study by Savoie et al., [104] the difference between the relative distance distributions for the MEH-PPV blends reflects the benefit of larger PCBM domains in the charge separation step.

By exciting the P3HT:PCBM blends at $355 \mathrm{~nm}$ as well as $532 \mathrm{~nm}$, we were able to observe that direct PCBM excitation can also lead to comparably high SC yields. This result is consistent with other recent spectroscopic measurements [181] and spectrally resolved photocurrent measurements for devices at room temperature.

We also probed the relative roles of polymer and fullerene components in free charge photogeneration by carrying out measurements on films with only $5 \%$ PCBM blended with P3HT or PCDTBT (labeled as 19:1). The low PCBM content remains sufficient to achieve efficient initial charge photogeneration, [91] however the small and isolated PCBM domains cannot play a significant role in creating SCs. When comparing the 19:1 blends with more fullerene-rich counterparts, 
surprisingly, we find that the creation of SCs is not suppressed by PCBM dilution. In fact, we observe a higher relative yield of SCs (compared with CTs) in the 19:1 blends for both P3HT and PCDTBT, noting that the total charge yield could be diminished due to exciton decay beforehand. This result does not contradict the established higher optimum PCBM content in devices because free charge photogeneration must be balanced with charge extraction requirements in devices. Limited electron diffusion in the 19:1 blends also means that free charge formation cannot be alternatively quantified via intensity dependent recombination dynamics, [102] or by pump-push-probe photocurrent spectroscopy. [100] The tunneling recombination measurement is uniquely positioned to examine the intrinsic charge separation step in these dilute blends, and leads us to conclude that formation of SCs does not necessarily require large PCBM phases. Unlike the MEH-PPV blends discussed above, where PCBM crystals were crucial for charge separation, the blends with only 5\% PCBM highlight the role of the polymers in achieving initial long-range charge separation. The higher yield of SCs for 19:1 blends may be attributed to more extensive and ordered pure polymer domains. Extended polymer chains support highly delocalized excitons, which may couple to SC states prior to localizing. [98]

Finally, we address the question of absolute length scales by considering the tunneling attenuation factor, $\beta$. Most commonly formulated in a superexchange model, lower values of $\beta$ result when the tunneling barrier is reduced. [179,180,182, 183] In donor-bridge-acceptor model systems, $\beta$ values as low as $\sim 0.1 \AA^{-1}$ have been measured when $\pi$-conjugated bridges with a small energy gap are used, thus contributing to electron tunneling over distances longer than $3 \mathrm{~nm}$. [180] $\beta$ values smaller than $0.6 \AA^{-1}$ are considered reasonable for OPV blends, which inherently feature a dense set of low energy gap $\pi$-conjugated tunneling pathways. [108] We note that $\beta$ for more commonly studied charge separation reactions is not the same as for charge recombination in the same photoinduced ET system; the former couples an excited state with a charge-separated state, while the latter returns the charge-separated state to the electronic ground state. Slightly higher $\beta$ values have been measured for charge recombination in some donor-bridge-acceptor systems, [180] and it is tempting to assume that this must always be the case on account of the electron stabilization in the charge-separated state contributing to a higher tunneling barrier for charge recombination. However, it is important to realize that in the formalism of equation 5.1, electron tunneling is in fact dependent 
on the tunneling of nuclear coordinates. Since recombination couples chargeseparated and ground-state surfaces (without involvement of the excited state that preceded charge separation), low $\beta$ values for charge recombination in OPV films are still considered likely.
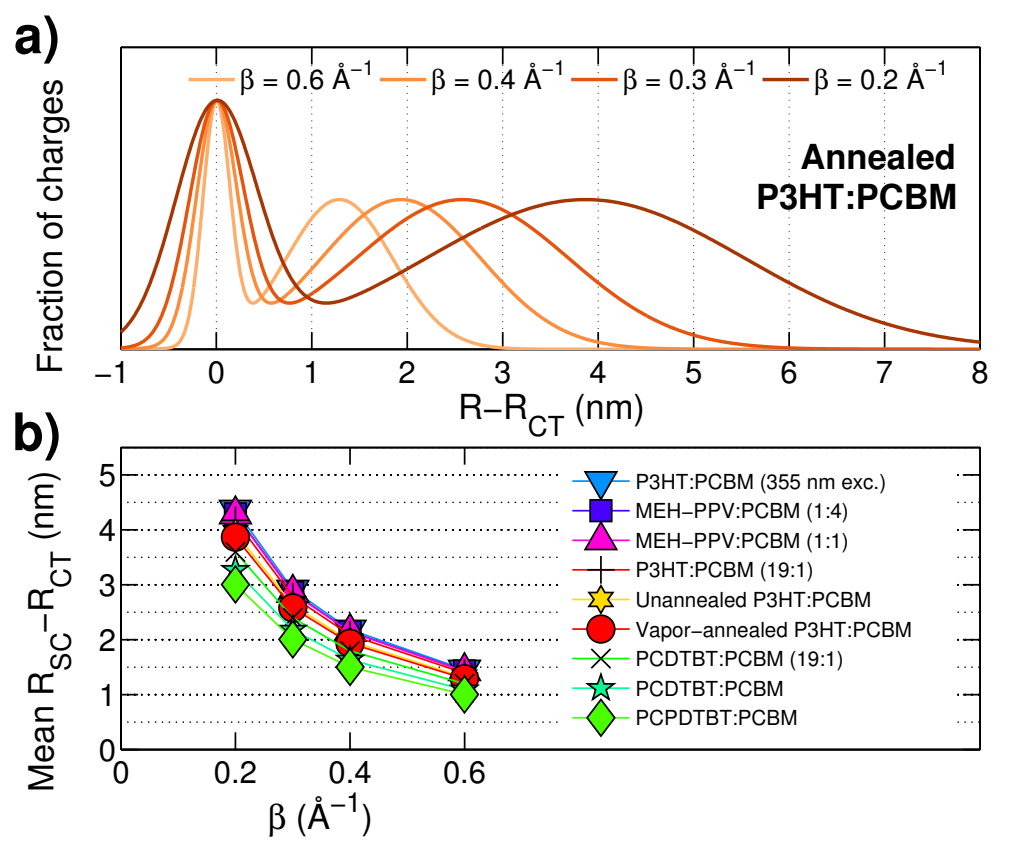

Figure 5.6: a) Extracted distributions of charge pair distances for annealed P3HT:PCBM obtained by applying various values of $\beta$ to the fitted tunneling rate constant distribution in Figure 5.3. b) Mean charge pair distances as a function of $\beta$ for the SC (slower recombining) populations of charges fitted for various blends in Figure 5.4.

Caruso and Troisi calculated distributions of $\beta$ for charge separation by modeling entire disordered polymer-fullerene heterojunctions over several nanometers. [108] They found broad distributions of $\beta \sim 0.2-0.6 \AA^{-1}$. The observed range reflects the dependence on energetic disorder and the anisotropy. In the absence of a similar detailed study on $\beta$ for charge recombination in the materials studied here, we apply this reasonable range of $\beta$ values to transform our measured rate distributions into distance distributions.

Figure 5.6a shows the distance distribution of charge pairs for vapor annealed P3HT:PCBM. The plot is obtained by using Equation 5.4 to transform the recombination rate constant distributions in Figure 5.3 onto a distance scale, using several plausible $\beta$ values. $k_{C T}$ is taken as the peak of the fast recombination phase, such 
that the $x$-axis represents the excess charge separation compared with CT states. For $\beta \sim 0.3-0.2 \AA^{-1}$, the SCs have separations $\sim 2.5-4 \mathrm{~nm}$ greater than CT states.

Figure $5.6 \mathrm{~b}$ shows the distance corresponding to the peak of the SC population for all films as a function of $\beta$. The values were obtained by applying Equation 5.4 to the ratio $k_{S C} / k_{C T}$ taken from in Table 5.1, which were derived from the fitted charge recombination curves in Figure 5.4. The tight clustering of the charge separation values reflects the similarity of the rate constants noted above and in Figure 5.5b. Since the SC yields were already shown to be correlated with free charge yields (Figure 5.5), the SC distances shown in Figure 5.6b represent critical charge separations that must be achieved within the thermalization timescale in order to generate free charges. Figure $5.6 \mathrm{~b}$ shows that reasonably low values of $\beta$ $\left(0.3 \AA^{-1}\right)$ consistently place the SC distances $\sim 2-3 \mathrm{~nm}$ greater than for CT states. Even the maximum $\beta$ value of $0.6 \AA^{-1}$ places SC distances $\sim 1.5 \mathrm{~nm}$ greater than for CT states. Considering the range of measured $\ln \left(k_{S C} / k_{C T}\right)$ values, of possible $\beta$ values and of CT separations, we conclude that free charges are defined by primary thermalized separations of $\sim 3-4 \mathrm{~nm}$. The absolute distance scale could be further refined by calculating distributions of $\beta$ for charge recombination in these materials (along the lines of reference [108]) and applying to the measured rate distributions.

\subsection{Conclusions}

We were able to access a regime at low temperature where photogenerated charge pairs are frozen at the separations at which they thermalize and slowly recombine via tunneling. By fitting tunneling recombination dynamics to a double Gaussian distribution of rate constants, we identified populations of CT states and SCs. We observed a universal link between the yield of initial SCs at low temperature and the yield of free charges identified by bimolecular charge recombination at room temperature. We therefore conclude that in OPV devices, populations of free (extractable) charges are established via long-range charge separation within the thermalization timescale. Subject to assumed values of $\beta \sim 0.3 \AA^{-1}$, we find that initial charge separations of $\sim 3-4 \mathrm{~nm}$ equate to free charges at room temperature in all material blends, and differences between materials are manifest in their branching ratios. Our measurements support the various branching models and augment them with spatial information. The bimodal recombination rate 
distribution, which also emerges from fits that do not presume its shape, provides evidence of an energetic barrier between populations of CTs and SCs.

Because we recover information about primary charge pairs by freezing them and resolving their subsequent recombination, we are unable to time-resolve the distance coordinate of charge separation. Thus, we cannot distinguish between direct long-range charge separation (perhaps aided by delocalization) and rapid charge diffusion during thermalization. Nevertheless, the experiment and analysis we have developed provides an insightful probe of the properties of initial charge pairs. By resolving the effect of fullerene content in MEH-PPV blends, we found that charge separation yields were dependent on forming fullerene crystals. However, the important role of the polymers was highlighted in blends that achieved significant charge separation with minimal fullerene. We found that SCs were also generated via fullerene photoexcitation. We expect the approach described to be of considerable value in guiding new material development and may form the basis of correlations against, for example disorder parameters, CT excitation energy, quantum coherence, or delocalization lengths. By isolating distance distributions achieved in the primary charge separation step, tunneling recombination measurements are ideally positioned to test the validity of current and future models for long-range charge separation.

\subsection{Appendix I: Early MEH-PPV:PCBM recombination}

An appreciable fraction of photogenerated charge pairs within MEH-PPV:PCBM blends undergo recombination in less than $1 \mathrm{~ns}$, faster than the time resolution of our TA experiment when using the electronically delayed excitation source. Additional data was therefore collected using a $100 \mathrm{fs}$ excitation pulse and mechanical delay stage, capable of collecting data up to a delay of several nanoseconds. Data from the two time ranges was normalized so as to coincide from 1-2 ns.

The combined datasets are shown in Figures 5.7 and 5.8. Also shown is the relative amplitude of the fs and ps peaks, which is used to correct the free charge fraction fitted from intensity-dependent recombination measurements (see Section 4.5).

The collected data represents the convolution of our instrument response function $R(t)$ and the true signal decay, $S(t)$. This convolution is given by $I(t)=$ $\int R\left(t-t^{\prime}\right) S(t) d t^{\prime}$. 

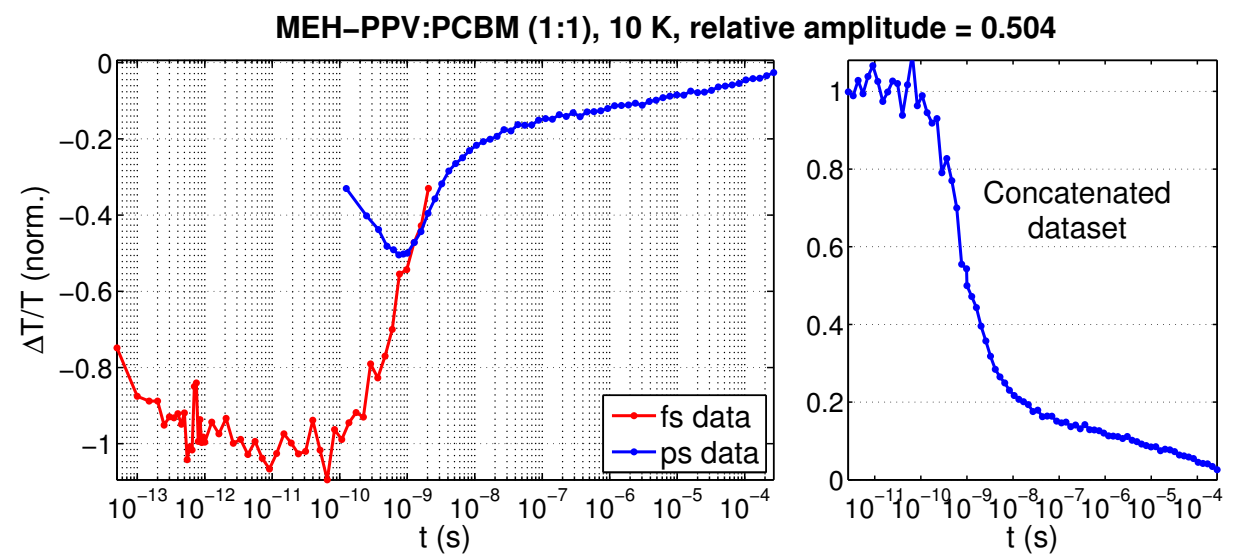

Figure 5.7: Combined fs pump and ps pump kinetics for MEH-PPV:PCBM (1:1)
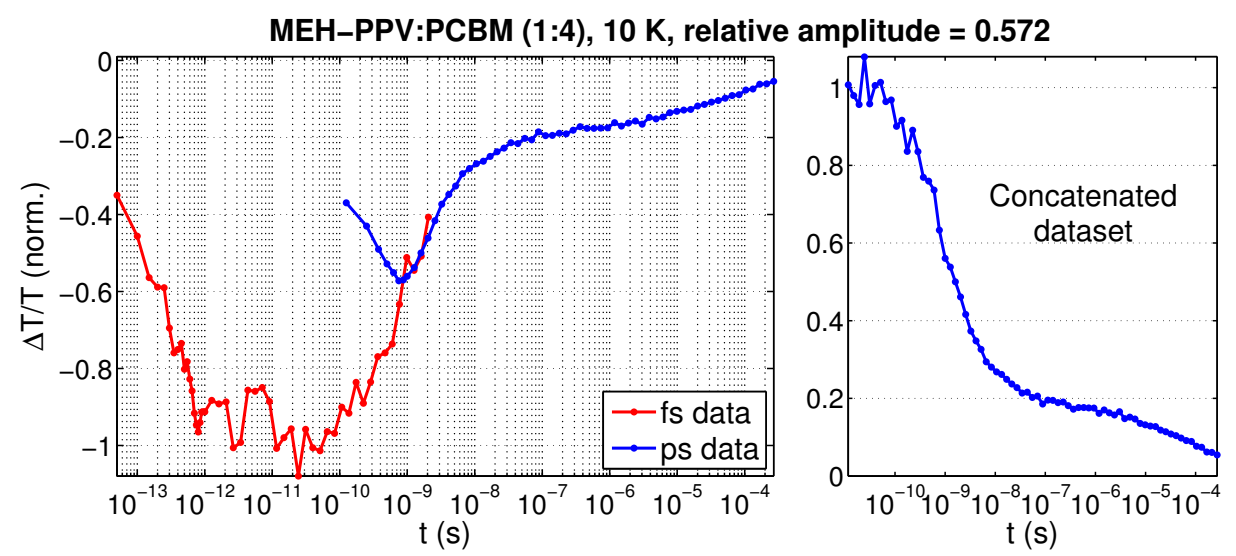

Figure 5.8: Combined fs pump and ps pump kinetics for MEH-PPV:PCBM (1:4)

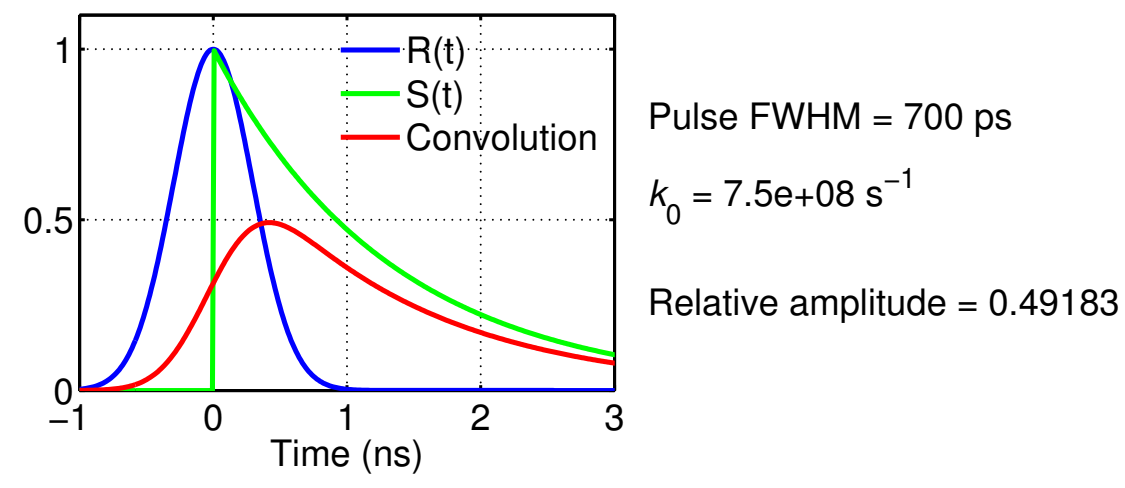

Figure 5.9: Numerically calculated convoluted measurement of signal and instrument response 
If we take $R(t)$ as a Gaussian pulse with FWHM $=700 \mathrm{ps}$, and $S(t)$ as a monoexponential with rate $=7.5 \times 10^{8} \mathrm{~s}^{-1}$, a simple numerical calculation of the convolution function predicts a relative amplitude of 0.49 , in agreement with the concatenated datasets shown in Figure 5.9.

\subsection{Appendix II: Complete rate distribution fits}

The complete plots and fitted parameters for the data shown in Figure 5.4 of the main text are shown below (left side). The width of the bound charge pair distribution was fixed, leaving four fitting parameters

The fitting routine proved to be exceptionally robust, with the same fitted parameters being returned for a wide range of initial conditions. Fits for all materials were calculated from identical initial conditions.

While the bimodal Gaussian distribution generates time-dependent recombination in excellent agreement with experiment, it is not a unique solution. One alternative is to define an ensemble of independent charge-pair populations, each with a well-defined monoexponential decay rate. Compared to the double-Gaussian fits, this approach is poorly parameterized and does not lend itself to physical interpretation. However, it has the advantage of being without bias in the distribution of rates (i.e. it does not presume a bimodal distribution).

The fits shown below (right side) feature 25 rates logarithmically spaced from $\sim 10^{10}$ to $10^{2} \mathrm{~s}^{-1}$. The fitted parameters are the 25 initial populations for each rate, with the initial condition being an equal distribution of charges across all rates.

While the approach is not always successful, in most cases we see the emergence of a (somewhat noisy) bimodal distribution with an inter-peak minima near $10^{7} \mathrm{~s}^{-1}$ and a population distribution resembling those given by the double Gaussian fits. 


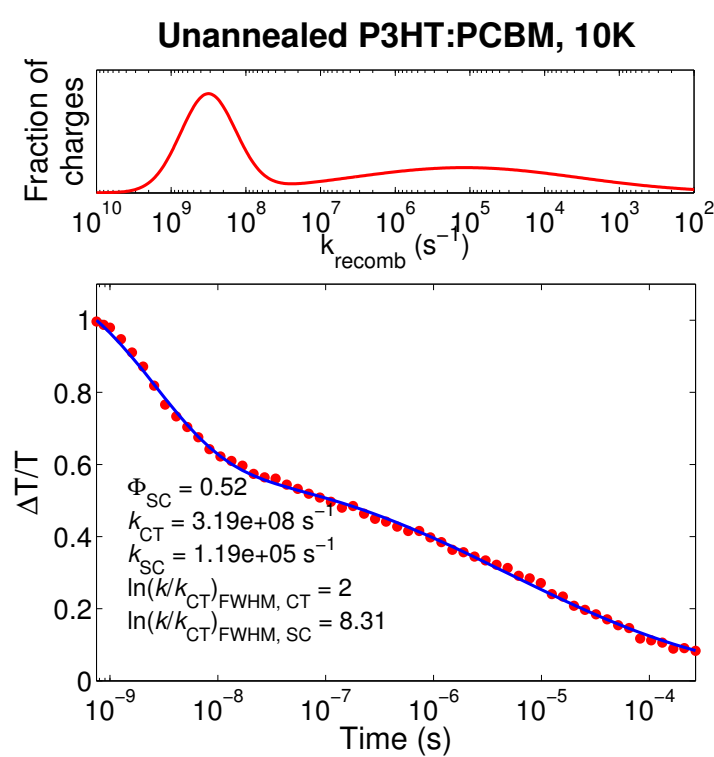

(a)
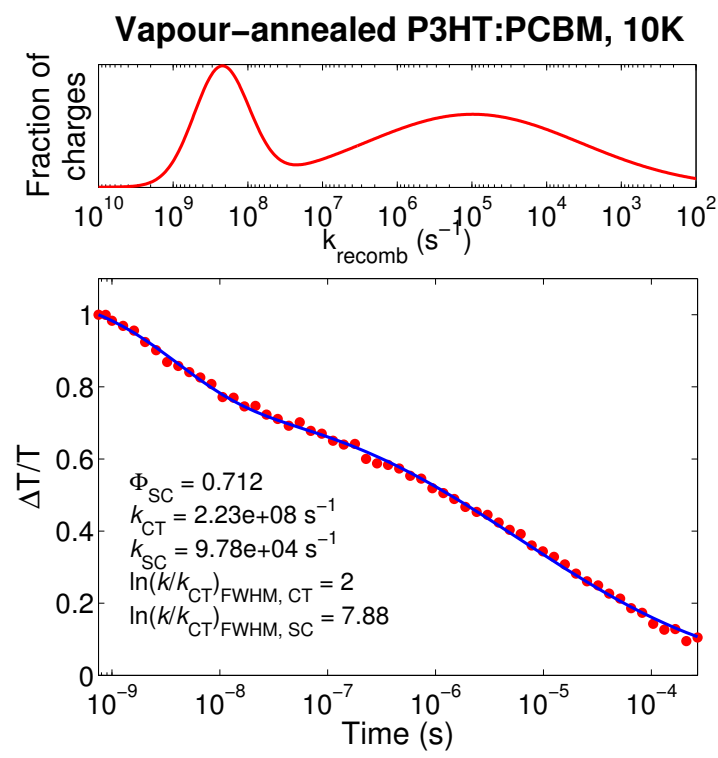

(c)
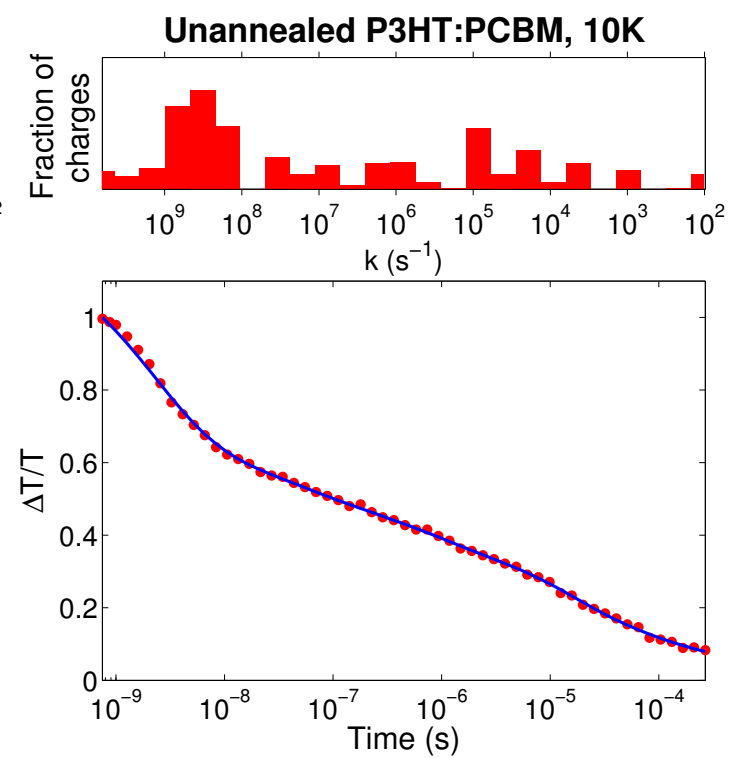

(b)
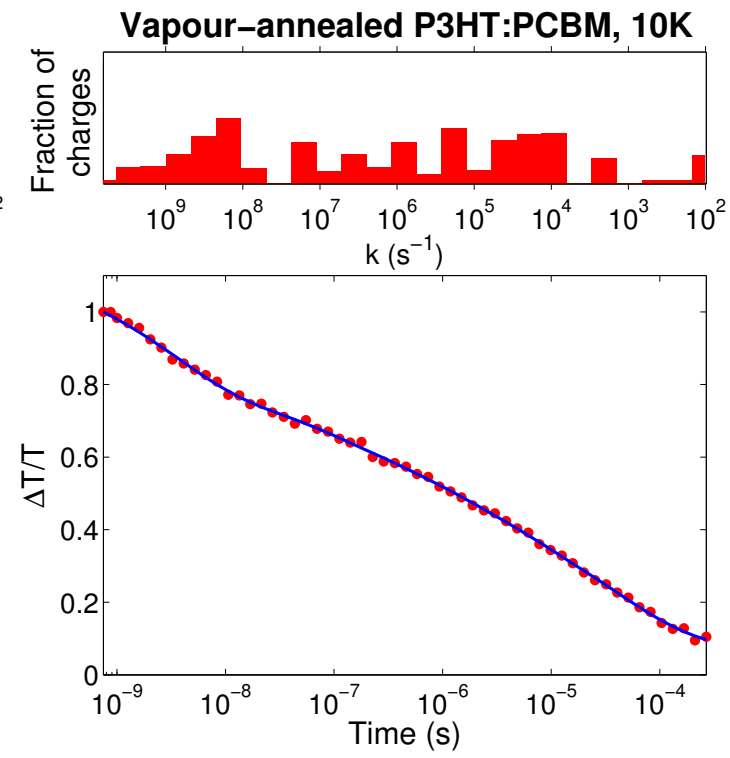

(d) 

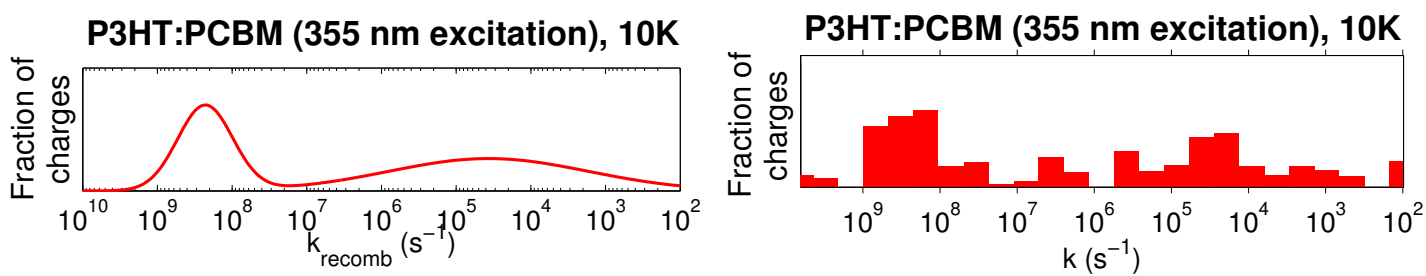

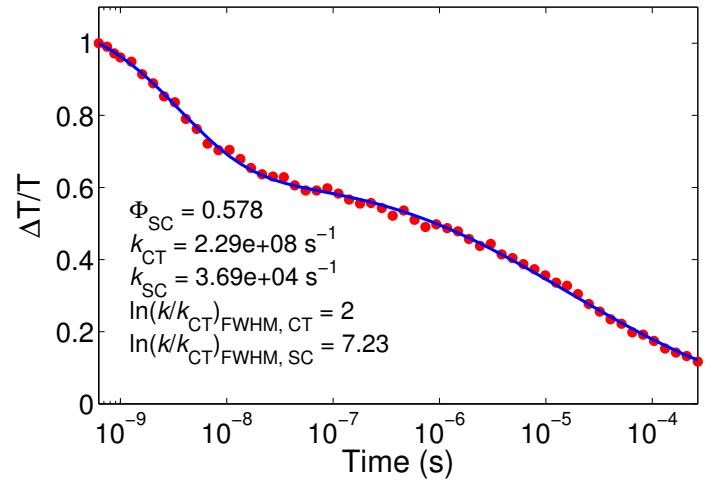

(e)
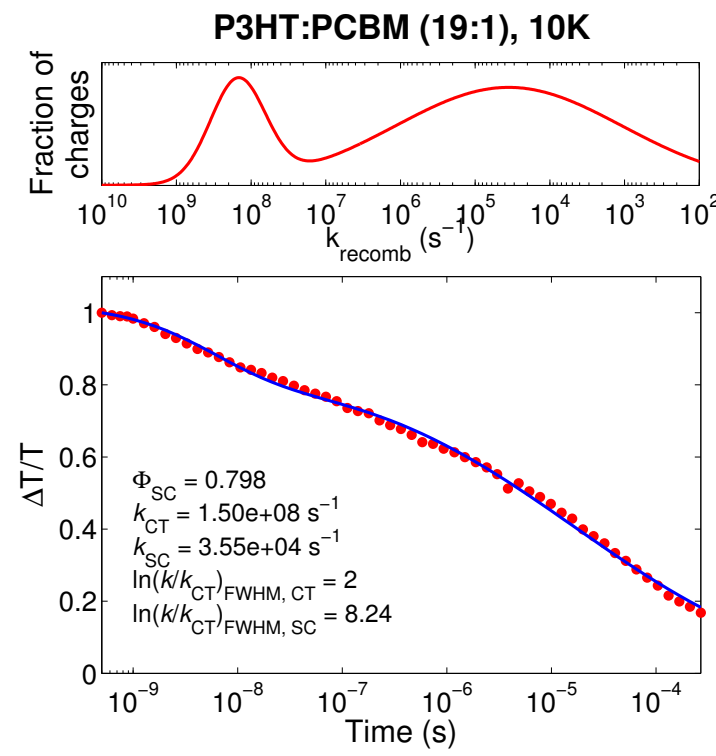

(g)

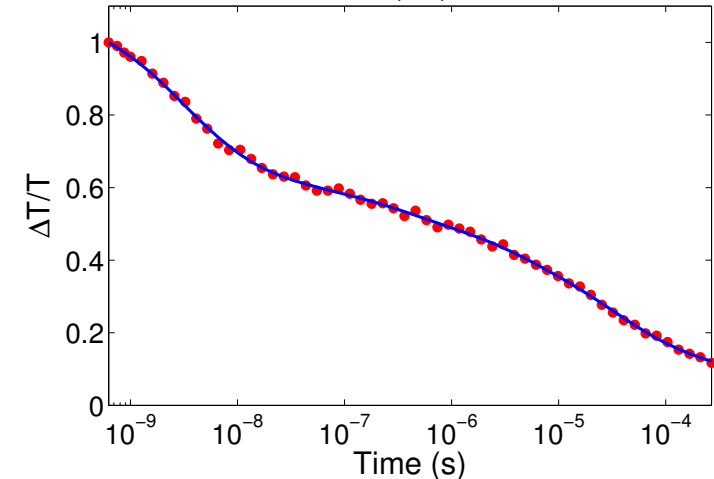

(f)
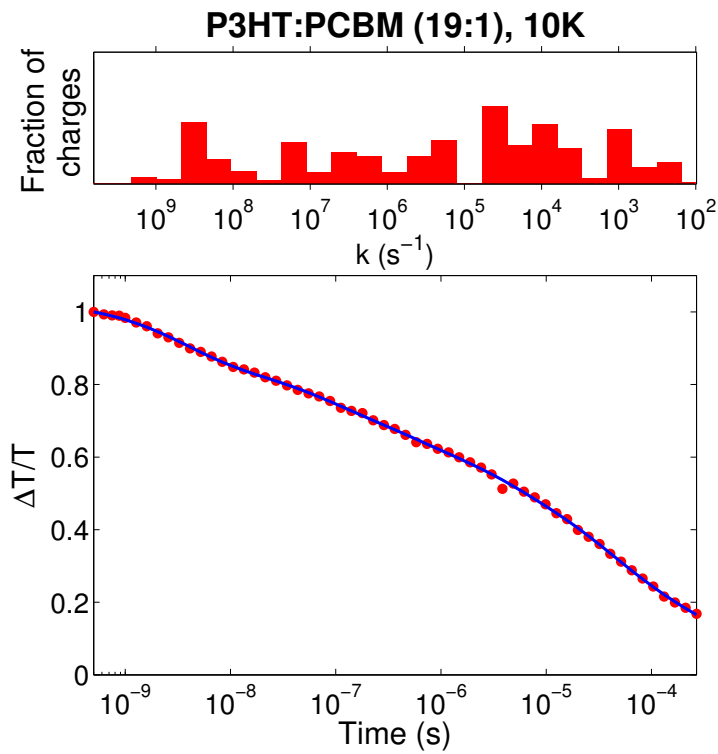

(h) 

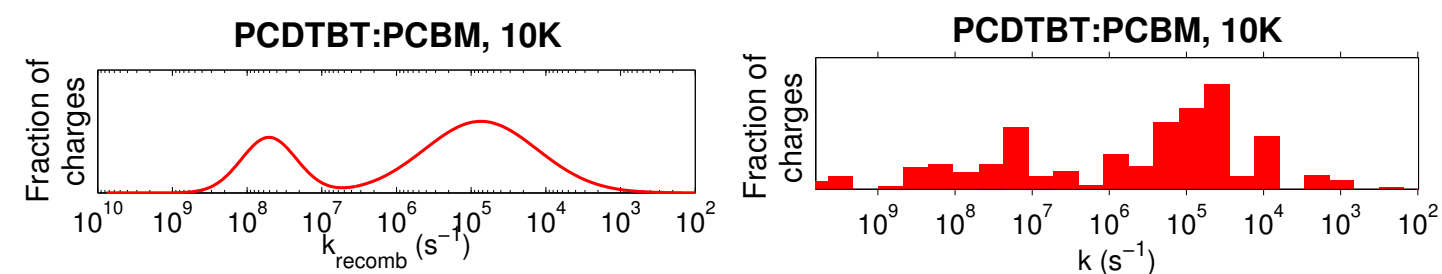

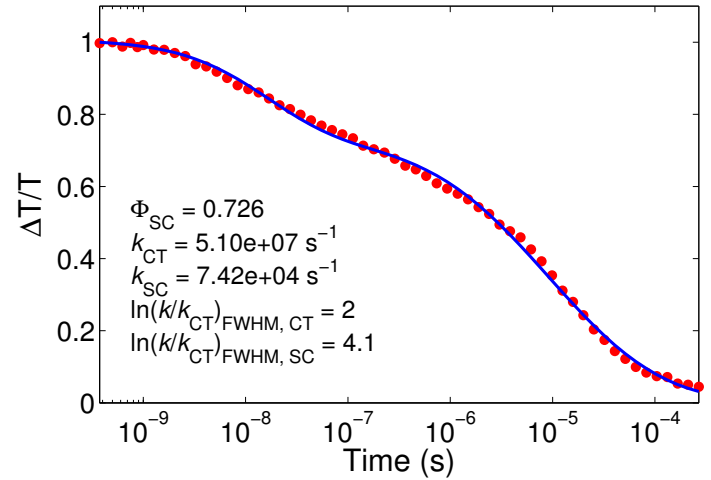

(i)
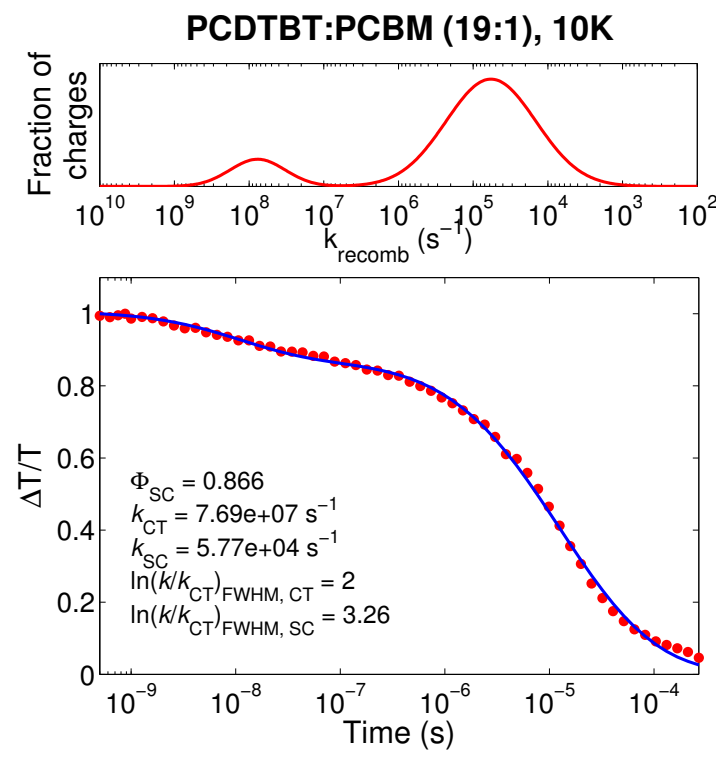

(k)

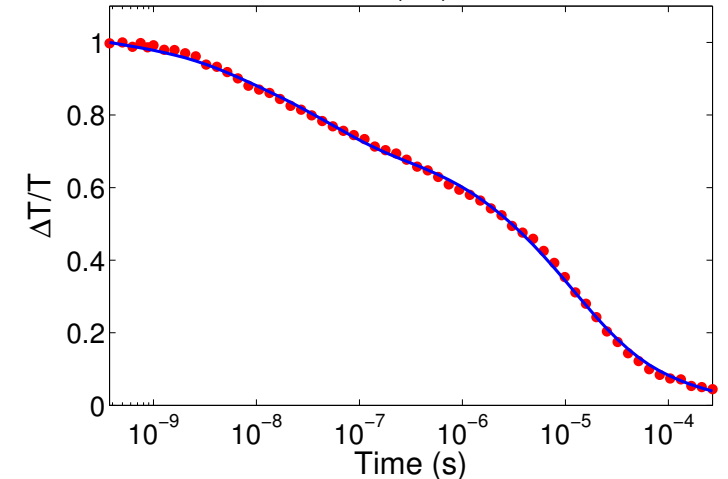

(j)
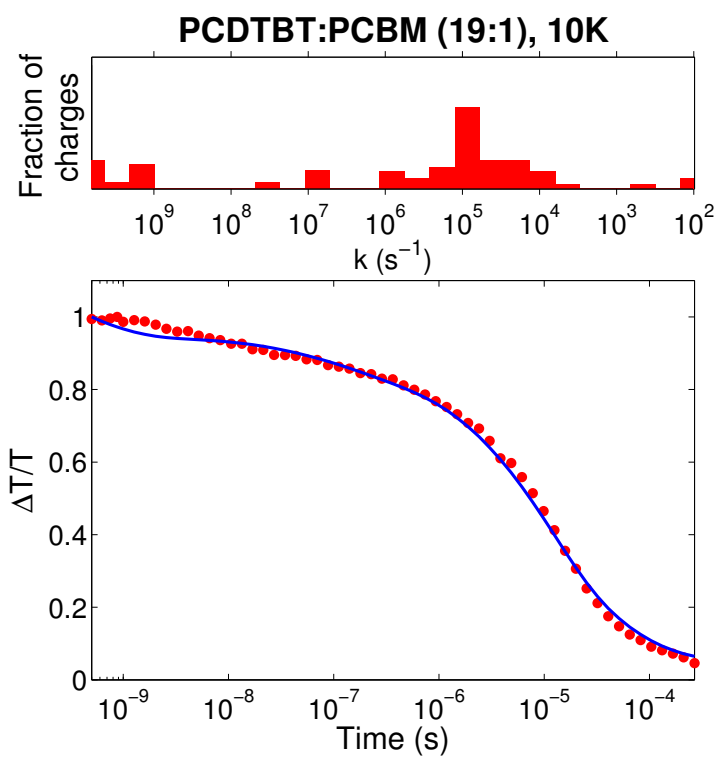

(l) 

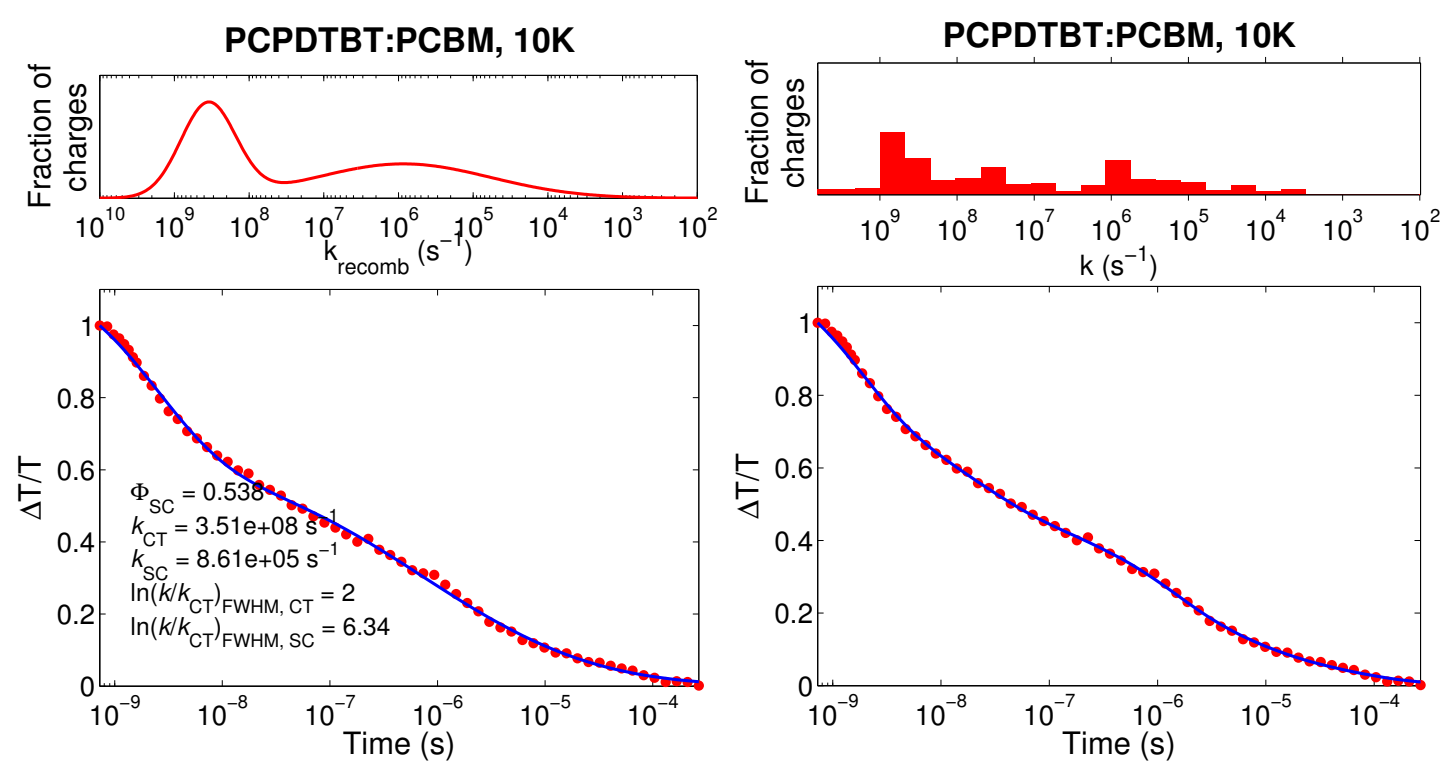

(m)

(n)
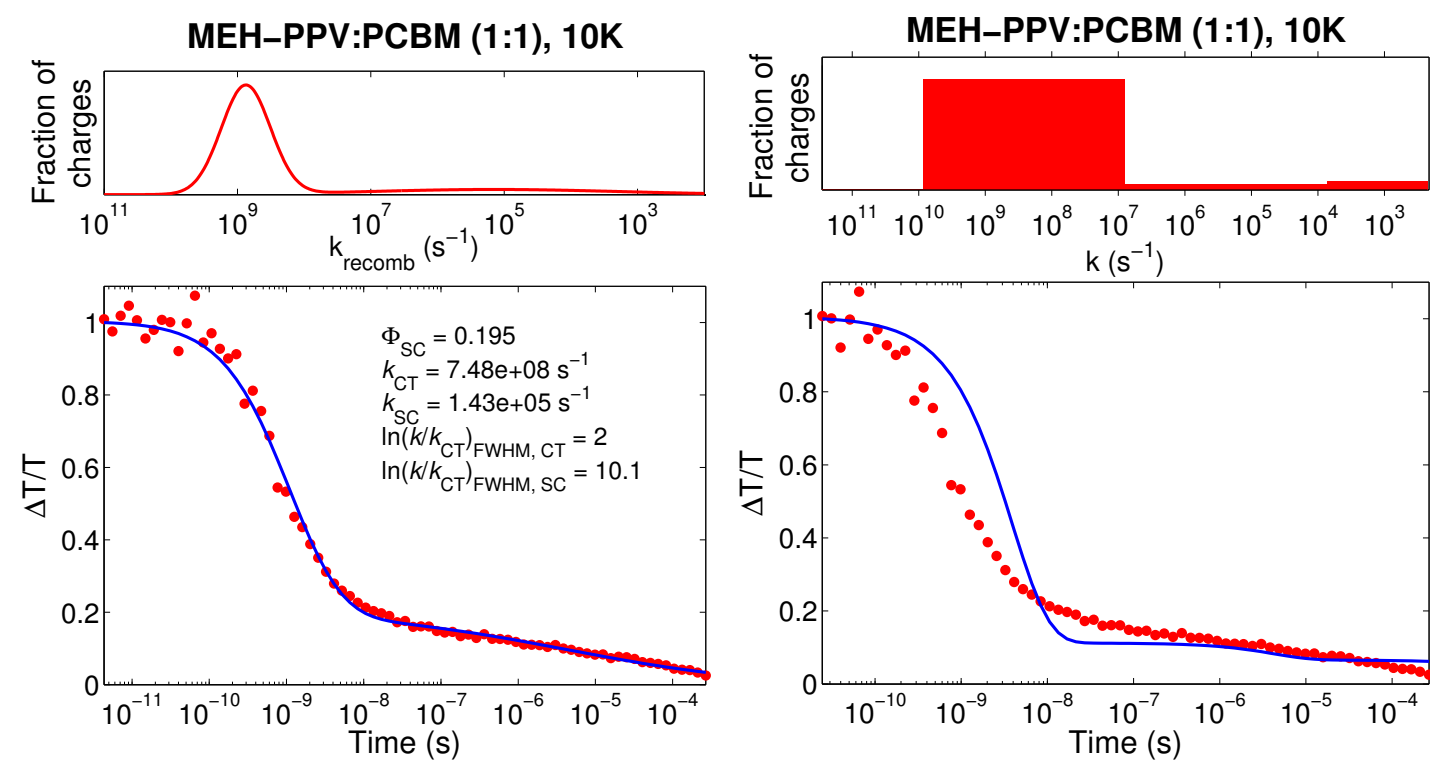

(o)

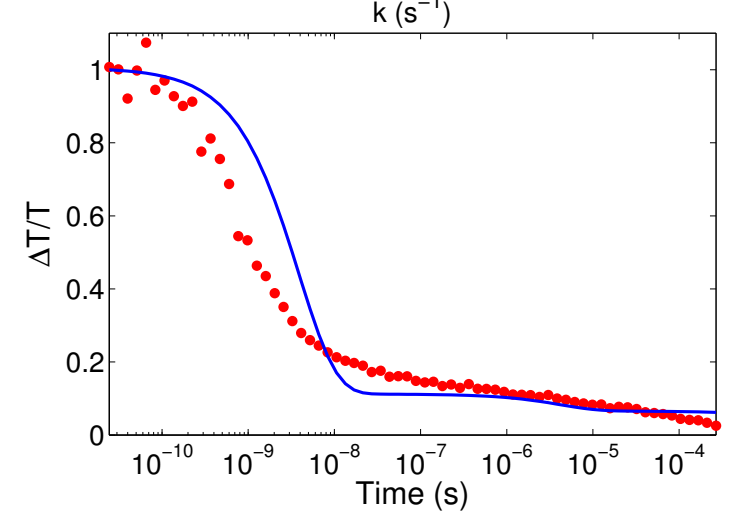

(p) 


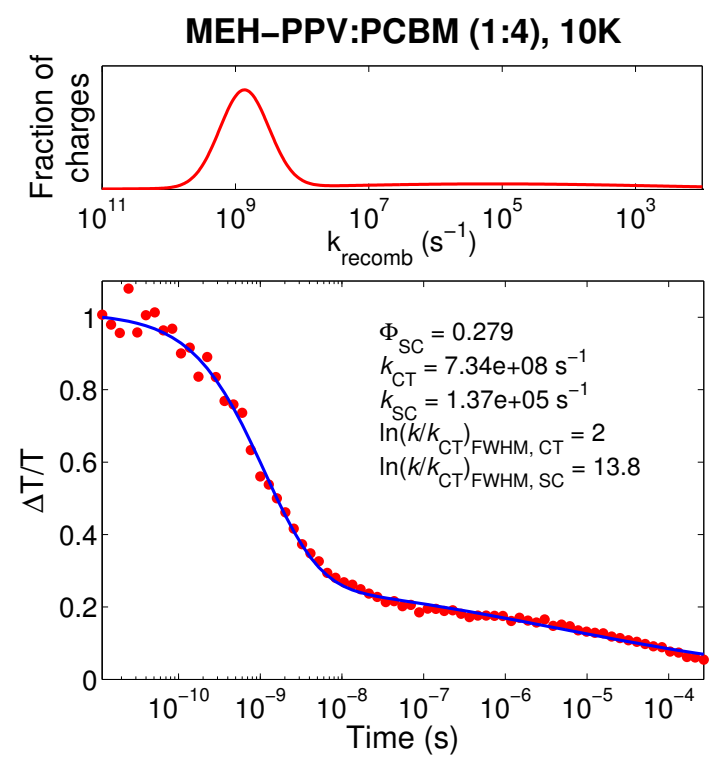

(q)
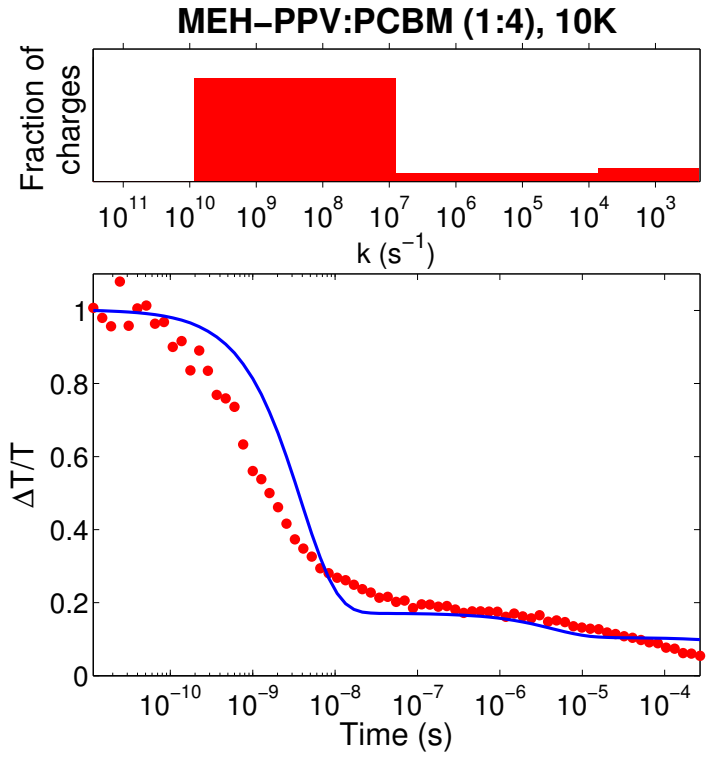

$(\mathrm{r})$

Figure 5.5: Distributed rate constants (either bimodal gaussian or 25 individual rates, logarithmically spaced) used to fit recombination decay in a range of OPV blends.

\subsection{Appendix III: Polarization anisotropy}

Polarization anisotropy decay of the transient absorption signal was measured by inserting a polarizing beamsplitter into the path of the probe pulse after interaction with the sample, splitting the light into components parallel $(\|)$ and perpendicular $(\perp)$ to the excitation pulse. By simultaneously measuring the transient absorption of these components on separate channels, the time- and wavelength-dependent polarization anisotropy $\left.r_{(}(T / T)\right)$ was given by:

$$
r_{\left(\frac{\Delta T}{T}\right)}=\frac{\left(\frac{\Delta T}{T}\right)_{\|}-\left(\frac{\Delta T}{T}\right)_{\perp}}{\left(\frac{\Delta T}{T}\right)_{\|}+2\left(\frac{\Delta T}{T}\right)_{\perp} .}
$$

A non-zero polarization anisotropy indicates some net alignment of the absorption dipoles of the excited states with respect to the excitation pulse (with a maximum possible value of 0.4 for a population of perfectly aligned dipoles with respect to the excitation pulse polarization).

Figure 5.6 shows anisotropy probed at $800 \mathrm{~nm}$, after excitation at $532 \mathrm{~nm}$. For time delay greater than $2 \mathrm{~ns}$, the excitation light was generated as described in 
Section 5.2. For times less than $2 \mathrm{~ns}, 100 \mathrm{fs}$ excitation pulses were generated in an optical parametric amplifier (TOPAS) with pump-probe delay varied using a mechanically controlled delay stage.

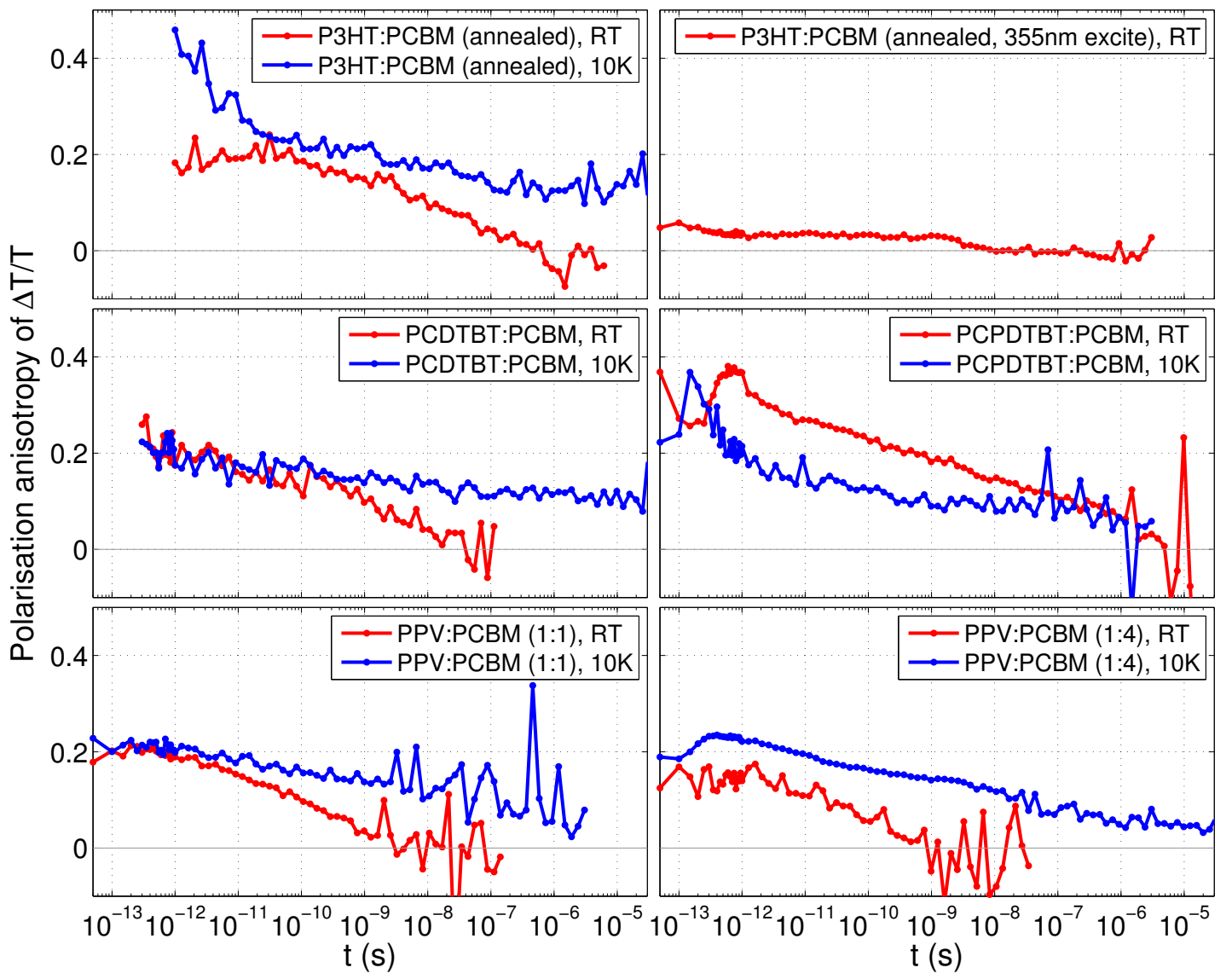

Figure 5.6: Polarization anisotropy decay at $290 \mathrm{~K}$ and $10 \mathrm{~K}$ for the blends studied

Nearly all materials show drastically reduced anisotropy decay at $10 \mathrm{~K}$, due to charges becoming immobile at sufficiently low temperature. The only exception is PCPDTBT:PCBM, which may reflect the fact that we probed the ground-state bleach (GSB) in that case, which contains contributions from all excitations.

Interestingly, in P3HT:PCBM anisotropy is almost completely absent when the sample is excited at $355 \mathrm{~nm}$, a wavelength at which excited states are preferentially generated on the PCBM molecule. Since the $800 \mathrm{~nm}$ probe is only sensitive to cationic absorption within the polymer, we can conclude that no memory of dipole orientation is retained in photogenerated holes from the initial excitation in PCBM. 


\section{Chapter 6}

\section{Ultrafast time-resolved measurement of absorption cross-section and population density via probe-saturation effects in TA spectroscopy}

Abstract

Photoinduced absorption signals measured by transient absorption spectroscopy are typically proportional to the product of absorption cross-section and excited state density. We show that this approximation does not hold at high probe pulse intensities, and introduce the use of probe intensity-dependent spectroscopy to measure the singlet excited-state $\left(\mathrm{S}_{1} \rightarrow \mathrm{S}_{2}\right)$ absorption cross section of the conjugated polymer F8BT as $1.6 \times 10^{-16} \mathrm{~cm}^{2} \pm 40 \%$ at $800 \mathrm{~nm}$ and $3.7 \times 10^{-16} \mathrm{~cm}^{2} \pm 30 \%$ at $900 \mathrm{~nm}$, with no variation over the time window surveyed.

The technique may be useful for quantifying salient parameters in many systems, such as branching yields in systems exhibiting singlet fission or triplet production, or cross-sections required for photophysical models. 


\subsection{Introduction}

Transient absorption spectroscopy has proven to be an extremely successful tool for investigating the nature of excited states in conjugated polymers and chargegenerating blends. However, while it allows accurate determination of time scales associated with excited state processes, the amplitude of the measured signal is almost universally interpreted in a qualitative fashion, with scarce consideration of the absolute value of absorption.

In the case of a photoinduced absorption (PIA), under typical measurement conditions the amplitude of the $\Delta T / T$ signal is proportional to the product of the density $N$ and absorption cross section $\sigma$ of the excited states. Decoupling these two parameters would unlock a wealth of new measurement opportunities. Potential applications include the quantification of branching yields, such as populations of nonemissive triplet excitons in polymer OLEDs or the identification of fission or upconversion processes. The ability to measure the cross section of various excitations (singlet/triplet excitons, bound or free charge polarons) and their dependence on device nanomorphology would enhance our understanding of key phenomena and allow us to fix parameters used in globally fitting photophysical kinetics. Time resolved measurements of excited state absorption cross sections may even illuminate the role played by ultrafast delocalization in free charge photogeneration.

A recent study by Reid and Rumbles [184] similarly seeks to quantify previously qualitative tools. They describe the use of microwave conductivity measurements in conjunction with fluorescence quenching by exciton-charge annihilation to determine the population density - and by extension the absorption coefficient (in their case presented as the GHZ mobility) — of photogenerated polarons in conjugated polymers. They also present a concise review of existing methods, including pulse radiolysis TRMC [185] and chemical doping measurements. [186]

Determining $N$ and $\sigma$ via optical probes is typically done by estimating $N$ based on the number of absorbed pump pulse photons, although this can only be applied to single species systems where no branching occurs, and assumes that $\sigma$ remains constant over time. In appropriate systems, chemical doping (for example, to allow formation of triplets via intersystem crossing from heavy metal atoms [42]) allows quantification of formation rates and therefore absorption cross section. 
In this work, we use the hithero unexplored dependence of transient absorption signal amplitude on probe intensity to decouple $N$ and $\sigma$ for the lowest lying singlet excited state $S_{1}$ of a conjugated polymer film. We find that, at high probe intensity, a sufficient fraction of the exciton population is driven out of $S_{1}$ that the absorption of the probe approaches saturation, reducing the measured $\Delta T / T$ signal. By numerically solving the rate equations describing this process and recreating the probe intensity dependence, we are able to determine time-resolved and wavelength-dependent values of $N$ and $\sigma$. In addition, we find that increasing the energy of the probe photons introduces additional transitions, such as by two-photon excitation of $S_{1}$, for which we are also able to assign absorption cross sections.

\subsection{Qualitative interpretation}

In absorption spectroscopy, one can consider the absorption of light by a volume of sample to take place by a number of absorbing units with population per unit volume $N$, each having an effective (wavelength-dependent) cross-section $\sigma$. In traditional 'weak-probe' absorption spectroscopy, absorption of the probe light by the sample is generally approximated as causing a negligible reduction in ground state population density. It is this approximation that allows formulation of the well-known Beer-Lambert law, $A=N \sigma l$, where $N$ and $\sigma$ are the population density and absorption cross-section of the absorbing species, and $l$ is the absorption length. ${ }^{\mathrm{i}} A$ is the optical density of the sample, defined as $A=-\log \left(I / I_{0}\right)$, the relative attenuation of light intensity $I$ from an initial value $I_{0}$.

It is obvious that under these conditions the absorption (and by extension any measured parameter) will always be proportional to the product $N \sigma$. In situations where we cannot fix either of these values, we can only measure the product $N \sigma$.

It is possible, however, to conceive of a situation where the probing light is sufficiently intense as to drive a significant population of the absorbing species to a different state, potentially changing the optical density experienced by any subsequent probing light (as illustrated in Figure 6.1). This saturation would occur more readily for a sample with low number of excitations and large absorption cross section than for a one with high number of exciations and low cross section,

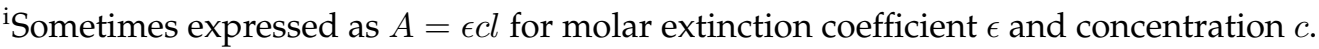


even if these two hypothetical materials had an identical product $N \sigma$. With some knowledge of the excited state landscape for the material, a set of differential equations could be formulated describing the rates of transitions, and solved numerically to recreate the dependence of probe absorption on probe intensity.
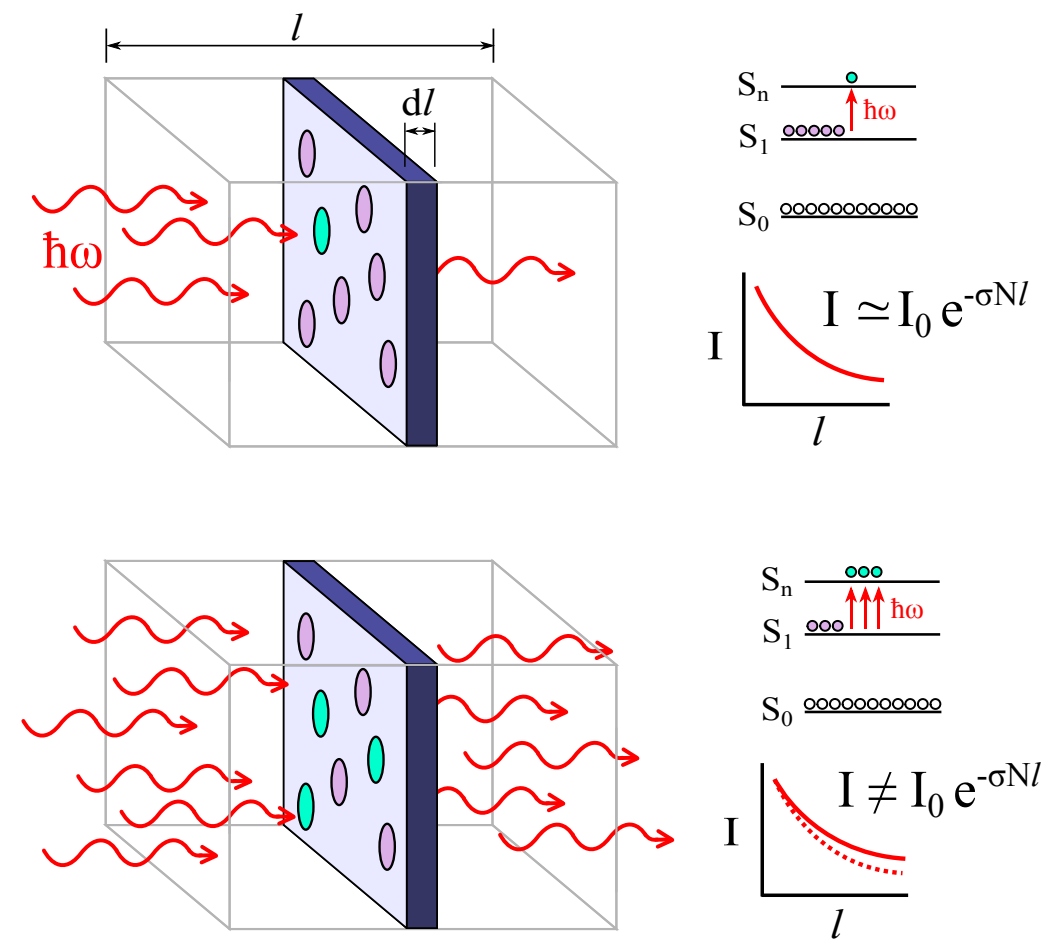

Figure 6.1: The Beer-Lambert approximation fails under sufficient probe intensity.

\subsubsection{Standard TA spectroscopy of F8BT}

To investigate whether we could access a regime where probe-saturation effects could be observed, we decided to perform probe intensity-dependent TA of the semiconducting polymer poly[(9,9-di-n-octylfluorenyl-2,7-diyl)alt-(benzo[2,1,3]thiadiazol-4,8- diyl)] (F8BT). This material was selected for its relatively well

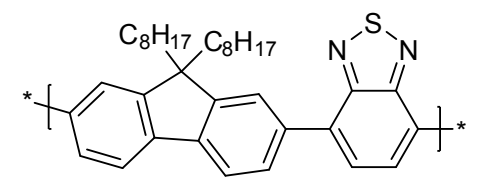

Figure 6.2: F8BT structure understood excited state behavior and low rate of inherent charge generation. In this sense, it provides a useful benchmark with which to test our probe-saturation model. 


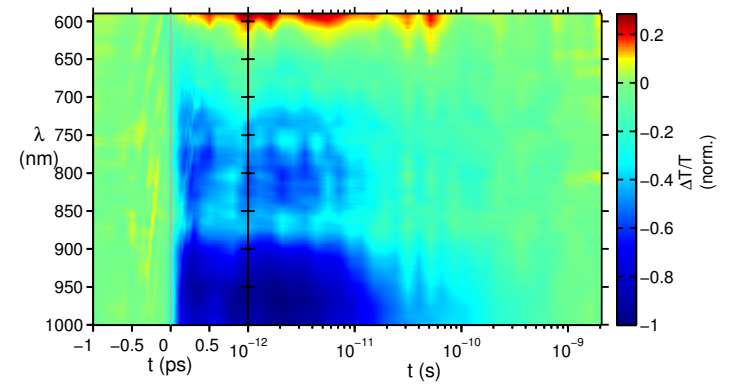

(a)

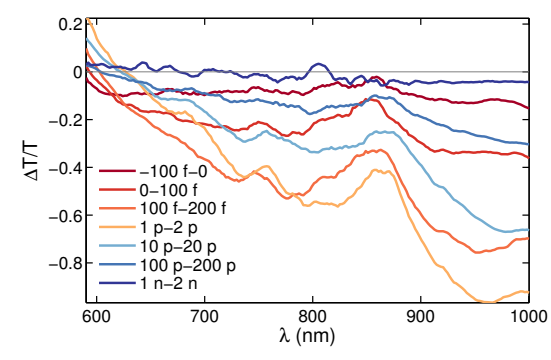

(b)

Figure 6.3: Broadband transient absorption map and spectra of a F8BT film excited at $400 \mathrm{~nm}$

The ideal film for observing saturation of the probe absorption would be optically dense, to maximize the absolute amplitude of the $\Delta T / T$ signal and, by extension, any deviations due to probe saturation effects. Additionally, the film should have a very uniform thickness, since (as we will see below) film thickness is a key parameter to fix when applying any model describing probe absorption. A solution of F8BT ${ }^{\mathrm{ii}}$ in choloroform $\left(35 \mathrm{mg} \mathrm{m}^{-1}\right)$ was spin coated onto fused silica substrates at 2000 RPM. The resulting film thickness was measured by Dektak profilometer to be $705 \mathrm{~nm}$, and had an optical density of 1.82 at $400 \mathrm{~nm}$.

To check for interchain processes from aggregated polymer chains, a second film was cast where the semiconducting polymer was dispersed in an inert matrix of polystyrene. The F8BT:PS film was prepared by dissolving a 1:10 ratio of F8BT and polystyrene in chloroform with a total concentration of $63 \mathrm{mg} \mathrm{ml}^{-1}$. Spin coating at 2000 RPM yielded films with a uniform thickness of $1440 \mathrm{~nm}$ and optical density of 0.44 at $400 \mathrm{~nm}$.

TA spectroscopy was first undertaken with a broadband probe (supercontinuum generated by pumping a $3 \mathrm{~mm}$ sapphire plate with $100 \mathrm{fs}$ pulses at $1300 \mathrm{~nm}$ generated in an optical parametric amplifier), allowing us to investigate the excited state landscape from $600-1000 \mathrm{~nm}$. Figure 6.3a shows the resulting TA map of the neat F8BT film after $100 \mathrm{fs}$ excitation at $400 \mathrm{~nm}$. The spectral region shown is dominated by an excitonic photoinduced absorption extending into the near-infrared. The edge of a positive $\Delta T / T$ signal is apparent near $600 \mathrm{~nm}$, and is likely due to stimulated emission. Some structure in the spectra between $800-900 \mathrm{~nm}$ may be

\footnotetext{
ii American Dye Source, Inc., ADS133YE, MW 15.000-200.000
} 
due to residual $800 \mathrm{~nm}$ light in our probe pulse having different temporal and spatial characteristics than the white light continuum. The entire spectrum decays uniformly, allowing us to assign all features to the excitonic first singlet excited state $\left(\mathrm{S}_{1}\right)$.

Since they are due to a single population of excited states (i.e. $N_{1}$ is fixed), the spectra shown in $6.3 \mathrm{~b}$ give us an intuitive feeling for the values that should be returned by our probe saturation model. For example, we expect to find a higher excited-state absorption cross-section at $900 \mathrm{~nm}$ than at $800 \mathrm{~nm}$, but equal $N_{1}$ at both wavelengths, decaying in time as per the kinetics shown in Figure 6.3a.

Accurate fitting of absorption cross-section requires that we know the spatial distribution of pump and probe energy density. We measured the shape of each of these beams by placing the exposed CCD from a common webcam in the sample plane of our experiment, as described in Chapter 2. Using the published pixel size and pitch of the CCD, gaussian fits of the data gave us the FWHM of each beam.

\subsubsection{Probe intensity-dependence}

Before we can use probe-saturation effects to probe any physical parameters, we must first check to see whether we are in fact able to observe any dependence of signal magnitude on probe intensity! The effect is likely to be small, so we design the experiment with the aim of maximizing the stability and repeatability of the collected $\Delta T / T$ values at a given configuration of pump-probe delay, intensity, and wavelength.

In order to quantitatively apply any model, we will need to know the number of photons in our probe pulse. We therefore use a single wavelength probe, starting with the $800 \mathrm{~nm}$ fundamental output of our chirped-pulse amplifier, which exhibits excellent shot-to-shot stability. The probe light was passed through a 50/50 beam splitter prior to interaction with the sample, with one of the subsequent pulses used as a reference probe as described in Section 2.

Probe pulse intensity was adjusted with a variable-attenuator prior to interaction with the sample, and a second attenuator after the sample, to maintain an ideal intensity at the spectrometer with which to take full advantage of the dynamic range of our photodiode array and digitization circuitry. Probe intensity at the sample ranged from $47 \mu \mathrm{J} \mathrm{cm}^{-2}$ pulse $^{-1}$ (limited by the sensitivity of our photodiode array) to $990 \mu \mathrm{J} \mathrm{cm}^{-2}$ pulse $^{-1}$ (just below the threshold for significant 
two-photon excitation of the sample by the probe, determined by visual detection of fluorescence).

Since we are not initially interested in kinetics, we can collect relatively large datasets at each probe-light intensity and time point of interest. For the measurements presented here, the probe light was dispersed over $\sim 20$ pixels of our photodiode array, and 30 sets of 1,000 $\Delta T / T$ values were collected at each probe intensity. The error bars represent one standard deviation for that set of $20 \times 30$ $\Delta T / T$ values.

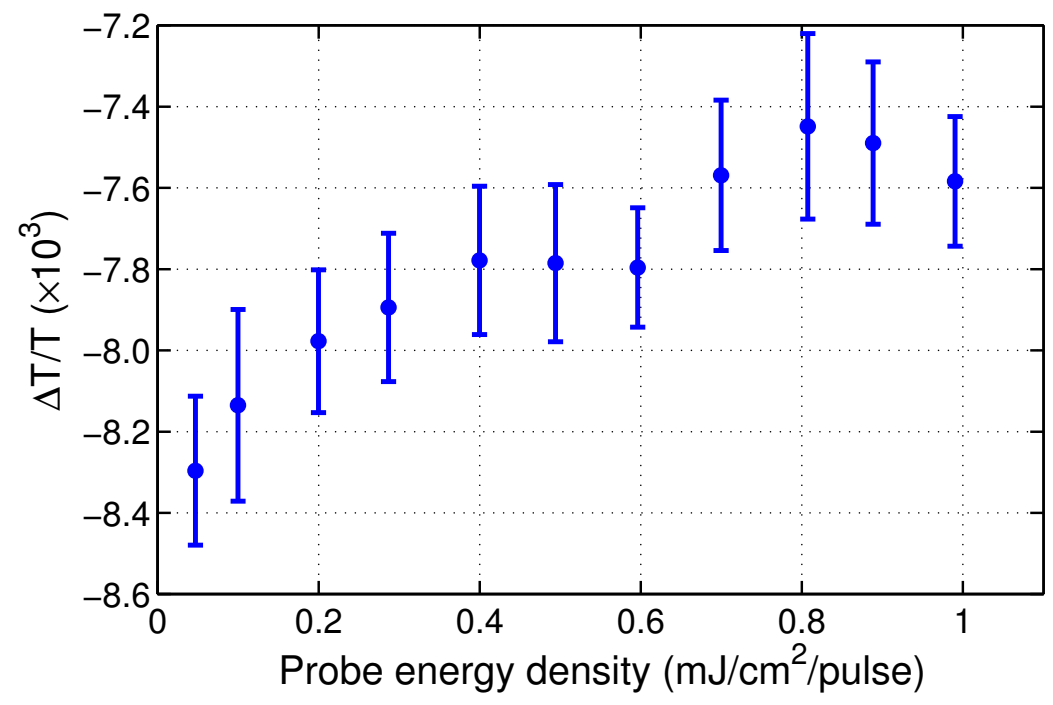

Figure 6.4: TA spectroscopy of the PIA at $800 \mathrm{~nm}$ in neat F8BT with pump-probe delay fixed at $10 \mathrm{ps}$ and $400 \mathrm{~nm}$ excitation fixed at $20 \mu \mathrm{J} \mathrm{cm}^{-2}$ pulse $\mathrm{s}^{-1}$. We see a reduction in the excited state absorption as the probe intensity is increased, approaching saturation of the absorbing population. Error bars represent one standard deviation for the collected values at each probe intensity.

Excitation pulses with an energy density of $20 \mu \mathrm{J} \mathrm{cm}^{-2}$ pulse ${ }^{-1}$ at $400 \mathrm{~nm}$ were obtained by frequency doubling our $800 \mathrm{~nm}$ fundamental in a type-I BBO crystal, exhibiting superior shot-to-shot and long term stability than pulses generated in a parametric amplifier. By keeping the size of the pump spot at the sample very large compared to the probe, we both simplify the interactions (as we can approximate the probe beam as passing through a uniformly excited region of the sample), and minimize the sensitivity of $\Delta T / T$ amplitude to pump and probe alignment. The spot sizes were measured before every experimental run, and were 
typically found to have full width at half maximum (FWHM) values of $1120 \mu \mathrm{m}$ and $150 \mu \mathrm{m}$ in the sample plane for the pump and probe, respectively.

Our sample was kept under dynamically pumped vacuum during the experiment, to minimize photodegradation. While the probe intensities employed here will strike many ultrafast spectroscopists as being sufficient to obliterate a thin film in short order, we are protected by virtue of our probe wavelength being far from resonance with the ground state transition. In this case, the density of excitations in the sample is effectively limited by the pump, which is fixed at a modest $20 \mu \mathrm{J} \mathrm{cm}^{-2}$ pulse $^{-1}$.

However, as data across a full range of probe intensities may take several hours to collect, some photodegredation may take place. Additionally, we experience drift of the TA signal amplitude due to fluctuations in beam intensity and alignment. Such drift is not a critical issue for typical qualitative TA experiments, but is severly problematic here, as we need to compare absolute signal magnitude across several meaurements. In order to remove such spurious influences, we employed a measurement sequence whereby a low-intensity probe measurement was collected after each high-intensity value. In the case shown in Figure 6.4, the sequence was $[47,99,47,200,47,287, \ldots 990,47] \mu \mathrm{J} \mathrm{cm}{ }^{-2}$ pulse $^{-1}$. The initial low probe intensity measurement was then used as a reference point, with any deviation of the following low power results used to determine a scaling factor by which to correct their associated high intensity measurement. These corrections tended to be fairly small, but did improve the quality of the fits introduced in the following section.

The result of our preliminary probe-saturation experiment is presented in Figure 6.4. As predicted, we see a decrease in the amplitude of the photoinduced absorption with increasing probe intensity, from $-8.30(18) \times 10^{-3}$ at $47 \mu \mathrm{J} \mathrm{cm}^{-2}$ pulse $^{-1}$ to $-7.58(16) \times 10^{-3}$ at $990 \mu \mathrm{J} \mathrm{cm}^{-2}$ pulse $^{-1}$, where the stated errors are the standard deviation of the collected values for each data point.

\subsection{A numerical model of TA probe absorption}

\subsubsection{Relevant transitions and rates}

In order to develop a model that describes the absorption of our probe at intensities beyond the Beer-Lambert regime, we must consider the various optical transitions 
taking place in our sample, and the effect of those transitions on the populations of the relevant states.

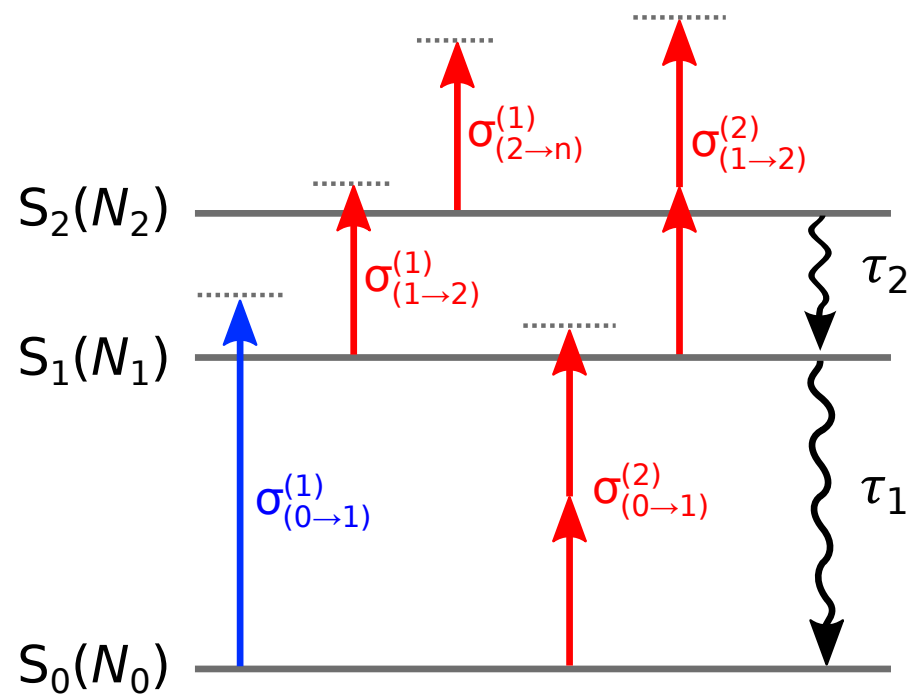

Figure 6.5: Schematic for a simple semiconducting polymer such as F8BT, showing the relevant transitions that determine the population density $N_{1}$ of the lowest singlet excited state $\mathrm{S}_{1}$. Associated cross-sections for single-photon $\left(\sigma^{(1)}\right)$ and two-photon $\left(\sigma^{(2)}\right)$ absorption are shown, along with excited-state lifetimes $\tau_{1}$ and $\tau_{2}$.

Let us consider the first three singlet excited states of our system $S_{0}, S_{0}$, and $S_{2}$, with accompanying population densities $N_{0}, N_{1}$, and $N_{2}$ as shown in Figure 6.5. Electrons in each state can undergo either single- or two-photon absorption, with effective absorption cross-sections for these events given by $\sigma^{(1)}$ and $\sigma^{(2)}$. Higher order processes are also possible, but occur with negligible rate at the photon fluences relevant to our experiment. Relaxation can occur either radiatively or via internal conversion, with lifetimes $\tau_{1}$ and $\tau_{2}$. In the case of F8BT, relaxation from $\mathrm{S}_{1}$ has been shown to be $\sim 50 \%$ radiative [187]).

In a typical TA experiment, the pump beam drives the ground state absorption (with absorption cross-section $\sigma_{0 \rightarrow 1}^{(1)}$ ) to establish an excited state population $\mathrm{N}_{1}$. The goal of this work is to develop a time-dependent quantitative measurement of $N_{1}$ and $\sigma_{1 \rightarrow 2}^{(1)}$. The effect of the other transitions must be considered, either by quantifying their contribution, or by arranging the experiment such that their contributions are negligible.

Our numerical model will be based on the following set of rate equations, 
developed from those formulated by Zhang et al. [188]:

$$
\begin{aligned}
\frac{d N_{0}}{d t} & =\frac{N_{1}}{\tau_{1}}-N_{0} P_{0 \rightarrow 1}(r, z, t), \\
\frac{d N_{1}}{d t} & =N_{0} P_{0 \rightarrow 1}(r, z, t)-N_{1} P_{1 \rightarrow 2}(r, z, t)-\frac{N_{1}}{\tau_{1}}+\frac{N_{2}}{\tau_{2}}, \\
\frac{d N_{2}}{d t} & =N_{1} P_{1 \rightarrow 2}(r, z, t)-N_{2} P_{2 \rightarrow n}(r, z, t)-\frac{N_{2}}{\tau_{2}},
\end{aligned}
$$

with the transition rates given by

$$
\begin{aligned}
& P_{0 \rightarrow 1}(r, z, t)=\frac{\sigma_{0 \rightarrow 1}^{(1)}(\omega) I(r, z, t)}{\hbar \omega}+\frac{\sigma_{0 \rightarrow 1}^{(2)}(\omega) I^{2}(r, z, t)}{2 \hbar \omega}, \\
& P_{1 \rightarrow 2}(r, z, t)=\frac{\sigma_{1 \rightarrow 2}^{(1)}(\omega) I(r, z, t)}{\hbar \omega}+\frac{\sigma_{1 \rightarrow 2}^{(2)}(\omega) I^{2}(r, z, t)}{2 \hbar \omega}, \\
& P_{2 \rightarrow n}(r, z, t)=\frac{\sigma_{2 \rightarrow n}^{(1)}(\omega) I(r, z, t)}{\hbar \omega},
\end{aligned}
$$

where $\omega$ and $I(r, z, t)$ are light frequency and intensity. The temporal and spatial disribution of the pump and probe pulses can be described by Gaussian distributions. Additionally, since we can assume the pulses propagate collinearly through the sample, the geometry becomes cylindrically symmetric. The spatial coordinates $(r, z)$ therefore represent radial distance from, and position along, the propagation axis respectively. We define $\left(r_{0}, z_{0}\right)$ as the point at the center of the pump and probe beams, on the incident face of the sample.

$P_{0 \rightarrow 1}, P_{1 \rightarrow 2}, P_{2 \rightarrow n}$ are transition rates, which correspond directly to the absorption that we measure in our TA experiment. While the rate equations cannot be analytically solved, they do enable us to numerically predict the spatial and temporal dependence of absorption on $N$ and $\sigma$.

\subsubsection{Implementation of the numerical model}

For the following analysis we assume that the pump-probe delay is greater than $\sim 200 \mathrm{fs}$, so that absorption of the pump and probe can be treated as two independent events. Additionally, the time between sequential shot pairs (333 $\mu$ s for the $3 \mathrm{kHz}$ repetition rate of our laser) is much greater than either of the excited state lifetimes (Figure 6.3a shows nearly complete relaxation after $1 \mathrm{~ns}$ ). We can therefore consider each pump-probe pulse pair to be independent events, acting on initial excited state population densities $\left(N_{1}=N_{2}=0\right)$. 
We first consider pump absorption, which determines the population density $N_{1}(r, z)$ experienced by the probe pulse. Now since $N_{1}$ is to be one of our fitted parameters, we do not want to calculate it outright from measurements of pump intensity. However, we do need to take into condideration the variation in $N_{1}$ with radial distance $r$ and depth into the sample $z$. As long as our excitation density is below the threshold for non-linear interactions such as exciton-exciton annihilation, we can assume that all excited states decay uniformly, maintaining shape of this spatial distribution throughout the sample regardless of the absolute population density or the value of the pump-probe delay and of the decay rates $\tau_{1}$ and $\tau_{2}$. If we define this dimensionless spatial distribution as $N_{1}^{\prime}(r, z)$, our fitted paramater will therefore be $N_{1, \text { peak, }}$ where $N_{1}(r, z)=N_{1, \text { peak }} N_{1}^{\prime}(r, z)$.

Absorption of the pump pulse is well described by the Beer-Lambert equation, due to the relatively low pulse intensity (compared to the probe pulse) and high density of ground-state absorbers. Measurements of the pump beam width $\left(r_{\mathrm{FWHM}}=1120 \mu \mathrm{m}\right)$ allow us to determine photon flux per unit area at the incident face of the sample as a function of radial distance $r$. Since we also know film thickness $l$ and optical density $A$, the pump intensity throughout the sample (for a spatially and temporally Gaussian pulse) is given by

$$
I(r, z, t)=I_{\text {peak }} \exp \left(-4 \ln 2\left[\left(r / r_{\mathrm{FWHM}}\right)^{2}+\left(t / t_{\mathrm{FWHM}}\right)^{2}\right]-z A / l\right) .
$$

Integrating with time over a single pump pulse yields the energy density per pulse, we get

$$
U(r, z)=U_{\text {peak }} \exp \left(-4 \ln 2\left(r / r_{\text {FWHM }}\right)^{2}-z A / l\right) .
$$

Since every absorbed photon generates a single excited state, the population density in $S_{1}$ is given by

$$
N_{1}(r, z)=\frac{\mathrm{d}}{\mathrm{d} z}\left(\frac{-U(r, z)}{\hbar \omega}\right),
$$

which (unsurprisingly) exhibits the same spatial distribution as pump intensity, and can be expressed as:

$$
N_{1}(r, z)= \begin{cases}0, & z<0, \\ N_{1, \text { peak }} \exp \left(-4 \ln 2\left(r / r_{\text {FWHM }}\right)^{2}-z A / l\right), & 0<z<l, \\ 0, & z>l .\end{cases}
$$


We can now consider absorption of the probe pulse, which is a little trickier as we cannot assume exponential (Beer-Lambert) absorption. Instead, we must include time as a variable in order to account for changes in the population densities $N_{0}, N_{1}$ and $N_{2}$ that take place as the pulse propagates through the sample.

Our approach is to split the system into $m$ steps of $r\left(0<r<2 r_{\mathrm{FWHM}}\right)$ and $n$ steps of $z\left(-6 c t_{\mathrm{FWHM}}<z<l\right)$. Our propagation axis is defined in order to accomodate both the full probe pulse (over a range $6 t_{\mathrm{FWHM}}$, outside of which intensity is negligible) and our sample thickness $l$.

We define a $m \times n$ array representing population density $N_{1}$, which is initialized according to equation 6.6. We define a second $m \times n$ array representing probe intensity $I$. The temporal dependence of our pulse can be described by a gaussian with $t_{\mathrm{FWHM}}=100 \mathrm{fs}$, which is converted into distance by the speed of light $c$ and used to initialize the probe intensity array with the probe pulse centered at $z=-3 c t_{\mathrm{FWHM}}$ as

$$
I\left(r, z, t_{0}\right)=I_{\text {peak }} \exp \left(-4 \ln 2\left(\left(\frac{r}{r_{\text {FWHM }}}\right)^{2}+\left(\frac{z+3 c t_{\text {FWHM }}}{c t_{\mathrm{FWHM}}}\right)^{2}\right)\right) .
$$

The time-dependent interaction between the probe and sample is then determined by increasing the $z$ position of the intensities in $I$ one step at a time $(I(r, z+\delta z)=I(r, z))$, and recalculating both the $N_{1}$ and $I$ array at each iteration using the rate equations given in 6.1. Since the total time for the probe to propagate through the sample is several orders of magnitude shorter than the lifetimes $\tau_{1}$ and $\tau_{2}$, we can ignore excited state relaxation for this calculation.

As our probe light is far from resonance with the single photon ground state absorption, we can clearly take $\sigma_{0 \rightarrow 1}^{(1)}=0$. For now, we will also take $\sigma_{0 \rightarrow 1}^{(2)}=$ $\sigma_{2 \rightarrow n}^{(1)}=0$, but will return to them in the following section.

For each time iteration, we find the change in probe intensity and population density due to the absorption processes described by the transition rates in equation 6.3.1:

$$
\begin{aligned}
I(r, z+\delta z, t+\delta t)=I(r, z, t) & -I(r, z, t) N_{1}(r, z, t) \sigma_{1 \rightarrow 2}^{(1)} \delta z \\
& -I^{2}(r, z, t) N_{1}(r, z, t) \sigma_{1 \rightarrow 2}^{(2)} \delta z,
\end{aligned}
$$




$$
\begin{array}{r}
N_{1}(r, z, t+\delta t)=N_{1}(r, z, t)- \\
-\frac{I(r, z, t) N_{1}(r, z, t) \sigma_{1 \rightarrow 2}^{(1)}}{\hbar \omega} \\
-\frac{I^{2}(r, z, t) N_{1}(r, z, t) \sigma_{1 \rightarrow 2}^{(2)}}{2 \hbar \omega}, \\
N_{2}(r, z, t+\delta t)=N_{2}(r, z, t)+\frac{I(r, z, t) N_{1}(r, z, t) \sigma_{1 \rightarrow 2}^{(1)}}{\hbar \omega} \\
+\frac{I^{2}(r, z, t) N_{1}(r, z, t) \sigma_{1 \rightarrow 2}^{(2)}}{2 \hbar \omega} .
\end{array}
$$

The differing factor of two in the two-photon absorption term, is a result of the number of photons + excitations not being conserved in this process. Also note the new intensity has an offset along the $z$ axis to account for propagation of the pulse through the sample.

Once enough iterations have taken place for the pulse to pass completely through the sample, we convert the $I$ array from cylindrical to cartesian coordinates in order to reform the imaged probe spot in the plane of the sample. The measured probe intensity $T$ is then found by integrating $I$ over $x, y$, and $z$. By taking the normalized difference of this value with the the probe intensity $T$ prior to interaction with the sample, we obtain our calculated value of $\Delta T / T$ that can be compared with those obtained experimentally.

It is worth noting here that, when calculated in SI units, the above calculation for a typical system involves multplying numbers of vastly different magnitudes $\left(\hbar \sim 10^{-34}, \sigma \sim 10^{-16} \mathrm{~cm}^{2}=10^{-20} \mathrm{~m}^{2}, N \sim 10^{17} \mathrm{~cm}^{-3}=10^{23} \mathrm{~m}^{-3}\right)$. Our model therefore works in base units of nanometres, which serves to keep variables much closer to unity, and avoid numerical errors such as stack overflows.

Figure 6.6 shows a set of probe intensity-dependent $\Delta T / T$ curves for a range of cross-sections and initial population densities. In this case, $\sigma_{1 \rightarrow 2}^{(2)}=0$, and the product $\sigma_{1 \rightarrow 2}^{(1)} N_{1, \text { peak }}$ was fixed to 10 . This corresponds to a set of systems that would generate identical signals in a traditional TA experiement. We see that, as expected from our intial qualitative explaination discussed in Section 6.2, the set of absorbers with high $N$ and low $\sigma$ are saturated more easily by the probe pulse. As we show in the following section, this dependence will enable us to fit our model to a range of experimental data and obtain time-resolved values of $N$ and $\sigma$.

At this stage, the only free values in our model are $N_{1 \text {,peak }}$ and the set of absorption cross-sections for whichever interactions we choose to include. The model 

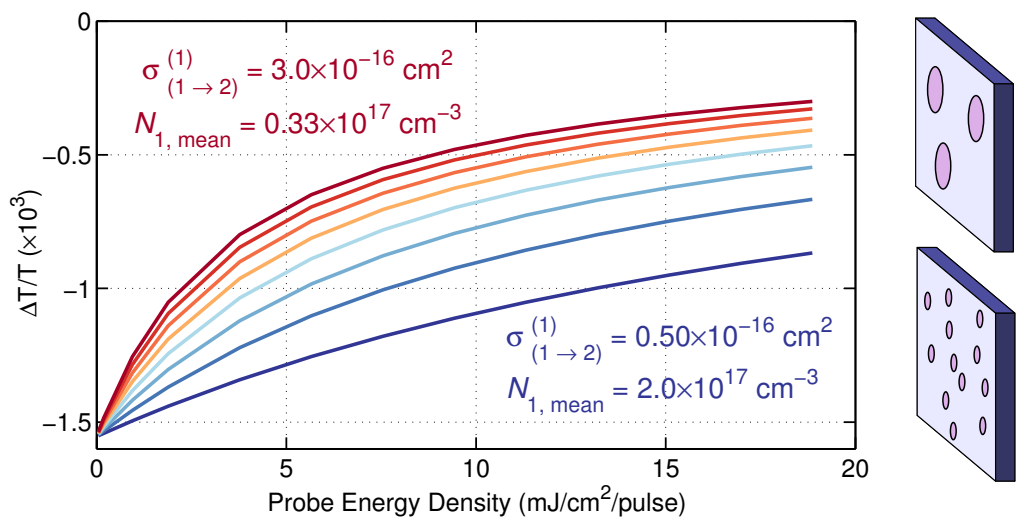

Figure 6.6: Simulated probe intensity dependence of photoinduced absorption for a series of absorbing species where $N$ and $\sigma$ are varied in such a way that the product $N \sigma$ remains constant. It can be seen that the different cases are indistinguishable under low probe intensity, but as probe intensity is increased, the Beer-Lambert law becomes invalid $(A \neq N \sigma l)$, decoupling $N$ and $\sigma$.

is easily expandable, with additional transitions able to be added to equations 6.9 and 6.10 from the rates given in equation 6.3.1 or variations thereof for any system of interest. Each additional transition added to the model adds a single free parameter in the form of the cross-section $\sigma$ for that process. These parameters can be fixed to a value based on prior knowledge of the system, or left as a free parameter to be fitted by a nonlinear minimisation algorithm.

\subsection{Results}

Figure 6.7 shows the data initially presented in Figure 6.4, with our probe saturation model described in Section 6.3.2 fitted using the Nelder-Mead nonlinear minimization algorithm (in MATLAB) to minimize the sum of squares of the residuals between the experimental data and model. The two free parameters were initial $S_{1}$ population density $N_{1}$ and $S_{1}$ single photon absorption cross-section $\sigma_{1 \rightarrow 2}^{(1)}$. The fitted curve falls within one standard deviation of the collected datasets (indicated by the error bars). The lower panel of Fig. 6.7 gives us an indication of the variation of $N_{1}$ throughout the film, and of how saturated the $S_{1 \rightarrow 2}$ transition is being driven by the probe beam at high intensities. At the center of the beam, 
on the incident face of the sample (i.e. $\left(r_{0}, z_{0}\right)$ ), almost half of the population of $S_{1}$ has been excited to $S_{2}$ at the highest probe intensity $\left(990 \mu \mathrm{J} \mathrm{cm}^{-2}\right.$ pulse $\left.^{-1}\right)$.

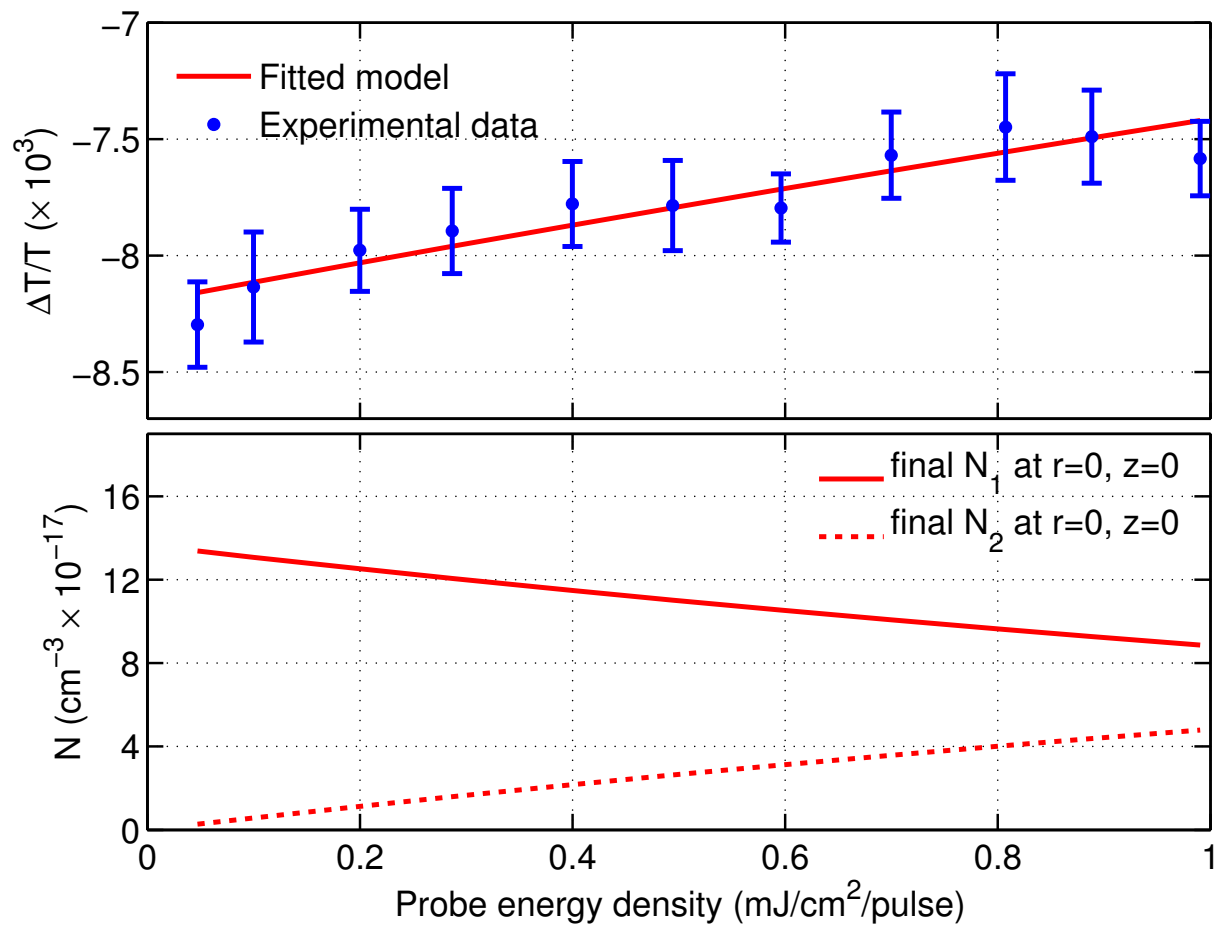

Figure 6.7: Probe intensity dependence of the differential transmission of F8BT, pumped at $400 \mathrm{~nm}\left(20 \mu \mathrm{J} \mathrm{cm}^{-2}\right.$ pulse $\left.^{-1}\right)$ and probing at $800 \mathrm{~nm}$ with a fixed pumpprobe delay of $10 \mathrm{ps}$. Error bars show one standard deviation of the collected values. The red line is a fit from our numerical model, with $\sigma_{1 \rightarrow 2}^{(1)}=1.84 \times 10^{-16}$ $\mathrm{cm}^{2}$ and mean $N_{1}=6.34 \times 10^{17} \mathrm{~cm}^{-3}$. Also shown are calculated population densities of $S_{1}$ and $S_{2}$ at the most saturated point in the sample $(r=0, z=0$ corresponds to the center of the beam, on the incident face of the sample). We see that at the highest probe intensity, almost half of the excitations are driven from $S_{1}$ into $S_{2}$ by the probe.

We can check the validity of our fitted parameters by measuring pump beam power and calculating the expected population density $N_{1}$ from equation 6.6. At the center of the pump beam, this calculation gives a population density that differs by a factor of $\ln 2$ from the commonly used (and somewhat erroneous) 'top-hat' beam profile [189].

Taking our pump beam FWHM as $1120 \mu \mathrm{m}$, for the neat F8BT sample excited with an energy density of $20 \mu \mathrm{J} \mathrm{cm}^{-2}$ pulse $^{-1}$, we estimate a mean initial popu- 
lation density $N_{1}$ of $3.96 \times 10^{17} \mathrm{~cm}^{-3}$ within the excited region sampled by the probe pulse. While on the same order of magnitude as our our fitted value of $6.34 \times 10^{17} \mathrm{~cm}^{-3}$, it suggests that our model is overestimating the population density by a factor of $\sim 3$, taking into account that approximately half of the TA signal has decayed by a time delay of 100 ps.

To investigate the behaviour of our model under a range of input conditions, probe intensity dependent datasets were collected at three pump-probe delays (500 fs, $30 \mathrm{ps}$, and $100 \mathrm{ps})$, at two excitation densities $\left(10 \mu \mathrm{J} \mathrm{cm}{ }^{-2}\right.$ pulse $^{-1}$ and $20 \mu \mathrm{J} \mathrm{cm}^{-2}$ pulse $^{-1}$ ), and at two probe wavelengths ( $800 \mathrm{~nm}$ and $900 \mathrm{~nm}$ ). The range of behaviours expected under these variables is simple to predict. We should see constant $\sigma_{1 \rightarrow 2}^{(1)}$ but decreasing $N_{1}$ with increasing pump-probe delay, in proportion to the kinetics shown in Figure 6.3a. Similarly, $N_{1}$ should scale linearly with pump energy density, but $\sigma_{1 \rightarrow 2}^{(1)}$ should be unaffected. Finally, our $900 \mathrm{~nm}$ probe should measure greater $\sigma_{1 \rightarrow 2}^{(1)}$ than at $800 \mathrm{~nm}$ (see Figure 6.3b), while $N_{1}$ should be independent of probe wavelength.
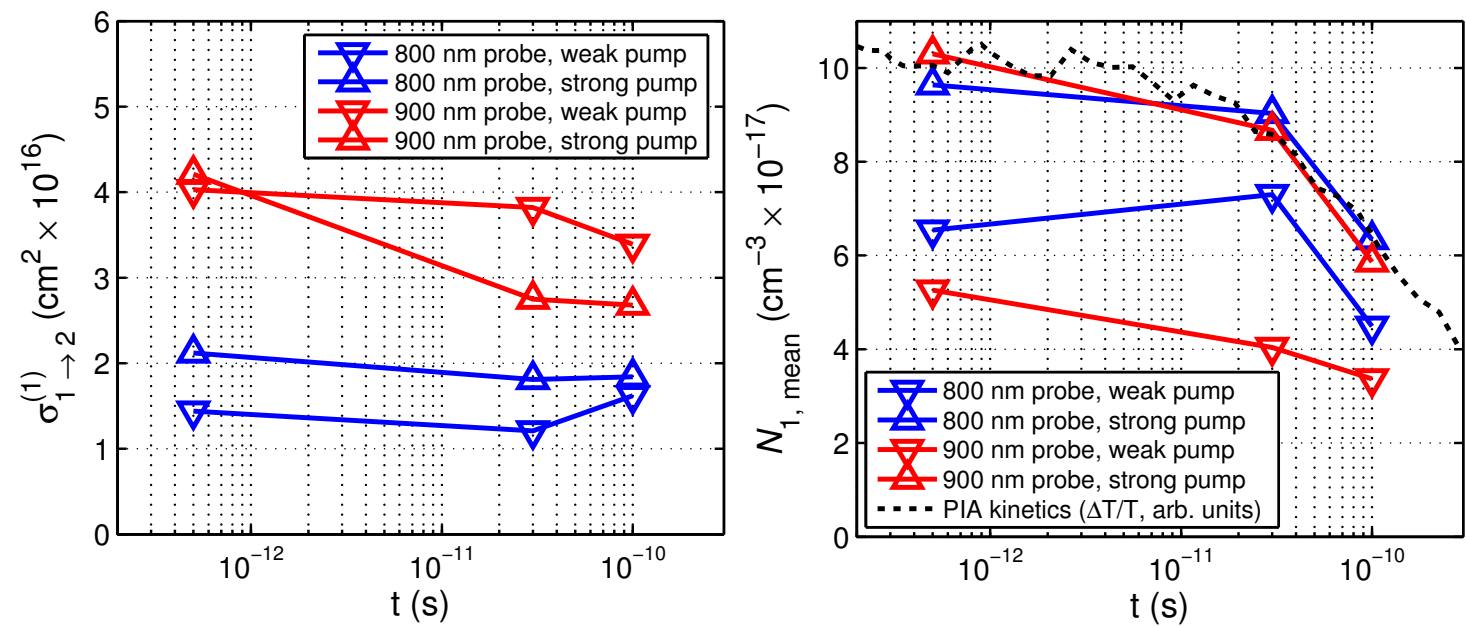

Figure 6.8: Fitted values of $\sigma_{1 \rightarrow 2}^{(1)}$ and $N_{1}$ to probe intensity dependent TA of a neat F8BT film. As expected, $\sigma_{1 \rightarrow 2}^{(1)}$ is dependent on probe wavelength but independent of excitation density. The reverse is true for $N_{1}$, which also decays in proportion to the photoinduced absorption measured from $650-750 \mathrm{~nm}$ by standard TA (see Fig. $6.3 a)$.

Figure 6.8 shows the time, excitation density, and probe wavelength dependence of our fitted parameters $N_{1}$ and $\sigma_{1 \rightarrow 2}^{(1)}$ for the neat F8BT film. While there are a few outlying points, in general the fitted parameters very closely reflect the 
changing experimental configuration. Fitted absorption cross section shows no trend with pump-probe delay time or excitation density, with $\sigma_{1 \rightarrow 2}^{(1)}(900 \mathrm{~nm}) \approx$ $2 \sigma_{1 \rightarrow 2}^{(1)}(800 \mathrm{~nm})$, as expected from the standard TA spectroscopy. The population density is found to decay in excellent agreement with the TA kinetics, which are shown as a grey dashed line with appropriate scaling in order to compare with fitted $N_{1}$.

The range of experimental configurations was repeated with the F8BT:polystyrene film, in order to check for any effects due to interchain interactions or morphology. The results are shown in Figure 6.9. While the blended sample was significantly thicker than the neat film, the lower density of F8BT resulted in a less optically dense film ( $A=0.44$, c.f. 1.82 at $400 \mathrm{~nm}$ for the neat film). The result was a lower absolute $\Delta T / T$ signal magnitude, and therefore somewhat noisier datasets. Satisfyingly, the fitted cross-sections are in good agreement with the blended film, while excited state population density was accordingly lower than in the blend.
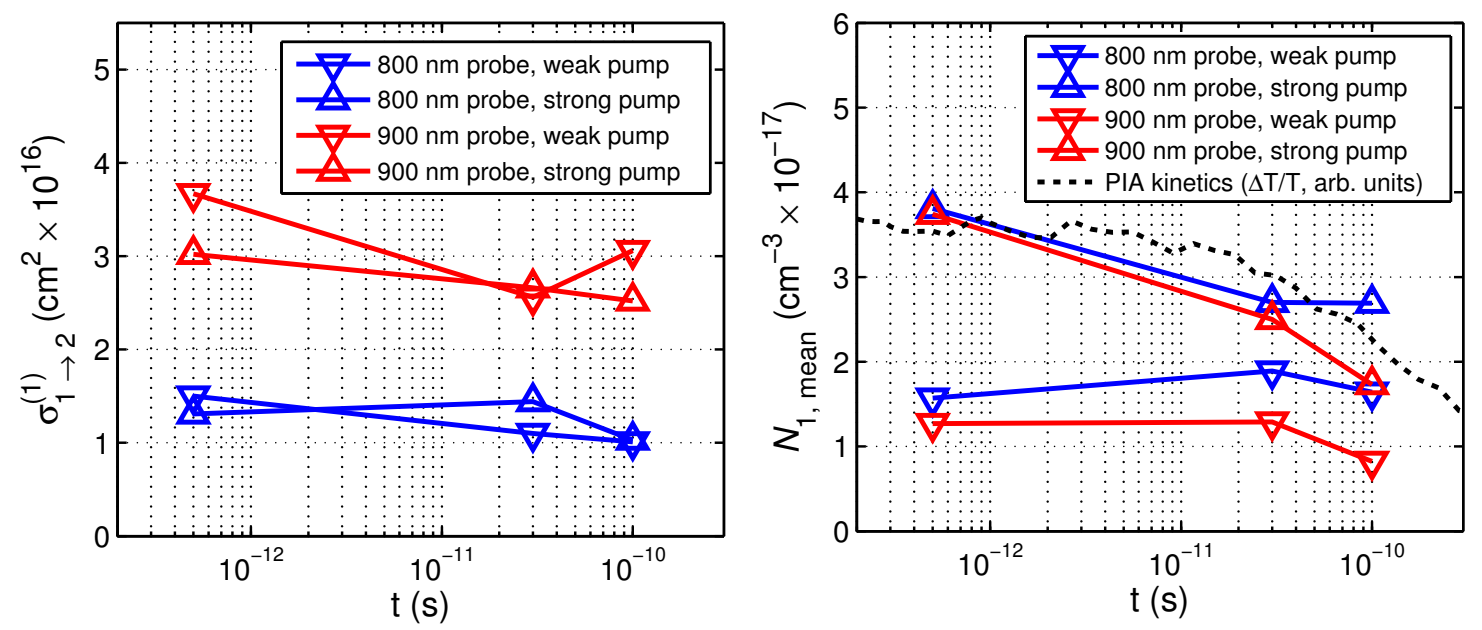

Figure 6.9: Fitted values of $\sigma_{1 \rightarrow 2}^{(1)}$ and $N_{1}$ to probe intensity dependent TA of a blended F8BT:polystyrene film. As expected, $\sigma_{1 \rightarrow 2}^{(1)}$ is dependent on probe wavelength but independent of excitation density, while the reverse is true for $N_{1}$.

To return to our comparison of fitted $N_{1}$ with values estimated using pump intensity, Table 6.1 presents the results from the $t=500 \mathrm{fs}$ dataset. These values should closely match the intitial excited system, as the kinetics indicate negligible relaxation from $S_{1}$ within $500 \mathrm{fs}$. We can see that the model returns population densities that all seem to exceed our expected value by a factor of $\sim 2.5-3$. While this could be due to probe absorption processes that are unaccounted for in our 


\begin{tabular}{|c|c|c|c|c|c|c|}
\hline \multirow[b]{2}{*}{ F8BT } & \multicolumn{2}{|c|}{$\begin{array}{c}\text { Expected } N_{1} \\
/ 10^{17} \mathrm{~cm}^{-3}\end{array}$} & \multicolumn{2}{|c|}{$\begin{array}{c}N_{1}, t=500 \mathrm{fs} \\
/ 10^{17} \mathrm{~cm}^{-3}\end{array}$} & \multicolumn{2}{|c|}{$\begin{array}{c}\sigma_{1 \rightarrow 2}^{(1)}, t=500 \mathrm{fs} \\
/ 10^{-16} \mathrm{~cm}^{2}\end{array}$} \\
\hline & $1120 \mu \mathrm{m}$ & $680 \mu \mathrm{m}$ & $800 \mathrm{~nm}$ & $900 \mathrm{~nm}$ & $800 \mathrm{~nm}$ & $900 \mathrm{~nm}$ \\
\hline $10 \mu \mathrm{J} \mathrm{cm}^{-2}$ & 1.98 & 5.37 & 6.54 & 5.26 & 1.44 & 4.03 \\
\hline $20 \mu \mathrm{J} \mathrm{cm}^{-2}$ & 3.96 & 10.7 & 9.64 & 10.3 & 2.12 & 4.21 \\
\hline F8BT:PS & $1120 \mu \mathrm{m}$ & $680 \mu \mathrm{m}$ & $800 \mathrm{~nm}$ & $900 \mathrm{~nm}$ & $800 \mathrm{~nm}$ & $900 \mathrm{~nm}$ \\
\hline $10 \mu \mathrm{J} \mathrm{cm}^{-2}$ & 0.626 & 1.70 & 1.57 & 1.27 & 1.50 & 3.67 \\
\hline $20 \mu \mathrm{J} \mathrm{cm}^{-2}$ & 1.25 & 3.40 & 3.81 & 3.74 & 1.31 & 3.02 \\
\hline
\end{tabular}

Table 6.1: Fitted parameters for neat F8BT film $(l=705 \mathrm{~nm}, A=1.82 @ 400 \mathrm{~nm})$ and F8BT:PS film ( $l=1440 \mathrm{~nm}, A=0.44 @ 400 \mathrm{~nm})$. The "Expected $N_{1}$ " value is an estimate of initial excitation density based on pump power and film optical density and thickness.

model, it is surprising that by all other measures the fitted parameters behave exactly as expected in response to changes in experimental configuration.

Another possibility is that our measurement of pump spot size is not correct. Indeed, the very large spot used does not fit entirely on the webcam CCD element that we use to measure beam widths. Table 6.1 includes a second column of esimated initial population densities, assuming a pump FWHM of $680 \mu \mathrm{m}$. The resulting densities are in good agreement with all of the fitted population densities.

\subsubsection{Two-photon absorption by excited states}

We will now consider several of the transitions shown in Figure 6.5 that have not, to this point, been included in our numerical model of probe absorption.

Two-photon absorption from the excited state $\mathrm{S}_{1}$ occurs with rate $\frac{N_{1}(n, r, t) I^{2}(r, z, t) \sigma_{1 \rightarrow 2}^{(2)}}{2 \hbar \omega}$ given by Equation 6.3.1. The non-linear increase in absorption with probe intensity serves to increase $\Delta T / T$ over a certain range of probe intensity, before $N_{1}$ is sufficiently depleted for saturation to cause the previously described reduction in signal amplitude.

The two photon cross section from an excited state is particularly difficult to determine, and will be strongly dependent on probe wavelength. In particular, if the energy of two probe photons is enough to ionize any excitations from $S_{1}$, the continuum of states available to those ionized electrons could reasonably be 
expected to result in a particularly large two photon absorption cross-section (i.e. oscillator strength).

If we approximate the energy of electrons in the $S_{1}$ state as being equal to the LUMO energy in our polymer $(-3.3 \mathrm{eV}$ in F8BT $[49,114,190])$ minus $0.25 \mathrm{eV}$ to account for exciton binding energy, then the onset for this strong two photon absorption should be at energies above $1.78 \mathrm{eV}$ (i.e., at wavelengths shorter than $697 \mathrm{~nm})$.

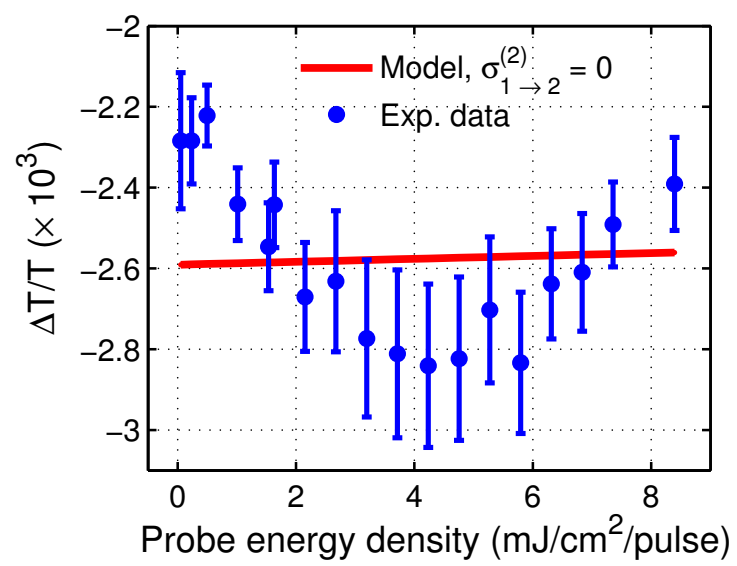

(a)

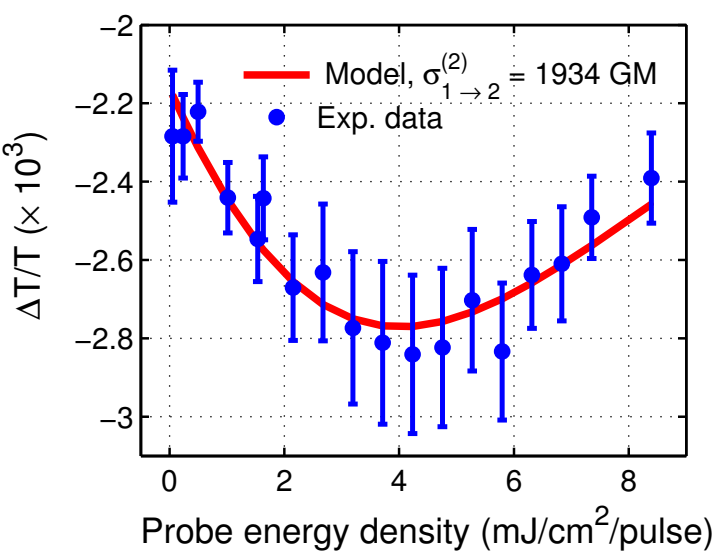

(b)

Figure 6.10: TA pumped at $400 \mathrm{~nm}\left(45 \mu \mathrm{J} \mathrm{cm}^{-2}\right.$ pulse $\left.^{-1}\right)$. We see that at higher probe energies (in this case, $700 \mathrm{~nm}$ ), two-photon absorption of the probe becomes significant. The two-photon cross section $\sigma_{(1 \rightarrow 2)}^{(2)}$ is set to 0 in $6.10 \mathrm{a}$, but was left as a free parameter in $6.10 \mathrm{~b}$, where fitted values were $\sigma_{1 \rightarrow 2}^{(1)}=1.11 \times 10^{-16} \mathrm{~cm}^{2}$, $\sigma_{(1 \rightarrow 2)}^{(2)}=1930 \mathrm{GM}, N_{1, \text { mean }}=2.64 \times 10^{17} \mathrm{~cm}^{-3}$.

We collected probe intensity dependent TA of a neat F8BT film $(750 \mathrm{~nm}$ thick, $A=1.88$ at $400 \mathrm{~nm}$ ) with a $700 \mathrm{~nm}$ probe, shown in Figure 6.10a. As predicted, there is an initial increase in signal amplitude with probe intensity, a trend that is absent in the data from the longer wavelength probes, and that the model is unable to reproduce when $\sigma_{1 \rightarrow 2}^{(2)}=0$. When the two photon cross section is included as a third free parameter in our model the trend is well fit by the data. The curve in $6.10 \mathrm{~b}$ is the predicted probe dependence with fitted parameters $N_{1}=$ $3.6 \times 10^{18} \mathrm{~cm}^{-3}, \sigma_{1 \rightarrow 2}^{(1)}=2.3 \times 10^{-17} \mathrm{~cm}^{2}$ and $\sigma_{1 \rightarrow 2}^{(2)}=1934 \mathrm{GM}^{\text {iii }}$. It is difficult to know whether this represents a realistic value of the two photon cross section for

$$
\text { iii } 1 \mathrm{GM}=10^{-50} \mathrm{~cm}^{4} \text { s photon }^{-1}[191]
$$


$\mathrm{S}_{1}$, as a search of the literature did not return any values of $\sigma_{1 \rightarrow 2}^{(2)}$ for semiconducting polymers ${ }^{\mathrm{iv}}$. However it is clear that the inclusion of this effect was crucial for reproducing the observed trend.

\subsubsection{Placing boundaries on sequential absorption effects}

Single photon absorption from $\mathrm{S}_{2}$ occurs from $\mathrm{S}_{2}$ with rate $\frac{N_{2}(n, r, t) I(r, z, t) \sigma_{2 \rightarrow n}^{(1)}}{\hbar \omega}$. Like the other transitions, it represents a potential source of error for our model. Similar to $\sigma_{1 \rightarrow 2}^{(2)}, \sigma_{2 \rightarrow n}^{(1)}$ will increase the amplitude of our $\Delta T / T$ signal by allowing more than one probe photon to be absorbed per initial pump excitation. Of course, the process is limited by the population density of $S_{2}$, which is itself populated by the probe pulse. Absorption by excited states in $S_{2}$ can therefore be considered a sequential absorption process (see [188]).

In order to determine the degree to which this process contributes to overall probe absorption, we conducted pump-push-probe spectroscopy of a neat F8BT film. In this experiment, an initial pump pulse at $400 \mathrm{~nm}$ (identical to the one used in the previous sections) is followed by an intense $800 \mathrm{~nm}$ 'push' pulse that drives excitations from $S_{1}$ to $S_{2}$, determining the population density $N_{2}$ (replicating the effect of the probe pulse in our previous experiments). Finally a broadband probe (NOPA, $550-850 \mathrm{~nm}$ ) is used to measure the absorption of the system. By chopping the pump at $f / 2$ and the push at $f / 4$ (where $f$ is the repetition rate of our laser), we can reject the absorption of our probe due to excitations directly from the pump, and determine the contribution $\Delta(\Delta T / T)$ due exclusively to the effect of the push pulse on $N_{1}$, given by

$$
\Delta\left(\frac{\Delta T}{T}\right)=\frac{\left(\frac{\Delta T}{T}\right)_{\text {pushed }}-\left(\frac{\Delta T}{T}\right)}{\frac{\Delta T}{T}}-\left(\frac{\Delta T}{T}\right)_{\text {pump off, pushed }},
$$

where the final term is a background subtraction to remove any signal due to the probe acting directly on the ground state via two photon absorption (i.e. the $\sigma_{0 \rightarrow 1}^{(2)}$ transition).

Figure 6.11a shows a map of $\Delta(\Delta T / T)$ for a blended F8BT:polystyrene film where the arrival time of the push pulse at the sample was fixed with respect

\footnotetext{
${ }^{\text {iv }}$ To give at least one point of reference, ground state two-photon cross sections as high as $\sigma_{0 \rightarrow 1}^{(2)}=$ 3700 GM have been measured in centrosymmetric donor-acceptor-donor molecules optimised for TPA [192].
} 
to the pump. The delay time stated is therefore push-probe delay, with kinetics of the main features presented in Figure 6.11b. The pump energy density was $42 \mu \mathrm{J} \mathrm{cm}^{-2}$ pulse $^{-1}$, while the push power delivered approximately the same energy density to the sample as the highest probe intensity used in Section 6.4 $\left(\sim 1 \mathrm{~mJ} \mathrm{~cm}^{-2}\right.$ pulse $\left.^{-1}\right)$.

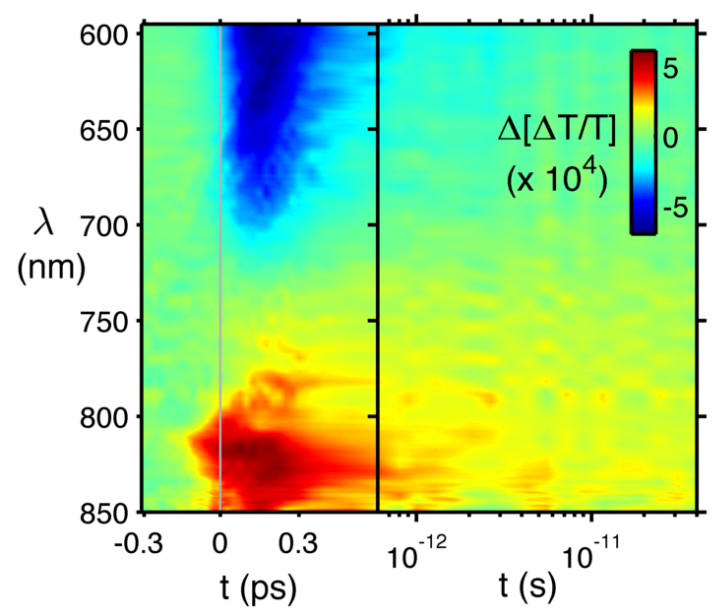

(a)

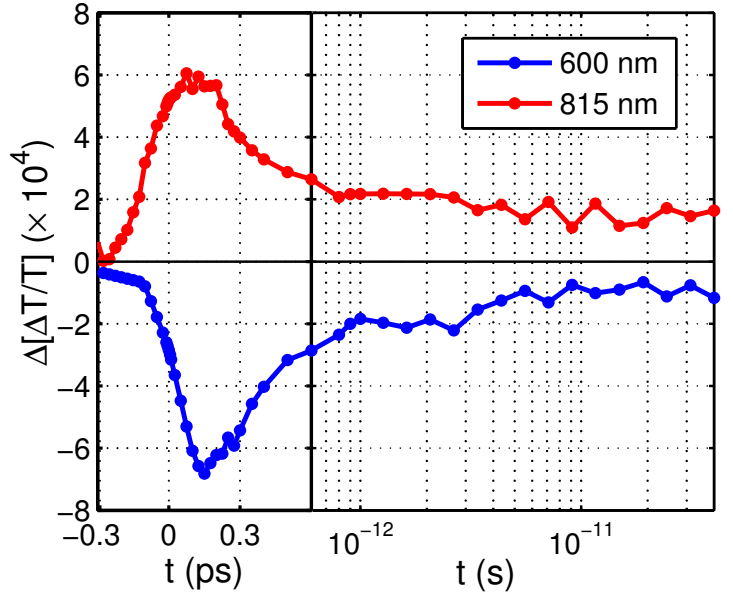

(b)

Figure 6.11: $\left(\Delta\left(\frac{\Delta T}{T}\right)\right)$ Pump-push-broadband probe spectroscopy of F8BT:PS. The positive signal at our wavelengths of interest (800 and $850 \mathrm{~nm}$ ) shows that $P_{(2 \rightarrow n)}<P_{(1 \rightarrow 2)}$.

We see an initial fast decay taking place within our instrument response time ( $\sim 200 \mathrm{fs}$ ) followed by a long lived feature that is constant over the 40 ps window of the experiment. The signal has a peak magnitude of $6 \times 10^{-4}$ and a spectral shape that is effectively an inverted version of the standard TA spectra presented in Figure 6.3a, indicating that the effect of the push pulse here is a decrease in photoinduced absorption - and therefore of the magnitude of the standard TA signal (which itself had had a peak magnitude of $11 \times 10^{-3}$ ).

From this we can infer that the contribution of the $\sigma_{2 \rightarrow n}^{(1)}$ transition to absorption of the probe is significantly less than that from the $\sigma_{1 \rightarrow 2}^{(1)}$ transition. In fact, we have a good idea of the relative fraction of excitations in $S_{1}$ and $S_{2}$ from our previous calculations, (as illustrated in the lower panel of 6.7), where under the most intense probe intensities the mean fraction of total excitations in $S_{2}$ throughout the sample was $N_{2, \text { final }} / N_{1, \text { initial }} \approx 0.79 \times 10^{17} / 3.8 \times 10^{17}=20 \%$. We can therefore make a ballpark estimate of $\sigma_{2 \rightarrow n}^{(1)} \approx \frac{1}{0.2} \frac{6 \times 10^{-4}}{11 \times 10^{-3}} \sigma_{1 \rightarrow 2}^{(1)}=0.4 \times 10^{-16} \mathrm{~cm}^{2}$ using our previously 
fitted value of $\sigma_{1 \rightarrow 2}^{(1)}$ as a reference. Plugging this cross section back into the model and recalculating the fits presented in Table 6.1 results in a reduction in fitted $N_{1}$ of $\sim 25 \%$.

We attribute the remnant signal extending beyond $10 \mathrm{ps}$ to charge pairs generated via excitation to higher lying singlet states, as has been shown to occur due to singlet-singlet annihilation in F8BT [119] and as a result of intense push pulses in other neat polymers [193].

We can conclude that sequential absorption by excitations in the $S_{2}$ state contributes an appreciable effect to the probe intensity dependence of our system. However, the effect is insufficient to explain the threefold discrepancy between the estimated and fitted values of $N_{1}$ shown in Table 6.1, which would require an $\mathrm{S}_{2}$ cross section $\sigma_{2 \rightarrow n}^{(1)} \approx 3 \times 10^{-16} \mathrm{~cm}^{2}$.

\subsection{Single-shot probe intensity dependence}

The use of probe intensity dependent TA holds promise as a method for quantifying previously qualitative spectroscopic measurements, and for elucidating the interplay between competing absorptive processes in semiconducing materials. The main experimental challenges for the technique relate to the neccessary stability and repeatability of the pump and probe light, as well as the large dataset required, both of which result in this method being rather time-costly.

Both of these issues could be addressed by recognising that a single laser pulse exhibits a range of intensities, with a well defined spatial distribution (i.e. gaussian with $r$ ) that could be harnessed to enable 'single-shot' probe saturation spectroscopy. Such an experimental configuration would be identical to the one described above, with the exception that the region of interaction in the sample would be imaged (via telescopic optics) directly on to the detector array, rather than through a polychromator. The pixels of the array would then each be measuring TA on a different point in the sample, with those points each experiencing a different probe intensity based on their distance from the center of the optical axis.

Figure 6.12 shows the spatial dependence of measured $\Delta T / T$ in the plane of the sample calculated for two probe intensities using the probe absorption model described in Section 6.3.2. In this calculation, as with our experiment, the pump spot is much larger $(\mathrm{FWHM}=1120 \mu \mathrm{m})$ than our probe $(\mathrm{FWHM}=150 \mu \mathrm{m})$. We can see that at the low probe power, the distribution of $\Delta T / T$ is determined 

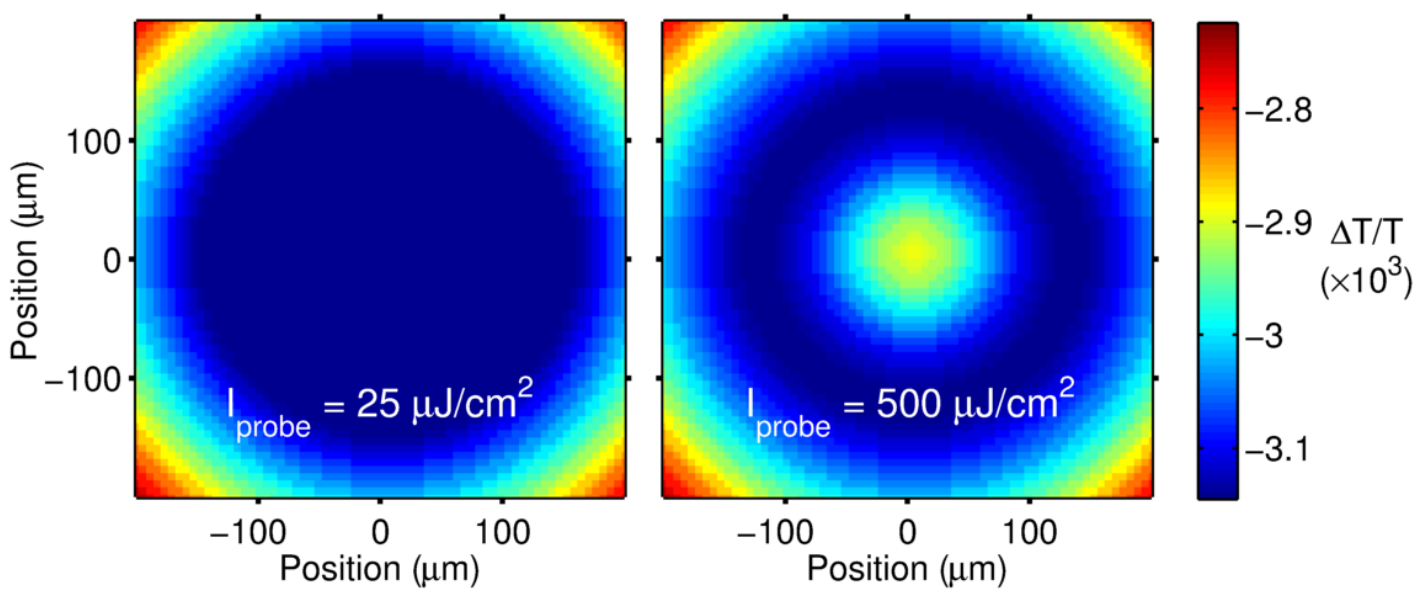

Figure 6.12: Map of probed $\Delta T / T$ values as a function of position on the incident face of the sample.

exclusively by the shape of the pump pulse. At high probe intensity, the probe sufficientyl depletes $N_{1}$ near the center of the beam to cause a significant reduction in $\Delta T / T$. If a strip of this probe light was then imaged on to a linear array, a spatially dependent $\Delta T / T$ curve such as that in Figure 6.13 could be detected which would be characteristic of $N_{1}$ and $\sigma_{1 \rightarrow 2}^{(1)}$ for that sample.

The collection of all required probe intensities simultaneously would eliminate the experimental challenges of laser and sample stability, and of collection time. The main complication with the single shot method - and the reason why we have not implemented it ourselves - is the need to collect probe light of a wide range of intensities while somehow keeping the count on each pixel within the useable dynamic range of the digitization circuitry. The most likely solution to this is to use a graduated attenuator matched to the distribution of probe intensities. 


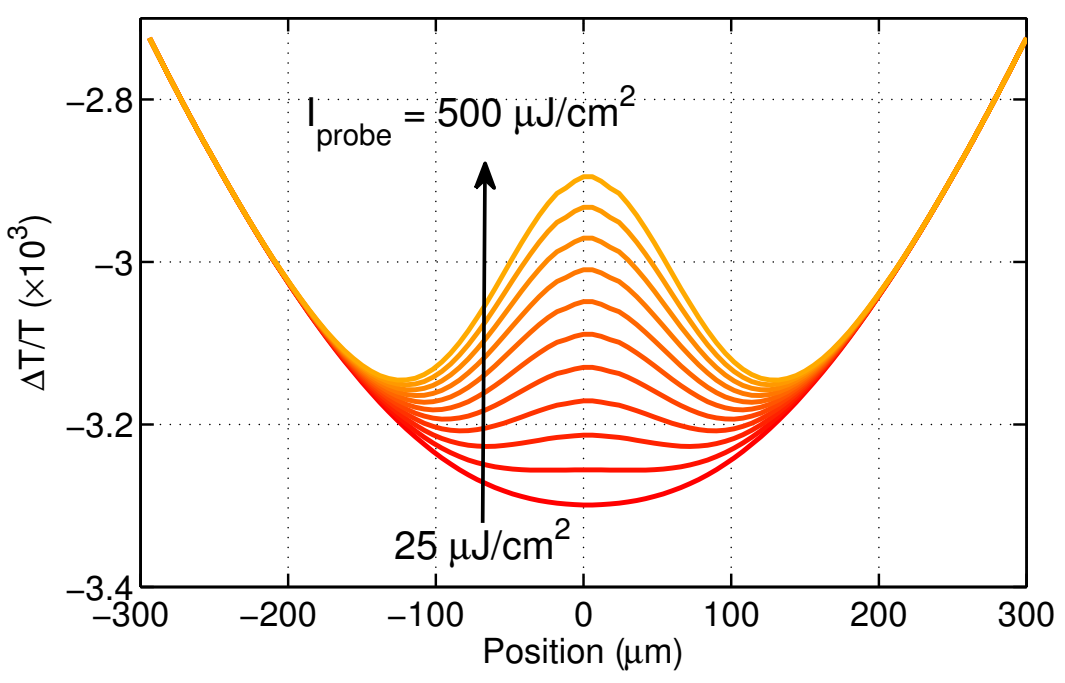

Figure 6.13: Single shot spatially resolved transient-absorption spectroscopy at a range of probe pulse energies. At high probe energy greater saturation of the signal occurs in the more intense regions of the probe pulse.

\subsection{Conclusions}

We have demonstrated that the use of intense probe pulses in TA spectroscopy may drive a sufficient fraction of the absorbing species to a higher state that subsequent probe absorption is reduced. As a result, the Beer-Lambert approximation fails to predict the magnitude of absorption by excited states, resulting in a decoupling of excited state cross-section and population density. We were able to detect this effect in the conjugated polymer F8BT by the use of highly stable and sensitive spectroscopy techniques.

A numerical model was developed that predicts probe absorption under high intensities, and was fitted to the measured probe-dependent TA data, returning values of the excited state $\mathrm{S}_{1} \rightarrow \mathrm{S}_{2}$ single photon aborption cross-section $\sigma_{1 \rightarrow 2}^{(1)}=$ $1.6 \times 10^{-16} \mathrm{~cm}^{2} \pm 40 \%$ at $800 \mathrm{~nm}$, and $3.7 \times 10^{-16} \mathrm{~cm}^{2} \pm 30 \%$ at $900 \mathrm{~nm}$, where the stated errors represent two standard deviations of the values determined over several measurements.

The effect of absorption by other processes was discussed, and shown by pump-push-probe spectroscopy to be a negligible effect at the wavelengths of interest, though significant two-photon absorption occured when probing at shorter wavelengths. 
The method currently requires time consuming and repetitive measurements, and the implementation of a single-shot probe intensity-dependent experiment was described. The technique may be useful for quantifying salient parameters in many systems, such as branching yields or cross-sections required for photophysical models. 


\section{Chapter 7}

\section{Summary \& future work}

Organic photovoltaics are an emerging technology with the potential to provide a widespread and low cost source of solar energy. Despite the significant progress made over the last decade, improvements in power conversion efficiency are still required for their implementation to take place on a wide scale.

Understanding the process by which tightly bound charge pairs overcome their mutual attraction is crucial to guiding the design of the next generation of OPVs. The process represents an interesting puzzle, as traditional theories fail to explain the rate and efficiency of charge separation that is observed to take place in many of these materials. An emerging set of theories invoke delocalization of primary excitations on ultrafast timescales that are able to couple to the manifold of excited charge-transfer states, enabling sufficient separation to overcome the Coulombic binding energy. A central feature of this model is a branching of photogenerated charges on ultrafast timescales into bound and well separated populations. Measurement of key parameters in this process will enable further development of our understanding, and guide the design of more efficient materials.

Chapter 2 of this thesis described the design and construction of an ultrasensitive transient absorption spectrometer capable of invesigating excited state photophysics in thin films over a continuous window from 100 fs to arbitrarily long time scales. In Chapter 3 we undertook an analysis of the sources of noise in TA spectroscopy, and introduced a novel and counter-intuitive application of highly-chirped broadband pulses that effectively resulted in a better than fivefold improvement in sensitivity simply through the addition of a glass block in the beam path. The technique was tested by measuring the kinetics of charge formation in P3HT:PCBM in a 30 minute lab session with sensitivity in differential 
transmission of approximately $3 \times 10^{-6}$. Future work in the lab mainly involves expanding our measurement window to different energies (i.e. further into the infrared), and to shorter times through the construction of an optical compressor.

In Chapter 4 we presented broadband TA of a range of OPV blends of varying performance, generating a library of samples amongst which to compare results from several spectroscopic techniques. The dependence of recombination rate on intensity was used to measure the yield of well separated charges, which provides a better indication of device efficiency than simple reocombination measurements such as charge lifetime. Finally, we briefly discussed the reconciliation of electronic and optical measurements of recombination, resulting in a combined technique capable of resolving charge recombination over an exceptional range of timescales $\left(10^{-13}-10^{-2} \mathrm{~s}\right)$ and charge densities $\left(10^{13}-10^{19} \mathrm{~cm}^{-3}\right.$ and above).

In Chapter 5 we showed that we were able to access a regime at low temperature where all charges are effectively frozen out after the charge generation process, and forced to recombine geminately by tunneling. The sensitive dependence of tunneling rate on distance provides us with a useful ruler with which to measure the separation of charges immediately following separation. The rates at which tunneling recombination takes place turn out to be distributed bimodally, with the relative population in each node strongly correlated with the free charge fractions measured in Chapter 4 . When taking reasonable values for the distance dependence $\beta$ of tunneling, this suggests a critical separation for free charge generation of $3-4 \mathrm{~nm}$.

Expansion of this work would involve measurements of tunneling recombination at different energies, particularly in the infrared where the different subpopulations may be able to be individually targeted. Measurements to rule out or confirm the possible influence of triplet excitons, particularly in the roomtemperature recombination measurements, would be useful. This could potentially be done via broadband infrared TA, [40,101] or THz spectroscopy. [17] It would also be fascinating to employ a reexcitation pulse, to see if the pathways for charge separation could be re-accessed, and if this could be detected in a redistribution of tunneling recombination rates.

Finally, in Chapter 6 we presented the use of probe intensity-dependent spectroscopy, in which the time-resolved excited state absorption cross-section and population density can be measured. We find the single photon aborption crosssection of the first singlet excited state in F8BT to be $\sigma_{1 \rightarrow 2}^{(1)}=1.6 \times 10^{-16} \mathrm{~cm}^{2} \pm 40 \%$ 
at $800 \mathrm{~nm}$, and $3.7 \times 10^{-16} \mathrm{~cm}^{2} \pm 30 \%$ at $900 \mathrm{~nm}$, where the stated errors represent two standard deviations of the values determined over several measurements. This technique could prove extremely useful in quantifying branching yields, for example in materials exhibiting singlet fission or triplet formation, or even in illuminating the role played by ultrafast delocalization in free charge photogeneration.

Further work must be done to make the probe saturation measurement practical, as the long and repetitive measurements in its current form are an impediment and source of error. A single-shot probe saturation method has been proposed, and could be implemented with a small amount of specialized optics. The real acid test for the method would come when applying it to systems with more than one excited state species - for example in charge generating blends. 


\section{Bibliography}

[1] U. E. I. Administration, “International Energy Outlook 2013,” tech. rep., 2013.

[2] IPCC, Climate Change 2007 - Mitigation: Working Group III Contribution to the Fourth Assessment Report of the IPCC. 2007.

[3] IPCC, Climate Change 2007 - The Physical Science Basis: Working Group I Contribution to the Fourth Assessment Report of the IPCC. 2007.

[4] N. S. Lewis, "Toward cost-effective solar energy use.," Science, vol. 315, pp. 798-801, Feb. 2007.

[5] V. Devabhaktuni, M. Alam, S. Shekara Sreenadh Reddy Depuru, R. C. Green, D. Nims, and C. Near, "Solar energy: Trends and enabling technologies," Renewable and Sustainable Energy Reviews, vol. 19, pp. 555-564, Mar. 2013.

[6] G. Masson, S. Orlandi, and M. Rekinger, "Global Market Outlook for Photovoltaics," tech. rep., European Photovoltaic Industry Association, 2014.

[7] CIA, World Factbook.

[8] B. Parida, S. Iniyan, and R. Goic, "A review of solar photovoltaic technologies," Renewable and Sustainable Energy Reviews, vol. 15, pp. 1625-1636, Apr. 2011.

[9] B. O'Regan and M. Grätzel, "A low-cost, high-efficiency solar cell based on dye-sensitized colloidal TiO2 films," Nature, vol. 353, pp. 737-740, Oct. 1991.

[10] M. Grätzel, “Photoelectrochemical cells.," Nature, vol. 414, pp. 338-44, Nov. 2001.

[11] M. M. Lee, J. Teuscher, T. Miyasaka, T. N. Murakami, and H. J. Snaith, "Efficient hybrid solar cells based on meso-superstructured organometal halide perovskites.," Science, vol. 338, pp. 643-7, Nov. 2012.

[12] H. J. Snaith, "Perovskites: The Emergence of a New Era for Low-Cost, High-Efficiency Solar Cells," The Journal of Physical Chemistry Letters, vol. 4, pp. 3623-3630, Nov. 2013.

[13] F. C. Krebs, S. a. Gevorgyan, and J. Alstrup, "A roll-to-roll process to flexible polymer solar cells: model studies, manufacture and operational stability studies," Journal of Materials Chemistry, vol. 19, no. 30, p. 5442, 2009.

[14] Y. Liang, Z. Xu, J. Xia, S.-T. Tsai, Y. Wu, G. Li, C. Ray, and L. Yu, “For the bright future-bulk heterojunction polymer solar cells with power conversion efficiency of 7.4\%.," Advanced materials (Deerfield Beach, Fla.), vol. 22, pp. E135-8, May 2010. 
[15] R. F. Service, "Solar energy. Outlook brightens for plastic solar cells.," Science, vol. 332, p. 293, Apr. 2011.

[16] "Heliatek GmbH." http: / / www . heliatek. com/? lang=en, 2014.

[17] A. Köhler and H. Bässler, "What controls triplet exciton transfer in organic semiconductors?," Journal of Materials Chemistry, vol. 21, no. 12, p. 4003, 2011.

[18] N. W. Ashcroft and N. D. Mermin, Solid State Physics, vol. 2. Holt, Rinehart and Winston, 1976.

[19] N. C. Giebink, G. P. Wiederrecht, M. R. Wasielewski, and S. R. Forrest, "Thermodynamic efficiency limit of excitonic solar cells," Physical Review B, vol. 83, p. 195326, May 2011.

[20] M. Kasha, H. R. Rawls, and M. Ashraf El-Bayoumi, "The exciton model in molecular spectroscopy," Pure and Applied Chemistry, vol. 11, pp. 371-392, Jan. 1965.

[21] J.-L. Brédas, J. Cornil, and A. J. Heeger, "The exciton binding energy in luminescent conjugated polymers," Advanced Materials, vol. 8, pp. 447-452, May 1996.

[22] G. D. Scholes and G. Rumbles, "Excitons in nanoscale systems.," Nature materials, vol. 5, pp. 683-96, Sept. 2006.

[23] D. Beljonne, J. Cornil, R. H. Friend, R. A. J. Janssen, and J. L. Brédas, “Influence of Chain Length and Derivatization on the Lowest Singlet and Triplet States and Intersystem Crossing in Oligothiophenes," Journal of the American Chemical Society, vol. 118, pp. 6453-6461, Jan. 1996.

[24] E. Collini and G. D. Scholes, "Coherent intrachain energy migration in a conjugated polymer at room temperature.," Science, vol. 323, pp. 369-73, Jan. 2009.

[25] F. Dubin, R. Melet, T. Barisien, R. Grousson, L. Legrand, M. Schott, and V. Voliotis, “Macroscopic coherence of a single exciton state in an organic quantum wire," Nature Physics, vol. 2, pp. 32-35, Dec. 2005.

[26] R. Williams and K. Song, "The self-trapped exciton," Journal of Physics and Chemistry of Solids, vol. 51, pp. 679-716, Jan. 1990.

[27] J.-L. Brédas, D. Beljonne, V. Coropceanu, and J. Cornil, “Charge-transfer and energy-transfer processes in pi-conjugated oligomers and polymers: a molecular picture.," Chemical reviews, vol. 104, pp. 4971-5004, Nov. 2004.

[28] H. Bässler and A. Köhler, "Charge transport in organic semiconductors.," Topics in current chemistry, vol. 312, pp. 1-65, Jan. 2012.

[29] S. Athanasopoulos, S. T. Hoffmann, H. Bässler, A. Köhler, and D. Beljonne, “To Hop or Not to Hop? Understanding the Temperature Dependence of Spectral Diffusion in Organic Semiconductors," The Journal of Physical Chemistry Letters, vol. 4, pp. 1694-1700, May 2013.

[30] L. C. Groff, X. Wang, and J. D. McNeill, “Measurement of Exciton Transport in Conjugated Polymer Nanoparticles," The Journal of Physical Chemistry C, vol. 117, pp. 25748-25755, Dec. 2013. 
[31] B. J. Schwartz, T.-q. Nguyen, J. Wu, and S. H. Tolbert, "Interchain and intrachain exciton transport in conjugated polymers: ultrafast studies of energy migration in aligned MEHPPV/mesoporous silica composites," Synthetic Metals, vol. 116, pp. 35-40, Jan. 2001.

[32] R. R. Lunt, N. C. Giebink, A. a. Belak, J. B. Benziger, and S. R. Forrest, “Exciton diffusion lengths of organic semiconductor thin films measured by spectrally resolved photoluminescence quenching," Journal of Applied Physics, vol. 105, no. 5, p. 053711, 2009.

[33] O. V. Mikhnenko, F. Cordella, a. B. Sieval, J. C. Hummelen, P. W. M. Blom, and M. a. Loi, "Temperature dependence of exciton diffusion in conjugated polymers.," The journal of physical chemistry. B, vol. 112, pp. 11601-4, Sept. 2008.

[34] D. E. Markov, E. Amsterdam, P. W. M. Blom, A. B. Sieval, and J. C. Hummelen, “Accurate measurement of the exciton diffusion length in a conjugated polymer using a heterostructure with a side-chain cross-linked fullerene layer.," The journal of physical chemistry. A, vol. 109, pp. 5266-74, June 2005.

[35] S. Gélinas, A. Rao, A. Kumar, S. L. Smith, A. W. Chin, J. Clark, T. S. van der Poll, G. C. Bazan, and R. H. Friend, "Ultrafast long-range charge separation in organic semiconductor photovoltaic diodes.," Science, vol. 343, pp. 512-6, Jan. 2014.

[36] M. D. McGehee and A. J. Heeger, "Semiconducting (Conjugated) Polymers as Materials for Solid-State Lasers," Advanced Materials, vol. 12, pp. 1655-1668, Nov. 2000.

[37] T. M. Clarke and J. R. Durrant, “Charge photogeneration in organic solar cells.," Chemical reviews, vol. 110, pp. 6736-67, Nov. 2010.

[38] P. J. Jadhav, P. R. Brown, N. Thompson, B. Wunsch, A. Mohanty, S. R. Yost, E. Hontz, T. Van Voorhis, M. G. Bawendi, V. Bulović, and M. a. Baldo, "Triplet exciton dissociation in singlet exciton fission photovoltaics.," Advanced Materials, vol. 24, pp. 6169-74, Dec. 2012.

[39] B. J. Walker, A. J. Musser, D. Beljonne, and R. H. Friend, "Singlet exciton fission in solution," Nature Chemistry, Nov. 2013.

[40] P. C. Y. Chow, S. Gélinas, A. Rao, and R. H. Friend, "Quantitative bimolecular recombination in organic photovoltaics through triplet exciton formation.," Journal of the American Chemical Society, vol. 136, pp. 3424-9, Mar. 2014.

[41] A. Rao, P. C. Y. Chow, S. Gélinas, C. W. Schlenker, C.-Z. Li, H.-L. Yip, A. K.-Y. Jen, D. S. Ginger, and R. H. Friend, "The role of spin in the kinetic control of recombination in organic photovoltaics," Nature, pp. 6-11, Aug. 2013.

[42] C.-L. Lee, X. Yang, and N. Greenham, "Determination of the triplet excited-state absorption cross section in a polyfluorene by energy transfer from a phosphorescent metal complex," Physical Review B, vol. 76, p. 245201, Dec. 2007.

[43] C. W. Tang and S. A. Vanslyke, "Organic electroluminescent diodes," Applied Physics Letters, vol. 51, p. 913, 1987. 
[44] J. H. Burroughes, D. D. C. Bradley, A. R. Brown, R. N. Marks, K. Mackay, R. H. Friend, P. L. Burns, and A. B. Holmes, "Light-emitting diodes based on conjugated polymers," Nature, vol. 347, pp. 539-541, Oct. 1990.

[45] R. H. Friend, R. W. Gymer, A. B. Holmes, J. H. Burroughes, R. Marks, C. Taliani, D. D. C. Bradley, D. A. Dos Santos, J. L. Bredas, M. Lo, W. R. Salaneck, M. Logdlund, W. R. Salaneck, J. L. Bre, and V. Slyke, "Electroluminescence in conjugated polymers," Nature, vol. 397, pp. 121-128, 1999.

[46] J. H. Burroughes, C. A. Jones, and R. H. Friend, "New semiconductor device physics in polymer diodes and transistors," Nature, vol. 335, pp. 137-141, Sept. 1988.

[47] H. Sirringhaus, P. J. Brown, R. H. Friend, M. M. Nielsen, K. Bechgaard, B. M. W. LangeveldVoss, A. J. H. Spiering, R. A. J. Janssen, E. W. Meijer, P. Herwig, and D. M. de Leeuw, "Two-dimensional charge transport in self-organized, high-mobility conjugated polymers," Nature, vol. 401, pp. 685-688, Oct. 1999.

[48] H. Sirringhaus, T. Kawase, R. H. Friend, T. Shimoda, M. Inbasekaran, W. Wu, and E. P. Woo, "High-resolution inkjet printing of all-polymer transistor circuits.," Science, vol. 290, pp. 2123-6, Dec. 2000.

[49] L.-1. Chua, J. Zaumseil, J.-f. Chang, E. C.-W. Ou, P. K.-H. Ho, H. Sirringhaus, and R. H. Friend, "General observation of n-type field-effect behaviour in organic semiconductors.," Nature, vol. 434, pp. 194-9, Mar. 2005.

[50] D. Moses, "High quantum efficiency luminescence from a conducting polymer in solution: A novel polymer laser dye," Applied Physics Letters, vol. 60, no. 26, p. 3215, 1992.

[51] W. Graupner, G. Leising, G. Lanzani, M. Nisoli, De Silvestri S, and U. Scherf, “Femtosecond relaxation of photoexcitations in a poly(para-phenylene)-type ladder polymer.," Physical review letters, vol. 76, pp. 847-850, Jan. 1996.

[52] R. Gupta, M. Stevenson, A. Dogariu, M. D. McGehee, J. Y. Park, V. Srdanov, A. J. Heeger, 
[57] G. Yu, J. Gao, J. C. Hummelen, F. Wudl, and A. J. Heeger, "Polymer Photovoltaic Cells: Enhanced Efficiencies via a Network of Internal Donor-Acceptor Heterojunctions," Science, vol. 270, pp. 1789-1791, Dec. 1995.

[58] S. R. Cowan, N. Banerji, W. L. Leong, and A. J. Heeger, "Charge Formation, Recombination, and Sweep-Out Dynamics in Organic Solar Cells," Advanced Functional Materials, vol. 22, pp. 1116-1128, Mar. 2012.

[59] G. Li, R. Zhu, and Y. Yang, "Polymer solar cells," Nature Photonics, vol. 6, pp. 153-161, Feb. 2012.

[60] J. Peet, J. Y. Kim, N. E. Coates, W. L. Ma, D. Moses, a. J. Heeger, and G. C. Bazan, “Efficiency enhancement in low-bandgap polymer solar cells by processing with alkane dithiols.," Nature materials, vol. 6, pp. 497-500, July 2007.

[61] K. Vandewal, K. Tvingstedt, A. Gadisa, O. Inganäs, and J. V. Manca, “On the origin of the open-circuit voltage of polymer-fullerene solar cells.," Nature materials, vol. 8, pp. 904-9, Nov. 2009.

[62] M. Scharber, D. Mühlbacher, M. Koppe, P. Denk, C. Waldauf, a.J. Heeger, and C. Brabec, “Design Rules for Donors in Bulk-Heterojunction Solar CellsTowards 10\% Energy-Conversion Efficiency," Advanced Materials, vol. 18, pp. 789-794, Mar. 2006.

[63] B. Qi and J. Wang, "Fill factor in organic solar cells.," Physical chemistry chemical physics : PCCP, vol. 15, pp. 8972-82, June 2013.

[64] W. Shockley and H. J. Queisser, "Detailed Balance Limit of Efficiency of p-n Junction Solar Cells," Journal of Applied Physics, vol. 32, no. 3, p. 510, 1961.

[65] D. N. Congreve, J. Lee, N. J. Thompson, E. Hontz, S. R. Yost, P. D. Reusswig, M. E. Bahlke, S. Reineke, T. Van Voorhis, and M. A. Baldo, "External quantum efficiency above $100 \%$ in a singlet-exciton-fission-based organic photovoltaic cell.," Science (New York, N.Y.), vol. 340, pp. 334-7, Apr. 2013.

[66] J. de Wild, a. Meijerink, J. Rath, W. van Sark, and R. Schropp, “Towards upconversion for amorphous silicon solar cells," Solar Energy Materials and Solar Cells, vol. 94, pp. 1919-1922, Nov. 2010.

[67] J.-L. Brédas, J. E. Norton, J. Cornil, and V. Coropceanu, “Molecular understanding of organic solar cells: the challenges.," Accounts of chemical research, vol. 42, pp. 1691-9, Nov. 2009.

[68] M. T. Dang, L. Hirsch, and G. Wantz, "P3HT:PCBM, Best Seller in Polymer Photovoltaic Research," Advanced Materials, vol. 23, pp. 3597-3602, Aug. 2011.

[69] K. G. Jespersen, W. J. D. Beenken, Y. Zaushitsyn, A. Yartsev, M. Andersson, T. o. Pullerits, and V. Sundström, "The electronic states of polyfluorene copolymers with alternating donoracceptor units.," The Journal of chemical physics, vol. 121, pp. 12613-7, Dec. 2004.

[70] P. Kohn, Z. Rong, K. H. Scherer, A. Sepe, M. Sommer, P. Müller-Buschbaum, R. H. Friend, U. Steiner, and S. Hüttner, "Crystallization-Induced 10-nm Structure Formation in P3HT/PCBM Blends," Macromolecules, vol. 46, pp. 4002-4013, May 2013. 
[71] A. J. Moulé and K. Meerholz, "Morphology Control in Solution-Processed BulkHeterojunction Solar Cell Mixtures," Advanced Functional Materials, vol. 19, pp. 3028-3036, Oct. 2009.

[72] M. Campoy-Quiles, T. Ferenczi, T. Agostinelli, P. G. Etchegoin, Y. Kim, T. D. Anthopoulos, P. N. Stavrinou, D. D. C. Bradley, and J. Nelson, “Morphology evolution via self-organization and lateral and vertical diffusion in polymer:fullerene solar cell blends.," Nature Materials, vol. 7, no. 2, pp. 158-164, 2008.

[73] K. Tvingstedt, K. Vandewal, A. Gadisa, F. Zhang, J. Manca, and O. Inganäs, “Electroluminescence from charge transfer states in polymer solar cells.," Journal of the American Chemical Society, vol. 131, pp. 11819-24, Aug. 2009.

[74] L. Goris, a. Poruba, L. Hodakova, M. Vanecek, K. Haenen, M. Nesladek, P. Wagner, D. Vanderzande, L. De Schepper, and J. V. Manca, "Observation of the subgap optical absorption in polymer-fullerene blend solar cells," Applied Physics Letters, vol. 88, no. 5, p. 052113, 2006.

[75] Y. Kim, S. Cook, S. M. Tuladhar, S. a. Choulis, J. Nelson, J. R. Durrant, D. D. C. Bradley, M. Giles, I. McCulloch, C.-S. Ha, and M. Ree, "A strong regioregularity effect in selforganizing conjugated polymer films and high-efficiency polythiophene:fullerene solar cells," Nature Materials, vol. 5, pp. 197-203, Feb. 2006.

[76] B. A. Collins, J. R. Tumbleston, and H. Ade, "Miscibility, Crystallinity, and Phase Development in P3HT/PCBM Solar Cells: Toward an Enlightened Understanding of Device Morphology and Stability," The Journal of Physical Chemistry Letters, vol. 2, pp. 3135-3145, Dec. 2011.

[77] R. A. Marsh, J. M. Hodgkiss, S. Albert-Seifried, and R. H. Friend, "Effect of annealing on P3HT:PCBM charge transfer and nanoscale morphology probed by ultrafast spectroscopy.," Nano letters, vol. 10, pp. 923-30, Mar. 2010.

[78] I. A. Howard, J. M. Hodgkiss, X. Zhang, K. R. Kirov, H. A. Bronstein, C. K. Williams, R. H. Friend, S. Westenhoff, and N. C. Greenham, "Charge recombination and exciton annihilation reactions in conjugated polymer blends.," Journal of the American Chemical Society, vol. 132, pp. 328-35, Jan. 2010.

[79] M. M. Alam and S. a. Jenekhe, “Nanolayered Heterojunctions of Donor and Acceptor Conjugated Polymers of Interest in Light Emitting and Photovoltaic Devices: Photoinduced Electron Transfer at Polythiophene/Polyquinoline Interfaces," The Journal of Physical Chemistry B, vol. 105, pp. 2479-2482, Apr. 2001.

[80] A. Morteani, P. Sreearunothai, L. Herz, R. Friend, and C. Silva, "Exciton Regeneration at Polymeric Semiconductor Heterojunctions," Physical Review Letters, vol. 92, p. 247402, June 2004.

[81] A. C. Morteani, R. H. Friend, and C. Silva, "Exciton trapping at heterojunctions in polymer blends.," The Journal of chemical physics, vol. 122, p. 244906, June 2005. 
[82] K. Hasharoni, M. Keshavarz-K., a. Sastre, R. Gonzalez, C. Bellavia-Lund, Y. Greenwald, T. Swager, F. Wudl, and a. J. Heeger, "Near IR photoluminescence in mixed films of conjugated polymers and fullerenes," The Journal of Chemical Physics, vol. 107, no. 7, p. 2308, 1997.

[83] M. Loi, S. Toffanin, M. Muccini, M. Forster, U. Scherf, and M. Scharber, "Charge Transfer Excitons in Bulk Heterojunctions of a Polyfluorene Copolymer and a Fullerene Derivative," Advanced Functional Materials, vol. 17, pp. 2111-2116, Sept. 2007.

[84] J. Benson-Smith, L. Goris, K. Vandewal, K. Haenen, J. Manca, D. Vanderzande, D. Bradley, and J. Nelson, "Formation of a Ground-State Charge-Transfer Complex in Polyfluorene/ /[6,6]-Phenyl-C61 Butyric Acid Methyl Ester (PCBM) Blend Films and Its Role in the Function of Polymer/PCBM Solar Cells," Advanced Functional Materials, vol. 17, pp. 451-457, Feb. 2007.

[85] L. Onsager, "Initial Recombination of Ions," Physical Review, vol. 54, pp. 554-557, Oct. 1938.

[86] C. L. Braun, "Electric field assisted dissociation of charge transfer states as a mechanism of photocarrier production," The Journal of Chemical Physics, vol. 80, no. 9, p. 4157, 1984.

[87] M. Tachiya, "Breakdown of the Onsager theory of geminate ion recombination," The Journal of Chemical Physics, vol. 89, no. 11, p. 6929, 1988.

[88] V. Gulbinas, Y. Zaushitsyn, V. Sundström, D. Hertel, H. Bässler, and A. Yartsev, “Dynamics of the Electric Field-Assisted Charge Carrier Photogeneration in Ladder-Type Poly(ParaPhenylene) at a Low Excitation Intensity," Physical Review Letters, vol. 89, p. 107401, Aug. 2002.

[89] D. Veldman, O. Ipek, S. C. J. Meskers, J. Sweelssen, M. M. Koetse, S. C. Veenstra, J. M. Kroon, S. S. van Bavel, J. Loos, and R. a. J. Janssen, "Compositional and electric field dependence of the dissociation of charge transfer excitons in alternating polyfluorene copolymer/fullerene blends.," Journal of the American Chemical Society, vol. 130, pp. 7721-35, June 2008.

[90] S. Gelinas, O. Pare-Labrosse, C.-N. Brosseau, S. Albert-Seifried, C. R. McNeill, K. R. Kirov, I. A. Howard, R. Leonelli, R. H. Friend, and C. Silva, “The Binding Energy of Charge-Transfer Excitons Localized at Polymeric Semiconductor Heterojunctions," The Journal of Physical Chemistry C, vol. 115, pp. 7114-7119, Apr. 2011.

[91] H. Ohkita, S. Cook, Y. Astuti, W. Duffy, S. Tierney, W. Zhang, M. Heeney, I. McCulloch, J. Nelson, D. D. C. Bradley, and J. R. Durrant, "Charge carrier formation in polythiophene/fullerene blend films studied by transient absorption spectroscopy.," Journal of the American Chemical Society, vol. 130, pp. 3030-42, Mar. 2008.

[92] S. D. Dimitrov, A. a. Bakulin, C. B. Nielsen, B. C. Schroeder, J. Du, H. Bronstein, I. McCulloch, R. H. Friend, and J. R. Durrant, "On the energetic dependence of charge separation in low-band-gap polymer/fullerene blends.," Journal of the American Chemical Society, vol. 134, pp. 18189-92, Nov. 2012. 
[93] P. Parkinson, J. Lloyd-Hughes, M. Johnston, and L. Herz, “Efficient generation of charges via below-gap photoexcitation of polymer-fullerene blend films investigated by terahertz spectroscopy," Physical Review B, vol. 78, p. 115321, Sept. 2008.

[94] J. Lee, K. Vandewal, S. R. Yost, M. E. Bahlke, L. Goris, M. a. Baldo, J. V. Manca, and T. Van Voorhis, "Charge transfer state versus hot exciton dissociation in polymer-fullerene blended solar cells.," Journal of the American Chemical Society, vol. 132, pp. 11878-80, Sept. 2010.

[95] K. Vandewal, S. Albrecht, E. T. Hoke, K. R. Graham, J. Widmer, J. D. Douglas, M. Schubert, W. R. Mateker, J. T. Bloking, G. F. Burkhard, A. Sellinger, J. M. J. Fréchet, A. Amassian, M. K. Riede, M. D. McGehee, D. Neher, and A. Salleo, "Efficient charge generation by relaxed charge-transfer states at organic interfaces.," Nature materials, vol. 13, pp. 63-8, Jan. 2014.

[96] G. Grancini, M. Maiuri, D. Fazzi, A. Petrozza, H.-J. Egelhaaf, D. Brida, G. Cerullo, and G. Lanzani, "Hot exciton dissociation in polymer solar cells.," Nature materials, vol. 12, pp. 29-33, Jan. 2013.

[97] P. E. Shaw, A. Ruseckas, and I. D. W. Samuel, “Exciton Diffusion Measurements in Poly(3hexylthiophene)," Advanced Materials, vol. 20, pp. 3516-3520, July 2008.

[98] K. Chen, A. J. Barker, M. E. Reish, K. C. Gordon, and J. M. Hodgkiss, "Broadband Ultrafast Photoluminescence Spectroscopy Resolves Charge Photogeneration via Delocalized Hot Excitons in Polymer:Fullerene Photovoltaic Blends.," Journal of the American Chemical Society, vol. 135, pp. 18502-12, Dec. 2013.

[99] A. E. Jailaubekov, A. P. Willard, J. R. Tritsch, W.-L. Chan, N. Sai, R. Gearba, L. G. Kaake, K. J. Williams, K. Leung, P. J. Rossky, and X.-Y. Zhu, "Hot charge-transfer excitons set the time limit for charge separation at donor/acceptor interfaces in organic photovoltaics," Nature Materials, vol. 11, pp. 1-8, Dec. 2012.

[100] A. A. Bakulin, A. Rao, V. G. Pavelyev, P. H. M. van Loosdrecht, M. S. Pshenichnikov, D. Niedzialek, J. Cornil, D. Beljonne, and R. H. Friend, "The role of driving energy and delocalized States for charge separation in organic semiconductors.," Science, vol. 335, pp. 1340-4, Mar. 2012.

[101] F. Etzold, I. a. Howard, N. Forler, D. M. Cho, M. Meister, H. Mangold, J. Shu, M. R. Hansen, K. Müllen, and F. Laquai, "The effect of solvent additives on morphology and excited-state dynamics in PCPDTBT:PCBM photovoltaic blends.," Journal of the American Chemical Society, vol. 134, pp. 10569-83, June 2012.

[102] I. A. Howard, R. Mauer, M. Meister, and F. Laquai, "Effect of morphology on ultrafast free carrier generation in polythiophene:fullerene organic solar cells.," Journal of the American Chemical Society, vol. 132, pp. 14866-76, Oct. 2010.

[103] F. Provencher, N. Bérubé, A. W. Parker, G. M. Greetham, M. Towrie, C. Hellmann, M. Côté, N. Stingelin, C. Silva, and S. C. Hayes, “Direct observation of ultrafast long-range charge separation at polymer-fullerene heterojunctions.," Nature communications, vol. 5, p. 4288, Jan. 2014. 
[104] B. M. Savoie, A. Rao, A. a. Bakulin, S. Gelinas, B. Movaghar, R. H. Friend, T. J. Marks, and M. a. Ratner, "Unequal partnership: asymmetric roles of polymeric donor and fullerene acceptor in generating free charge.," Journal of the American Chemical Society, vol. 136, pp. 2876-84, Feb. 2014.

[105] H. M. Heitzer, B. M. Savoie, T. J. Marks, and M. a. Ratner, “Organic Photovoltaics: Elucidating the Ultra-Fast Exciton Dissociation Mechanism in Disordered Materials.," Angewandte Chemie (International ed. in English), pp. 1-6, May 2014.

[106] H. van Eersel, R. A. J. Janssen, and M. Kemerink, "Mechanism for Efficient Photoinduced Charge Separation at Disordered Organic Heterointerfaces," Advanced Functional Materials, vol. 22, pp. 2700-2708, July 2012.

[107] I. A. Howard, F. Etzold, F. Laquai, and M. Kemerink, “Nonequilibrium Charge Dynamics in Organic Solar Cells," Advanced Energy Materials, pp. n/a-n/a, Mar. 2014.

[108] D. Caruso and A. Troisi, "Long-range exciton dissociation in organic solar cells.," Proceedings of the National Academy of Sciences of the United States of America, vol. 109, pp. 13498-502, Aug. 2012.

[109] J. M. Hodgkiss, S. Albert-Seifried, A. Rao, A. J. Barker, A. R. Campbell, R. A. Marsh, and R. H. Friend, "Exciton-Charge Annihilation in Organic Semiconductor Films," Advanced Functional Materials, vol. 22, pp. 1567-1577, Apr. 2012.

[110] K. Chen, J. K. Gallaher, A. J. Barker, and J. M. Hodgkiss, “Transient Grating Photoluminescence Spectroscopy: An Ultrafast Method of Gating Broadband Spectra," The Journal of Physical Chemistry Letters, vol. 5, pp. 1732-1737, May 2014.

[111] J. Cabanillas-Gonzalez, G. Grancini, and G. Lanzani, "Pump-probe spectroscopy in organic semiconductors: monitoring fundamental processes of relevance in optoelectronics.," Advanced materials (Deerfield Beach, Fla.), vol. 23, pp. 5468-85, Dec. 2011.

[112] V. I. Klimov and D. W. McBranch, "Femtosecond high-sensitivity, chirp-free transient absorption spectroscopy using kilohertz lasers.," Optics letters, vol. 23, pp. 277-9, Feb. 1998.

[113] U. Megerle, I. Pugliesi, C. Schriever, C. F. Sailer, and E. Riedle, "Sub-50 fs broadband absorption spectroscopy with tunable excitation: putting the analysis of ultrafast molecular dynamics onsolid ground," Applied Physics B, vol. 96, pp. 215-231, June 2009.

[114] S. Westenhoff, I. a. Howard, J. M. Hodgkiss, K. R. Kirov, H. a. Bronstein, C. K. Williams, N. C. Greenham, and R. H. Friend, "Charge recombination in organic photovoltaic devices with high open-circuit voltages.," Journal of the American Chemical Society, vol. 130, pp. 13653-8, Oct. 2008.

[115] J. M. Hodgkiss, A. R. Campbell, R. A. Marsh, A. Rao, S. Albert-Seifried, and R. H. Friend, "Subnanosecond Geminate Charge Recombination in Polymer-Polymer Photovoltaic Devices," Physical Review Letters, vol. 104, p. 177701, Apr. 2010.

[116] C. Manzoni, D. Polli, and G. Cerullo, "Two-color pump-probe system broadly tunable over the visible and the near infrared with sub-30fs temporal resolution," Review of Scientific Instruments, vol. 77, no. 2, p. 023103, 2006. 
[117] M. Lorenc, M. Ziolek, R. Naskrecki, J. Karolczak, J. Kubicki, and a. Maciejewski, “Artifacts in femtosecond transient absorption spectroscopy," Applied Physics B: Lasers and Optics, vol. 74, pp. 19-27, Jan. 2002.

[118] W. Lee, H. Choi, S. Hwang, J. Y. Kim, and H. Y. Woo, "Efficient conventional- and invertedtype photovoltaic cells using a planar alternating polythiophene copolymer.," Chemistry (Weinheim an der Bergstrasse, Germany), vol. 18, pp. 2551-8, Feb. 2012.

[119] S. Gélinas, J. Kirkpatrick, I. a. Howard, K. Johnson, M. W. B. Wilson, G. Pace, R. H. Friend, and C. Silva, "Recombination Dynamics of Charge Pairs in a Push-Pull Polyfluorene-Derivative.," The journal of physical chemistry. B, Nov. 2012.

[120] J. Clark, C. Silva, R. H. Friend, and F. Spano, "Role of Intermolecular Coupling in the Photophysics of Disordered Organic Semiconductors: Aggregate Emission in Regioregular Polythiophene," Physical Review Letters, vol. 98, pp. 1-4, May 2007.

[121] F. C. Spano and C. Silva, "H- and J-aggregate behavior in polymeric semiconductors.," Annual review of physical chemistry, vol. 65, pp. 477-500, Jan. 2014.

[122] C. Soci, I.-W. Hwang, D. Moses, Z. Zhu, D. Waller, R. Gaudiana, C. Brabec, and a.J. Heeger, "Photoconductivity of a Low-Bandgap Conjugated Polymer," Advanced Functional Materials, vol. 17, pp. 632-636, Mar. 2007.

[123] C.-X. Sheng, M. Tong, S. Singh, and Z. Vardeny, "Experimental determination of the charge/neutral branching ratio $\eta$ in the photoexcitation of $\pi$-conjugated polymers by broadband ultrafast spectroscopy," Physical Review B, vol. 75, p. 085206, Feb. 2007.

[124] P. D. Cunningham and L. M. Hayden, "Carrier Dynamics Resulting from Above and Below Gap Excitation of P3HT and P3HT/PCBM Investigated by Optical-Pump Terahertz-Probe Spectroscopy ," The Journal of Physical Chemistry C, vol. 112, pp. 7928-7935, May 2008.

[125] F. Paquin, G. Latini, M. Sakowicz, P.-L. Karsenti, L. Wang, D. Beljonne, N. Stingelin, and C. Silva, "Charge Separation in Semicrystalline Polymeric Semiconductors by Photoexcitation: Is the Mechanism Intrinsic or Extrinsic?," Physical Review Letters, vol. 106, p. 197401, May 2011.

[126] O. G. Reid, R. D. Pensack, Y. Song, G. D. Scholes, and G. Rumbles, “Charge Photogeneration in Neat Conjugated Polymers," Chemistry of Materials, Oct. 2013.

[127] C. Schriever, S. Lochbrunner, E. Riedle, and D. J. Nesbitt, “Ultrasensitive ultraviolet-visible $20 \mathrm{fs}$ absorption spectroscopy of low vapor pressure molecules in the gas phase.," The Review of scientific instruments, vol. 79, p. 013107, Jan. 2008.

[128] G. Grancini, D. Polli, D. Fazzi, J. Cabanillas-Gonzalez, G. Cerullo, and G. Lanzani, "Transient Absorption Imaging of P3HT:PCBM Photovoltaic Blend: Evidence For Interfacial Charge Transfer State," The Journal of Physical Chemistry Letters, vol. 2, pp. 1099-1105, May 2011.

[129] S. S. Lo, M. S. Devadas, T. a. Major, and G. V. Hartland, “Optical detection of single nanoobjects by transient absorption microscopy.," The Analyst, vol. 138, pp. 25-31, Jan. 2013. 
[130] J. J. van Thor, "Photoreactions and dynamics of the green fluorescent protein.," Chemical Society reviews, vol. 38, pp. 2935-50, Oct. 2009.

[131] T. E. Villafana, W. P. Brown, J. K. Delaney, M. Palmer, W. S. Warren, and M. C. Fischer, "Femtosecond pump-probe microscopy generates virtual cross-sections in historic artwork.," Proceedings of the National Academy of Sciences of the United States of America, vol. 111, pp. 170813, Feb. 2014.

[132] J. Müller, J. Lupton, J. Feldmann, U. Lemmer, M. Scharber, N. Sariciftci, C. Brabec, and $\mathrm{U}$. Scherf, "Ultrafast dynamics of charge carrier photogeneration and geminate recombination in conjugated polymer:fullerene solar cells," Physical Review B, vol. 72, p. 195208, Nov. 2005.

[133] E. Busby, E. C. Carroll, E. M. Chinn, L. Chang, A. J. Moulé, and D. S. Larsen, “Excited-State Self-Trapping and Ground-State Relaxation Dynamics in Poly(3-hexylthiophene) Resolved with Broadband PumpDumpProbe Spectroscopy," The Journal of Physical Chemistry Letters, vol. 2, pp. 2764-2769, Nov. 2011.

[134] M. Tomasulo, S. Sortino, and F. M. Raymo, "A fast and stable photochromic switch based on the opening and closing of an oxazine ring.," Organic letters, vol. 7, pp. 1109-12, Mar. 2005.

[135] M. Tomasulo, S. Sortino, A. J. P. White, and F. M. Raymo, "Fast and stable photochromic oxazines.," The Journal of organic chemistry, vol. 70, pp. 8180-9, Sept. 2005.

[136] D. Polli, D. Brida, S. Mukamel, G. Lanzani, and G. Cerullo, "Effective temporal resolution in pump-probe spectroscopy with strongly chirped pulses," Physical Review A, vol. 82, p. 053809, Nov. 2010.

[137] B. Schmidt, S. Laimgruber, W. Zinth, and P. Gilch, "A broadband Kerr shutter for femtosecond fluorescence spectroscopy," Applied Physics B: Lasers and Optics, vol. 76, pp. 809-814, July 2003.

[138] R. Nakamura and Y. Kanematsu, "Femtosecond spectral snapshots based on electronic optical Kerr effect," Review of Scientific Instruments, vol. 75, no. 3, p. 636, 2004.

[139] A. Pivrikas, G. Juška, A. Mozer, M. Scharber, K. Arlauskas, N. Sariciftci, H. Stubb, and R. Österbacka, "Bimolecular Recombination Coefficient as a Sensitive Testing Parameter for Low-Mobility Solar-Cell Materials," Physical Review Letters, vol. 94, p. 176806, May 2005.

[140] A. Mozer, G. Dennler, N. Sariciftci, M. Westerling, A. Pivrikas, R. Österbacka, and G. Juška, "Time-dependent mobility and recombination of the photoinduced charge carriers in conjugated polymer/fullerene bulk heterojunction solar cells," Physical Review B, vol. 72, p. 035217, July 2005.

[141] C. G. Shuttle, B. ORegan, A. M. Ballantyne, J. Nelson, D. D. C. Bradley, J. de Mello, and J. R. Durrant, "Experimental determination of the rate law for charge carrier decay in a polythiophene: Fullerene solar cell," Applied Physics Letters, vol. 92, no. 9, p. 093311, 2008.

[142] C. G. Shuttle, B. ORegan, A. Ballantyne, J. Nelson, D. Bradley, and J. Durrant, "Bimolecular recombination losses in polythiophene: Fullerene solar cells," Physical Review B, vol. 78, p. 113201, Sept. 2008. 
[143] L. C. C. Elliott, J. I. Basham, K. P. Pernstich, P. R. Shrestha, L. J. Richter, D. M. DeLongchamp, and D. J. Gundlach, "Probing Charge Recombination Dynamics in Organic Photovoltaic Devices under Open-Circuit Conditions," Advanced Energy Materials, pp. n/a-n/a, June 2014.

[144] C. G. Shuttle, R. Hamilton, J. Nelson, B. C. O’Regan, and J. R. Durrant, "Measurement of Charge-Density Dependence of Carrier Mobility in an Organic Semiconductor Blend," Advanced Functional Materials, vol. 20, pp. 698-702, Mar. 2010.

[145] C. G. Shuttle, R. Hamilton, B. C. O’Regan, J. Nelson, and J. R. Durrant, “Charge-densitybased analysis of the current-voltage response of polythiophene/fullerene photovoltaic devices.," Proceedings of the National Academy of Sciences of the United States of America, vol. 107, pp. 16448-52, Sept. 2010.

[146] Y. Zhao, Z. Xie, Y. Qu, Y. Geng, and L. Wang, "Solvent-vapor treatment induced performance enhancement of poly(3-hexylthiophene):methanofullerene bulk-heterojunction photovoltaic cells," Applied Physics Letters, vol. 90, p. 043504, Jan. 2007.

[147] K. Takahashi, K. Seto, T. Yamaguchi, J.-i. Nakamura, C. Yokoe, and K. Murata, "Performance Enhancement by Blending an Electron Acceptor in TiO2/polyphenylenevinylene/Au Solidstate Solar Cells," Chemistry Letters, vol. 33, no. 8, pp. 1042-1043, 2004.

[148] S. H. Park, A. Roy, S. Beaupré, S. Cho, N. Coates, J. S. Moon, D. Moses, M. Leclerc, K. Lee, and A. J. Heeger, "Bulk heterojunction solar cells with internal quantum efficiency approaching 100\%," Nature Photonics, vol. 3, pp. 297-302, Apr. 2009.

[149] S. H. Park, A. Roy, S. Beaupré, S. Cho, N. Coates, J. S. Moon, D. Moses, M. Leclerc, K. Lee, and A. J. Heeger, "Bulk heterojunction solar cells with internal quantum efficiency approaching 100\%," Nature Photonics, vol. 3, pp. 297-302, Apr. 2009.

[150] N. Banerji, S. Cowan, M. Leclerc, E. Vauthey, and A. J. Heeger, "Exciton formation, relaxation, and decay in PCDTBT.," Journal of the American Chemical Society, vol. 132, pp. 17459-70, Dec. 2010.

[151] D. Jarzab, F. Cordella, J. Gao, M. Scharber, H.-J. Egelhaaf, and M. A. Loi, “Low-Temperature Behaviour of Charge Transfer Excitons in Narrow-Bandgap Polymer-Based Bulk Heterojunctions," Advanced Energy Materials, vol. 1, pp. 604-609, July 2011.

[152] D. D. Nuzzo, A. Aguirre, M. Shahid, V. S. Gevaerts, S. C. J. Meskers, and R. a. J. Janssen, "Improved film morphology reduces charge carrier recombination into the triplet excited state in a small bandgap polymer-fullerene photovoltaic cell.," Advanced materials (Deerfield Beach, Fla.), vol. 22, pp. 4321-4, Oct. 2010.

[153] M. Yan, L. Rothberg, E. Kwock, and T. Miller, “Interchain Excitations in Conjugated Polymers.," Physical review letters, vol. 75, pp. 1992-1995, Sept. 1995.

[154] K. Pichler, D. A. Halliday, D. D. C. Bradley, P. L. Burn, R. H. Friend, and A. B. Holmes, "Optical spectroscopy of highly ordered poly(p-phenylene vinylene)," Journal of Physics: Condensed Matter, vol. 5, pp. 7155-7172, Sept. 1993. 
[155] T. Offermans, P. van Hal, S. Meskers, M. Koetse, and R. Janssen, "Exciplex dynamics in a blend of $\pi$-conjugated polymers with electron donating and accepting properties: MDMOPPV and PCNEPV," Physical Review B, vol. 72, p. 045213, July 2005.

[156] G. F. Burkhard, E. T. Hoke, S. R. Scully, and M. D. McGehee, “Incomplete exciton harvesting from fullerenes in bulk heterojunction solar cells.," Nano letters, vol. 9, pp. 4037-41, Dec. 2009.

[157] F. Etzold, I. A. Howard, R. Mauer, M. Meister, T.-D. Kim, K.-S. Lee, N. S. Baek, and F. Laquai, "Ultrafast exciton dissociation followed by nongeminate charge recombination in PCDTBT:PCBM photovoltaic blends.," Journal of the American Chemical Society, vol. 133, pp. 9469-79, June 2011.

[158] T. Virgili, D. Marinotto, C. Manzoni, G. Cerullo, and G. Lanzani, "Ultrafast Intrachain Photoexcitation of Polymeric Semiconductors," Physical Review Letters, vol. 94, p. 117402, Mar. 2005.

[159] X. Yang, C.-L. Lee, S. Westenhoff, X. Zhang, and N. C. Greenham, "Saturation, Relaxation, and Dissociation of Excited Triplet Excitons in Conjugated Polymers," Advanced Materials, vol. 21, pp. 916-919, Feb. 2009.

[160] A. Maurano, R. Hamilton, C. G. Shuttle, A. M. Ballantyne, J. Nelson, B. O’Regan, W. Zhang, I. McCulloch, H. Azimi, M. Morana, C. J. Brabec, and J. R. Durrant, “Recombination dynamics as a key determinant of open circuit voltage in organic bulk heterojunction solar cells: a comparison of four different donor polymers.," Advanced Materials, vol. 22, pp. 4987-92, Nov. 2010.

[161] B. a. Gregg, S.-G. Chen, and R. a. Cormier, "Coulomb Forces and Doping in Organic Semiconductors," Chemistry of Materials, vol. 16, pp. 4586-4599, Nov. 2004.

[162] J. Guo, H. Ohkita, H. Benten, and S. Ito, "Charge generation and recombination dynamics in poly(3-hexylthiophene)/fullerene blend films with different regioregularities and morphologies.," Journal of the American Chemical Society, vol. 132, pp. 6154-64, May 2010.

[163] C. Yin, T. Kietzke, D. Neher, and H.-H. Horhold, "Photovoltaic properties and exciplex emission of polyphenylenevinylene-based blend solar cells," Applied Physics Letters, vol. 90, no. 9, p. 092117, 2007.

[164] Y. Zhou, K. Tvingstedt, F. Zhang, C. Du, W.-X. Ni, M. R. Andersson, and O. Inganäs, "Observation of a Charge Transfer State in Low-Bandgap Polymer/Fullerene Blend Systems by Photoluminescence and Electroluminescence Studies," Advanced Functional Materials, vol. 19, pp. 3293-3299, Oct. 2009.

[165] W. Ma, C. Yang, X. Gong, K. Lee, and a. J. Heeger, “Thermally Stable, Efficient Polymer Solar Cells with Nanoscale Control of the Interpenetrating Network Morphology," Advanced Functional Materials, vol. 15, pp. 1617-1622, Oct. 2005.

[166] A. R. Campbell, J. M. Hodgkiss, S. Westenhoff, I. A. Howard, R. A. Marsh, C. R. McNeill, R. H. Friend, and N. C. Greenham, "Low-temperature control of nanoscale morphology for high performance polymer photovoltaics.," Nano letters, vol. 8, pp. 3942-7, Nov. 2008. 
[167] D. Taguchi, T. Shino, L. Zhang, J. Li, M. Weis, T. Manaka, and M. Iwamoto, “Direct Probing of Photovoltaic Effect Generated in Double-Layer Organic Solar Cell by Electric-Field-Induced Optical Second-Harmonic Generation," Applied Physics Express, vol. 4, p. 021602, Jan. 2011.

[168] L. G. Kaake, a. Jailaubekov, K. J. Williams, and X.-Y. Zhu, "Probing ultrafast charge separation at organic donor/acceptor interfaces by a femtosecond electric field meter," Applied Physics Letters, vol. 99, no. 8, p. 083307, 2011.

[169] M. Meister, B. Baumeier, N. Pschirer, R. Sens, I. Bruder, F. Laquai, D. Andrienko, and I. A. Howard, "Observing Charge Dynamics in Surface Reactions by Time-Resolved Stark Effects," The Journal of Physical Chemistry C, vol. 117, pp. 9171-9177, May 2013.

[170] A. D. Chepelianskii, J. Wang, and R. H. Friend, "Low-Temperature Transport Properties of Photogenerated Charges in Organic Materials," Physical Review Letters, vol. 112, p. 126802, Mar. 2014.

[171] W. J. Grzegorczyk, T. J. Savenije, T. E. Dykstra, J. Piris, J. M. Schins, and L. D. Siebbeles, “Temperature-Independent Charge Carrier Photogeneration in P3HTPCBM Blends with Different Morphology," The Journal of Physical Chemistry C, vol. 114, pp. 5182-5186, Mar. 2010.

[172] J. Piris, T. E. Dykstra, A. a. Bakulin, P. H. V. Loosdrecht, W. Knulst, M. T. Trinh, J. M. Schins, and L. D. Siebbeles, "Photogeneration and Ultrafast Dynamics of Excitons and Charges in P3HT/PCBM Blends," The Journal of Physical Chemistry C, vol. 113, pp. 14500-14506, Aug. 2009.

[173] D. De Vault and B. Chance, "Studies of Photosynthesis Using a Pulsed Laser," Biophysical Journal, vol. 6, pp. 825-847, Nov. 1966.

[174] D. De Vault, J. H. Parkes, and B. Chance, "Electron Tunnelling in Cytochromes," Nature, vol. 215, pp. 642-644, Aug. 1967.

[175] J. Jortner, "Temperature dependent activation energy for electron transfer between biological molecules," The Journal of Chemical Physics, vol. 64, no. 12, p. 4860, 1976.

[176] J. J. Hopfield, “Electron Transfer Between Biological Molecules by Thermally Activated Tunneling," vol. 71, no. 9, pp. 3640-3644, 1974.

[177] T. Holstein, "Studies of polaron motion," Annals of Physics, vol. 8, pp. 343-389, Nov. 1959.

[178] P. F. Barbara, T. J. Meyer, and M. A. Ratner, "Contemporary Issues in Electron Transfer Research," The Journal of Physical Chemistry, vol. 100, pp. 13148-13168, Jan. 1996.

[179] H. B. Gray and J. R. Winkler, "Electron Flow through Proteins.," Chemical physics letters, vol. 483, pp. 1-9, Nov. 2009.

[180] B. Albinsson and J. Må rtensson, "Long-range electron and excitation energy transfer in donorbridgeacceptor systems," Journal of Photochemistry and Photobiology C: Photochemistry Reviews, vol. 9, pp. 138-155, Sept. 2008. 
[181] A. A. Paraecattil and N. Banerji, "Charge separation pathways in a highly efficient polymer: fullerene solar cell material.," Journal of the American Chemical Society, vol. 136, pp. 1472-82, Jan. 2014.

[182] H. M. McConnell, "Intramolecular Charge Transfer in Aromatic Free Radicals," The Journal of Chemical Physics, vol. 35, no. 2, p. 508, 1961.

[183] D. Beratan, J. Betts, and J. Onuchic, "Protein electron transfer rates set by the bridging secondary and tertiary structure," Science, vol. 252, pp. 1285-1288, May 1991.

[184] O. G. Reid and G. Rumbles, "Quantitative Transient Absorption Measurements of Polaron Yield and Absorption Coefficient in Neat Conjugated Polymers," The Journal of Physical Chemistry Letters, vol. 4, pp. 2348-2355, July 2013.

[185] R. J. O. M. Hoofman, M. P. D. Haas, L. D. A. Siebbeles, and J. M. Warman, “Highly mobile electrons and holes on isolated chains of the semiconducting polymer poly(phenylene vinylene)," Nature, vol. 392, no. March, pp. 54-56, 1998.

[186] M. Redecker and H. Bassler, "Optical detection of charge carriers in organic light-emitting diodes," Applied Physics Letters, vol. 69, no. 1, p. 70, 1996.

[187] J. Chappell, D. G. Lidzey, P. C. Jukes, A. M. Higgins, R. L. Thompson, S. O'Connor, I. Grizzi, R. Fletcher, J. O'Brien, M. Geoghegan, and R. a. L. Jones, “Correlating structure with fluorescence emission in phase-separated conjugated-polymer blends.," Nature materials, vol. 2, pp. 616-21, Sept. 2003.

[188] X. Zhang, Y. Xia, R. H. Friend, and C. Silva, "Sequential absorption processes in two-photonexcitation transient absorption spectroscopy in a semiconductor polymer," Physical Review B, vol. 73, p. 245201, June 2006.

[189] L. J. G. W. van Wilderen, C. N. Lincoln, and J. J. van Thor, “Modelling multi-pulse population dynamics from ultrafast spectroscopy.," PloS one, vol. 6, p. e17373, Jan. 2011.

[190] A. Morteani, A. Dhoot, J.-S. Kim, C. Silva, N. Greenham, C. Murphy, E. Moons, S. Ciná, J. Burroughes, and R. Friend, "Barrier-Free ElectronHole Capture in Polymer Blend Heterojunction Light-Emitting Diodes," Advanced Materials, vol. 15, pp. 1708-1712, Oct. 2003.

[191] J. R. Lakowicz, Principles of fluorescence spectroscopy. 2006.

[192] M. Albota, “Design of Organic Molecules with Large Two-Photon Absorption Cross Sections," Science, vol. 281, pp. 1653-1656, Sept. 1998.

[193] P. C. Tapping and T. W. Kee, "Optical Pumping of Poly(3-hexylthiophene) Singlet Excitons Induces Charge Carrier Generation," The Journal of Physical Chemistry Letters, vol. 5, pp. 10401047, Mar. 2014. 